Supporting Information

\title{
Hydroarylation of Alkenes Using Anilines in HFIP
}

Ignacio Colomer*[a], [b] 
Table of Contents

1. General experimental details $\quad$ S3

2. Optimization details $\quad$ S3

3. General procedure for the hydroarylation of alkenes using anilines in HFIP S5

4. Mechanistic studies $\quad$ S27

5. References $\quad$ S38

6. NMR spectra $\quad$ S39 


\section{General experimental details}

${ }^{1} \mathrm{H}$ NMR and ${ }^{13} \mathrm{C}$ NMR spectra were recorded on a $300 \mathrm{MHz}$ or $400 \mathrm{MHz}$ spectrometer in $\mathrm{CDCl}_{3}$ or $\mathrm{CD}_{3} \mathrm{OD}$ and referenced to residual solvent peaks. Chemical shifts are quoted in ppm (parts per million) to the nearest $0.01 \mathrm{ppm}$ with signal splitting recorded as singlet (s), doublet (d), triplet (t), quartet (q), quintet (quint), sextet (sext), septet (sept), multiplet (m) and broad singlet (br. s). Coupling constants, $J$, are measured in $\mathrm{Hz}$ to the nearest $0.1 \mathrm{~Hz} .{ }^{1} \mathrm{H}$ and ${ }^{13} \mathrm{C}$ NMR spectra were recorded at room temperature. High resolution mass spectra are given to four decimal places and were registered in a spectrometer GCT Agilent Technologies 6890N using Electronic Impact (E.I.) techniques at $70 \mathrm{eV}$, Fast Atom Bombardment and electrospray (ESI ${ }^{+}$or ESI-). Melting points (m.p.) were obtained from recrystallized samples using a Lecia VMTG heated-stage microscope and are uncorrected. The solvent systems used for recrystallization are quoted in parentheses. Flash column chromatography was performed using silica gel (60 A, 0.033-0.070 mm, BDH). TLC analyses were performed on Merck Kiesegel $60 \mathrm{~F}_{254} 0.25 \mathrm{~mm}$ precoated silica plates. Reagents obtained from Sigma-Aldrich, Alfa, Fluorochem and TCI suppliers were used directly as supplied. All anhydrous reactions were carried out in flame dried glassware and under an inert atmosphere of argon provided by a balloon. All reactions were stirred with magnetic followers. THF, ACN, toluene and $\mathrm{CH}_{2} \mathrm{Cl}_{2}$ were dried by purification through two activated alumina purification columns.

Alkenes $\mathbf{2 a}, \mathbf{2} \mathbf{g}$ and $\mathbf{2} \mathbf{i}$ are commercially available and were used without further purification. Alkenes $\mathbf{2 b},{ }^{1} \mathbf{2} \mathbf{c},{ }^{1} \mathbf{2 d},{ }^{1} \mathbf{2} \mathbf{e},{ }^{2} \mathbf{2} \mathbf{f}^{3}$ and $\mathbf{2} \mathbf{h}^{3}$ were synthesized following well stablished Wittig olefination procedures described in the literature.

\section{Optimization details.}

\subsection{Influence of the Temperature}

Keeping constant the 1:1 stoichiometry of aniline:alkene the effect of the temperature was studied.

Table S1: Optimisation of the hydroarylation: effect of the temperature ${ }^{[a]}$

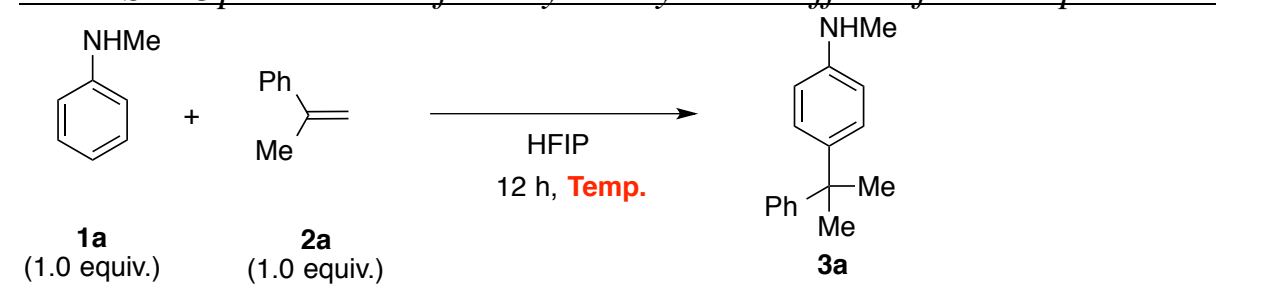

\begin{tabular}{c|c|c|c|c|c} 
Entry & $\begin{array}{c}\text { Temperature } \\
\left({ }^{\circ} \mathbf{C}\right)\end{array}$ & $\begin{array}{c}\text { Conversion } \\
(\boldsymbol{\%})\end{array}$ & $\begin{array}{c}\text { 1a recovered } \\
(\boldsymbol{\%})^{[b]}\end{array}$ & $\begin{array}{c}\text { 3a yield } \\
(\boldsymbol{\%})^{[b]}\end{array}$ & $\begin{array}{c}\text { 3a yield (\%), } \\
\text { based on 1a recovered }\end{array}$ \\
\hline 1 & $\mathbf{2 5}$ & 17 & 83 & 14 & 82 \\
\hline 2 & $\mathbf{4 0}$ & 27 & 73 & 19 & 70 \\
\hline 3 & $\mathbf{6 0}$ & 41 & 59 & 25 & 61 \\
\hline 4 & $\mathbf{8 0}$ & 47 & 53 & $\mathbf{2 9}$ & $\mathbf{6 2}$
\end{tabular}

[a] all reactions run on a $0.2 \mathrm{mmol}$ scale. [b] NMR yield using 1,3,5-trimethoxybenzene as standard. 


\subsection{Influence of alkene stoichiometry}

Keeping constant the temperature $\left(80^{\circ} \mathrm{C}\right)$ the effect of aniline:alkene stoichiometry was studied. Table S2: Optimisation of the hydroarylation: effect of the alkene stoichiometry. ${ }^{[a]}$

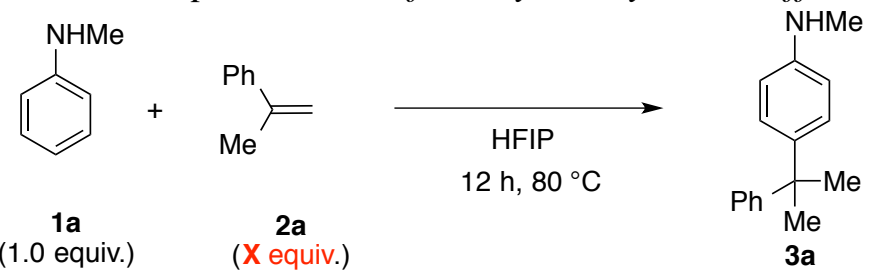

\begin{tabular}{c|c|c|c|c|c} 
Entry & 2a equivalent & $\begin{array}{c}\text { Conversion } \\
(\%)\end{array}$ & $\begin{array}{c}\text { 1a recovered } \\
(\boldsymbol{\%})^{[b]}\end{array}$ & $\begin{array}{c}\text { 3a yield } \\
(\%)^{[b]}\end{array}$ & $\begin{array}{c}\text { 3a yield (\%), } \\
\text { based on 1a recovered }\end{array}$ \\
\hline 1 & $\mathbf{1 . 0}$ & 47 & 53 & 29 & 62 \\
\hline 2 & $\mathbf{1 . 5}$ & 59 & 41 & 37 & 63 \\
\hline 3 & $\mathbf{2 . 0}$ & 61 & 39 & $\mathbf{4 1}$ & $\mathbf{6 4}$ \\
\hline 4 & $\mathbf{3 . 0}$ & 70 & 30 & 42 & 60
\end{tabular}

[a] all reactions run on a $0.2 \mathrm{mmol}$ scale. [b] NMR yield using 1,3,5-trimethoxybenzene as standard.

\subsection{Influence of additives}

Keeping constant the temperature $\left(80^{\circ} \mathrm{C}\right)$ and the $1: 2$ aniline:alkene stoichiometry the effect of additives was studied.

Table S3: Optimisation of the hydroarylation: effect of additives ${ }^{\left[{ }^{[a]}\right.}$

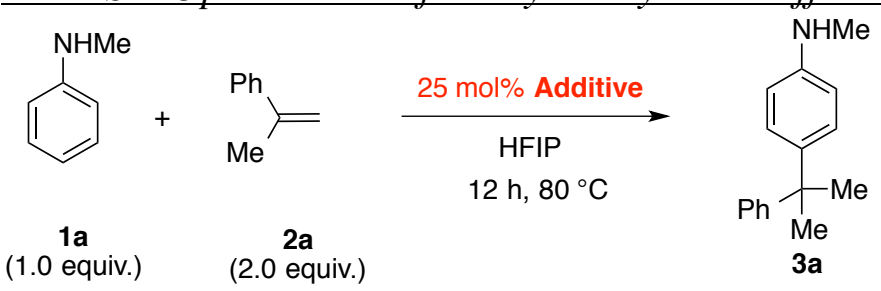

\begin{tabular}{c|c|c|c|c|c} 
Entry & Additive & $\begin{array}{c}\text { Conversion } \\
(\boldsymbol{\%})\end{array}$ & $\begin{array}{c}\text { 1a recovered } \\
(\boldsymbol{\%})^{[b]}\end{array}$ & $\begin{array}{c}\text { 3a yield } \\
(\boldsymbol{\%})^{[b]}\end{array}$ & $\begin{array}{c}\text { 3a yield (\%), } \\
\text { based on 1a recovered }\end{array}$ \\
\hline 1 & LiOAc & 89 & 11 & 44 & 49 \\
\hline 2 & NaOAc & 95 & 5 & $\mathbf{8 1}$ & $\mathbf{8 5}$ \\
\hline 3 & KOAc & 100 & 0 & 10 & 10 \\
\hline 4 & $\mathbf{A g O A c}$ & 100 & 0 & 43 & 43 \\
\hline 5 & $\mathbf{P d ( O A c )})_{2}$ & 100 & 0 & 18 & 18 \\
\hline 6 & $\mathbf{C u O A c}$ & 100 & 0 & 0 & 0 \\
\hline 7 & $\mathbf{C u}(\mathbf{O A c})_{2}$ & 100 & 0 & 40 & 40 \\
\hline 8 & $\mathbf{C C l}_{3} \mathbf{C O}_{2} \mathbf{N a}$ & 85 & 15 & 63 & 61 \\
\hline 9 & $\boldsymbol{t}$-BuOAc & 70 & 30 & 43 & 65
\end{tabular}

[a] all reactions run on a $0.2 \mathrm{mmol}$ scale. [b] NMR yield using 1,3,5-trimethoxybenzene as standard. 


\subsection{Influence of the additive stoichiometry}

Keeping constant the temperature $\left(80^{\circ} \mathrm{C}\right)$, the $1: 2$ aniline:alkene stoichiometry and $\mathrm{NaOAc}$ as additive, the effect of $\mathrm{NaOAc}$ stoichiometry was studied.

Table S4: Optimisation of the hydroarylation: effect of the additive stoichiometry. ${ }^{[a]}$
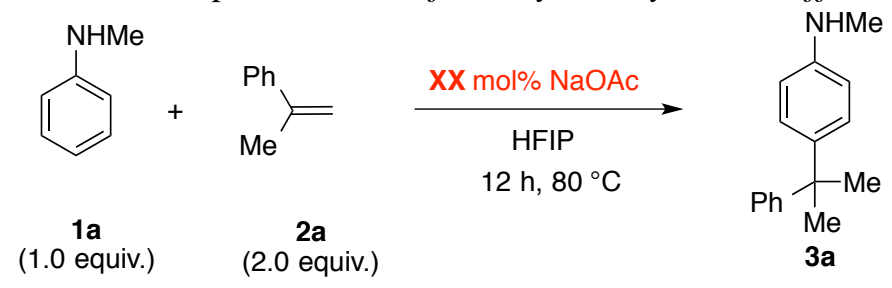

\begin{tabular}{c|c|c|c|c|c} 
Entry & $\begin{array}{c}\text { NaOAc } \\
(\mathbf{m o l} \%)\end{array}$ & $\begin{array}{c}\text { Conversion } \\
(\boldsymbol{\%})\end{array}$ & $\begin{array}{c}\text { 1a recovered } \\
(\boldsymbol{\%})^{[b]}\end{array}$ & $\begin{array}{c}\text { 3a yield } \\
(\%)^{[b]}\end{array}$ & $\begin{array}{c}\text { 3a yield (\%), } \\
\text { based on 1a recovered }\end{array}$ \\
\hline 1 & $\mathbf{1 0}$ & 69 & 31 & 50 & 72 \\
\hline 2 & $\mathbf{2 5}$ & 95 & 5 & $\mathbf{8 1}$ & $\mathbf{8 5}$ \\
\hline 3 & $\mathbf{5 0}$ & 90 & 10 & 72 & 76 \\
\hline 4 & $\mathbf{7 5}$ & 95 & 5 & 72 & 70
\end{tabular}

[a] all reactions run on a $0.2 \mathrm{mmol}$ scale. [b] NMR yield using 1,3,5-trimethoxybenzene as standard.

\section{General procedure for the hydroarylation of alkenes using anilines in HFIP.}

To a $5 \mathrm{~mL}$ Biotage ${ }^{\circledR}$ microwave vial equipped with a stirrer bar was added the corresponding aniline, 1 (1.0 equiv.), the alkene, 2 (2.0 equiv.), NaOAc (25 - $100 \mathrm{~mol} \%)$ and HFIP (5.0 mL/mmol). The reaction vessel was sealed with a microwave vial cap (containing a Reseal ${ }^{\mathrm{TM}}$ septum) and purged with Ar. Following this, the vial was heated to the required temperature $\left(60{ }^{\circ} \mathrm{C}\right.$ or $\left.80{ }^{\circ} \mathrm{C}\right)$ in a preheated oil bath for $12 \mathrm{~h}$. The mixture was cooled to RT, monitored by TLC and the solvent was evaporated under reduced pressure. The crude reaction was purified by chromatography on silica gel using the appropriate mixture of eluents to give the corresponding product, $\mathbf{3}$.

\section{1. $N$-Methyl-4-(2-phenylpropan-2-yl)aniline, 3a.}

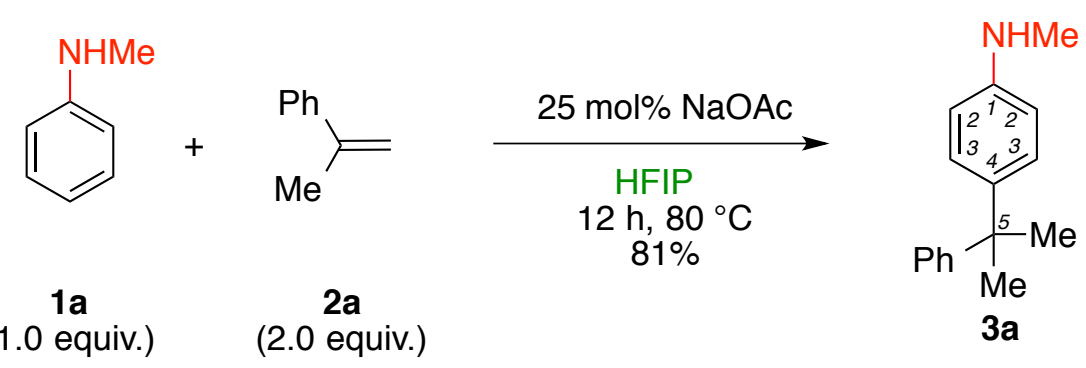

From aniline 1a (22 mg, $0.2 \mathrm{mmol})$, alkene 2a (52 $\mu \mathrm{l}, 0.4 \mathrm{mmol})$ and $\mathrm{NaOAc}(4.0 \mathrm{mg}, 0.05$ mmol), in $1.0 \mathrm{~mL}$ of HFIP, following the general procedure, aniline 3a was obtained. 
Chromatographic purification (gradient elution: 5:95 $\rightarrow$ 30:70 $\mathrm{Et}_{2} \mathrm{O}$ - hexane) gave 3a (37 mg, 81\%), as a yellow oil.

Data for 3a: $\boldsymbol{R}_{f} 0.35\left(25 \% \mathrm{Et}_{2} \mathrm{O}\right.$ - hexane). ${ }^{1} \mathbf{H}$ NMR (300 $\left.\mathbf{M H z}, \mathbf{C D C l}_{3}\right) \delta$ 7.15-7.31 (5 H, m, Ph), $7.08(2 \mathrm{H}, \mathrm{d}, J=8.6 \mathrm{~Hz}, 3-\mathrm{H}), 6.56(2 \mathrm{H}, \mathrm{d}, J=8.6 \mathrm{~Hz}, 2-\mathrm{H}), 3.61$ (1 H, br s, NH), 2.84 (3 H, s, NMe), 1.67 (6 H, s, 2 x Me). ${ }^{13}$ C NMR (75 MHz, CDCl $) \delta 151.5$ (1 C, C Ar), 147.2 (1 C, C Ar), 139.7 (1 C, C Ar), 128.0 (2 C, CH Ar), 127.7 (2 C, CH Ar), 126.9 (2 C, CH Ar), 125.5 (1 C, CH Ph), 112.2 (2 C, C-2), 42.3 (1 C, C-5), 31.03 (2 C, 2 x Me), 31.00 (1 C, NMe). HRMS (ESI): calculated for $\mathrm{C}_{16} \mathrm{H}_{20} \mathrm{~N}[\mathrm{M}+\mathrm{H}]^{+}$requires $m / z, 226.1590$, found $m / z, 226.1587$.

\section{2. $N$-Benzyl-4-(2-phenylpropan-2-yl)aniline, $3 \mathrm{~b}$.}

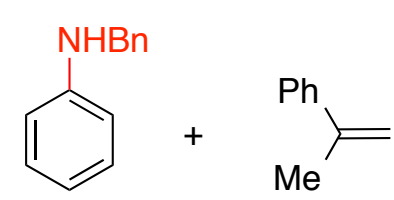

$1 \mathrm{~b}$ (1.0 equiv.)

2a

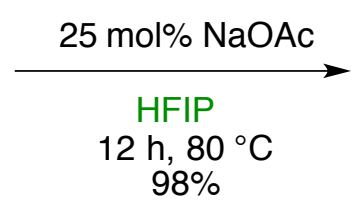
(2.0 equiv.)

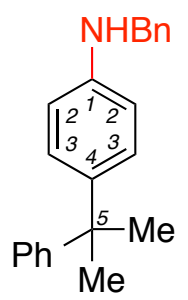

3b

From aniline $\mathbf{1 b}(28 \mathrm{mg}, 0.2 \mathrm{mmol})$, alkene $2 \mathbf{a}(52 \mu \mathrm{l}, 0.4 \mathrm{mmol})$ and $\mathrm{NaOAc}(4.0 \mathrm{mg}, 0.05$ mmol), in $1.0 \mathrm{~mL}$ of HFIP, following the general procedure, aniline $\mathbf{3 b}$ was obtained. Chromatographic purification (gradient elution: 5:95 $\rightarrow$ 30:70 $\mathrm{Et}_{2} \mathrm{O}$ - hexane) gave $\mathbf{3 b}(50 \mathrm{mg}$, $98 \%$ ), as a yellow oil.

Data for 3b: $\boldsymbol{R}_{f} 0.30\left(25 \% \mathrm{Et}_{2} \mathrm{O}\right.$ - hexane). ${ }^{1} \mathbf{H}$ NMR (300 $\left.\mathbf{M H z}, \mathbf{C D C l}_{3}\right) \delta 7.13-7.48(10 \mathrm{H}$, m, Ph), $7.06(2 \mathrm{H}, \mathrm{d}, J=8.7 \mathrm{~Hz}, 3-\mathrm{H}), 6.58(2 \mathrm{H}, \mathrm{d}, J=8.7 \mathrm{~Hz}, 2-\mathrm{H}), 4.31\left(2 \mathrm{H}, \mathrm{s}, \mathrm{CH}_{2} \mathrm{Bn}\right), 3.93(1$ H, br s, NH), 1.66 (6 H, s, 2 x Me). $\left.{ }^{13} \mathbf{C ~ N M R ~ ( 7 5 ~ M H z , ~ C D C l ~}\right) \delta 151.4$ (1 C, C Ar), 146.1 (1 C, C Ar), 140.0 (1 C, C Ar), 139.8 (1 C, C Ar), 128.8 (2 C, CH Ar), 128.0 (2 C, CH Ar), 127.76 (2 C, CH Ar), 127.75 (2 C, CH Ar), 127.4 (1 C, CH Ar), 126.9 (2 C, CH Ar), 125.5 (1 C, CH Ar), 112.6 (2 C, C-2), 48.8 (1 C, $\left.\mathrm{CH}_{2} \mathrm{Bn}\right), 42.3$ (1 C, C-5), 31.0 (2 C, 2 x Me). HRMS (ESI): calculated for $\mathrm{C}_{22} \mathrm{H}_{24} \mathrm{~N}$ $[\mathrm{M}+\mathrm{H}]^{+}$requires $m / z$ 302.1903, found $m / z$ 302.1912.

\section{3. $\quad N$-Allyl-4-(2-phenylpropan-2-yl)aniline, 3c.}

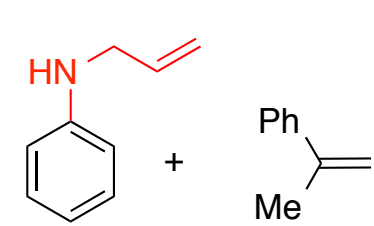

1c (1.0 equiv.)

$2 a$

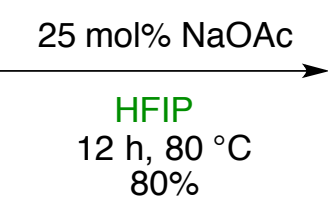
(2.0 equiv.)

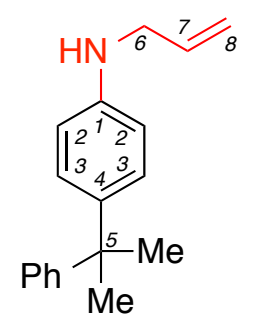

3c 
From aniline 1c $(27 \mathrm{mg}, 0.2 \mathrm{mmol})$, alkene $2 \mathbf{a}(52 \mu \mathrm{l}, 0.4 \mathrm{mmol})$ and $\mathrm{NaOAc}(4.0 \mathrm{mg}, 0.05$ $\mathrm{mmol}$ ), in $1.0 \mathrm{~mL}$ of HFIP, following the general procedure, aniline $3 \mathbf{c}$ was obtained. Chromatographic purification (gradient elution: 5:95 $\rightarrow$ 30:70 $\mathrm{Et}_{2} \mathrm{O}$ - hexane) gave $3 \mathbf{c}(40 \mathrm{mg}, 80 \%)$, as a yellow oil.

Data for 3c: $\boldsymbol{R}_{f} 0.30\left(25 \% \mathrm{Et}_{2} \mathrm{O}\right.$ - hexane). ${ }^{1} \mathbf{H}$ NMR (300 $\left.\mathbf{M H z}, \mathbf{C D C l}_{3}\right) \delta$ 7.14-7.31 (5 H, m, $\mathrm{Ph}), 7.06(2 \mathrm{H}, \mathrm{d}, J=8.6 \mathrm{~Hz}, 3-\mathrm{H}), 6.57(2 \mathrm{H}, \mathrm{d}, J=8.6 \mathrm{~Hz}, 2-\mathrm{H}), 5.98(1 \mathrm{H}, \mathrm{ddt}, J=17.2,10.3$ and $5.5 \mathrm{~Hz}, 7-\mathrm{H}), 5.30\left(1 \mathrm{H}, \mathrm{dq}, J=17.2\right.$ and $\left.1.6 \mathrm{~Hz}, 8-\mathrm{H}_{\text {trans }}\right), 5.18\left(1 \mathrm{H}, \mathrm{dq}, J=10.3\right.$ and $\left.1.5 \mathrm{~Hz}, 8-\mathrm{H}_{\text {cis }}\right)$, $3.77\left(2 \mathrm{H}, \mathrm{dt}, J=5.5\right.$ and $\left.1.6 \mathrm{~Hz}, 6-\mathrm{H}_{2}\right), 3.69(1 \mathrm{H}, \mathrm{s}, \mathrm{NH}), 1.66(6 \mathrm{H}, \mathrm{s}, 2 \mathrm{x} \mathrm{Me}) .{ }^{13} \mathrm{C} \mathrm{NMR}(75 \mathrm{MHz}$, $\left.\mathbf{C D C l}_{3}\right) \delta 151.5$ (1 C, C Ar), 146.0 (1 C, C Ar), 140.0 (1 C, C Ar), 135.9 (1 C, C-7), 128.0 (2 C, CH Ar), 127.7 (2 C, CH Ar), 126.9 (2 C, CH Ar), 125.5 (1 C, CH Ph), 116.3 (1 C, C-8), 112.7 (2 C, C2), 47.0 (1 C, C-6), 42.3 (1 C, C-5), 31.0 (2 C, NMe 2 ), 31.0 (2 C, 2 x Me). HRMS (ESI): calculated for $\mathrm{C}_{18} \mathrm{H}_{22} \mathrm{~N}[\mathrm{M}+\mathrm{H}]^{+}$requires $m / z, 252.1746$, found $m / z, 252.1754$.

\subsection{2-\{ [4-(2-phenylpropan-2-yl)phenyl]amino\}ethan-1-ol, $3 d$.}<smiles>C=C(C)Pc1ccccc1</smiles>

1d (1.0 equiv.)

$2 a$

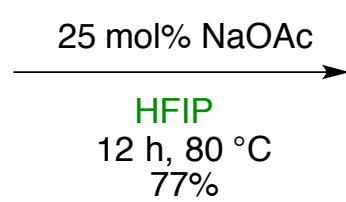
(2.0 equiv.)

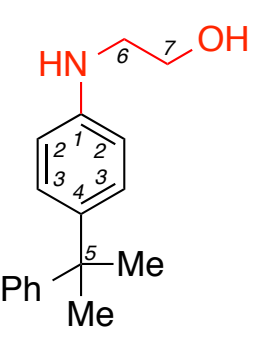

3d

From aniline 1d (28 mg, $0.2 \mathrm{mmol})$, alkene $2 \mathbf{a}(52 \mu \mathrm{l}, 0.4 \mathrm{mmol})$ and $\mathrm{NaOAc}(4.0 \mathrm{mg}, 0.05$ mmol), in $1.0 \mathrm{~mL}$ of HFIP, following the general procedure, aniline $\mathbf{3 d}$ was obtained. Chromatographic purification (gradient elution: 5:95 $\rightarrow$ 30:70 $\mathrm{Et}_{2} \mathrm{O}$ - hexane) gave $3 \mathbf{d}(39 \mathrm{mg}, 77 \%$ ), as a yellow oil.

Data for 3d: $\boldsymbol{R}_{f} 0.25\left(50 \% \mathrm{Et}_{2} \mathrm{O}\right.$ - hexane). ${ }^{1} \mathbf{H}$ NMR (300 $\left.\mathbf{M H z}, \mathbf{C D C l}_{3}\right) \delta$ 7.15-7.35 (5 H, m, Ph), $7.08(2 \mathrm{H}, \mathrm{d}, J=8.7 \mathrm{~Hz}, 3-\mathrm{H}), 6.61(2 \mathrm{H}, \mathrm{d}, J=8.7 \mathrm{~Hz}, 2-\mathrm{H}), 3.84\left(2 \mathrm{H}, \mathrm{t}, J=5.2 \mathrm{~Hz}, 7-\mathrm{H}_{2}\right), 3.32$ $\left(2 \mathrm{H}, \mathrm{t}, J=5.2 \mathrm{~Hz}, 6-\mathrm{H}_{2}\right), 2.54(2 \mathrm{H}, \mathrm{br} \mathrm{s}, \mathrm{OH}$ and $\mathrm{NH}), 1.66(6 \mathrm{H}, \mathrm{s}, 2 \mathrm{x} \mathrm{Me}) .{ }^{13} \mathbf{C}$ NMR (75 MHz, $\left.\mathbf{C D C l}_{3}\right) \delta 151.4$ (1 C, C Ar), 145.9 (1 C, C Ar), 140.6 (1 C, C Ar), 128.0 (2 C, CH Ar), 127.8 (2 C, CH Ar), 126.9 (2 C, CH Ar), 125.6 (1 C, CH Ph), 113.1 (2 C, C-2), 61.5 (1 C, C-7), 46.5 (1 C, C-6), 42.3 (1 C, C-5), 31.0 (2 C, 2 x Me). HRMS (ESI): calculated for $\mathrm{C}_{17} \mathrm{H}_{22} \mathrm{NO}[\mathrm{M}+\mathrm{H}]^{+}$requires $\mathrm{m} / z$ 256.1695 , found $m / z, 256.1694$. 
3.5. 4-\{[4-(2-Phenylpropan-2-yl)phenyl $]$ amino $\}$ phenol, 3 e.<smiles>C=C(C)Pc1ccccc1</smiles>

1e (1.0 equiv.)

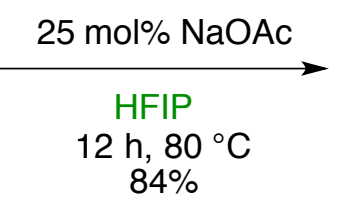

$2 a$

(2.0 equiv.)

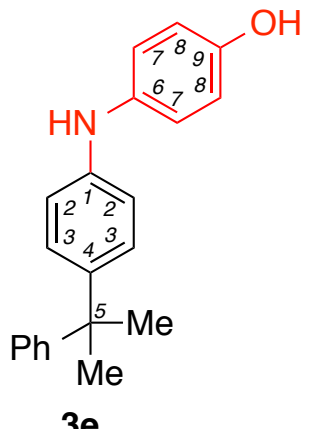

$3 e$

From aniline 1e (37 mg, $0.2 \mathrm{mmol})$, alkene $2 \mathbf{a}(52 \mu \mathrm{l}, 0.4 \mathrm{mmol})$ and $\mathrm{NaOAc}(4.0 \mathrm{mg}, 0.05$ $\mathrm{mmol}$ ), in $1.0 \mathrm{~mL}$ of HFIP, following the general procedure, aniline $\mathbf{3 e}$ was obtained. Chromatographic purification (gradient elution: 5:95 $\rightarrow$ 70:30 $\mathrm{Et}_{2} \mathrm{O}$ - hexane) gave 3e (51 mg, 84\%), as a red oil.

Data for 3e: $\boldsymbol{R}_{f} 0.30\left(50 \% \mathrm{Et}_{2} \mathrm{O}\right.$ - hexane). ${ }^{1} \mathbf{H}$ NMR (300 $\left.\mathbf{M H z}, \mathbf{C D C l}_{3}\right) \delta$ 7.16-7.29 (5 H, m, $\mathrm{Ph}), 7.09(2 \mathrm{H}, \mathrm{d}, J=8.7 \mathrm{~Hz}, 3-\mathrm{H}), 7.00(2 \mathrm{H}, \mathrm{d}, J=8.7 \mathrm{~Hz}, 8-\mathrm{H}), 6.84(2 \mathrm{H}, \mathrm{d}, J=8.7 \mathrm{~Hz}, 7-\mathrm{H}), 6.77$ $(2 \mathrm{H}, \mathrm{d}, J=8.7 \mathrm{~Hz}, 2-\mathrm{H}), 5.46(1 \mathrm{H}$, br s, OH $), 4.54\left(1 \mathrm{H}\right.$, br s, NH), $1.67(6 \mathrm{H}, \mathrm{s}, 2 \mathrm{x} \mathrm{Me}) .{ }^{13} \mathbf{C ~ N M R}$ (75 MHz, CDCl $\left.{ }_{3}\right) \delta 151.2$ (1 C, C Ar), 150.9 (1 C, C Ar), 142.7 (1 C, C Ar), 142.4 (1 C, C Ar), 136.4 (1 C, C Ar), 128.1 (2 C, CH Ar), 127.8 (2 C, CH Ar), 126.9 (2 C, CH Ar), 125.6 (1 C, CH Ph), 122.0 (2 C, C-8), 116.2 and 115.7 (4 C, C-2 and C-7), 42.5 (1 C, C-5), 31.0 (2 C, 2 x Me). HRMS (ESI): calculated for $\mathrm{C}_{21} \mathrm{H}_{22} \mathrm{NO}[\mathrm{M}+\mathrm{H}]^{+}$requires $m / z$ 304.1695, found $m / z$ 304.1697.

\section{6. $\quad N, N$-Dimethyl-4-(2-phenylpropan-2-yl)aniline, 3f.}<smiles>CN(C)c1ccccc1</smiles>

$1 f$<smiles>C=C(C)Pc1ccccc1</smiles>

$2 a$

(1.0 equiv.) (2.0 equiv.)

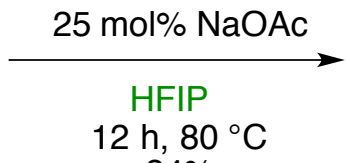

$84 \%$

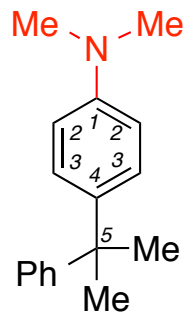

$3 f$

From aniline $1 \mathbf{f}(24 \mathrm{mg}, 0.2 \mathrm{mmol})$, alkene $\mathbf{2 a}(52 \mu 1,0.4 \mathrm{mmol})$ and $\mathrm{NaOAc}(4.0 \mathrm{mg}, 0.05$ $\mathrm{mmol}$ ), in $1.0 \mathrm{~mL}$ of HFIP, following the general procedure, aniline $\mathbf{3 f}$ was obtained. Chromatographic purification (gradient elution: 5:95 $\rightarrow 30: 70 \mathrm{Et}_{2} \mathrm{O}$ - hexane) gave $\mathbf{3 f}(40 \mathrm{mg}, 84 \%$ ), as a yellow oil.

Data for 3f: $\boldsymbol{R}_{f} 0.40\left(25 \% \mathrm{Et}_{2} \mathrm{O}\right.$ - hexane). ${ }^{1} \mathbf{H}$ NMR (300 $\left.\mathbf{M H z}, \mathbf{C D C l}_{3}\right) \delta$ 7.15-7.31 (5 H, m, $\mathrm{Ph}), 7.12(2 \mathrm{H}, \mathrm{d}, J=8.8 \mathrm{~Hz}, 3-\mathrm{H}), 6.68(2 \mathrm{H}, \mathrm{d}, J=8.7 \mathrm{~Hz}, 2-\mathrm{H}), 2.92\left(6 \mathrm{H}, \mathrm{s}, \mathrm{NMe}_{2}\right), 1.68(6 \mathrm{H}, \mathrm{s}$, 2 x Me). ${ }^{13} \mathbf{C}$ NMR (75 MHz, CDCl $) \delta 151.5$ (1 C, C Ar), 148.7 (1 C, C Ar), 139.9 (1 C, C Ar), 128.0 (2 C, $\mathrm{CH} \mathrm{Ar}), 127.6$ (2 C, CH Ar), 126.9 (2 C, CH Ar), 125.5 (1 C, CH Ph), 112.5 (2 C, C-2), 
42.2 (1 C, C-5), 31.0 (2 C, $\mathrm{NMe}_{2}$ ), 31.0 (2 C, 2 x Me). HRMS (EI): calculated for $\mathrm{C}_{17} \mathrm{H}_{21} \mathrm{~N}$ [M] ${ }^{+}$ requires $m / z, 239.1674$, found $m / z 239.1676$.

\subsection{1-[4-(2-Phenylpropan-2-yl)phenyl]piperazine, 3g.}<smiles>C=C(C)c1ccccc1</smiles>

(1.0 equiv.)

(2.0 equiv.)

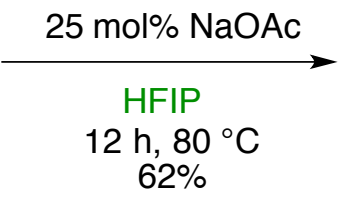

$62 \%$

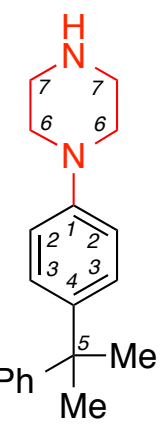

$3 g$

From aniline $1 \mathbf{g}$ (32 mg, $0.2 \mathrm{mmol})$, alkene $2 \mathbf{a}(52 \mu \mathrm{l}, 0.4 \mathrm{mmol})$ and $\mathrm{NaOAc}(4.0 \mathrm{mg}, 0.05$ $\mathrm{mmol}$ ), in $1.0 \mathrm{~mL}$ of HFIP, following the general procedure, aniline $\mathbf{3 g}$ was obtained. Chromatographic purification (gradient elution: $1: 99 \rightarrow 20: 80 \mathrm{MeOH}-\mathrm{CH}_{2} \mathrm{Cl}_{2}$ ) gave $3 \mathrm{~g}(35 \mathrm{mg}$, $62 \%$ ), as a yellow foam.

Data for 3g: $\boldsymbol{R}_{f} 0.20\left(5 \% \mathrm{MeOH}-\mathrm{CH}_{2} \mathrm{Cl}_{2}\right) .{ }^{1} \mathbf{H}$ NMR (300 MHz, $\mathbf{C D}_{3}$ OD) $\delta$ 7.16-7.30 (5 H, $\mathrm{m}, \mathrm{Ph}), 7.11(2 \mathrm{H}, \mathrm{d}, J=8.9 \mathrm{~Hz}, 3-\mathrm{H}), 6.88(2 \mathrm{H}, \mathrm{d}, J=8.9 \mathrm{~Hz}, 2-\mathrm{H}), 3.12(4 \mathrm{H}, \mathrm{dd}, J=6.4$ and 3.4 $\left.\mathrm{Hz}, 6-\mathrm{H}_{2}\right), 3.01\left(4 \mathrm{H}, \mathrm{dd}, J=6.4\right.$ and $\left.3.4 \mathrm{~Hz}, 7-\mathrm{H}_{2}\right), 1.63(6 \mathrm{H}, \mathrm{s}, 2 \mathrm{x} \mathrm{Me}) .{ }^{13} \mathrm{C}$ NMR (75 MHz, CD $_{3}$ OD) $\delta 152.3$ (1 C, C Ar), 150.7 (1 C, C Ar), 144.1 (1 C, C Ar), 128.9 (2 C, CH Ar), 128.5 (2 C, CH Ar), 127.8 (2 C, CH Ar), 126.5 (1 C, CH Ph), 117.4 (2 C, C-2), 51.0 and 46.3 (4 C, C-6 and C7), 43.2 (1 C, C-5), 31.3 (2 C, 2 x Me). HRMS (EI): calculated for $\mathrm{C}_{19} \mathrm{H}_{24} \mathrm{~N}_{2}[\mathrm{M}]^{+}$requires $\mathrm{m} / \mathrm{z}$ 280.1939 , found $\mathrm{m} / \mathrm{z} 280.1953$.

3.8. 4-(2-Phenylpropan-2-yl)aniline, $3 \mathrm{~h}$.<smiles>C=C(C)Pc1ccccc1</smiles>

$1 \mathrm{~h}$ (1.0 equiv.)

$2 a$

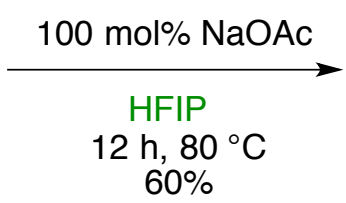
(2.0 equiv.)

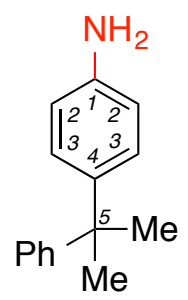

3h

From aniline 1h (19 mg, $0.2 \mathrm{mmol})$, alkene 2a (52 $\mu \mathrm{l}, 0.4 \mathrm{mmol})$ and $\mathrm{NaOAc}(16 \mathrm{mg}, 0.2$ $\mathrm{mmol}$ ), in $1.0 \mathrm{~mL}$ of HFIP, following the general procedure, aniline $\mathbf{3 h}$ was obtained. Chromatographic purification (gradient elution: 5:95 $\rightarrow$ 70:30 $\mathrm{Et}_{2} \mathrm{O}$ - hexane) gave $\mathbf{3 h}(25 \mathrm{mg}, 60 \%$ ), as a yellow oil.

Data for 3h: $\boldsymbol{R}_{f} 0.25\left(50 \% \mathrm{Et}_{2} \mathrm{O}-\right.$ hexane $) .{ }^{1} \mathbf{H} \mathbf{N M R}\left(\mathbf{3 0 0} \mathbf{M H z}, \mathbf{C D C l}_{3}\right) \delta 7.16-7.31(5 \mathrm{H}, \mathrm{m}$, 
$\mathrm{Ph}), 7.04(2 \mathrm{H}, \mathrm{d}, J=8.6 \mathrm{~Hz}, 3-\mathrm{H}), 6.62(2 \mathrm{H}, \mathrm{d}, J=8.6 \mathrm{~Hz}, 2-\mathrm{H}), 3.57\left(2 \mathrm{H}, \mathrm{br} \mathrm{s}, \mathrm{NH}_{2}\right), 1.66(6 \mathrm{H}, \mathrm{s}$, 2 x Me). ${ }^{13} \mathbf{C}$ NMR (75 MHz, CDCl $) \delta 151.4$ (1 C, C Ar), 144.1 (1 C, C Ar), 141.1 (1 C, C Ar), 128.0 (2 C, CH Ar), 127.8 (2 C, CH Ar), 126.9 (2 C, CH Ar), 125.6 (1 C, CH Ph), 115.0 (2 C, C-2), 42.3 (1 C, C-5), 31.0 (2 C, 2 x Me). HRMS (EI): calculated for $\mathrm{C}_{15} \mathrm{H}_{17} \mathrm{~N}[\mathrm{M}]^{+}$requires $\mathrm{m} / z$ 2 211.1361, found $m / z 211.1371$.

3.9. $\quad N, 2$-Dimethyl-4-(2-phenylpropan-2-yl)aniline, 3 i.<smiles>C=C(C)Pc1ccccc1</smiles>

$1 \mathbf{i}$ (1.0 equiv.) $\mathbf{2 a}$
$(2.0$ equiv. $)$

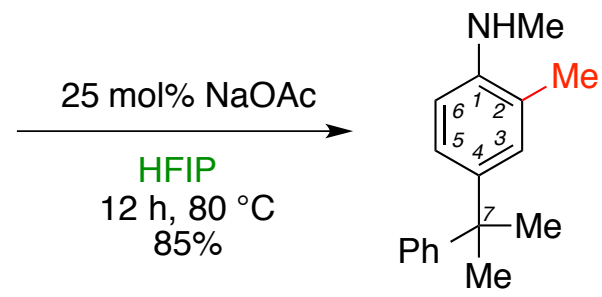

$3 \mathbf{i}$

From aniline 1i (24 mg, $0.2 \mathrm{mmol})$, alkene 2a (52 $\mu \mathrm{l}, 0.4 \mathrm{mmol})$ and $\mathrm{NaOAc}(4.0 \mathrm{mg}, 0.05$ mmol), in $1.0 \mathrm{~mL}$ of HFIP, following the general procedure, aniline $\mathbf{3 i}$ was obtained. Chromatographic purification (gradient elution: 5:95 $\rightarrow$ 30:70 $\mathrm{Et}_{2} \mathrm{O}-$ hexane) gave 3i (41 mg, 85\%), as a yellow oil.

Data for 3i: $\boldsymbol{R}_{f} 0.40\left(25 \% \mathrm{Et}_{2} \mathrm{O}\right.$ - hexane). ${ }^{1} \mathbf{H}$ NMR (300 $\left.\mathbf{M H z}, \mathbf{C D C l}_{3}\right) \delta 7.11-7.28(5 \mathrm{H}, \mathrm{m}$, $\mathrm{Ph}), 7.12(1 \mathrm{H}, \mathrm{dd}, J=8.3$ and $2.5 \mathrm{~Hz}, 5-\mathrm{H}), 6.91(1 \mathrm{H}, \mathrm{d}, J=2.4 \mathrm{~Hz}, 3-\mathrm{H}), 6.53(1 \mathrm{H}, \mathrm{d}, J=8.3 \mathrm{~Hz}$, 6-H), 3.43 (1 H, br s, NH), 2.86 (3 H, s, NMe), 2.08 (3 H, s, Me), 1.65 (6 H, s, 2 x Me). ${ }^{13}$ C NMR (75 MHz, $\left.\mathbf{C D C l}_{3}\right) \delta 151.6$ (1 C, C Ar), 145.2 (1 C, C Ar), 139.2 (1 C, C Ar), 128.9 (1 C, CH Ar), 128.0 (2 C, CH Ph), 126.9 (2 C, CH Ph), 125.4 (1 C, CH Ar), 125.3 (1 C, CH Ar), 121.7 (1 C, C Ar), 108.8 (1 C, C-6), 42.2 (1 C, C-7), 31.1 (1 C, NMe), 31.0 (2 C, 2 x Me), 17.8 (1 C, Me). HRMS (ESI): calculated for $\mathrm{C}_{17} \mathrm{H}_{22} \mathrm{~N}[\mathrm{M}+\mathrm{H}]^{+}$requires $m / z$ 240.1746, found $m / z, 240.1743$.

\subsection{2-Ethyl-4-(2-phenylpropan-2-yl)aniline, 3j.}<smiles>CCc1ccccc1N</smiles>

1j

(1.0 equiv.)<smiles>C=C(C)Pc1ccccc1</smiles>

$2 a$

(2.0 equiv.)

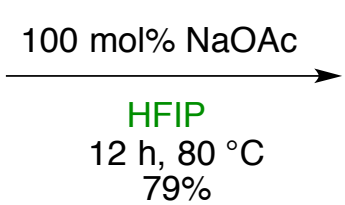

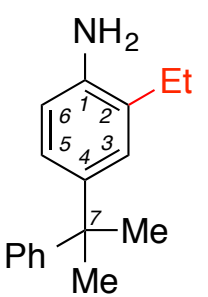

3j

From aniline 1j (24 mg, $0.2 \mathrm{mmol})$, alkene $2 \mathbf{a}(52 \mu \mathrm{l}, 0.4 \mathrm{mmol})$ and $\mathrm{NaOAc}(16 \mathrm{mg}, 0.2$ $\mathrm{mmol}$ ), in $1.0 \mathrm{~mL}$ of HFIP, following the general procedure, aniline $\mathbf{3 j}$ was obtained. 
Chromatographic purification (gradient elution: 5:95 $\rightarrow$ 70:30 $\mathrm{Et}_{2} \mathrm{O}$ - hexane) gave $\mathbf{3 j}$ (38 $\mathrm{mg}, 79 \%$ ), as a yellow oil.

Data for 3j: $\boldsymbol{R}_{f} 0.45\left(50 \% \mathrm{Et}_{2} \mathrm{O}\right.$ - hexane). ${ }^{1} \mathbf{H}$ NMR (300 $\left.\mathbf{M H z}, \mathbf{C D C l}_{3}\right) \delta$ 7.13-7.29 (5 H, m, $\mathrm{Ph}), 6.95(1 \mathrm{H}, \mathrm{d}, J=2.3 \mathrm{~Hz}, 3-\mathrm{H}), 6.89(1 \mathrm{H}, \mathrm{dd}, J=8.2$ and $2.3 \mathrm{~Hz}, 5-\mathrm{H}), 6.59(1 \mathrm{H}, \mathrm{d}, J=8.2 \mathrm{~Hz}$, 6-H), $3.51\left(2 \mathrm{H}\right.$, br s, $\left.\mathrm{NH}_{2}\right), 2.49\left(2 \mathrm{H}, \mathrm{q}, J=7.5 \mathrm{~Hz}, \mathrm{CH}_{2} \mathrm{Et}\right), 1.65(6 \mathrm{H}, \mathrm{s}, 2 \times \mathrm{Me}), 1.20(3 \mathrm{H}, \mathrm{t}, J=$ $\left.7.5 \mathrm{~Hz}, \mathrm{CH}_{3} \mathrm{Et}\right) .{ }^{13} \mathbf{C}$ NMR (75 MHz, $\left.\mathbf{C D C l}_{3}\right) \delta 151.5$ (1 C, C Ar), 141.7 (1 C, C Ar), 141.3 (1 C, C Ar), 128.0 (2 C, CH Ph), 127.8 (1 C, C Ar), 127.1 (1 C, CH Ar), 126.9 (2 C, CH Ph), 125.5 (1 C, CH Ar), 125.4 (1 C, CH Ar), 115.3 (1 C, C-6), 42.4 (1 C, C-7), 31.1 (2 C, 2 x Me), 24.6 (1 C, $\left.\mathrm{CH}_{2} \mathrm{Et}\right)$, 13.4 (1 C, $\left.\mathrm{CH}_{3} \mathrm{Et}\right)$. HRMS (EI): calculated for $\mathrm{C}_{17} \mathrm{H}_{21} \mathrm{~N}[\mathrm{M}]^{+}$requires $\mathrm{m} / \mathrm{z}$ 239.1674, found $\mathrm{m} / \mathrm{z}$ 239.1671 .

\subsection{2-Benzyl-4-(2-phenylpropan-2-yl)aniline, 3k.}

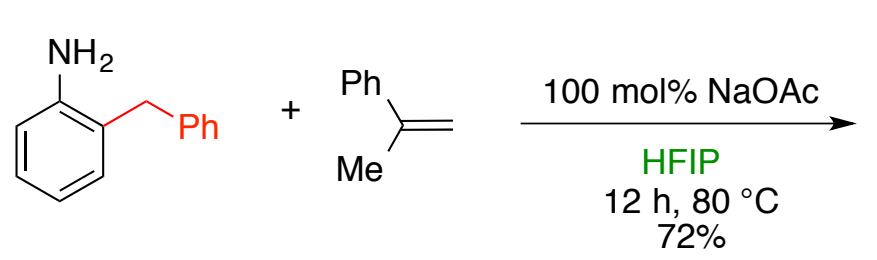

$\mathbf{1 k}$
$(1.0$ equiv.)

$2 a$

(2.0 equiv.)

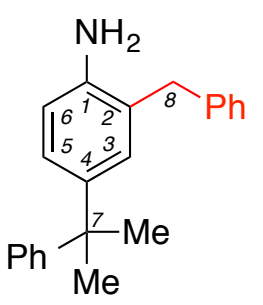

3k

From aniline 1k (37 mg, $0.2 \mathrm{mmol})$, alkene 2a (52 $\mu \mathrm{l}, 0.4 \mathrm{mmol})$ and $\mathrm{NaOAc}(16 \mathrm{mg}, 0.2$ mmol), in $1.0 \mathrm{~mL}$ of HFIP, following the general procedure, aniline $\mathbf{3 k}$ was obtained. Chromatographic purification (gradient elution: 5:95 $\rightarrow$ 30:70 $\mathrm{Et}_{2} \mathrm{O}$ - hexane) gave 3k (43 mg, 72\%), as a yellow oil.

Data for 3k: $\boldsymbol{R}_{f} 0.20$ (25\% $\mathrm{Et}_{2} \mathrm{O}$ - hexane). ${ }^{\mathbf{1}} \mathbf{H}$ NMR (300 $\left.\mathbf{~ M H z}, \mathbf{C D C l}_{3}\right) \delta$ 7.11-7.34 (10 H, m, Ph), 6.94-7.00 (2 H, m, 3-H and 5-H), $6.60(1 \mathrm{H}, \mathrm{d}, J=7.8 \mathrm{~Hz}, 6-\mathrm{H}), 2.92\left(2 \mathrm{H}, \mathrm{s}, 8-\mathrm{H}_{2}\right), 3.37$ (2 H, br s, $\left.\mathrm{NH}_{2}\right), 1.66\left(6 \mathrm{H}, \mathrm{s}, 2\right.$ x Me). ${ }^{13} \mathbf{C}$ NMR (75 MHz, CDCl $) \delta 151.3$ (1 C, C Ar), $142.3(1 \mathrm{C}, \mathrm{C}$ Ar), 141.2 (1 C, C Ar), 139.6 (1 C, C Ar), 129.6 (1 C, CH Ar), 128.6 (2 C, CH Ph), 128.4 (2 C, CH Ph), 127.9 (2 C, CH Ph), 126.8 (2 C, CH Ph), 126.3 (1 C, CH Ar), 126.1 (1 C, CH Ar), 125.4 (1 C, CH Ar), 124.6 (1 C, C Ar), 115.7 (1 C, C-6), 42.2 (1 C, C-7), 38.5 (1 C, C-8), 30.9 (2 C, 2 x Me). HRMS (EI): calculated for $\mathrm{C}_{22} \mathrm{H}_{23} \mathrm{~N}[\mathrm{M}]^{+}$requires $\mathrm{m} / \mathrm{z}, 301.1830$, found $\mathrm{m} / \mathrm{z} 301.1817$. 
3.12. 5-(2-Phenylpropan-2-yl)-[1,1'-biphenyl]-2-amine, 31 .

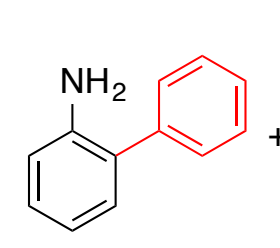

11

(1.0 equiv.)

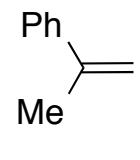

$2 a$

(2.0 equiv.)

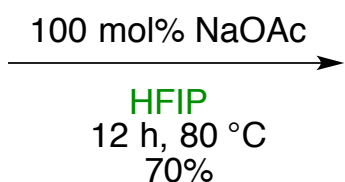

$70 \%$

From aniline 11 (34 mg, $0.2 \mathrm{mmol})$, alkene 2a (52 $\mu \mathrm{l}, 0.4 \mathrm{mmol})$ and $\mathrm{NaOAc}(16 \mathrm{mg}, 0.2$ mmol), in $1.0 \mathrm{~mL}$ of HFIP, following the general procedure, aniline $3 \mathbf{l}$ was obtained. Chromatographic purification (gradient elution: 5:95 $\rightarrow$ 30:70 $\mathrm{Et}_{2} \mathrm{O}$ - hexane) gave $3 \mathrm{l}$ (40 mg, 70\%), as a yellow oil.

Data for 31: $\boldsymbol{R}_{f} 0.25\left(25 \% \mathrm{Et}_{2} \mathrm{O}\right.$ - hexane). ${ }^{1} \mathbf{H}$ NMR (300 $\left.\mathbf{M H z}, \mathbf{C D C l}_{3}\right) \delta$ 7.11-7.48 $(10 \mathrm{H}, \mathrm{m}$, $\mathrm{Ph}), 7.12(1 \mathrm{H}, \mathrm{dd}, J=2.3$ and $0.9 \mathrm{~Hz}, 3-\mathrm{H}), 6.68(1 \mathrm{H}, \mathrm{ddd}, J=8.3,2.4$ and $0.9 \mathrm{~Hz}, 5-\mathrm{H})$, ), 6.68 (1 $\mathrm{H}, \mathrm{dd}, J=8.2$ and $0.9 \mathrm{~Hz}, 6-\mathrm{H}), 3.63\left(2 \mathrm{H}, \mathrm{s}, \mathrm{NH}_{2}\right), 1.67(6 \mathrm{H}, \mathrm{s}, 2 \mathrm{x} \mathrm{Me}) .{ }^{13} \mathbf{C} \mathbf{~ N M R}\left(75 \mathbf{~ M H z}, \mathbf{C D C l}_{3}\right)$ $\delta 151.1$ (1 C, C Ar), 141.1 (1 C, C Ar), 141.0 (1 C, C Ar), 139.9 (1 C, C Ar), 129.2 (2 C, CH Ar), 128.8 (2 C, CH Ar), 128.7 (1 C, CH Ar), 127.9 (2 C, CH Ar), 127.2 (1 C, CH Ar), 127.15 (1 C, CH Ar), 127.14 (1 C, C Ar), 126.8 (2 C, CH Ar), 125.5 (1 C, CH Ar), 115.4 (1 C, C-6), 42.3 (1 C, C-7), $30.9(2 \mathrm{C}, 2 \times \mathrm{Me})$. HRMS (EI): calculated for $\mathrm{C}_{21} \mathrm{H}_{21} \mathrm{~N}[\mathrm{M}]^{+}$requires $\mathrm{m} / \mathrm{z}$ 287.1674, found $\mathrm{m} / \mathrm{z}$ 287.1685 .

3.13. 2-Methoxy-4-(2-phenylpropan-2-yl)aniline, $3 \mathrm{~m}$.

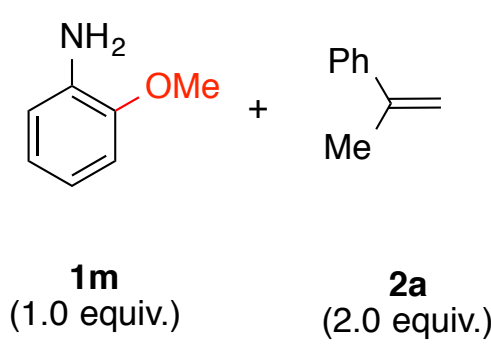

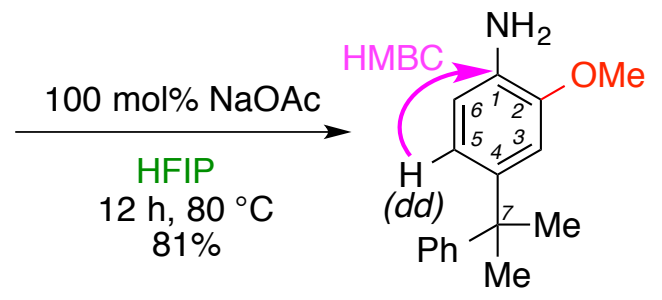

$3 \mathrm{~m}$

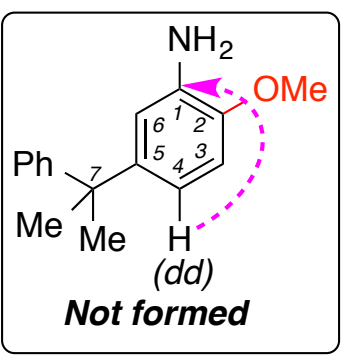

$\mathrm{NH}_{2}$

From aniline 1m (25 mg, $0.2 \mathrm{mmol})$, alkene $2 \mathbf{a}(52 \mu \mathrm{l}, 0.4 \mathrm{mmol})$ and NaOAc (16 mg, 0.2 $\mathrm{mmol}$ ), in $1.0 \mathrm{~mL}$ of HFIP, following the general procedure, aniline $\mathbf{3 m}$ was obtained. Chromatographic purification (gradient elution: 5:95 $\rightarrow$ 70:30 $\mathrm{Et}_{2} \mathrm{O}$ - hexane) gave $3 \mathbf{m}(39 \mathrm{mg}, 81 \%$ ), as a red oil.

Data for 3m: $\boldsymbol{R}_{f} 0.40\left(50 \% \mathrm{Et}_{2} \mathrm{O}\right.$ - hexane). ${ }^{1} \mathbf{H}$ NMR (300 $\left.\mathbf{M H z}, \mathbf{C D C l}_{3}\right) \delta$ 7.15-7.30 (5 H, $\mathrm{m}, \mathrm{Ph}), 6.72(1 \mathrm{H}, \mathrm{dd}, J=8.1$ and $2.0 \mathrm{~Hz}, 5-\mathrm{H}), 6.65(1 \mathrm{H}, \mathrm{d}, J=7.9 \mathrm{~Hz}, 6-\mathrm{H}), 6.64(1 \mathrm{H}, \mathrm{d}, J=2.0$ $\mathrm{Hz}, 3-\mathrm{H}), 3.75(3 \mathrm{H}, \mathrm{s}, \mathrm{OMe}), 3.65\left(2 \mathrm{H}, \mathrm{br} \mathrm{s}, \mathrm{NH}_{2}\right), 1.67\left(6 \mathrm{H}, \mathrm{s}, 2\right.$ x Me). ${ }^{13} \mathrm{C}$ NMR (75 MHz, $\left.\mathbf{C D C l}_{3}\right) \delta 151.3$ (1 C, C Ph), 147.1 (1 C, C-2), 141.4 (1 C, C-4), 133.9 (1 C, C-1), 128.0 (2 C, CH 
Ph), 126.9 (2 C, CH Ph), 125.6 (1 C, CH Ph), 119.2 (1 C, C-5), 114.6 (1 C, C-6), 109.9 (1 C, C-3), 55.6 (1 C, OMe), 42.7 (1 C, C-7), 31.1 (2 C, 2 x Me). ${ }^{1} \mathrm{H}(400 \mathrm{MHz})-{ }^{13} \mathrm{C}(\mathbf{1 0 0} \mathrm{MHz}) \mathbf{H M B C}$ $\left(\mathbf{C D C l}_{3}\right)$ : between 6-H and C-1, among other cross-peaks. HRMS (EI): calculated for $\mathrm{C}_{16} \mathrm{H}_{19} \mathrm{NO}[\mathrm{M}]^{+}$ requires $\mathrm{m} / \mathrm{z}, 241.1467$, found $\mathrm{m} / \mathrm{z}, 241.1455$.

3.14. 4-(2-Phenylpropan-2-yl)benzene-1,2-diamine, 3n.<smiles>C=C(C)Pc1ccccc1</smiles>

1n (1.0 equiv.)

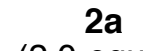

(2.0 equiv.)

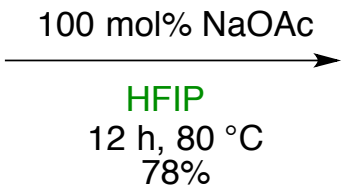

$78 \%$

From aniline 1n (22 mg, $0.2 \mathrm{mmol})$, alkene 2a (52 $\mu \mathrm{l}, 0.4 \mathrm{mmol})$ and $\mathrm{NaOAc}(16 \mathrm{mg}, 0.2$ $\mathrm{mmol}$ ), in $1.0 \mathrm{~mL}$ of HFIP, following the general procedure, aniline $\mathbf{3 n}$ was obtained. Chromatographic purification (gradient elution: 5:95 $\rightarrow$ 70:30 $\mathrm{Et}_{2} \mathrm{O}$ - hexane) gave 3n (35 mg, 78\%), as a red oil.

Data for 3n: $\boldsymbol{R}_{f} 0.30\left(50 \% \mathrm{Et}_{2} \mathrm{O}-\right.$ hexane $) .{ }^{1} \mathbf{H} \mathbf{N M R}\left(\mathbf{3 0 0} \mathbf{M H z}, \mathbf{C D C l}_{3}\right) \delta 7.12-7.31(5 \mathrm{H}, \mathrm{m}$, $\mathrm{Ph}), 6.65-6.72\left(2 \mathrm{H}, \mathrm{m}, 3-\mathrm{H}\right.$ and 6-H), $6.53(1 \mathrm{H}, \mathrm{d}, J=1.2 \mathrm{~Hz}, 5-\mathrm{H}), 3.86\left(3 \mathrm{H}, \mathrm{br} \mathrm{s}, \mathrm{OH}\right.$ and $\left.\mathrm{NH}_{2}\right)$, $1.61(6 \mathrm{H}, \mathrm{s}, 2 \mathrm{x} \mathrm{Me}) .{ }^{13} \mathbf{C}$ NMR (75 MHz, $\left.\mathbf{C D C l}_{3}\right) \delta 151.2$ (1 C, C Ar), 144.1 (1 C, C Ar), 143.1 (1 C, C Ar), 131.7 (1 C, C Ar), 128.1 (2 C, CH Ar), 126.9 (2 C, CH Ar), 125.7 (1 C, CH Ph), 119.4 and 117.1 and 114.6 (3 C, C-3 and C-5 and C-6), 61.5 (1 C, C-7), 46.5 (1 C, C-6), 42.3 (1 C, C-5), 31.0 (2 C, 2 x Me). HRMS (EI): calculated for $\mathrm{C}_{15} \mathrm{H}_{17} \mathrm{NO}[\mathrm{M}]^{+}$requires $m / z$ 227.1310, found $m / z$ 227.1318.

\subsection{2-Amino-5-(2-phenylpropan-2-yl)phenol, 3o.}<smiles>C=C(C)Pc1ccccc1</smiles>

10

(1.0 equiv.)

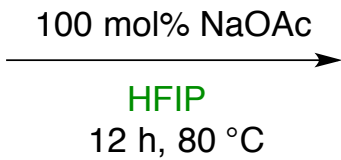

$71 \%$

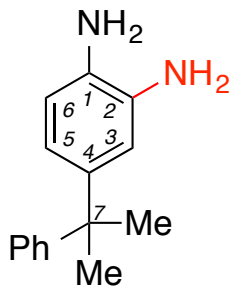

30

From aniline $10(22 \mathrm{mg}, 0.2 \mathrm{mmol})$, alkene $2 \mathbf{a}(52 \mu \mathrm{l}, 0.4 \mathrm{mmol})$ and $\mathrm{NaOAc}(16 \mathrm{mg}, 0.2$ $\mathrm{mmol}$ ), in $1.0 \mathrm{~mL}$ of HFIP, following the general procedure, aniline 30 was obtained. Chromatographic purification (gradient elution: 5:95 $\rightarrow$ 70:30 $\mathrm{Et}_{2} \mathrm{O}$ - hexane) gave 30 (32 $\mathrm{mg}, 71 \%$ ), as a red foam.

Data for 3o: $\boldsymbol{R}_{f} 0.25\left(50 \% \mathrm{Et}_{2} \mathrm{O}\right.$ - hexane). ${ }^{1} \mathbf{H}$ NMR (300 $\left.\mathbf{M H z}, \mathbf{C D C l}_{3}\right) \delta$ 7.03-7.24 (5 H, m, $\mathrm{Ph}), 6.54\left(2 \mathrm{H}, \mathrm{d}, J=1.3 \mathrm{~Hz}, 3-\mathrm{H}\right.$ and 6-H), $6.46(1 \mathrm{H}, \mathrm{t}, J=1.2 \mathrm{~Hz}, 5-\mathrm{H}), 3.06\left(2 \mathrm{H}, \mathrm{br} \mathrm{s}, \mathrm{NH}_{2}\right), 1.55$ 
(6 H, s, 2 x Me). $\left.{ }^{13} \mathbf{C ~ N M R ~ ( 7 5 ~ M H z , ~} \mathbf{C D C l}_{3}\right) \delta 151.4$ (1 C, C Ar), 143.2 (1 C, C Ar), 134.5 (1 C, C Ar), 132.4 (1 C, C Ar), 128.0 (2 C, CH Ph), 126.9 (2 C, CH Ph), 125.5 (1 C, CH Ph), 118.5 and 116.6 and 115.9 (3 C, C-3 and C-5 and C-6), 42.4 (1 C, C-7), 31.0 (2 C, 2 x Me). HRMS (EI): calculated for $\mathrm{C}_{15} \mathrm{H}_{18} \mathrm{~N}_{2}[\mathrm{M}]^{+}$requires $m / z, 226.1470$, found $m / z, 226.1473$.

\subsection{2-Fluoro- $N$-methyl-4-(2-phenylpropan-2-yl)aniline, $3 p$.}

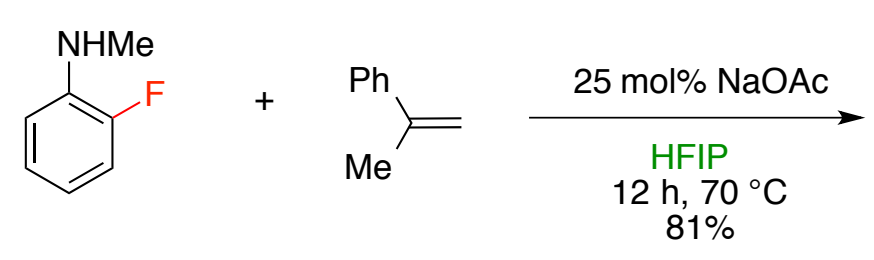

$(1.0$ equiv.)
(2.0 equiv.)

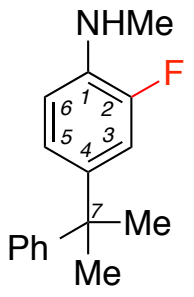

$3 p$

From aniline 1p (25 mg, $0.2 \mathrm{mmol})$, alkene 2a (52 $\mu \mathrm{l}, 0.4 \mathrm{mmol})$ and $\mathrm{NaOAc}(4.0 \mathrm{mg}, 0.05$ $\mathrm{mmol}$ ), in $1.0 \mathrm{~mL}$ of HFIP, following the general procedure, aniline $\mathbf{3 p}$ was obtained. Chromatographic purification (gradient elution: 5:95 $\rightarrow$ 30:70 $\mathrm{Et}_{2} \mathrm{O}$ - hexane) gave 3p (40 mg, 81\%), as a colourless oil.

Data for 3p: $\boldsymbol{R}_{f} 0.30\left(10 \% \mathrm{Et}_{2} \mathrm{O}\right.$ - hexane). ${ }^{\mathbf{1}} \mathbf{H} \mathbf{N M R}\left(300 \mathbf{M H z}, \mathbf{C D C l}_{3}\right) \delta$ 7.13-7.33 (5 H, m, $\mathrm{Ph}), 6.92\left(1 \mathrm{H}\right.$, ddd, $J_{5-6}=8.3 \mathrm{~Hz}$ and $J_{5-3}=2.2 \mathrm{~Hz}$ and $\left.J_{5-F}=0.9 \mathrm{~Hz}, 5-\mathrm{H}\right), 6.85\left(1 \mathrm{H}, \mathrm{dd}, J_{3-F}=13.6\right.$ $\mathrm{Hz}$ and $\left.J_{3-5}=2.1 \mathrm{~Hz}, 3-\mathrm{H}\right), 6.61\left(1 \mathrm{H}, \mathrm{dd}, J_{6-F}=9.1 \mathrm{~Hz}\right.$ and $\left.J_{6-5}=8.5 \mathrm{~Hz}, 6-\mathrm{H}\right), 3.82(1 \mathrm{H}, \mathrm{br} \mathrm{s}, \mathrm{NH})$, $2.86(3 \mathrm{H}, \mathrm{s}, \mathrm{NMe}), 1.65$ (6 H, s, 2 x Me). ${ }^{13} \mathbf{C}$ NMR (75 MHz, $\left.\mathbf{C D C l}_{3}\right) \delta 151.4\left(1 \mathrm{C}, \mathrm{d}, J_{2-F}=238.0\right.$ $\mathrm{Hz}, \mathrm{C}-2), 150.9$ (1 C, C Ph), 139.8 (1 C, d, $\left.J_{4-F}=5.3 \mathrm{~Hz}, \mathrm{C} 4\right), 135.5$ (1 C, d, J J-F $\left.=12.0 \mathrm{~Hz}, \mathrm{C}-1\right)$, 128.1 (2 C, CH Ph), 126.8 (2 C, CH Ph), 125.7 (1 C, CH Ph), 122.5 (1 C, d, J J-F $=3.0 \mathrm{~Hz}, \mathrm{C}-5), 113.3$ $\left(1 \mathrm{C}, \mathrm{d}, J_{3-F}=18.8 \mathrm{~Hz}, \mathrm{C}-3\right), 111.1\left(1 \mathrm{C}, \mathrm{d}, J_{6-F}=3.8 \mathrm{~Hz}, \mathrm{C}-6\right), 42.3\left(1 \mathrm{C}, \mathrm{d}, J_{7-F}=1.4 \mathrm{~Hz}, \mathrm{C}-7\right), 30.9$ (2 C, $2 \times \mathrm{Me}), 30.5$ (1 C, NMe). HRMS (ESI): calculated for $\mathrm{C}_{16} \mathrm{H}_{19} \mathrm{NF}[\mathrm{M}+\mathrm{H}]^{+}$requires $\mathrm{m} / \mathrm{z}$ 244.1496 , found $m / z, 244.1494$.

\subsection{2-Chloro- $N$-methyl-4-(2-phenylpropan-2-yl)aniline, 3q.}<smiles>CNc1ccccc1Cl</smiles>

$1 q$ (1.0 equiv.)<smiles>C=C(C)Pc1ccccc1</smiles>

2a (2.0 equiv.)

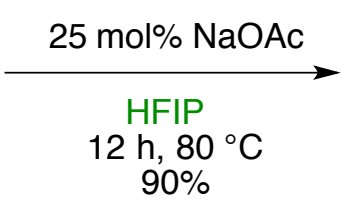

$90 \%$

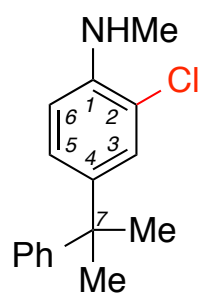

$3 q$

From aniline $1 \mathbf{1 q}(28 \mathrm{mg}, 0.2 \mathrm{mmol})$, alkene $2 \mathbf{a}(52 \mu \mathrm{l}, 0.4 \mathrm{mmol})$ and $\mathrm{NaOAc}(4.0 \mathrm{mg}, 0.05$ mmol), in $1.0 \mathrm{~mL}$ of HFIP, following the general procedure, aniline $\mathbf{3 q}$ was obtained. 
Chromatographic purification (gradient elution: $5: 95 \rightarrow 30: 70 \mathrm{Et}_{2} \mathrm{O}-$ hexane) gave $\mathbf{3 q}(47 \mathrm{mg}, 90 \%$ ), as a colourless oil.

Data for 3q: $\boldsymbol{R}_{f} 0.40\left(25 \% \mathrm{Et}_{2} \mathrm{O}\right.$ - hexane). ${ }^{\mathbf{1}} \mathbf{H}$ NMR (300 $\left.\mathbf{M H z}, \mathbf{C D C l}_{3}\right) \delta$ 7.15-7.31 (5 H, m, $\mathrm{Ph}), 7.15(1 \mathrm{H}, \mathrm{d}, J=2.2 \mathrm{~Hz}, 3-\mathrm{H}), 7.01(1 \mathrm{H}, \mathrm{dd}, J=8.4$ and $2.3 \mathrm{~Hz}, 5-\mathrm{H}), 6.56(1 \mathrm{H}, \mathrm{d}, J=8.5 \mathrm{~Hz}$, 6-H), 4.21 (1 H, br s, NH), 2.86 (3 H, s, NMe), 1.63 (6 H, s, 2 x Me). ${ }^{13}$ C NMR (75 MHz, CDCl $) \delta$ 150.8 (1 C, C Ar), 143.0 (1 C, C Ar), 140.0 (1 C, C Ar), 128.1 (2 C, CH Ph), 127.5 (1 C, CH Ar), 126.8 (2 C, CH Ph), 126.4 (1 C, CH Ar), 125.7 (1 C, CH Ar), 118.9 (2 C, C Ar), 110.4 (2 C, C-6), 42.3 (1 C, C-7), 30.9 (2 C, 2 x Me), 30.6 (1 C, NMe). HRMS (ESI): calculated for $\mathrm{C}_{16} \mathrm{H}_{19} \mathrm{NCl}[\mathrm{M}+\mathrm{H}]^{+}$ requires $m / z, 260.1200$, found $m / z, 260.1202$.

3.18. 2-Amino-5-(2-phenylpropan-2-yl)benzoic acid, 3 r.

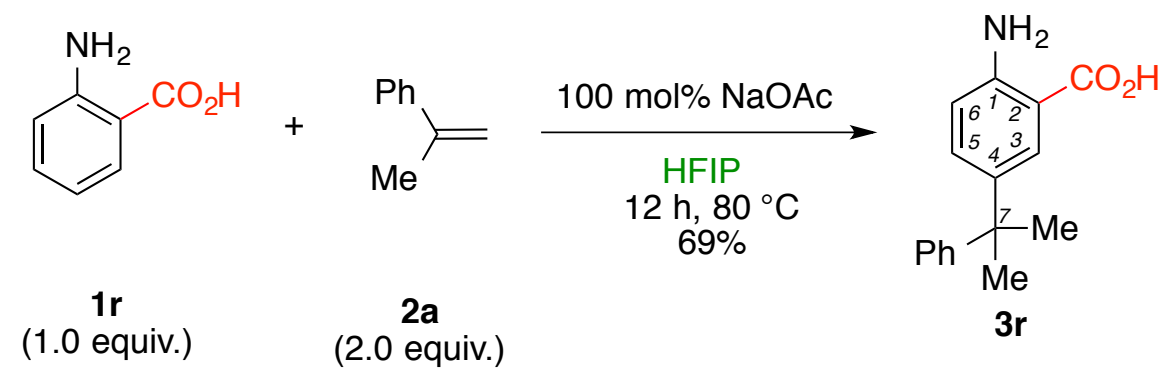

From aniline 1r (27 mg, $0.2 \mathrm{mmol})$, alkene 2a (52 $\mu \mathrm{l}, 0.4 \mathrm{mmol})$ and NaOAc (4.0 mg, 0.05 mmol), in $1.0 \mathrm{~mL}$ of HFIP, following the general procedure, aniline $\mathbf{3 r}$ was obtained. Chromatographic purification (gradient elution: $1: 99 \rightarrow 10: 90 \mathrm{MeOH}-\mathrm{CH}_{2} \mathrm{Cl}_{2}$ ) gave $3 \mathbf{r}(35 \mathrm{mg}$, $69 \%)$, as a yellow foam.

Data for 3r: $\boldsymbol{R}_{f} 0.40\left(5 \% \mathrm{MeOH}-\mathrm{CH}_{2} \mathrm{Cl}_{2}\right) .{ }^{1} \mathbf{H}$ NMR (300 MHz, $\mathbf{C D}_{3}$ OD) $\delta 7.80(1 \mathrm{H}, \mathrm{d}, J=$ $2.4 \mathrm{~Hz}, 3-\mathrm{H}), 7.15-7.31(5 \mathrm{H}, \mathrm{m}, \mathrm{Ph}), 7.02(1 \mathrm{H}, \mathrm{dd}, J=8.7$ and $2.4 \mathrm{~Hz}, 5-\mathrm{H}), 6.64(2 \mathrm{H}, \mathrm{d}, J=8.7$ $\mathrm{Hz}, 6-\mathrm{H}), 1.62\left(6 \mathrm{H}, \mathrm{s}, 2\right.$ x Me). ${ }^{13} \mathrm{C}$ NMR (75 MHz, CD $\left.\mathbf{O D}\right) \delta 171.8\left(1 \mathrm{C}, \mathrm{CO}_{2} \mathrm{H}\right), 152.1(1 \mathrm{C}, \mathrm{C}$ Ar), 150.6 (1 C, C Ar), 139.2 (1 C, C Ar), 134.6 (1 C, C-5), 129.6 (1 C, C-6), 129.0 (2 C, CH Ph), 127.7 (2 C, CH Ph), 126.6 (1 C, CH Ph), 117.9 (1 C, C-3), 111.1 (1 C, C Ar), 43.0 (1 C, C-7), 31.2 (2 C, $2 \times \mathrm{Me}$ ). HRMS (ESI): calculated for $\mathrm{C}_{16} \mathrm{H}_{16} \mathrm{NO}[\mathrm{M}+\mathrm{H}]^{+}$requires $\mathrm{m} / \mathrm{z}$ 238.1226, found $\mathrm{m} / \mathrm{z}$ 238.1237 .

3.19. $\quad$ N,3-Dimethyl-4-(2-phenylpropan-2-yl)aniline, 3s.

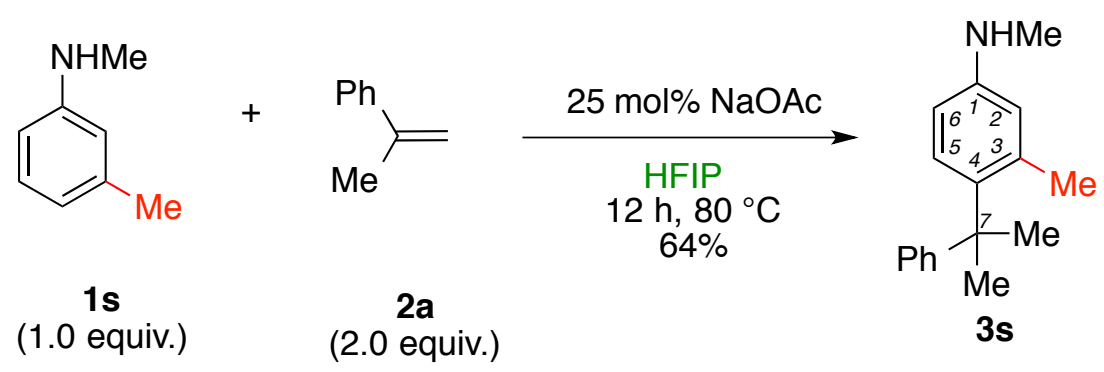


From aniline $1 \mathrm{~s}(24 \mathrm{mg}, 0.2 \mathrm{mmol})$, alkene 2a (52 $\mu \mathrm{l}, 0.4 \mathrm{mmol})$ and $\mathrm{NaOAc}(4.0 \mathrm{mg}, 0.05$ $\mathrm{mmol}$ ), in $1.0 \mathrm{~mL}$ of HFIP, following the general procedure, aniline $3 \mathrm{~s}$ was obtained. Chromatographic purification (gradient elution: 5:95 $\rightarrow$ 30:70 $\mathrm{Et}_{2} \mathrm{O}$ - hexane) gave 3s (30 mg, 64\%), as a yellow oil.

Data for 3s: $\boldsymbol{R}_{f} 0.40\left(25 \% \mathrm{Et}_{2} \mathrm{O}\right.$ - hexane). ${ }^{1} \mathbf{H}$ NMR (300 $\left.\mathbf{~ M H z}, \mathbf{C D C l}_{3}\right) \delta 7.43(1 \mathrm{H}, \mathrm{d}, J=$ 8.5 Hz, 5-H), 7.11-7.32 (5 H, m, Ph), $6.52(1 \mathrm{H}, \mathrm{dd}, J=8.4$ and $2.8 \mathrm{~Hz}, 6-\mathrm{H}), 6.38(1 \mathrm{H}, \mathrm{d}, J=2.7$ Hz, 2-H), 3.54 (1 H, br s, NH), 2.85 (3 H, s, NMe), 1.76 (3 H, s, Me), 1.68 (6 H, s, 2 x Me). ${ }^{13}$ C NMR (75 MHz, $\mathbf{C D C l}_{3}$ ) $\delta 151.9$ (1 C, C Ar), 147.7 (1 C, C Ar), 137.9 (1 C, C Ar), 136.8 (1 C, C Ar), 128.2 (2 C, CH Ph), 127.1 (1 C, CH Ar), 126.0 (2 C, CH Ph), 125.2 (1 C, CH Ar), 116.7 (1 C, C-6), 109.1 (1 C, C-2), 42.9 (1 C, C-7), 31.3 (2 C, 2 x Me), 30.9 (1 C, NMe), 22.0 (1 C, Me). HRMS (ESI): calculated for $\mathrm{C}_{17} \mathrm{H}_{22} \mathrm{~N}[\mathrm{M}+\mathrm{H}]^{+}$requires $m / z$ 240.1746, found $m / z 240.1754$.

\subsection{3-Methoxy- $N$-methyl-4-(2-phenylpropan-2-yl)aniline, $3 t$.}

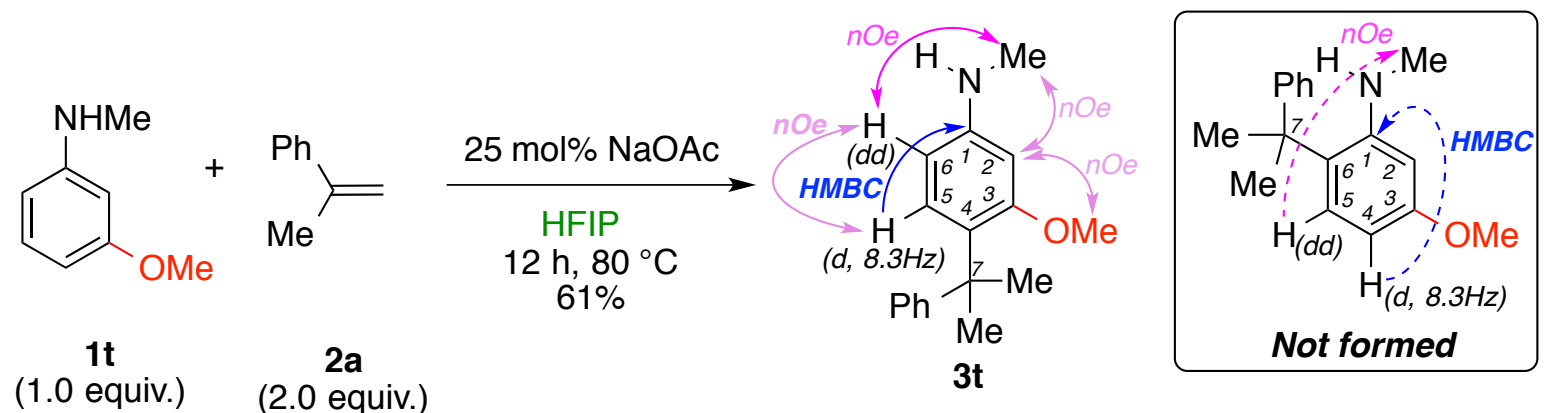

From aniline 1t (28 mg, $0.2 \mathrm{mmol})$, alkene 2a (52 $\mu 1,0.4 \mathrm{mmol})$ and $\mathrm{NaOAc}(4.0 \mathrm{mg}, 0.05$ mmol), in $1.0 \mathrm{~mL}$ of HFIP, following the general procedure, aniline $\mathbf{3 t}$ was obtained. Chromatographic purification (gradient elution: 5:95 $\rightarrow 30: 70 \mathrm{Et}_{2} \mathrm{O}$ - hexane) gave $3 \mathrm{t}(31 \mathrm{mg}, 61 \%$ ), as a white foam.

Data for 3t: $\boldsymbol{R}_{f} 0.30\left(25 \% \mathrm{Et}_{2} \mathrm{O}\right.$ - hexane). ${ }^{1} \mathbf{H}$ NMR (300 MHz, $\left.\mathbf{C D C l}_{3}\right) \delta 7.26(1 \mathrm{H}, \mathrm{d}, J=$ 8.3 Hz, 5-H), 7.04-7.22 (5 H, m, Ph), $6.25(1 \mathrm{H}, \mathrm{dd}, J=8.3$ and $2.4 \mathrm{~Hz}, 6-\mathrm{H}), 6.11(1 \mathrm{H}, \mathrm{d}, J=2.3$ $\mathrm{Hz}, 2-\mathrm{H}), 3.65$ (1 H, br s, NH), 3.31 (3 H, s, OMe), 2.84 (3 H, s, NMe), 1.64 (6 H, s, 2 x Me). ${ }^{13} \mathrm{C}$ NMR (75 MHz, CDCl $) \delta 159.1$ (1 C, C-3), 152.4 (1 C, C Ph), 149.3 (1 C, C-1), 128.2 (1 C, C-4), 127.6 (2 C, CH Ph), 127.5 (1 C, C-5), 125.7 (2 C, CH Ph), 124.7 (1 C, CH Ph), 103.7 (1 C, C-6), 98.8 (1 C, C-2), 55.3 (1 C, OMe), 41.4 (1 C , C-7), 31.1 (1 C, NMe), 30.0 (2 C, 2 x Me). ${ }^{1} \mathrm{H}$ (400 MHz) ${ }^{13} \mathrm{C}(100 \mathrm{MHz}) \mathrm{HMBC}\left(\mathrm{CDCl}_{3}\right)$ : between 5-H and C-1, among other cross-peaks. NOESY-2D (400 MHz, $\mathbf{C D C l}_{3}$ ): between 6-H and NMe, among other cross-peaks. HRMS (ESI): calculated for $\mathrm{C}_{17} \mathrm{H}_{22} \mathrm{NO}[\mathrm{M}+\mathrm{H}]^{+}$requires $m / z, 256.1695$, found $m / z 256.1695$. 
3.21. 3-Fluoro- $N$-methyl-4-(2-phenylpropan-2-yl)aniline, $3 u$.<smiles>CNc1cccc(F)c1</smiles>

$1 \mathrm{u}$ (1.0 equiv.)<smiles>C=C(C)Pc1ccccc1</smiles>

$2 a$ (2.0 equiv.)

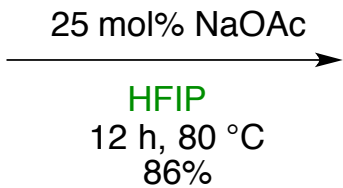

$86 \%$

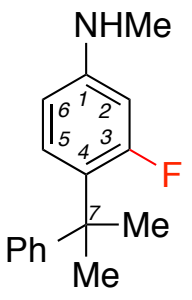

3u

From aniline $\mathbf{1 u}(25 \mathrm{mg}, 0.2 \mathrm{mmol})$, alkene $\mathbf{2 a}(52 \mu \mathrm{l}, 0.4 \mathrm{mmol})$ and $\mathrm{NaOAc}(4.0 \mathrm{mg}, 0.05$ $\mathrm{mmol}$ ), in $1.0 \mathrm{~mL}$ of HFIP, following the general procedure, aniline $\mathbf{3 u}$ was obtained. Chromatographic purification (gradient elution: 5:95 $\rightarrow$ 30:70 $\mathrm{Et}_{2} \mathrm{O}$ - hexane) gave $3 \mathbf{u}(42 \mathrm{mg}, 86 \%$ ), as a yellow oil.

Data for 3u: $\boldsymbol{R}_{f} 0.40\left(25 \% \mathrm{Et}_{2} \mathrm{O}\right.$ - hexane). ${ }^{\mathbf{1}} \mathbf{H} \mathbf{N M R}\left(300 \mathbf{~ M H z}, \mathbf{C D C l}_{3}\right) \delta$ 7.07-7.33 (6 H, m, $5 \mathrm{x} \mathrm{CH} \mathrm{Ph}$ and 5-H), $6.37\left(1 \mathrm{H}, \mathrm{dd}, J_{6-5}=8.5 \mathrm{~Hz}\right.$ and $\left.J_{6-2}=2.5 \mathrm{~Hz}, 6-\mathrm{H}\right), 6.21\left(1 \mathrm{H}, \mathrm{dd}, J_{2-F}=14.1 \mathrm{~Hz}\right.$ and $\left.J_{2-6}=2.4 \mathrm{~Hz}, 2-\mathrm{H}\right), 3.72\left(1 \mathrm{H}\right.$, br s, NH), $2.81(3 \mathrm{H}, \mathrm{s}, \mathrm{NMe}), 1.67(6 \mathrm{H}, \mathrm{s}, 2 \mathrm{x} \mathrm{Me}) .{ }^{13} \mathrm{C}$ NMR $(75$ MHz, $\left.\mathbf{C D C l}_{3}\right) \delta 162.3\left(1 \mathrm{C}, \mathrm{d}, J_{3-F}=246.7 \mathrm{~Hz}, \mathrm{C}-3\right), 150.9(1 \mathrm{C}, \mathrm{C} \mathrm{Ph}), 149.7\left(1 \mathrm{C}, \mathrm{d}, J_{1-F}=11.1 \mathrm{~Hz}\right.$, C 1), $128.1\left(1 \mathrm{C}, \mathrm{d}, J_{5-F}=7.1 \mathrm{~Hz}, \mathrm{C}-5\right), 128.0(2 \mathrm{C}, \mathrm{CH} \mathrm{Ph}), 125.9$ (2 C, d, $\left.J_{H-F}=1.0 \mathrm{~Hz}, \mathrm{CH} \mathrm{Ph}\right)$, 125.5 (1 C, $\mathrm{CH} \mathrm{Ph}), 125.4\left(1 \mathrm{C}, \mathrm{d}, J_{4-F}=12.5 \mathrm{~Hz}, \mathrm{C}-4\right), 107.5\left(1 \mathrm{C}, \mathrm{d}, J_{6-F}=2.4 \mathrm{~Hz}, \mathrm{C}-6\right), 100.6(1 \mathrm{C}$, $\left.\mathrm{d}, J_{2-F}=27.1 \mathrm{~Hz}, \mathrm{C}-2\right), 40.9\left(1 \mathrm{C}, \mathrm{d}, J_{7-F}=1.2 \mathrm{~Hz}, \mathrm{C}-7\right), 30.9(1 \mathrm{C}, \mathrm{NMe}), 30.1\left(2 \mathrm{C}, \mathrm{d}, J_{M e-F}=2.2 \mathrm{~Hz}\right.$, 2 x Me). HRMS (ESI): calculated for $\mathrm{C}_{16} \mathrm{H}_{19} \mathrm{NF}[\mathrm{M}+\mathrm{H}]^{+}$requires $m / z$ 244.1496, found $\mathrm{m} / z, 244.1499$.

\subsection{3-Chloro- $N$-methyl-4-(2-phenylpropan-2-yl)aniline, $3 \mathrm{v}$.}<smiles>CNc1cccc(Cl)c1</smiles>

$1 \mathrm{v}$ (1.0 equiv.)

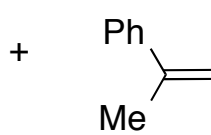

$2 a$

(2.0 equiv.)

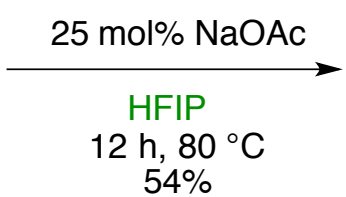
$54 \%$

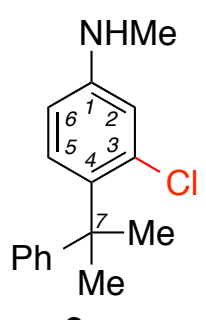

$3 v$

From aniline $\mathbf{1 v}(28 \mathrm{mg}, 0.2 \mathrm{mmol})$, alkene $2 \mathbf{a}(52 \mu \mathrm{l}, 0.4 \mathrm{mmol})$ and $\mathrm{NaOAc}(4.0 \mathrm{mg}, 0.05$ mmol), in $1.0 \mathrm{~mL}$ of HFIP, following the general procedure, aniline $\mathbf{3 v}$ was obtained. Chromatographic purification (gradient elution: 5:95 $\rightarrow$ 30:70 $\mathrm{Et}_{2} \mathrm{O}$ - hexane) gave 3v (28 mg, 54\%), as a yellow foam and starting material $\mathbf{1 v}(9 \mathrm{mg}, 32 \%)$ recovered.

Data for 3v: $\boldsymbol{R}_{f} 0.30\left(25 \% \mathrm{Et}_{2} \mathrm{O}\right.$ - hexane). ${ }^{1} \mathbf{H}$ NMR (300 MHz, $\left.\mathbf{C D C l}_{3}\right) \delta 7.42(1 \mathrm{H}, \mathrm{d}, J=$ $8.8 \mathrm{~Hz}, 5-\mathrm{H}), 7.11-7.30(5 \mathrm{H}, \mathrm{m}, \mathrm{Ph}), 6.56(1 \mathrm{H}, \mathrm{d}, J=2.4 \mathrm{~Hz}, 2-\mathrm{H}), 6.54(1 \mathrm{H}, \mathrm{dd}, J=8.1 \mathrm{and} 2.6 \mathrm{~Hz}$, 6-H), $3.74\left(1 \mathrm{H}\right.$, br s, NH), $2.83(3 \mathrm{H}, \mathrm{s}, \mathrm{NMe}), 1.71(6 \mathrm{H}, \mathrm{s}, 2 \mathrm{x} \mathrm{Me}) .{ }^{13} \mathbf{C}$ NMR $\left(75 \mathbf{M H z}, \mathbf{C D C l}_{3}\right) \delta$ 150.8 (1 C, C Ar), 148.7 (1 C, C Ar), 135.1 (1 C, C Ar), 134.9 (1 C, C Ar), 128.6 (1 C, CH Ar), 128.0 
(2 C, CH Ph), 126.0 (2 C, CH Ph), 125.2 (1 C, CH Ar), 115.4 (1 C, C-6), 110.6 (1 C, C-2), 43.1 (1 C, C-7), 30.8 ( $1 \mathrm{C}, \mathrm{NMe}), 30.5$ (2 C, 2 x Me). HRMS (ESI): calculated for $\mathrm{C}_{16} \mathrm{H}_{19} \mathrm{NCl}[\mathrm{M}+\mathrm{H}]^{+}$requires $\mathrm{m} / \mathrm{z} 260.1200$, found $\mathrm{m} / \mathrm{z} 260.1210$.

3.23. 3-Bromo- $N$-methyl-4-(2-phenylpropan-2-yl)aniline, $3 w$.<smiles>CNc1cccc(Br)c1</smiles>

$1 w$ (1.0 equiv.)<smiles>C=C(C)Pc1ccccc1</smiles>

2a

(2.0 equiv.)

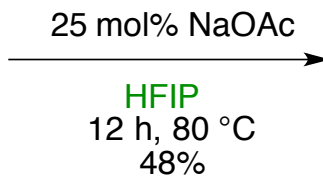
$48 \%$

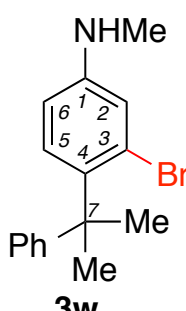

$3 w$

From aniline 1w (38 mg, $0.2 \mathrm{mmol})$, alkene $2 \mathbf{a}(52 \mu \mathrm{l}, 0.4 \mathrm{mmol})$ and $\mathrm{NaOAc}(4.0 \mathrm{mg}, 0.05$ $\mathrm{mmol}$ ), in $1.0 \mathrm{~mL}$ of HFIP, following the general procedure, aniline $\mathbf{3 w}$ was obtained. Chromatographic purification (gradient elution: 5:95 $\rightarrow$ 30:70 $\mathrm{Et}_{2} \mathrm{O}$ - hexane) gave 3w (29 mg, 48\%), as a yellow foam and starting material $\mathbf{1 w}(10 \mathrm{mg}, 26 \%)$ recovered.

Data for 3w: $\boldsymbol{R}_{f} 0.35\left(25 \% \mathrm{Et}_{2} \mathrm{O}\right.$ - hexane). ${ }^{1} \mathbf{H} \mathbf{N M R}\left(300 \mathbf{M H z}, \mathbf{C D C l}_{3}\right) \delta 7.44(1 \mathrm{H}, \mathrm{d}, J=$ $8.6 \mathrm{~Hz}, 5-\mathrm{H}), 7.11-7.33(5 \mathrm{H}, \mathrm{m}, \mathrm{Ph}), 6.81(1 \mathrm{H}, \mathrm{d}, J=2.6 \mathrm{~Hz}, 2-\mathrm{H}), 6.59(1 \mathrm{H}, \mathrm{dd}, J=8.6$ and 2.6

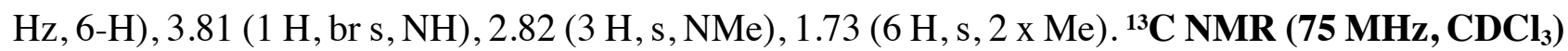
$\delta 150.7$ (1 C, C Ar), 148.6 (1 C, C Ar), 136.2 (1 C, C Ar), 128.7 (1 C, CH Ar), 128.1 (2 C, CH Ph), 126.4 (2 C, CH Ph), 125.2 (1 C, CH Ar), 124.9 (1 C, C Ar), 119.1 (2 C, C-2), 111.1 (1 C, C-6), 44.1 (1 C, C-7), 30.8 (3 C, NMe and $2 \times$ Me). HRMS (ESI): calculated for $\mathrm{C}_{16} \mathrm{H}_{19} \mathrm{NBr}[\mathrm{M}+\mathrm{H}]^{+}$requires $\mathrm{m} / \mathrm{z}$ 304.0695, found $\mathrm{m} / \mathrm{z} 304.0701$.

3.24. 2,6-Diisopropyl-4-(2-phenylpropan-2-yl)aniline, $3 x$.<smiles></smiles>

$1 x$ (1.0 equiv.)

2a

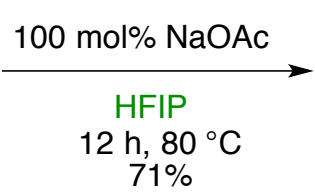

(2.0 equiv.)

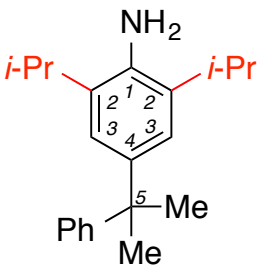

$3 \mathbf{x}$

From aniline 1x (36 mg, $0.2 \mathrm{mmol})$, alkene 2a (52 $\mu \mathrm{l}, 0.4 \mathrm{mmol})$ and $\mathrm{NaOAc}(16 \mathrm{mg}, 0.2$ $\mathrm{mmol}$ ), in $1.0 \mathrm{~mL}$ of HFIP, following the general procedure, aniline $\mathbf{3 x}$ was obtained. Chromatographic purification (gradient elution: 5:95 $\rightarrow$ 30:70 $\mathrm{Et}_{2} \mathrm{O}$ - hexane) gave 3x (42 m, 71\%), as a yellow oil.

Data for 3x: $\boldsymbol{R}_{f} 0.30\left(25 \% \mathrm{Et}_{2} \mathrm{O}\right.$ - hexane). ${ }^{1} \mathbf{H}$ NMR (300 $\left.\mathbf{M H z}, \mathbf{C D C l}_{3}\right) \delta 7.09-7.41(5 \mathrm{H}, \mathrm{m}$, Ph), $6.92(2 \mathrm{H}, \mathrm{s}, 3-\mathrm{H}), 3.68\left(2 \mathrm{H}\right.$, br s, $\left.\mathrm{NH}_{2}\right), 2.94(2 \mathrm{H}$, sept, $J=6.7 \mathrm{~Hz}, 2$ x CH $i$-Pr), 1.69 (6 H, s, $2 \times \mathrm{Me}), 1.23\left(12 \mathrm{H}, \mathrm{d}, J=6.8 \mathrm{~Hz}, 4 \times \mathrm{CH}_{3} i\right.$-Pr). ${ }^{13} \mathbf{C} \mathbf{N M R}\left(75 \mathbf{M H z}, \mathbf{C D C l}_{3}\right) \delta 151.6(1 \mathrm{C}, \mathrm{C} \mathrm{Ar})$, 
140.6 (1 C, C Ar), 137.9 (1 C, C Ar), 132.1 (2 C, C-2), 127.9 (2 C, CH Ar), 126.9 (2 C, CH Ar), 125.4 (1 C, $\mathrm{CH} \mathrm{Ph}), 121.5$ (2 C, CH Ar), 42.8 (1 C, C-5), 31.2 (2 C, 2 x Me), 28.3 (2 C, 2 x CH i-Pr), 22.7 (4 C, 4 x $\mathrm{CH}_{3} i$-Pr). HRMS (ESI): calculated for $\mathrm{C}_{21} \mathrm{H}_{30} \mathrm{~N}[\mathrm{M}+\mathrm{H}]^{+}$requires $\mathrm{m} / z$ 296.2373, found $\mathrm{m} / \mathrm{z}$ 296.2367.

3.25. 2-Chloro-6-methyl-4-(2-phenylpropan-2-yl)aniline, $3 y$.<smiles>C=C(C)Pc1ccccc1</smiles>

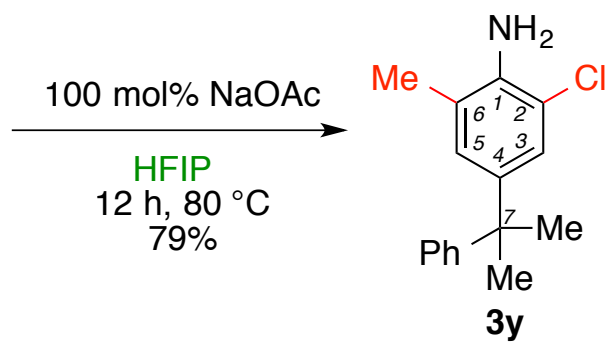

From aniline 1y (28 mg, $0.2 \mathrm{mmol})$, alkene $\mathbf{2 a}(52 \mu \mathrm{l}, 0.4 \mathrm{mmol})$ and $\mathrm{NaOAc}(16 \mathrm{mg}, 0.2$ mmol), in $1.0 \mathrm{~mL}$ of HFIP, following the general procedure, aniline $\mathbf{3 y}$ was obtained. Chromatographic purification (gradient elution: 5:95 $\rightarrow$ 30:70 $\mathrm{Et}_{2} \mathrm{O}$ - hexane) gave $3 \mathbf{y}$ (41 mg, 79\%), as a colourless oil.

Data for 3y: $\boldsymbol{R}_{f} 0.30\left(25 \% \mathrm{Et}_{2} \mathrm{O}\right.$ - hexane). ${ }^{1} \mathbf{H}$ NMR (300 $\left.\mathbf{M H z}, \mathbf{C D C l}_{3}\right) \delta$ 7.12-7.32 (5 H, m, $\mathrm{Ph}), 7.03(1 \mathrm{H}, \mathrm{d}, J=2.1 \mathrm{~Hz}, 3-\mathrm{H}), 6.78(1 \mathrm{H}, \mathrm{d}, J=2.0 \mathrm{~Hz}, 5-\mathrm{H}), 3.81\left(2 \mathrm{H}, \mathrm{br} \mathrm{s}, \mathrm{NH}_{2}\right), 2.13(3 \mathrm{H}, \mathrm{s}$, Me), 1.61 (6 H, s, 2 x Me). ${ }^{13}$ C NMR (75 MHz, CDCl $_{3}$ ) $\delta 150.8$ (1 C, C Ar), 141.2 (1 C, C Ar), 138.9 (1 C, C Ar), 128.1 (2 C, CH Ar), 127.7 (1 C, CH Ar), 126.8 (2 C, CH Ar), 125.7 (1 C, CH Ar), 125.3 (1 C, CH Ar), 123.3 (1 C, C Ar), 119.0 (1 C, C-6), 42.3 (1 C, C-7), 30.9 (2 C, 2 x Me), 18.4 (1 C, Me). HRMS (ESI): calculated for $\mathrm{C}_{16} \mathrm{H}_{19} \mathrm{CIN}[\mathrm{M}+\mathrm{H}]^{+}$requires $\mathrm{m} / \mathrm{z}$ 260.1201, found $\mathrm{m} / \mathrm{z}, 260.1194$.

\subsection{3-Fluoro-2-methyl-4-(2-phenylpropan-2-yl)aniline, $3 z$.}<smiles>Nc1cccc(F)c1N</smiles>

(1.0 equiv.)<smiles>C=C(C)Pc1ccccc1</smiles>

$2 a$

(2.0 equiv.)

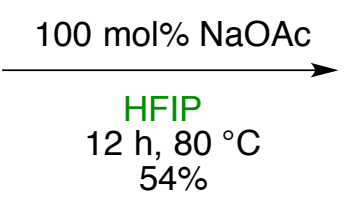

$54 \%$

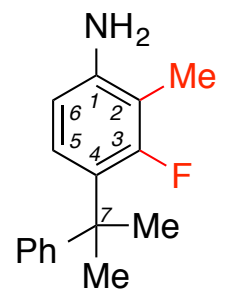

$3 z$

From aniline $\mathbf{1 z}(25 \mathrm{mg}, 0.2 \mathrm{mmol})$, alkene $\mathbf{2 a}(52 \mu \mathrm{l}, 0.4 \mathrm{mmol})$ and $\mathrm{NaOAc}(16 \mathrm{mg}, 0.2$ mmol), in $1.0 \mathrm{~mL}$ of HFIP, following the general procedure, aniline $\mathbf{3 z}$ was obtained. Chromatographic purification (gradient elution: 5:95 $\rightarrow$ 30:70 $\mathrm{Et}_{2} \mathrm{O}$ - hexane) gave $\mathbf{3 z}(26 \mathrm{mg}, 54 \%$ ), as a white foam and starting material $\mathbf{1 z}(8 \mathrm{mg}, 29 \%)$ recovered.

Data for 3z: $\boldsymbol{R}_{f} 0.35\left(25 \% \mathrm{Et}_{2} \mathrm{O}\right.$ - hexane). ${ }^{1} \mathbf{H}$ NMR (300 MHz, $\left.\mathbf{C D C l}_{3}\right) \delta$ 7.13-7.32 (5 H, m, $\mathrm{Ph}), 7.09\left(1 \mathrm{H}, \mathrm{dd}, J_{5-6}=8.5 \mathrm{~Hz}\right.$ and $\left.J_{5-F}=8.7 \mathrm{~Hz}, 5-\mathrm{H}\right), 6.48\left(1 \mathrm{H}, \mathrm{dd}, J_{6-5}=8.3 \mathrm{~Hz}\right.$ and $J_{6-F}=1.2 \mathrm{~Hz}$, 6-H), $3.68\left(2 \mathrm{H}\right.$, br s, $\left.\mathrm{NH}_{2}\right), 1.97\left(3 \mathrm{H}, \mathrm{d}, J_{M e-F}=2.0 \mathrm{~Hz}, \mathrm{Me}\right), 1.66\left(6 \mathrm{H}, \mathrm{d}, J_{M e-F}=0.8 \mathrm{~Hz}, 2 \mathrm{x} \mathrm{Me}\right)$. 
${ }^{13}$ C NMR (75 MHz, CDCl $) \delta 160.0\left(1 \mathrm{C}, \mathrm{d}, J_{3-F}=245.9 \mathrm{~Hz}, \mathrm{C}-3\right), 151.0(1 \mathrm{C}, \mathrm{C} \mathrm{Ph}), 144.7$ (1 C, d, $\left.J_{l-F}=7.8 \mathrm{~Hz}, \mathrm{C}-1\right), 128.0(2 \mathrm{C}, \mathrm{CH} \mathrm{Ph}), 127.0\left(1 \mathrm{C}, \mathrm{d}, J_{4-F}=13.7 \mathrm{~Hz}, \mathrm{C}-4\right), 125.8\left(2 \mathrm{C}, \mathrm{d}, J_{l-F}=1.3\right.$ $\mathrm{Hz}, \mathrm{CH} \mathrm{Ph}), 125.5$ (1 C, CH Ph), 124.8 (1 C, d, J J-F $=7.2 \mathrm{~Hz}, \mathrm{C}-5), 110.9$ (1 C, d, J2-F $=20.3 \mathrm{~Hz}, \mathrm{C}-$ 2), $109.6(1 \mathrm{C}, \mathrm{C}-6), 41.2$ (1 C, C-7), 30.0 (2 C, d, $\left.J_{M e-F}=2.2 \mathrm{~Hz}, 2 \times \mathrm{Me}\right), 8.8\left(1 \mathrm{C}, \mathrm{d}, J_{M e-F}=7.8 \mathrm{~Hz}\right.$, Me). HRMS (ESI): calculated for $\mathrm{C}_{16} \mathrm{H}_{19} \mathrm{FN}[\mathrm{M}+\mathrm{H}]^{+}$requires $m / z$ 244.1496, found $m / z$ 244.1490.

3.27. 4-(2-Phenylpropan-2-yl)naphthalen-1-amine, 3aa.

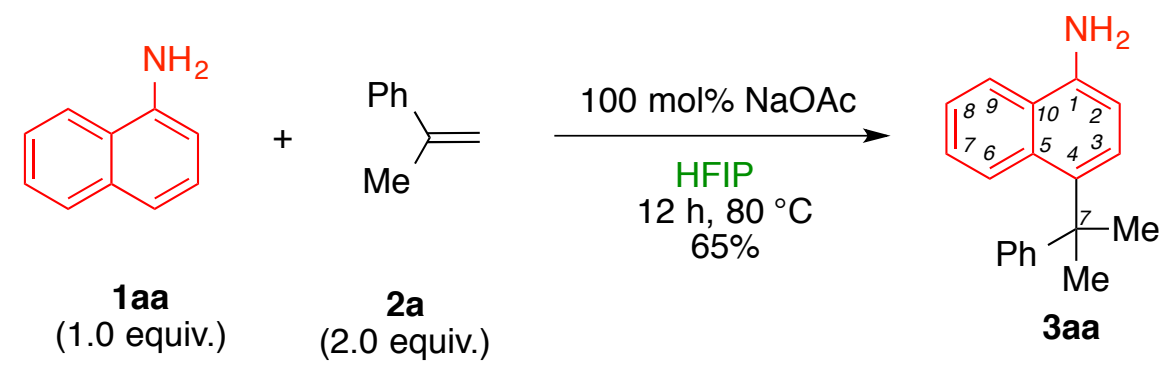

From aniline 1aa $(29 \mathrm{mg}, 0.2 \mathrm{mmol})$, alkene 2a $(52 \mu \mathrm{l}, 0.4 \mathrm{mmol})$ and NaOAc (16 mg, 0.2 $\mathrm{mmol}$ ), in $1.0 \mathrm{~mL}$ of HFIP, following the general procedure, aniline 3aa was obtained. Chromatographic purification (gradient elution: 5:95 $\rightarrow$ 30:70 $\mathrm{Et}_{2} \mathrm{O}$ - hexane) gave 3aa (34 mg, $65 \%)$, as a yellow oil and starting material $1 \mathbf{a a}(8 \mathrm{mg}, 28 \%)$ recovered.

Data for 3aa: $\boldsymbol{R}_{f} 0.40\left(25 \% \mathrm{Et}_{2} \mathrm{O}\right.$ - hexane). ${ }^{1} \mathbf{H}$ NMR (300 $\left.\mathbf{M H z}, \mathbf{C D C l}_{3}\right) \delta$ 7.77-7.83 (1 H, m, Ar), 7.66-7.74 (2 H, m, Ar), 7.17-7.45 (8 H, m, Ar), 3.57 (2 H, br s, $\left.\mathrm{NH}_{2}\right), 1.78$ (6 H, s, 2 x Me). ${ }^{13}$ C NMR (75 MHz, CDCl $) \delta 149.9$ (1 C, C Ar), 139.2 (1 C, C Ar), 133.4 (1 C, C Ar), 129.0 (2 C, CH Ar), 128.4 (1 C, CH Ar), 127.5 (1 C, C Ar), 126.4 (1 C, CH Ar), 126.0 (2 C, CH Ar), 125.4 (1 C, CH Ar), 125.3 (1 C, CH Ar), 125.0 (1 C, CH Ar), 124.6 (1 C, C Ar), 120.6 (1 C, CH Ar), 118.1 (1 C, C-2), 42.7 (1 C, C-7), 29.7 (2 C, 2 x Me). HRMS (ESI): calculated for $\mathrm{C}_{19} \mathrm{H}_{20} \mathrm{~N}[\mathrm{M}+\mathrm{H}]^{+}$requires $\mathrm{m} / \mathrm{z}$ 262.1591 , found $m / z 262.1590$.

3.28. $\quad N$-Methyl-4-(2-phenylbutan-2-yl)aniline, 3ab.

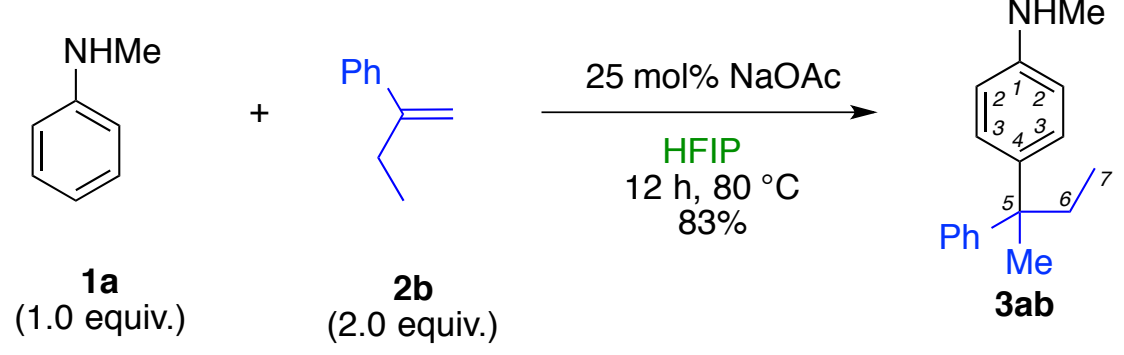

From aniline $1 \mathbf{a}(22 \mathrm{mg}, 0.2 \mathrm{mmol})$, alkene $\mathbf{2 b}(52 \mathrm{mg}, 0.4 \mathrm{mmol})$ and $\mathrm{NaOAc}(4.0 \mathrm{mg}, 0.05$ $\mathrm{mmol}$ ), in $1.0 \mathrm{~mL}$ of HFIP, following the general procedure, aniline 3ab was obtained. Chromatographic purification (gradient elution: 5:95 $\rightarrow$ 30:70 $\mathrm{Et}_{2} \mathrm{O}$ - hexane) gave 3ab (40 g, 83\%), as a yellow oil. 
Data for 3ab: $\boldsymbol{R}_{f} 0.40\left(25 \% \mathrm{Et}_{2} \mathrm{O}-\right.$ hexane). ${ }^{1} \mathbf{H} \mathbf{N M R}\left(300 \mathbf{~ M H z}, \mathbf{C D C l}_{3}\right) \delta 7.15-7.47(5 \mathrm{H}$, m, Ph), $7.05(2 \mathrm{H}, \mathrm{d}, J=8.7 \mathrm{~Hz}, 3-\mathrm{H}), 6.57(2 \mathrm{H}, \mathrm{d}, J=8.7 \mathrm{~Hz}, 2-\mathrm{H}), 3.56(1 \mathrm{H}, \mathrm{br} \mathrm{s}, \mathrm{NH}), 2.85(3 \mathrm{H}$, s, NMe), $2.13\left(2 \mathrm{H}, \mathrm{q}, J=7.3 \mathrm{~Hz}, 6-\mathrm{H}_{2}\right), 1.60(6 \mathrm{H}, \mathrm{s}, 2 \mathrm{x} \mathrm{Me}), 0.77\left(3 \mathrm{H}, \mathrm{t}, J=7.3 \mathrm{~Hz}, 7-\mathrm{H}_{3}\right) .{ }^{13} \mathbf{C}$ NMR (75 MHz, CDCl $) \delta 150.5$ (1 C, C Ar), 147.1 (1 C, C Ar), 138.6 (1 C, C Ar), 128.3 (2 C, CH Ar), 127.9 (2 C, CH Ar), 127.6 (2 C, CH Ar), 125.4 (1 C, CH Ph), 112.1 (2 C, C-2), 45.8 (1 C, C-5), 34.3 (1 C, Me), 31.0 (1 C, NMe), 27.2 (1 C, C-6), 9.4 (1 C, C-7). HRMS (ESI): calculated for $\mathrm{C}_{17} \mathrm{H}_{22} \mathrm{~N}$ $[\mathrm{M}+\mathrm{H}]^{+}$requires $\mathrm{m} / \mathrm{z}, 240.1746$, found $\mathrm{m} / \mathrm{z}, 240.1752$.

3.29. $\quad N$-Methyl-4-(3-methyl-2-phenylbutan-2-yl)aniline, 3ac.

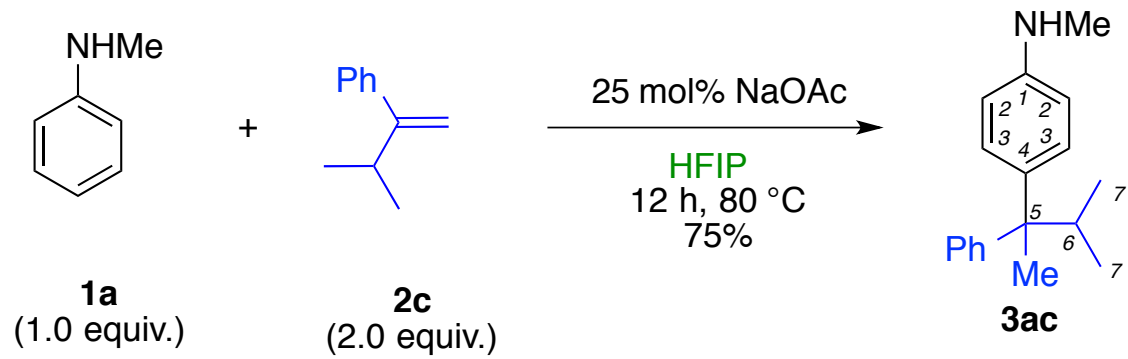

From aniline 1a (22 mg, $0.2 \mathrm{mmol})$, alkene $2 \mathrm{c}(60 \mathrm{mg}, 0.4 \mathrm{mmol})$ and $\mathrm{NaOAc}(4.0 \mathrm{mg}, 0.05$ $\mathrm{mmol}$ ), in $1.0 \mathrm{~mL}$ of HFIP, following the general procedure, aniline 3ac was obtained. Chromatographic purification (gradient elution: 5:95 $\rightarrow$ 30:70 $\mathrm{Et}_{2} \mathrm{O}$ - hexane) gave 3ac (38 mg, $75 \%$ ), as a yellow oil.

Data for 3ac: $\boldsymbol{R}_{f} 0.40$ (25\% $\mathrm{Et}_{2} \mathrm{O}$ - hexane). ${ }^{1} \mathbf{H}$ NMR (300 $\left.\mathbf{M H z}, \mathbf{C D C l}_{3}\right) \delta$ 7.13-7.44 (5 H, m, Ph), $7.08(2 \mathrm{H}, \mathrm{d}, J=8.7 \mathrm{~Hz}, 3-\mathrm{H}), 6.54(2 \mathrm{H}, \mathrm{d}, J=8.7 \mathrm{~Hz}, 2-\mathrm{H}), 3.54(1 \mathrm{H}, \mathrm{br} \mathrm{s}, \mathrm{NH}), 2.82(3 \mathrm{H}$, s, NMe), $2.67(2 \mathrm{H}$, sept, $J=6.7 \mathrm{~Hz}, 6-\mathrm{H}), 1.58(3 \mathrm{H}, \mathrm{s}, \mathrm{Me}), 0.87\left(3 \mathrm{H}, \mathrm{d}, J=6.7 \mathrm{~Hz}, 7-\mathrm{H}_{3}\right), 0.83(3$ $\left.\mathrm{H}, \mathrm{d}, J=6.7 \mathrm{~Hz}, 7-\mathrm{H}_{3}\right) .{ }^{13} \mathbf{C} \mathbf{N M R}\left(\mathbf{7 5} \mathbf{M H z}, \mathbf{C D C l}_{3}\right) \delta 149.5$ (1 C, C Ar), 146.9 (1 C, C Ar), 137.5 (1 C, C Ar), 128.8 (2 C, CH Ar), 128.0 (2 C, CH Ar), 127.7 (2 C, CH Ar), 125.2 (1 C, CH Ar), 111.9 (2 C, C-2), 48.9 (1 C, C-5), 34.4 (1 C, Me), 31.0 (1 C, NMe), 27.8 (1 C, C-6), 19.0 (1 C, C-7), 18.9 (1 C, C-7). HRMS (ESI): calculated for $\mathrm{C}_{18} \mathrm{H}_{24} \mathrm{~N}[\mathrm{M}+\mathrm{H}]^{+}$requires $m / z$ 254.1903, found $\mathrm{m} / z$ 254.1911.

\subsection{4-(1-Cyclohexyl-1-phenylethyl)- $N$-methylaniline, 3ad.}<smiles>CNc1ccccc1</smiles>

$1 \mathbf{a}$ (1.0 equiv.)

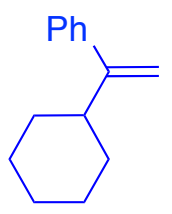

2d

(2.0 equiv.)

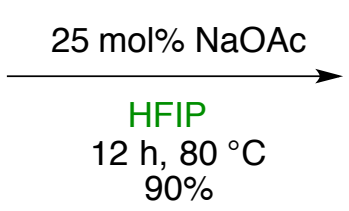

$90 \%$

From aniline 1a (22 mg, $0.2 \mathrm{mmol})$, alkene $2 \mathbf{d}(76 \mathrm{mg}, 0.4 \mathrm{mmol})$ and $\mathrm{NaOAc}(4.0 \mathrm{mg}, 0.05$ $\mathrm{mmol}$ ), in $1.0 \mathrm{~mL}$ of HFIP, following the general procedure, aniline 3ad was obtained. 
Chromatographic purification (gradient elution: 5:95 $\rightarrow$ 30:70 $\mathrm{Et}_{2} \mathrm{O}$ - hexane) gave 3ad (53 mg, 90\%), as a colourless oil.

Data for 3ad: $\boldsymbol{R}_{f} 0.40\left(50 \% \mathrm{Et}_{2} \mathrm{O}\right.$ - hexane). ${ }^{1} \mathbf{H}$ NMR (300 $\left.\mathbf{M H z}, \mathbf{C D C l}_{3}\right) \delta 7.12-7.40(5 \mathrm{H}$, m, Ph), $7.07(2 \mathrm{H}, \mathrm{d}, J=8.7 \mathrm{~Hz}, 3-\mathrm{H}), 6.53(2 \mathrm{H}, \mathrm{d}, J=8.6 \mathrm{~Hz}, 2-\mathrm{H}), 3.58(1 \mathrm{H}, \mathrm{br} \mathrm{s}, \mathrm{NH}), 2.81(3 \mathrm{H}$, s, NMe), $2.19(1 \mathrm{H}, \mathrm{t}, J=11.5 \mathrm{~Hz}, \mathrm{CH} c-\mathrm{Hex}), 1.64-1.81(5 \mathrm{H}, \mathrm{d}, J=11.5 \mathrm{~Hz}, \mathrm{CH} c-\mathrm{Hex}), 1.60(3 \mathrm{H}$, s, Me), 0.79-1.42 (5 H, m, CH c-Hex). $\left.{ }^{13} \mathbf{C ~ N M R ~ ( 7 5 ~ M H z , ~} \mathbf{C D C l}_{3}\right) \delta 149.4$ (1 C, C Ar), 146.9 (1 C, C Ar), 137.4 (1 C, C Ar), 128.7 (2 C, CH Ar), 127.9 (2 C, CH Ar), 127.7 (2 C, CH Ar), 125.2 (1 C, $\mathrm{CH} \mathrm{Ph}), 111.9$ (2 C, C-2), 48.8 (1 C, C-5), 45.8 (1 C, C-6), 31.0 (1 C, NMe), 29.03 (1 C, $\mathrm{CH}_{2} c-\mathrm{Hex}$ ), 28.96 (1 C, $\left.\mathrm{CH}_{2} c-\mathrm{Hex}\right), 27.44$ (1 C, $\mathrm{CH}_{2} c$-Hex), 27.42 (1 C, $\left.\mathrm{CH}_{2} c-\mathrm{Hex}\right), 27.0\left(1 \mathrm{C}, \mathrm{CH}_{2} c-\mathrm{Hex}\right)$, 23.7 (1 C, Me). HRMS (ESI): calculated for $\mathrm{C}_{21} \mathrm{H}_{28} \mathrm{~N}[\mathrm{M}+\mathrm{H}]^{+}$requires $\mathrm{m} / \mathrm{z}$ 294.2216, found $\mathrm{m} / \mathrm{z}$ 294.2228.

3.31. 4-[2-(4-Iodophenyl)propan-2-yl]- $N$-methylaniline, 3ae.

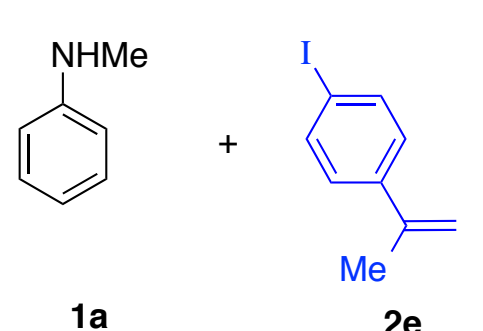

(1.0 equiv.)

(2.0 equiv.)

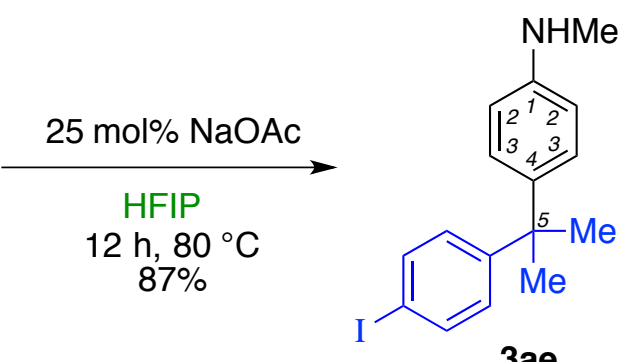

3ae

From aniline 1a (22 mg, $0.2 \mathrm{mmol})$, alkene $2 \mathbf{e}(96 \mathrm{mg}, 0.4 \mathrm{mmol})$ and $\mathrm{NaOAc}(4.0 \mathrm{mg}, 0.05$ $\mathrm{mmol}$ ), in $1.0 \mathrm{~mL}$ of HFIP, following the general procedure, aniline 3ae was obtained. Chromatographic purification (gradient elution: 5:95 $\rightarrow$ 30:70 $\mathrm{Et}_{2} \mathrm{O}$ - hexane) gave 3ae (61 mg, $87 \%$ ), as a colourless oil.

Data for 3ae: $\boldsymbol{R}_{f} 0.40\left(25 \% \mathrm{Et}_{2} \mathrm{O}\right.$ - hexane). ${ }^{1} \mathbf{H} \mathbf{N M R}\left(\mathbf{3 0 0} \mathbf{M H z}, \mathbf{C D C l}_{3}\right) \delta 7.56(2 \mathrm{H}, \mathrm{d}, J=$ 8.7 Hz, 3-H), $7.03(2 \mathrm{H}, \mathrm{d}, J=8.7 \mathrm{~Hz}, \mathrm{CH} \mathrm{Ar}), 7.00(2 \mathrm{H}, \mathrm{d}, J=8.7 \mathrm{~Hz}, \mathrm{CH} \mathrm{Ar}), 6.54(2 \mathrm{H}, \mathrm{d}, J=8.7$ $\mathrm{Hz}, 2-\mathrm{H}), 3.63$ (1 H, br s, NH), 2.83 (3 H, s, NMe), 1.62 (6 H, s, 2 x Me). ${ }^{13} \mathbf{C ~ N M R}(75 \text { MHz, CDCl})_{3}$ $\delta 151.4$ (1 C, C Ar), 147.4 (1 C, C Ar), 138.9 (1 C, C Ar), 137.1 (2 C, CH Ar), 129.2 (2 C, CH Ar), 127.6 (2 C, CH Ar), 112.2 (2 C, C-2), 90.9 (1 C, C Ar), 42.2 (1 C, C-5), 31.0 (1 C, NMe), 30.9 (2 C, $2 \times \mathrm{Me}$ ). HRMS (ESI): calculated for $\mathrm{C}_{16} \mathrm{H}_{19} \mathrm{NI}[\mathrm{M}+\mathrm{H}]^{+}$requires $\mathrm{m} / z$ 352.0556, found $\mathrm{m} / \mathrm{z} 352.0553$. 
3.32. $N$-Methyl-4-[2-(thiophen-2-yl)propan-2-yl]aniline, 3af.

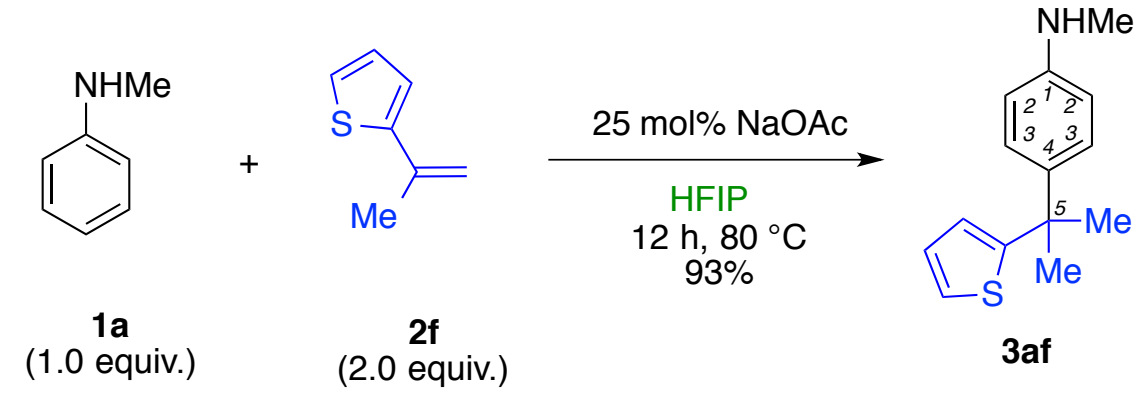

From aniline 1a (22 mg, $0.2 \mathrm{mmol})$, alkene $2 \mathbf{f}(52 \mathrm{mg}, 0.4 \mathrm{mmol})$ and $\mathrm{NaOAc}(4.0 \mathrm{mg}, 0.05$ mmol), in $1.0 \mathrm{~mL}$ of HFIP, following the general procedure, aniline 3af was obtained. Chromatographic purification (gradient elution: 5:95 $\rightarrow$ 30:70 $\mathrm{Et}_{2} \mathrm{O}$ - hexane) gave 3af (43 mg, 93\%), as a red oil.

Data for 3af: $\boldsymbol{R}_{f} 0.35\left(25 \% \mathrm{Et}_{2} \mathrm{O}\right.$ - hexane). ${ }^{1} \mathbf{H} \mathbf{N M R}\left(\mathbf{3 0 0} \mathbf{M H z}, \mathbf{C D C l}_{3}\right) \delta 7.19(2 \mathrm{H}, \mathrm{d}, J=$ 8.7 Hz, 3-H), $7.15(1 \mathrm{H}, \mathrm{dd}, J=5.1$ and $1.2 \mathrm{~Hz}, \mathrm{CH}$ Thioph), $6.93(1 \mathrm{H}, \mathrm{dd}, J=5.1$ and $3.5 \mathrm{~Hz}, \mathrm{CH}$ Thioph), 6.84 (1 H, dd, $J=3.5$ and $1.2 \mathrm{~Hz}, \mathrm{CH}$ Thioph), $6.58(2 \mathrm{H}, \mathrm{d}, J=8.7 \mathrm{~Hz}, 2-\mathrm{H}), 3.59$ (1 H, br s, NH), 2.85 (3 H, s, NMe), 1.78 (6 H, s, 2 x Me). ${ }^{13} \mathbf{C}$ NMR (75 MHz, CDCl $) \delta 157.6$ (1 C, C-1), 147.6 (1 C, C-4), 138.7 (1 C, C Thioph), 127.1 (2 C, C-3), 126.3 (1 C, CH Thioph), 123.2 (1 C, CH Thioph), 123.0 (1 C, CH Thioph), 112.1 (2 C, C-2), 40.9 (1 C, C-5), 32.3 (2 C, 2 x Me), 30.9 (1 C, $\mathrm{NMe}$ ). HRMS (ESI): calculated for $\mathrm{C}_{14} \mathrm{H}_{18} \mathrm{NS}[\mathrm{M}+\mathrm{H}]^{+}$requires $\mathrm{m} / \mathrm{z}, 232.1154$, found $\mathrm{m} / \mathrm{z} 232.1162$.

\subsection{4-(1,1-Diphenylethyl)- $N$-methylaniline, 3ag.}

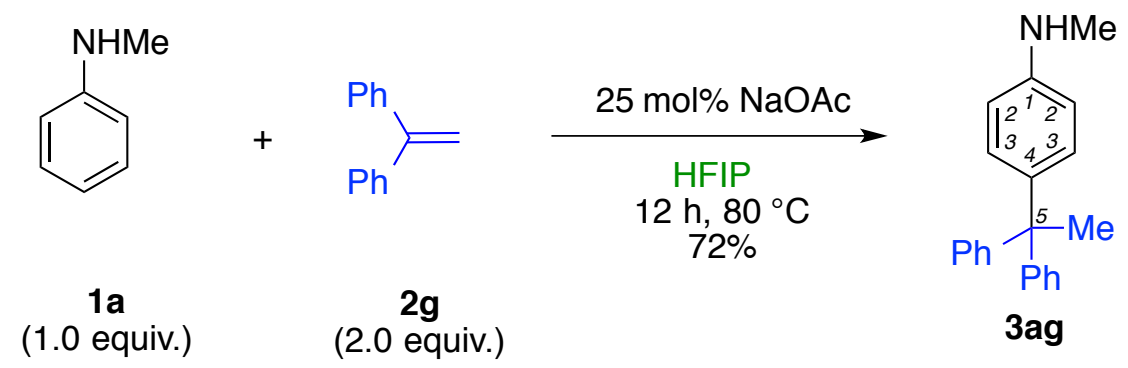

From aniline 1a (22 mg, $0.2 \mathrm{mmol})$, alkene $2 \mathbf{g}(72 \mathrm{mg}, 0.4 \mathrm{mmol})$ and $\mathrm{NaOAc}(4.0 \mathrm{mg}, 0.05$ mmol), in $1.0 \mathrm{~mL}$ of HFIP, following the general procedure, aniline 3ag was obtained. Chromatographic purification (gradient elution: 5:95 $\rightarrow$ 50:50 $\mathrm{Et}_{2} \mathrm{O}$ - hexane) gave 3ag (41 mg, $72 \%)$, as a yellow oil.

Data for 3ag: $\boldsymbol{R}_{f} 0.30\left(30 \% \mathrm{Et}_{2} \mathrm{O}\right.$ - hexane). ${ }^{1} \mathbf{H}$ NMR (300 MHz, $\left.\mathbf{C D C l}_{3}\right) \delta 7.04-7.36(10 \mathrm{H}$, m, Ph), $6.91(2 \mathrm{H}, \mathrm{d}, J=8.7 \mathrm{~Hz}, 3-\mathrm{H}), 6.54(2 \mathrm{H}, \mathrm{d}, J=8.7 \mathrm{~Hz}, 2-\mathrm{H}), 3.69(1 \mathrm{H}, \mathrm{br} \mathrm{s}, \mathrm{NH}), 2.83(3 \mathrm{H}$, s, NMe), 2.15 (3 H, s, Me). ${ }^{13}$ C NMR (75 MHz, CDCl $) \delta 149.9$ (2 C, C Ar), 147.4 (1 C, C Ar), 138.0 (1 C, C Ar), 129.7 (2 C, C-3), 128.8 (4 C, CH Ar), 127.9 (4 C, CH Ar), 125.9 (2 C, CH Ph), 112.0 (2 C, C-2), 52.9 (1 C, C-5), 31.0 (1 C, NMe), 30.7 (1 C, Me). HRMS (ESI): calculated for $\mathrm{C}_{21} \mathrm{H}_{22} \mathrm{~N}$ 
$[\mathrm{M}+\mathrm{H}]^{+}$requires $\mathrm{m} / \mathrm{z}, 288.1746$, found $\mathrm{m} / \mathrm{z} 288.1748$.

3.34. $N$-Methyl-4-(1-methyl-1,2,3,4-tetrahydronaphthalen-1-yl)aniline, 3ah.<smiles>CNc1ccccc1</smiles>

$1 \mathrm{a}$ (1.0 equiv.)

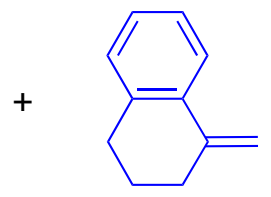

$2 h$

(2.0 equiv.)

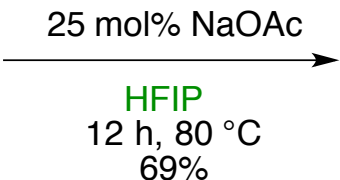

$69 \%$

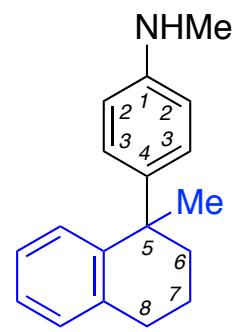

3ah

From aniline $\mathbf{1 a}(22 \mathrm{mg}, 0.2 \mathrm{mmol})$, alkene $\mathbf{2 h}(60 \mathrm{mg}, 0.4 \mathrm{mmol})$ and $\mathrm{NaOAc}(4.0 \mathrm{mg}, 0.05$ mmol), in $1.0 \mathrm{~mL}$ of HFIP, following the general procedure, aniline 3ah was obtained. Chromatographic purification (gradient elution: 5:95 $\rightarrow$ 30:70 $\mathrm{Et}_{2} \mathrm{O}$ - hexane) gave 3ah (31 mg, $69 \%$ ), as a yellow oil.

Data for 3ah: $\boldsymbol{R}_{f} 0.35$ (25\% Et ${ }_{2} \mathrm{O}$ - hexane). ${ }^{1} \mathbf{H}$ NMR (300 MHz, $\left.\mathbf{C D C l}_{3}\right) \delta$ 7.03-7.14 (5 H, m, Ph), $6.95(2 \mathrm{H}, \mathrm{d}, J=8.6 \mathrm{~Hz}, 3-\mathrm{H}), 6.52(2 \mathrm{H}, \mathrm{d}, J=8.6 \mathrm{~Hz}, 2-\mathrm{H}), 3.58(1 \mathrm{H}, \mathrm{br} \mathrm{s}, \mathrm{NH}), 2.84(2 \mathrm{H}$, t, $\left.J=6.8 \mathrm{~Hz}, 8-\mathrm{H}_{2}\right), 2.82(3 \mathrm{H}, \mathrm{s}, \mathrm{NMe}), 2.03(1 \mathrm{H}, \mathrm{ddd}, J=13.1,7.9$ and $3.4 \mathrm{~Hz}, 6-\mathrm{Ha}), 1.75-1.93(1$ $\mathrm{H}, \mathrm{m}, 6-\mathrm{Hb}), 1.69-1.82\left(2 \mathrm{H}, \mathrm{m}, 7-\mathrm{H}_{2}\right), 1.69(3 \mathrm{H}, \mathrm{s}, \mathrm{Me}) .{ }^{13} \mathbf{C}$ NMR (75 MHz, CDCl $) \delta 147.1(1 \mathrm{C}$, C Ar), 145.1 (1 C, C Ar), 140.7 (1 C, C Ar), 137.2 (1 C, C Ar), 129.3 (1 C, CH Ar), 129.0 (1 C, CH Ar), 128.3 (2 C, C-3), 125.8 (1 C, CH Ar), 125.6 (1 C, CH Ar), 112.1 (2 C, C-2), 42.2 (1 C, C-5), 41.6 (1 C, C-6), 31.0 (1 C, NMe), 30.5 (1 C, C-8), 30.3 (1 C, Me), 19.8 (1 C, C-7). HRMS (ESI): calculated for $\mathrm{C}_{18} \mathrm{H}_{22} \mathrm{~N}[\mathrm{M}+\mathrm{H}]^{+}$requires $\mathrm{m} / z, 252.1746$, found $\mathrm{m} / z, 252.1756$.

\subsection{4-[1-(4-Methoxyphenyl)propyl]- $N$-methylaniline, 3ai.}
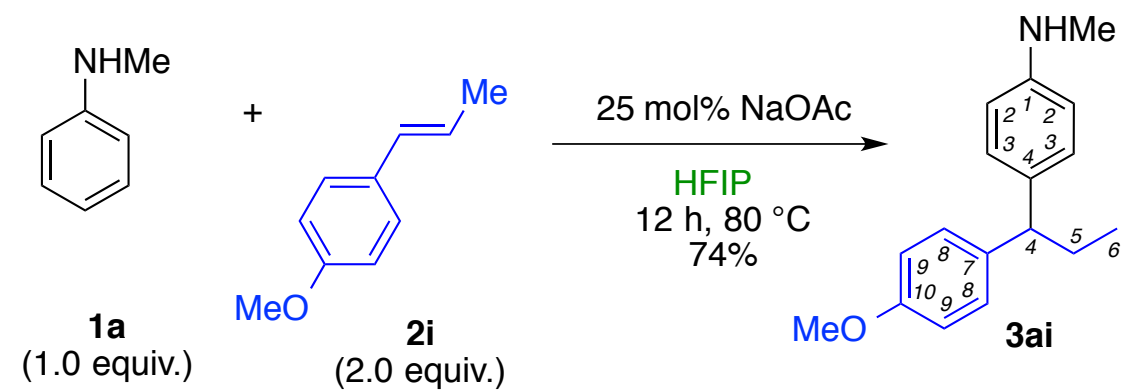

From aniline 1a (22 mg, $0.2 \mathrm{mmol})$, alkene $2 \mathbf{i}(60 \mathrm{mg}, 0.4 \mathrm{mmol})$ and $\mathrm{NaOAc}(4.0 \mathrm{mg}, 0.05$ $\mathrm{mmol}$ ), in $1.0 \mathrm{~mL}$ of HFIP, following the general procedure, aniline 3ai was obtained. Chromatographic purification (gradient elution: 5:95 $\rightarrow$ 30:70 $\mathrm{Et}_{2} \mathrm{O}$ - hexane) gave 3ai (39 mg, 74\%), as a yellow oil.

Data for 3ai: $\boldsymbol{R}_{f} 0.30\left(25 \% \mathrm{Et}_{2} \mathrm{O}\right.$ - hexane). ${ }^{1} \mathbf{H}$ NMR (300 MHz, $\left.\mathbf{C D C l}_{3}\right) \delta 7.14(2 \mathrm{H}, \mathrm{d}, J=$ $8.6 \mathrm{~Hz}, 3-\mathrm{H}), 7.05(2 \mathrm{H}, \mathrm{d}, J=8.4 \mathrm{~Hz}, 8-\mathrm{H}), 6.83(2 \mathrm{H}, \mathrm{d}, J=8.4 \mathrm{~Hz}, 9-\mathrm{H}), 6.56(2 \mathrm{H}, \mathrm{d}, J=8.6 \mathrm{~Hz}$, 
2-H), 3.77 (3 H, s, OMe), $3.65(1 \mathrm{H}, \mathrm{t}, J=7.8 \mathrm{~Hz}, 4-\mathrm{H}), 2.81(3 \mathrm{H}, \mathrm{s}, \mathrm{NMe}), 2.00$ (2 H, quint, $J=7.4$

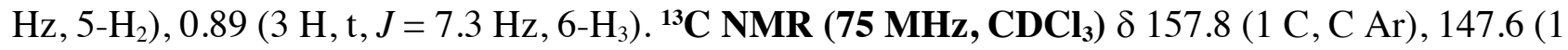
C, C Ar), 138.4 (1 C, C Ar), 134.6 (1 C, C Ar), 128.8 (2 C, CH Ar), 128.7 (2 C, CH Ar), 113.8 (2 C, C-9), 112.6 (2 C, C-2), 55.3 (1 C, OMe), 51.6 (1 C, C-4), 31.1 (1 C, NMe), 29.1 (1 C, C-5), 13.0 (1 C, C-6). HRMS (ESI): calculated for $\mathrm{C}_{17} \mathrm{H}_{22} \mathrm{NO}[\mathrm{M}+\mathrm{H}]^{+}$requires $m / z$ 256.1696, found $m / z$ 256.1698.

3.36. 2-[(2,3-Dimethylphenyl)amino]-5-(2-phenylpropan-2-yl)benzoic acid, 3aj.<smiles>C=C(C)Pc1ccccc1</smiles>

Mefenamic acid (1.0 equiv.)

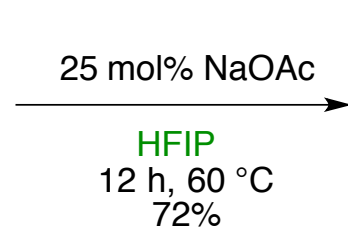

$2 a$ (2.0 equiv.)

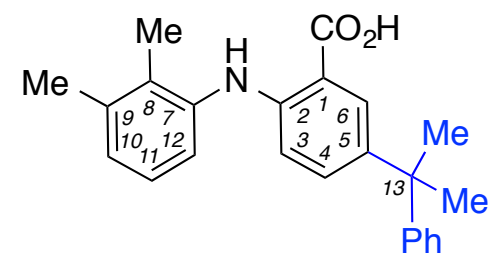

3aj

From Mefenamic acid (48 mg, $0.2 \mathrm{mmol})$, alkene $2 \mathbf{a}(52 \mu \mathrm{l}, 0.4 \mathrm{mmol})$ and $\mathrm{NaOAc}(4.0 \mathrm{mg}$, $0.05 \mathrm{mmol}$ ), in $1.0 \mathrm{~mL}$ of HFIP, following the general procedure, aniline 3aj was obtained. Chromatographic purification (gradient elution: $1: 99 \rightarrow 10: 90 \mathrm{MeOH}-\mathrm{CH}_{2} \mathrm{Cl}_{2}$ ) gave 3aj (52 mg, $72 \%)$, as a yellow foam.

Data for 3aj: $\boldsymbol{R}_{f} 0.40\left(5 \% \mathrm{MeOH}-\mathrm{CH}_{2} \mathrm{Cl}_{2}\right) .{ }^{1} \mathbf{H}$ NMR (300 MHz, $\left.\mathbf{C D C l}_{3}\right) \delta 12.02(1 \mathrm{H}$, br s, $\left.\mathrm{CO}_{2} \mathrm{H}\right), 9.04(1 \mathrm{H}$, br s, NH), $8.03(1 \mathrm{H}, \mathrm{d}, J=2.4 \mathrm{~Hz}, 6-\mathrm{H}), 7.23-7.30(4 \mathrm{H}, \mathrm{m}, \mathrm{Ph}), 7.13-7.20(2 \mathrm{H}$, $\mathrm{m}, \mathrm{CH} \mathrm{Ph}$ and 10-H), $7.09(1 \mathrm{H}, \mathrm{t}, J=7.5 \mathrm{~Hz}, 11-\mathrm{H}), 7.07(1 \mathrm{H}, \mathrm{dd}, J=9.0$ and $2.5 \mathrm{~Hz}, 4-\mathrm{H}), 7.02$ (1 $\mathrm{H}, \mathrm{t}, J=7.0 \mathrm{~Hz}, 12-\mathrm{H}), 6.68(1 \mathrm{H}, \mathrm{d}, J=9.0 \mathrm{~Hz}, 3-\mathrm{H}), 2.33(3 \mathrm{H}, \mathrm{s}, \mathrm{Me}), 2.20(3 \mathrm{H}, \mathrm{s}, \mathrm{Me}), 1.68(6$ $\mathrm{H}, \mathrm{s}, 2 \mathrm{x} \mathrm{Me}) .{ }^{13} \mathbf{C}$ NMR (75 MHz, $\left.\mathbf{C D C l}_{3}\right) \delta 174.3\left(1 \mathrm{C}, \mathrm{CO}_{2} \mathrm{H}\right), 150.6$ (1 C, C Ar), 148.5 (1 C, C Ar), 138.8 (1 C, C Ar), 138.6 (1 C, C Ar), 138.3 (1 C, C Ar), 135.1 (1 C, C-4), 132.6 (1 C, C Ar), 129.2 (1 C, C-6), 128.2 (2 C, CH Ph), 127.0 (1 C, C-12), 126.8 (2 C, CH Ph), 126.1 (1 C, C-10), 125.8 (1 C, CH Ph), 123.3 (1 C, C-11), 114.2 (1 C, C-3), 108.8 (1 C, C Ar), 42.2 (1 C, C-13), 30.8 (2 C, $2 \times \mathrm{Me}), 20.7$ (1 C, Me), 14.2 (1 C, Me). HRMS (ESI): calculated for $\mathrm{C}_{24} \mathrm{H}_{26} \mathrm{NO}_{2}[\mathrm{M}+\mathrm{H}]^{+}$requires $\mathrm{m} / \mathrm{z} 360.1958$, found $\mathrm{m} / \mathrm{z} 360.1951$.

3.37. 5-(1-Cyclohexyl-1-phenylethyl)-2-[(2,3-dimethylphenyl)amino]benzoic acid, 3ak.<smiles>Cc1cccc(Nc2ccccc2C(=O)O)c1C</smiles>

Mefenamic acid (1.0 equiv.)

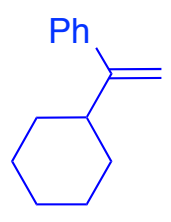

2d (2.0 equiv.)

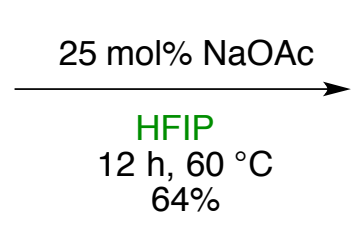

$64 \%$

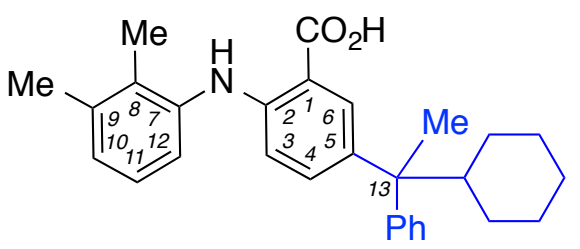

3ak 
From Mefenamic acid (48 mg, $0.2 \mathrm{mmol})$, alkene $2 d$ (76 mg, $0.4 \mathrm{mmol})$ and $\mathrm{NaOAc}(4.0 \mathrm{mg}$, $0.05 \mathrm{mmol}$ ), in $1.0 \mathrm{~mL}$ of HFIP, following the general procedure, aniline 3ak was obtained. Chromatographic purification (gradient elution: 1:99 $\rightarrow$ 10:90 $\mathrm{MeOH}-\mathrm{CH}_{2} \mathrm{Cl}_{2}$ ) gave 3ak (54 mg, $64 \%$ ), as a yellow foam.

Data for 3ak: $\boldsymbol{R}_{f} 0.40\left(5 \% \mathrm{MeOH}-\mathrm{CH}_{2} \mathrm{Cl}_{2}\right)$. ${ }^{1} \mathbf{H}$ NMR (300 MHz, $\left.\mathbf{C D C l}_{3}\right) \delta 11.78(1 \mathrm{H}$, br s, $\left.\mathrm{CO}_{2} \mathrm{H}\right), 9.03(1 \mathrm{H}$, br s, NH), $8.01(1 \mathrm{H}, \mathrm{d}, J=2.3 \mathrm{~Hz}, 6-\mathrm{H}), 7.23-7.30(4 \mathrm{H}, \mathrm{m}, \mathrm{Ph}), 7.17(1 \mathrm{H}, \mathrm{d}, J=$ $7.8 \mathrm{~Hz}, 10-\mathrm{H}), 7.13-7.17(1 \mathrm{H}, \mathrm{m}, \mathrm{CH} \mathrm{Ph}), 7.12(1 \mathrm{H}, \mathrm{dd}, J=8.8$ and $2.5 \mathrm{~Hz}, 4-\mathrm{H}), 7.10(1 \mathrm{H}, \mathrm{t}, J=$ $7.5 \mathrm{~Hz}, 11-\mathrm{H}), 7.03(1 \mathrm{H}, \mathrm{d}, J=7.2 \mathrm{~Hz}, 12-\mathrm{H}), 6.68(1 \mathrm{H}, \mathrm{d}, J=9.0 \mathrm{~Hz}, 3-\mathrm{H}), 2.35(3 \mathrm{H}, \mathrm{s}, \mathrm{Me}), 2.21$ $(3 \mathrm{H}, \mathrm{s}, \mathrm{Me}), 2.22(1 \mathrm{H}, \mathrm{t}, J=11.1 \mathrm{~Hz}, \mathrm{CH} c-\mathrm{Hex}), 1.57-1.85(5 \mathrm{H}, \mathrm{m}, \mathrm{CH} c-\mathrm{Hex}), 1.63(3 \mathrm{H}, \mathrm{s}, \mathrm{Me})$ 0.82-1. 47 (5 H, m, CH c-Hex). ${ }^{13} \mathbf{C}$ NMR (75 MHz, $\left.\mathbf{C D C l}_{3}\right) \delta 174.4\left(1 \mathrm{C}, \mathrm{CO}_{2} \mathrm{H}\right), 148.6(1 \mathrm{C}, \mathrm{C} \mathrm{Ar})$, 148.2 (1 C, C Ar), 138.9 (1 C, C Ar), 138.3 (1 C, C Ar), 136.3 (1 C, C-4), 135.8 (1 C, C Ar), 132.6 (1 C, C Ar), 130.7 (1 C, C-6), 127.9 (2 C, CH Ph), 127.8 (2 C, CH Ph), 127.0 (1 C, C-12), 126.0 (1 C, C-10), 125.5 (1 C, CH Ph), 123.4 (1 C, C-11), 113.8 (1 C, C-3), 108.8 (1 C, C Ar), 48.7 (1 C, C13), 45.7 (1 C, $\mathrm{CH} c$-Hex), 29.0 (1 C, $\mathrm{CH}_{2} c$-Hex), 28.9 (1 C, $\left.\mathrm{CH}_{2} c-\mathrm{Hex}\right), 27.39$ (1 C, $\left.\mathrm{CH}_{2} c-\mathrm{Hex}\right)$, 27.36 (1 C, $\mathrm{CH}_{2} c$-Hex), 26.9 (1 C, $\mathrm{CH}_{2} c$-Hex), 23.4 (1 C, Me), 20.7 (1 C, Me), 14.2 (1 C, Me). HRMS (ESI): calculated for $\mathrm{C}_{29} \mathrm{H}_{34} \mathrm{NO}_{2}[\mathrm{M}+\mathrm{H}]^{+}$requires $m / z$ 428.2584, found $m / z$, 428.2579.

3.38. 2-[(2,3-Dimethylphenyl)amino]-5-[2-(4-iodophenyl)propan-2-yl]benzoic acid, 3al.<smiles>Cc1cccc(Nc2ccccc2C(=O)O)c1C</smiles>

Mefenamic acid (1.0 equiv.)

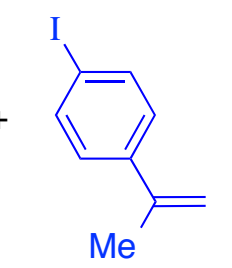

(2.0 equiv.)

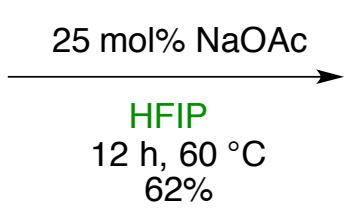
$62 \%$

From Mefenamic acid (48 mg, $0.2 \mathrm{mmol})$, alkene $2 \mathbf{e}(96 \mathrm{mg}, 0.4 \mathrm{mmol})$ and $\mathrm{NaOAc}(4.0 \mathrm{mg}$, $0.05 \mathrm{mmol}$ ), in $1.0 \mathrm{~mL}$ of HFIP, following the general procedure, aniline 3al was obtained. Chromatographic purification (gradient elution: $\left.1: 99 \rightarrow 10: 90 \mathrm{MeOH}-\mathrm{CH}_{2} \mathrm{Cl}_{2}\right)$ gave 3al $(60 \mathrm{mg}$, $62 \%$ ), as a white foam.

Data for 3al: $\boldsymbol{R}_{f} 0.40\left(5 \% \mathrm{MeOH}-\mathrm{CH}_{2} \mathrm{Cl}_{2}\right)$. ${ }^{1} \mathbf{H}$ NMR (300 MHz, $\left.\mathbf{C D C l}_{3}\right) \delta 11.02(1 \mathrm{H}$, br s, $\left.\mathrm{CO}_{2} \mathrm{H}\right), 9.04(1 \mathrm{H}$, br s, NH), $7.97(1 \mathrm{H}, \mathrm{d}, J=2.5 \mathrm{~Hz}, 6-\mathrm{H}), 7.57(2 \mathrm{H}, \mathrm{d}, J=8.5 \mathrm{~Hz}, 16-\mathrm{H}), 7.14(1$ $\mathrm{H}, \mathrm{dd}, J=7.8$ and $1.9 \mathrm{~Hz}, 10-\mathrm{H}), 7.09(1 \mathrm{H}, \mathrm{t}, J=7.5 \mathrm{~Hz}, 11-\mathrm{H}), 7.00-7.05(1 \mathrm{H}, \mathrm{m}, 12-\mathrm{H}), 7.03$ (1 $\mathrm{H}, \mathrm{dd}, J=9.0$ and $2.5 \mathrm{~Hz}, 4-\mathrm{H}), 6.99(2 \mathrm{H}, \mathrm{d}, J=8.7 \mathrm{~Hz}, 15-\mathrm{H}), 6.66(1 \mathrm{H}, \mathrm{d}, J=9.0 \mathrm{~Hz}, 3-\mathrm{H}), 2.32$ (3 H, s, Me), 2.18 (3 H, s, Me), 1.63 (6 H, s, 2 x Me). $\left.{ }^{13} \mathbf{C ~ N M R ~ ( 7 5 ~ M H z , ~ C D C l} 3\right) \delta 173.2(1 \mathrm{C}$, $\mathrm{CO}_{2} \mathrm{H}$ ), 150.5 (1 C, C Ar), 148.7 (1 C, C Ar), 138.7 (1 C, C Ar), 138.4 (1 C, C Ar), 137.7 (1 C, C Ar), 137.2 (2 C, C-16), 134.8 (1 C, C-4), 132.7 (1 C, C Ar), 129.2 (1 C, C-6), 129.1 (2 C, C-15), 127.1 (1 
C, C-12), 126.1 (1 C, C-10), 123.4 (1 C, C-11), 114.2 (1 C, C-3), 108.7 (1 C, C Ar), 91.2 (1 C, C-17), 42.2 (1 C, C-13), 30.6 (2 C, 2 x Me), 20.7 (1 C, Me), 14.2 (1 C, Me). HRMS (ESI): calculated for $\mathrm{C}_{24} \mathrm{H}_{25} \mathrm{INO}_{2}[\mathrm{M}+\mathrm{H}]^{+}$requires $\mathrm{m} / z$ 486.0925, found $\mathrm{m} / z$, 486.0921 .

3.39. 2-[(2,3-Dimethylphenyl)amino]-5-[2-(thiophen-2-yl)propan-2-yl]benzoic acid, 3am.<smiles>Cc1cccc(Nc2ccccc2C(=O)O)c1C</smiles>

Mefenamic acid (1.0 equiv.)

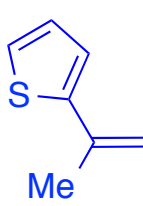

$2 \mathrm{~h}$ (2.0 equiv.)

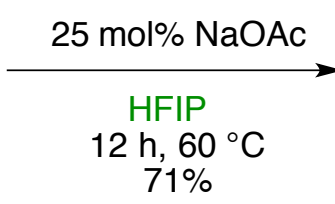

$71 \%$

From Mefenamic acid (48 mg, $0.2 \mathrm{mmol})$, alkene $\mathbf{2 h}(52 \mathrm{mg}, 0.4 \mathrm{mmol})$ and $\mathrm{NaOAc}(4.0 \mathrm{mg}$, $0.05 \mathrm{mmol}$ ), in $1.0 \mathrm{~mL}$ of HFIP, following the general procedure, aniline 3am was obtained. Chromatographic purification (gradient elution: 1:99 $\rightarrow$ 10:90 $\mathrm{MeOH}-\mathrm{CH}_{2} \mathrm{Cl}_{2}$ ) gave 3am (52 mg, $71 \%)$, as a yellow foam.

Data for 3am: $\boldsymbol{R}_{f} 0.40\left(5 \% \mathrm{MeOH}-\mathrm{CH}_{2} \mathrm{Cl}_{2}\right) .{ }^{1} \mathbf{H} \mathbf{~ N M R}\left(\mathbf{3 0 0} \mathbf{M H z}, \mathbf{C D C l}_{3}\right) \delta 10.93(1 \mathrm{H}$, br s, $\left.\mathrm{CO}_{2} \mathrm{H}\right), 9.06(1 \mathrm{H}$, br s, $\mathrm{NH}), 8.03(1 \mathrm{H}, \mathrm{d}, J=2.5 \mathrm{~Hz}, 6-\mathrm{H}), 7.21(1 \mathrm{H}, \mathrm{dd}, J=9.1$ and $2.6 \mathrm{~Hz}, 4-$ H), 7.11-7.16 (2 H, m, CH Thioph and 10-H), $7.08(1 \mathrm{H}, \mathrm{t}, J=7.5 \mathrm{~Hz}, 11-\mathrm{H}), 7.02(1 \mathrm{H}, \mathrm{d}, J=7.3$ $\mathrm{Hz}, 12-\mathrm{H}), 6.90(1 \mathrm{H}, \mathrm{dd}, J=5.1$ and $3.4 \mathrm{~Hz}, \mathrm{CH}$ Thioph $), 6.81(1 \mathrm{H}, \mathrm{dd}, J=3.5$ and $1.1 \mathrm{~Hz}, \mathrm{CH}$ Thioph), $6.68(1 \mathrm{H}, \mathrm{d}, J=9.0 \mathrm{~Hz}, 3-\mathrm{H}), 2.32(3 \mathrm{H}, \mathrm{s}, \mathrm{Me}), 2.18(3 \mathrm{H}, \mathrm{s}, \mathrm{Me}), 1.75(6 \mathrm{H}, \mathrm{s}, 2 \mathrm{x} \mathrm{Me})$. ${ }^{13} \mathrm{C}$ NMR (75 MHz, CDCl $) \delta 173.8\left(1 \mathrm{C}, \mathrm{CO}_{2} \mathrm{H}\right), 156.6(1 \mathrm{C}, \mathrm{C} \mathrm{Ar}), 148.8(1 \mathrm{C}, \mathrm{C} \mathrm{Ar}), 138.7(1 \mathrm{C}$, C Ar), 138.4 (1 C, C Ar), 137.6 (1 C, C Ar), 134.3 (1 C, C-4), 132.7 (1 C, C Ar), 128.9 (1 C, C-6), 127.1 (1 C, C-12), 126.4 (1 C, CH Thioph), 126.1 (1 C, C-11), 123.54 and 123.50 (2 C, CH Thioph and C-10), 123.3 (1 C, CH Thioph), 114.1 (1 C, C-3), 108.8 (1 C, C Ar), 40.9 (1 C, C-13), 32.0 (2 C, 2 x Me), 20.7 (1 C, Me), 14.2 (1 C, Me). HRMS (ESI): calculated for $\mathrm{C}_{22} \mathrm{H}_{24} \mathrm{NO}_{2} \mathrm{~S}[\mathrm{M}+\mathrm{H}]^{+}$requires $\mathrm{m} / \mathrm{z}$ 366.1522, found $\mathrm{m} / \mathrm{z} 366.1519$.

\section{Mechanistic studies.}

\subsection{Competition experiment}
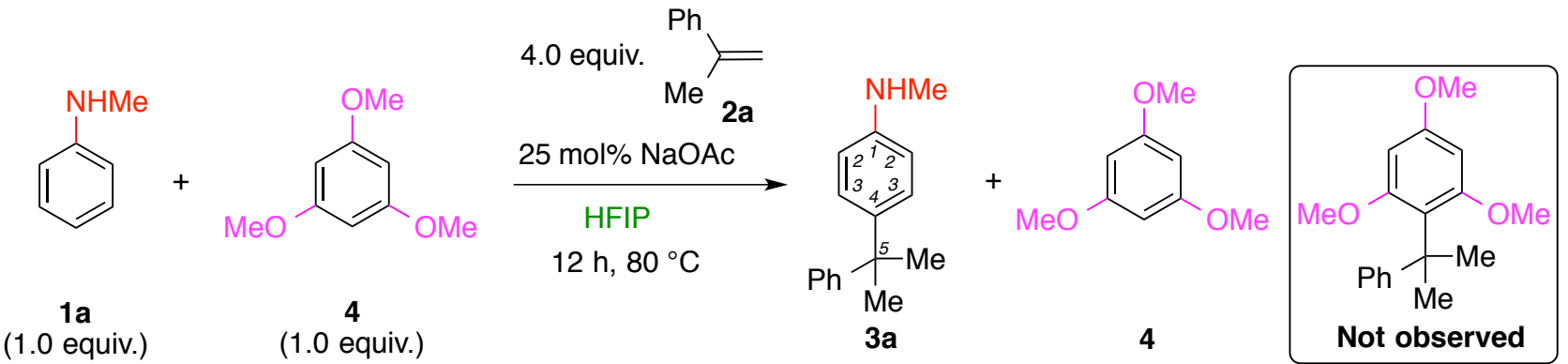
From aniline 1a (22 mg, $0.2 \mathrm{mmol}), 1,3,5$-trimethoxybenzene 4 (33 mg, $0.2 \mathrm{mmol})$, alkene 2a (104 $\mu \mathrm{l}, 0.8 \mathrm{mmol})$ and $\mathrm{NaOAc}(4.0 \mathrm{mg}, 0.05 \mathrm{mmol})$, in $1.0 \mathrm{~mL}$ of HFIP, following the general procedure, after solvent evaporation, the crude reaction was analized using ${ }^{1} \mathrm{H}$ NMR. Full conversion to aniline 3a (Figure S1, red boxes) and complete 1,3,5-trimethoxybenzene 4 recovered (Figure S1, purple boxes), was observed.

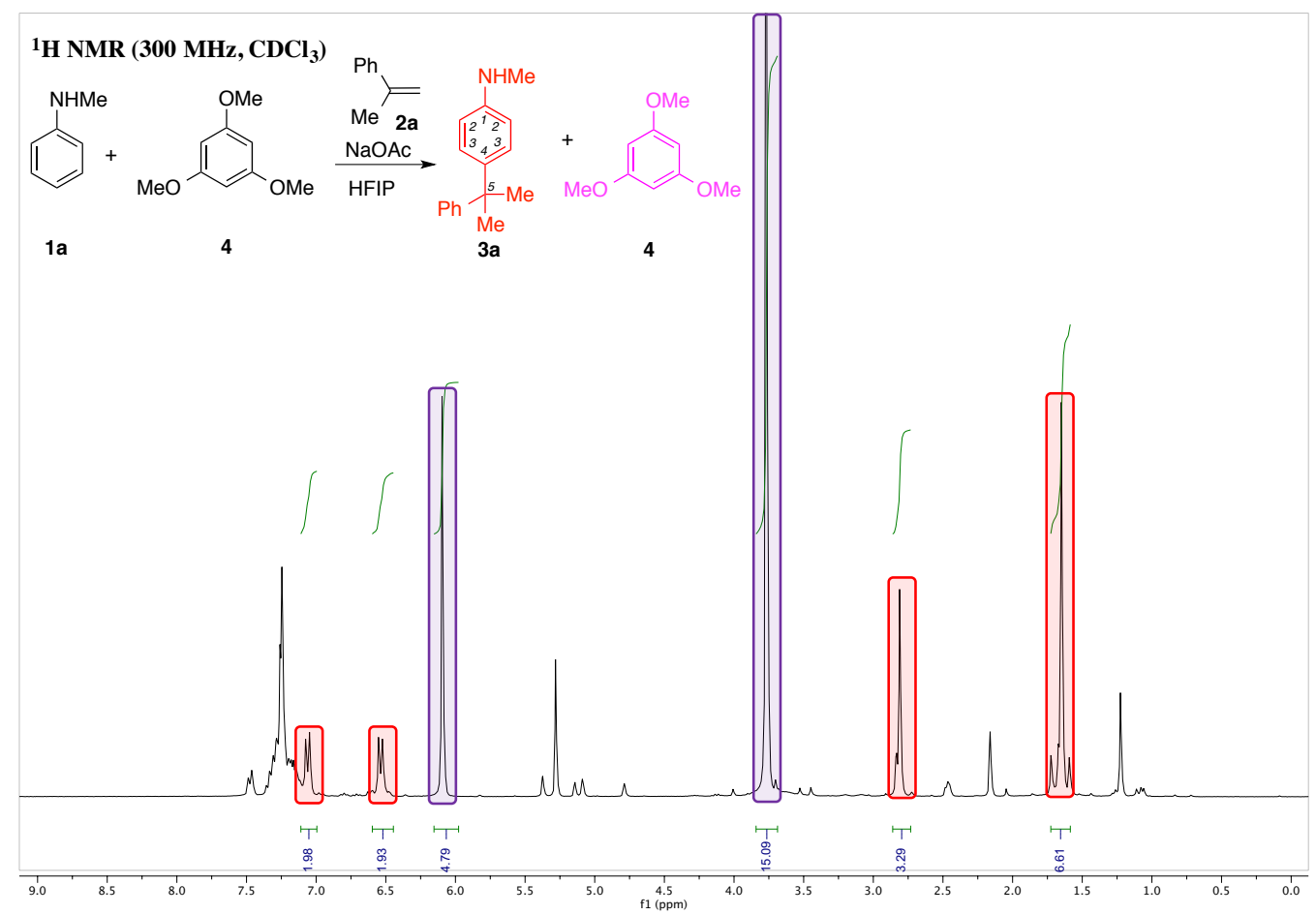

Figure S1: ${ }^{1} \mathrm{H}$ NMR of the crude competition reaction, after solvent evaporation.

\subsection{Deuterium labelling experiments}

4.2.1. $\quad N$-Methyl-4-(2-phenylpropan-2-yl-1- $d$ )benzen-2,6- $d_{2}$-amine, 3a-D ${ }_{3}$ and $N$-Methyl-4(2-phenylpropan-2-yl-1-d)aniline, 3a-D.<smiles>CNc1ccccc1</smiles>

$1 \mathrm{a}$

(1.0 equiv.)<smiles>C=C(C)Pc1ccccc1</smiles>

2a

(2.0 equiv.)

$25 \mathrm{~mol} \% \mathrm{NaOAc}$

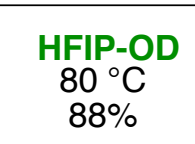

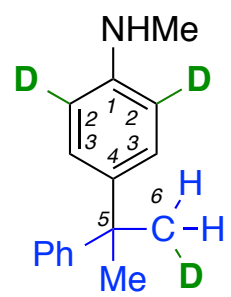

$3 a-D_{3}$

$(80)$

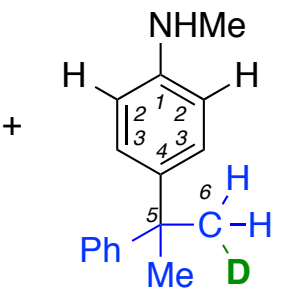

3a-D

(20)

From aniline 1a (22 mg, $0.2 \mathrm{mmol})$, alkene $2 \mathbf{a}(52 \mu \mathrm{l}, 0.4 \mathrm{mmol})$ and $\mathrm{NaOAc}(4.0 \mathrm{mg}, 0.05$ $\mathrm{mmol}$ ), in $1.0 \mathrm{~mL}$ of HFIP-OD, following the general procedure, aniline 3a with a quantitative deuterium incorporation in the C-6 position and an $80 \%$ deuterium incorporation in the C-2 positions was obtained. Chromatographic purification (gradient elution: 5:95 $\rightarrow$ 30:70 $\mathrm{Et}_{2} \mathrm{O}$ - hexane) gave an $80: 20$ ratio of $\mathbf{3 a}-\mathbf{D}_{\mathbf{3}}: \mathbf{3 a}-\mathbf{D}$ (40 mg, $88 \%$ ), as a yellow oil. 
Data for $\mathbf{3 a}-\mathbf{D}_{\mathbf{3}}$ (from the mixture): $\boldsymbol{R}_{f} 0.40\left(25 \% \mathrm{Et}_{2} \mathrm{O}\right.$ - hexane). ${ }^{1} \mathbf{H}$ NMR (400 $\mathbf{M H z}, \mathbf{C D C l}_{\mathbf{3}}$ )

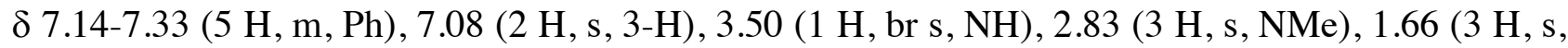
Me), 1.64 (2 H, s, 6-H $).{ }^{13} \mathbf{C}$ NMR (100 MHz, CDCl $_{3}$ ) $\delta 151.5$ (1 C, C Ar), 146.97 (1 C, C Ar), 139.8 (1 C, C Ar), 128.0 (2 C, CH Ph), 127.6 (2 C, C-3), 126.9 (2 C, CH Ph), 125.5 (1 C, CH Ph), 112.0 (2 $\left.\mathrm{C}, \mathrm{t}, J_{C-D}=23.8 \mathrm{~Hz}, \mathrm{C}-2\right), 42.15(1 \mathrm{C}, \mathrm{C}-5), 31.1(1 \mathrm{C}, \mathrm{NMe}), 31.01(1 \mathrm{C}, \mathrm{Me}), 30.70\left(1 \mathrm{C}, \mathrm{t}, J_{C-D}=\right.$ 19.5 Hz, C-6). ). ${ }^{2} \mathbf{H}$ NMR (76.8 $\left.\mathbf{M H z}, \mathbf{C H C l}_{3}\right) \delta 6.65$ (2 D, s, 2-D), 1.66 (1 D, s, 6-D).

Partial data for 3a-D (from the mixture): The NMR signals overlapped with those of $\mathbf{3 a}-\mathbf{D}_{\mathbf{3}}$, except for: ${ }^{1} \mathbf{H}$ NMR (400 MHz, $\left.\mathbf{C D C l}_{3}\right) \delta 6.56(2 \mathrm{H}, \mathrm{d}, J=8.9 \mathrm{~Hz}, 2-\mathrm{H}) .{ }^{13} \mathbf{C}$ NMR (100 MHz, $\left.\mathbf{C D C l}_{3}\right) \delta 147.02$ (1 C, C Ar), 127.7 (2 C, C-3), 112.3 (2 C, C-2), 42.23 (1 C, C-5), 30.97 (1 C, Me).

4.2.2. $N$-Methylbenzen-2,4,6- $d_{3}$-amine, $1 \mathrm{a}-\mathrm{D}_{3}$.

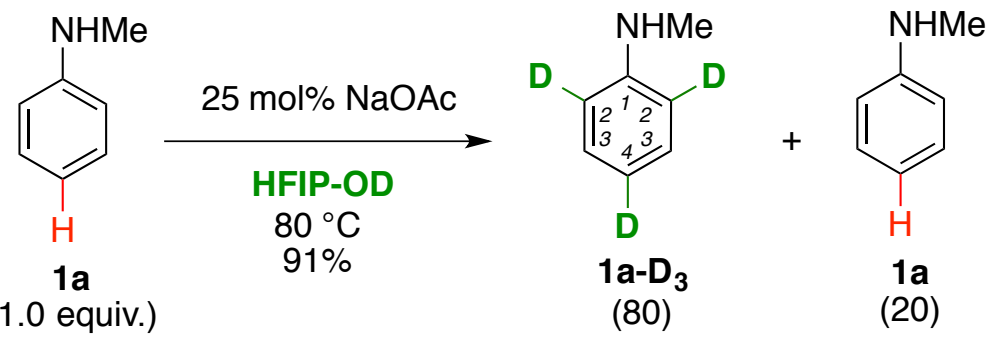

From aniline $1 \mathrm{a}(22 \mathrm{mg}, 0.2 \mathrm{mmol})$ and NaOAc $(4.0 \mathrm{mg}, 0.05 \mathrm{mmol})$, in $1.0 \mathrm{~mL}$ of HFIP-OD, following the general procedure, aniline 1a with an $80 \%$ deuterium incorporation in the C-2 and C-6 positions was obtained. Chromatographic purification (gradient elution: 5:95 $\rightarrow$ 30:70 $\mathrm{Et}_{2} \mathrm{O}$ - hexane)

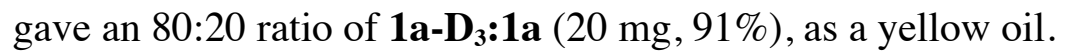

Data for $\mathbf{1 a}-\mathbf{D}_{\mathbf{3}}$ (from the mixture): $\boldsymbol{R}_{f} 0.40\left(25 \% \mathrm{Et}_{2} \mathrm{O}\right.$ - hexane). ${ }^{1} \mathbf{H}$ NMR (400 $\mathbf{M H z}, \mathbf{C D C l}_{3}$ ) $\delta 7.21(2 \mathrm{H}, \mathrm{s}, 3-\mathrm{H}), 3.68\left(1 \mathrm{H}, \mathrm{br}\right.$ s, NH), $2.85(3 \mathrm{H}, \mathrm{s}, \mathrm{NMe}) .{ }^{13} \mathbf{C}$ NMR (100 MHz, CDCl $) \delta 149.3$ $\left(1 \mathrm{C}, \mathrm{t}, J_{C-D}=5.3 \mathrm{~Hz}, \mathrm{C}-1\right), 129.2\left(2 \mathrm{C}, \mathrm{t}, J_{C-D}=10.7 \mathrm{~Hz}, \mathrm{C}-3\right), 117.3\left(1 \mathrm{C}, \mathrm{t}, J_{C-D}=26.1 \mathrm{~Hz}, \mathrm{C}-4\right)$, $112.3\left(2 \mathrm{C}, \mathrm{t}, J_{C-D}=24.2 \mathrm{~Hz}, \mathrm{C}-2\right), 30.9(1 \mathrm{C}, \mathrm{NMe}) .{ }^{2} \mathbf{H}$ NMR (76.8 MHz, $\left.\mathbf{C H C l}_{3}\right) \delta 6.78(1 \mathrm{D}, \mathrm{s}, 4-$ D), 6.68 (2 D, s, 2-D).

4.2.3. 4-[1-(4-Methoxyphenyl)propyl-2- $d]-N$-methylbenzen-2,6- $d_{2}$-amine, 3ai-D ${ }_{3}$ and 4-[1(4-Methoxyphenyl)propyl-2- $d]-N$-methylaniline, 3ai-D.

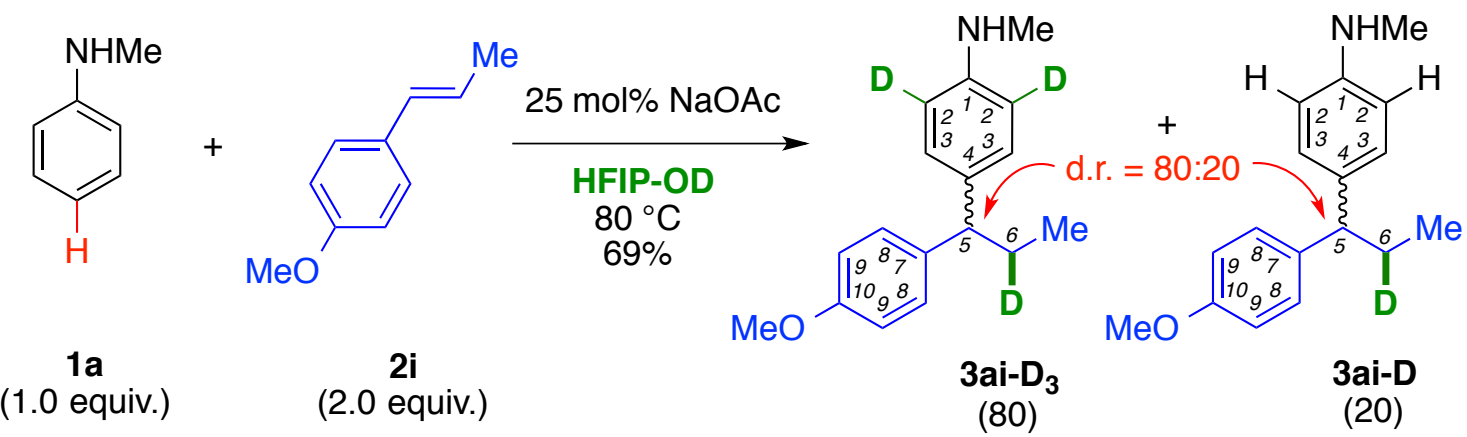


From aniline 1a (22 mg, $0.2 \mathrm{mmol})$, alkene $2 \mathbf{i}(60 \mathrm{mg}, 0.4 \mathrm{mmol})$ and $\mathrm{NaOAc}(4.0 \mathrm{mg}, 0.05$ $\mathrm{mmol}$ ), in $1.0 \mathrm{~mL}$ of HFIP-OD, following the general procedure, aniline 3ai with a quantitative deuterium incorporation in the C-6 position and an $80 \%$ deuterium incorporation in the C-2 position was obtained. Chromatographic purification (gradient elution: 5:95 $\rightarrow$ 30:70 $\mathrm{Et}_{2} \mathrm{O}$ - hexane) gave an 80:20 ratio of 3ai- $\mathbf{D}_{3}: 3 \mathbf{3 i}-\mathbf{D}$, each one as an 80:20 mixture of diastereoisomers (35 $\mathrm{mg}, 69 \%$ ), as a yellow oil.

Data for 3ai-D $\mathbf{D}_{\mathbf{3}}$ (from the mixture): $\boldsymbol{R}_{f} 0.40\left(25 \% \mathrm{Et}_{2} \mathrm{O}-\right.$ hexane). ${ }^{1} \mathbf{H}$ NMR (400 $\mathbf{~ M H z}$, $\left.\mathbf{C D C l}_{3}\right) \delta 7.13(2 \mathrm{H}, \mathrm{d}, J=8.6 \mathrm{~Hz}, 8-\mathrm{H}), 7.05(2 \mathrm{H}, \mathrm{s}, 3-\mathrm{H}), 6.81(2 \mathrm{H}, \mathrm{d}, J=8.5 \mathrm{~Hz}, 9-\mathrm{H}), 3.77(3 \mathrm{H}$, s, OMe), $3.66(1 \mathrm{H}, \mathrm{d}, J=7.1 \mathrm{~Hz}, 5-\mathrm{H}$ minor diast. $), 3.64(1 \mathrm{H}, \mathrm{d}, J=7.5 \mathrm{~Hz}, 5-\mathrm{H}$ major diast.), 2.81 (3 H, s, NMe), 1.99 (1 H, quint, $J=7.4 \mathrm{~Hz}, 6-\mathrm{H}), 0.89$ (3 H, d, $J=7.1 \mathrm{~Hz}$, Me minor diast.), 0.87 (3 $\mathrm{H}, \mathrm{d}, J=7.2 \mathrm{~Hz}$, Me major diast.). ${ }^{13} \mathbf{C}$ NMR (100 MHz, CDCl $) \delta 157.7$ (1 C, C Ar), 146.9 (1 C, C Ar), 138.3 (1 C, C Ar), 135.1 (1 C, C Ar), 128.8 (2 C, C-8), 128.6 (2 C, C-3), 113.8 (2 C, C-9), 112.8 $\left(2 \mathrm{C}, \mathrm{t}, J_{C-D}=24.0 \mathrm{~Hz}, \mathrm{C}-2\right), 55.3(1 \mathrm{C}, \mathrm{OMe}), 51.5(1 \mathrm{C}, \mathrm{C}-5), 31.4(1 \mathrm{C}, \mathrm{NMe}), 28.7\left(1 \mathrm{C}, \mathrm{t}, J_{C-D}=\right.$ 19.6 Hz, C-6), 12.9 (1 C, Me). ${ }^{2} \mathbf{H}$ NMR (76.8 MHz, $\left.\mathbf{C H C l}_{3}\right) \delta 6.65$ (2 D, s, 2-D), 1.98 (1 D, s, 6-D).

Partial data for 3ai-D (from the mixture): The NMR signals overlapped with those of $\mathbf{3 a i}-\mathbf{D}_{\mathbf{3}}$, except for: ${ }^{1} \mathbf{H}$ NMR (400 MHz, $\left.\mathbf{C D C l}_{3}\right) \delta 6.59(2 \mathrm{H}, \mathrm{d}, J=8.8 \mathrm{~Hz}, 2-\mathrm{H}) .{ }^{13} \mathbf{C}$ NMR (100 MHz, $\mathbf{C D C l}_{3}$ ) $\delta 147.0$ (1 C, C Ar), 128.7 (2 C, C-3), 113.1 (2 C, C-2), 51.6 (1 C, C-5), 29.1 (1 C, C-6), 13.0 $(1 \mathrm{C}, \mathrm{Me})$.

\subsubsection{2,6-Diisopropyl-4-[1-(4-methoxyphenyl)propyl-2- $d]$ aniline, 3an-D.}

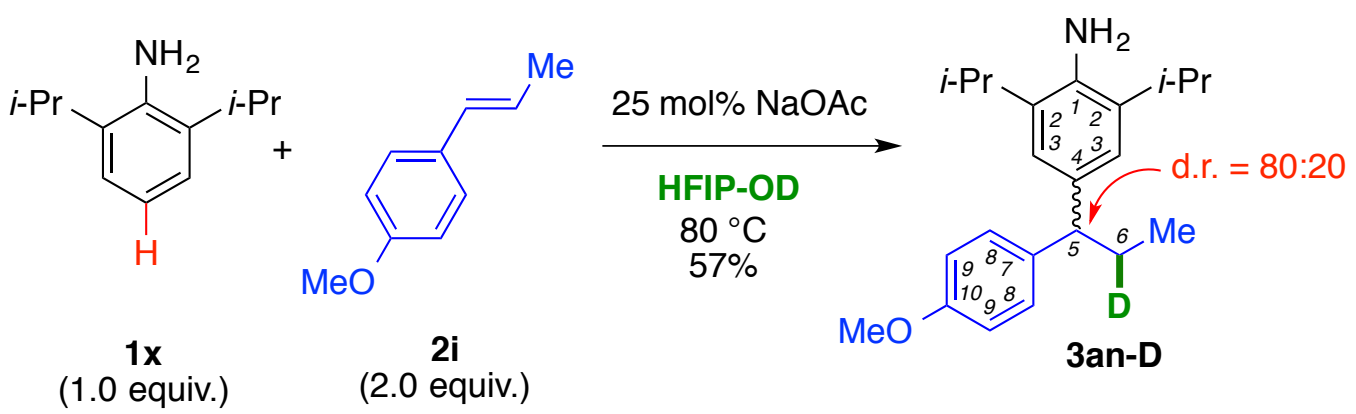

From aniline 1x (36 mg, $0.2 \mathrm{mmol})$, alkene $2 \mathbf{i}(60 \mathrm{mg}, 0.4 \mathrm{mmol})$ and $\mathrm{NaOAc}(4.0 \mathrm{mg}, 0.05$ $\mathrm{mmol}$ ), in $1.0 \mathrm{~mL}$ of HFIP-OD, following the general procedure, aniline 3an-D with a quantitative deuterium incorporation in the C-6 position was obtained. Chromatographic purification (gradient elution: 5:95 $\rightarrow$ 30:70 $\mathrm{Et}_{2} \mathrm{O}$ - hexane) gave an 80:20 ratio of diastereoisomers 3an-D (37 mg, 57\%), as a yellow oil.

Data for 3an-D: $\boldsymbol{R}_{f} 0.40\left(25 \% \mathrm{Et}_{2} \mathrm{O}\right.$ - hexane). ${ }^{1} \mathbf{H}$ NMR (400 $\left.\mathbf{M H z}, \mathbf{C D C l}_{3}\right) \delta 7.14(2 \mathrm{H}, \mathrm{d}, J$ $=8.6 \mathrm{~Hz}, 8-\mathrm{H}), 6.88(2 \mathrm{H}, \mathrm{s}, 3-\mathrm{H}), 6.81(2 \mathrm{H}, \mathrm{d}, J=8.7 \mathrm{~Hz}, 9-\mathrm{H}), 3.77(3 \mathrm{H}, \mathrm{s}, \mathrm{OMe}), 3.66(1 \mathrm{H}, \mathrm{d}, J$ $=6.9 \mathrm{~Hz}, 5-\mathrm{H}$ minor diast. $), 3.64(1 \mathrm{H}, \mathrm{d}, J=7.6 \mathrm{~Hz}, 5-\mathrm{H}$ major diast. $), 2.92(2 \mathrm{H}$, sept, $J=6.7 \mathrm{~Hz}$, 
2 x CH $i$-Pr), 1.99 (1 H, quint, $J=7.4 \mathrm{~Hz}, 6-\mathrm{H}$ minor diast. $), 1.98(1 \mathrm{H}$, quint, $J=7.4 \mathrm{~Hz}, 6-\mathrm{H}$ major diast.), $1.24\left(12 \mathrm{H}, \mathrm{d}, J=8.7 \mathrm{~Hz}, 4 \times \mathrm{CH}_{3} i\right.$-Pr $), 0.88(3 \mathrm{H}, \mathrm{d}, J=7.0 \mathrm{~Hz}, \mathrm{Me}$ minor diast.), $0.86(3 \mathrm{H}$, d, $J=7.2 \mathrm{~Hz}$, Me major diast.). ${ }^{13} \mathbf{C}$ NMR (100 MHz, $\left.\mathbf{C D C l}_{3}\right) \delta 157.7$ (1 C, C Ar), 138.4 (1 C, C Ar), 135.8 (1 C, C Ar), 132.8 (1 C, C Ar), 132.7 (2 C, C-2), 128.9 (2 C, C-8), 122.3 (2 C, C-3), 113.7 (2 C, C-9), 55.4 (1 C, OMe), 55.4 (1 C, C-5 minor diast.), 52.3 (1 C, C-5 major diast.), 29.1 (1 C, t, $J_{C-}$ $\left.{ }_{D}=19.5 \mathrm{~Hz}, \mathrm{C}-6\right), 28.2$ (2 C, 2 x CH $i$-Pr), 22.7 (4 C, 4 x CH $3 i$-Pr), 13.07 (1 C, Me minor diast.), 12.97 (1 C, Me major diast.). ${ }^{2} \mathbf{H}$ NMR (76.8 $\left.\mathbf{M H z}, \mathbf{C H C l}_{3}\right) \delta 1.98$ (1 D, s, 6-D). HRMS (ESI): calculated for $\mathrm{C}_{22} \mathrm{H}_{31} \mathrm{DNO}[\mathrm{M}+\mathrm{H}]^{+}$requires $m / z$ 327.2530, found $m / z$ 327.2531.

\subsection{NMR experiments}

\subsection{1. ${ }^{1}$ H-NMR of aniline 1a, HFIP and binary mixture.}

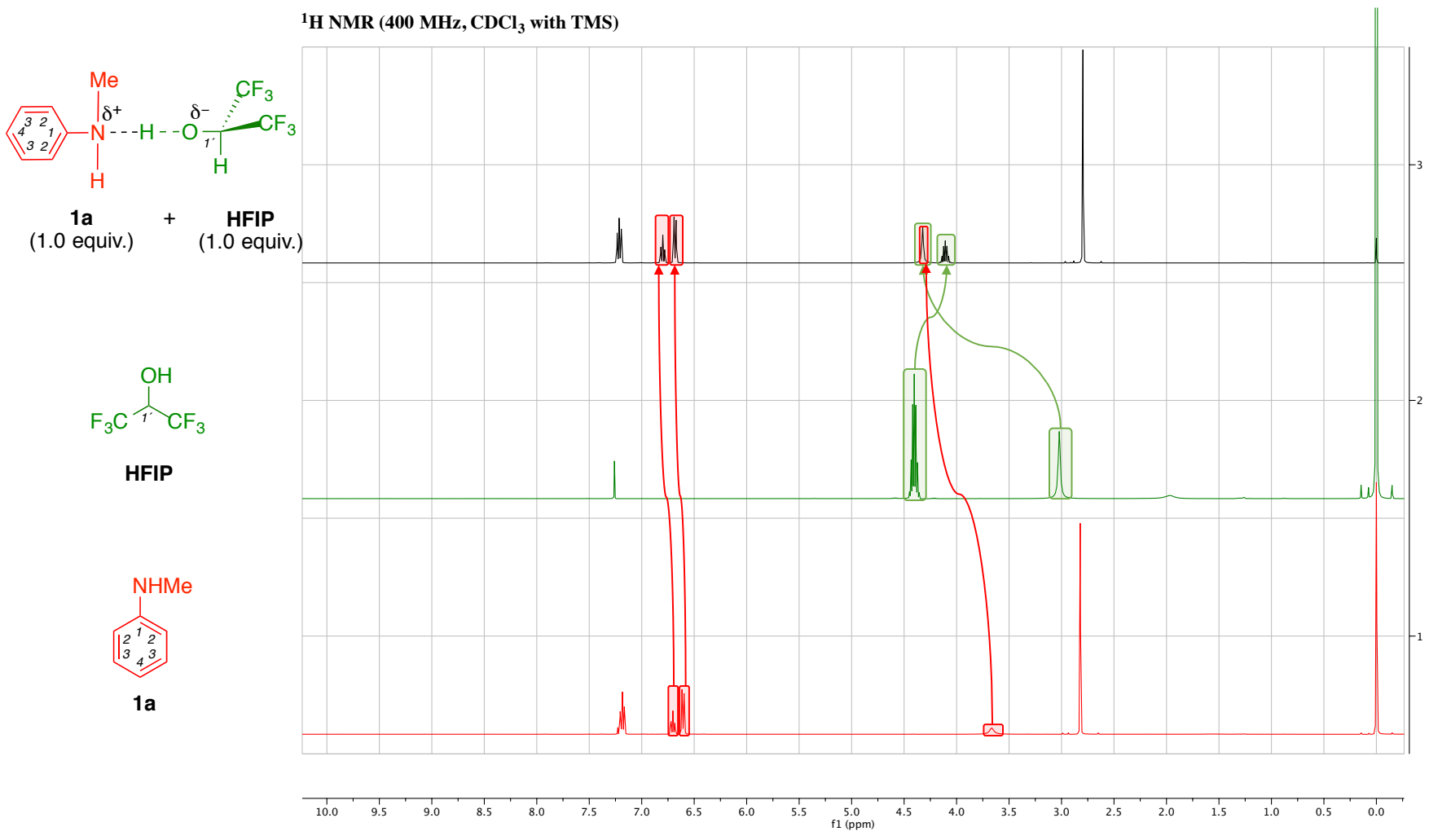

\begin{tabular}{c|c|c|c|c} 
& Aniline & HFIP & Mixture & $\Delta \boldsymbol{\delta}$ \\
\hline $\mathbf{2 - H}$ & $6.61 \mathrm{~d}(J=7.6)$ & - & $6.68 \mathrm{~d}(J=7.6)$ & $\mathbf{0 . 0 7}$ \\
\hline $\mathbf{3 - H}$ & $7.20 \mathrm{t}(J=7.5)$ & - & $7.21 \mathrm{t}(J=7.5)$ & 0.01 \\
\hline $\mathbf{4 - H}$ & $6.70 \mathrm{t}(J=7.4)$ & - & $6.80 \mathrm{t}(J=7.4)$ & $\mathbf{0 . 1 0}$ \\
\hline $\mathbf{N H}$ & $3.67 \mathrm{br} \mathrm{s}$ & - & $4.32 \mathrm{br} \mathrm{s}$ & $\mathbf{0 . 6 5}$ \\
\hline $\mathbf{M e}$ & $2.82 \mathrm{~s}$ & - & $2.80 \mathrm{~s}$ & -0.02 \\
\hline $\mathbf{O H}$ & - & $3.02 \mathrm{br} \mathrm{s}$ & $4.32 \mathrm{br} \mathrm{s}$ & $\mathbf{1 . 3}$ \\
\hline $\mathbf{1}^{\prime}-\mathbf{H}$ & - & 4.40 hept $(J=6.0)$ & 4.11 hept $(J=6.0)$ & $\mathbf{- 0 . 2 9}$
\end{tabular}


The most significant changes found in ${ }^{1} \mathrm{H}$ NMR, for the binary mixture compared to the individual species, are the downfield shift (deshieled) of the O-H proton, from a frequency of 3.02 ppm to $4.32 \mathrm{ppm}(\Delta \delta=0.65)$ and the $\mathrm{N}-\mathrm{H}$ proton, from a frequency of $3.02 \mathrm{ppm}$ to $4.32 \mathrm{ppm}(\Delta \delta=$ 1.3). Slightly less significant changes are found in $2-\mathrm{H}, 3-\mathrm{H}$ belonging to the aniline and $1^{\prime}-\mathrm{H}$ to the HFIP moieties, with a smaller shifting of $0.07,0.10$ and -0.29 ppm respectively.

These data support a H-bonding between the basic amino group of the aniline and the exceptionally good hydrogen bond donor HFIP. Both the NH and the $\mathrm{OH}$ are deshielded and appear at the same frequency of $4.32 \mathrm{ppm}$.

\subsection{2. ${ }^{1} \mathrm{H}-\mathrm{NMR}$ of $\mathrm{Bu}_{4} \mathrm{NOAc}$, HFIP and binary mixture.}

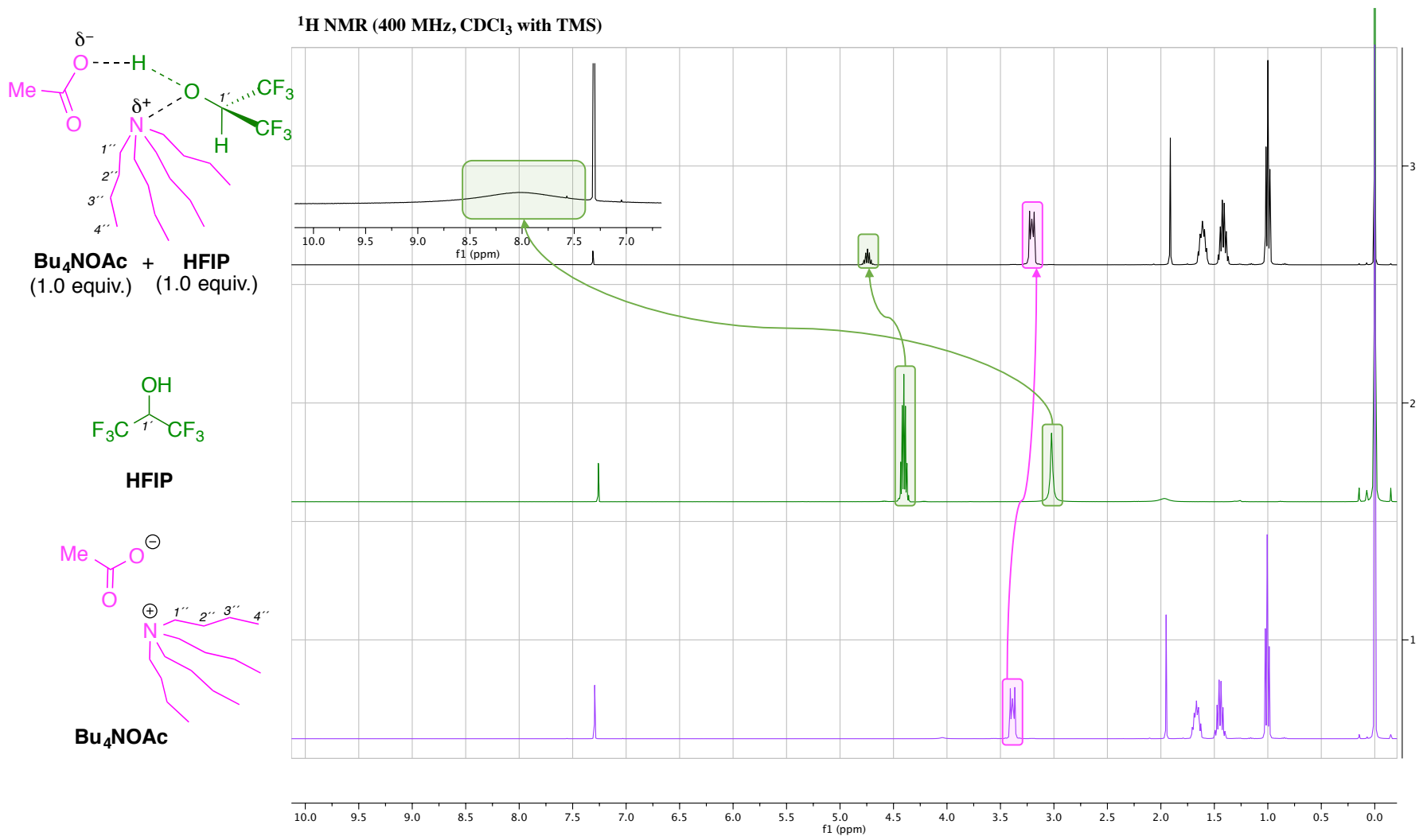

\begin{tabular}{|c|c|c|c|c|}
\hline & $\mathrm{Bu}_{4} \mathrm{NOAc}$ & HFIP & Mixture & $\Delta \delta$ \\
\hline 1"-H & $3.39 \mathrm{t}(J=8.5)$ & - & $3.21 \mathrm{t}(J=8.4)$ & -0.18 \\
\hline $2 "-H$ & 1.67 quint $(J=8.1)$ & - & 1.61 quint $(J=7.8)$ & -0.06 \\
\hline $3^{\prime \prime}-\mathrm{H}$ & $1.45 \operatorname{sext}(J=7.4)$ & - & $1.42 \operatorname{sext}(J=7.3)$ & -0.03 \\
\hline $44^{\prime \prime}-\mathrm{H}$ & $1.01 \mathrm{t}(J=7.4)$ & - & $1.00 \mathrm{t}(J=7.3)$ & -0.01 \\
\hline Me-OAc & $1.95 \mathrm{~s}$ & - & $1.91 \mathrm{~s}$ & -0.04 \\
\hline $\mathbf{O H}$ & - & $3.02 \mathrm{br} \mathrm{s}$ & 8.01 br s & 4.99 \\
\hline $\mathbf{1}^{\prime}-\mathrm{H}$ & - & 4.40 hept $(J=6.0)$ & 4.74 hept $(J=6.8)$ & 0.34 \\
\hline
\end{tabular}


The most significant changes found in ${ }^{1} \mathrm{H}$ NMR, for the binary mixture compared to the individual species, are the downfield shift (deshieled) of the $\mathrm{OH}$, from a frequency of $3.02 \mathrm{ppm}$ to $8.01 \mathrm{ppm}(\Delta \delta=4.99)$ and the $\mathrm{CH}$ of HFIP, from a frequency of $4.40 \mathrm{ppm}$ to $4.74 \mathrm{ppm}(\Delta \delta=0.34)$. Additionally upfield shift (shieled) of the butyl and acetyl moieties of the $\mathrm{Bu}_{4} \mathrm{NOAc}(\Delta \delta=-0.04$ to $0.18)$ is observed.

These data support a H-bonding between the basic acetate group and the exceptionally good hydrogen bond donor HFIP. The proton transfer is not complete, as acetic acid (AcOH) is not observed (carboxylic acid $\mathrm{OH}$ in $\mathrm{CDCl}_{3}$ appears at $12 \mathrm{ppm}$ ). Both the $\mathrm{OH}$ and the $\mathrm{CH}$ of $\mathrm{HFIP}$ are deshielded, what might indicate not only the $\mathrm{H}$-bonding between the acetate and $\mathrm{OH}$, but also some degree of interaction between the cationic $\mathrm{Bu}_{4} \mathrm{~N}^{+}$and the Hexafluoroisopropoxide moieties.

\subsection{3. ${ }^{1} \mathrm{H}-\mathrm{NMR}$ of alkene $2 \mathrm{a}, \mathrm{HFIP}$ and binary mixture.}

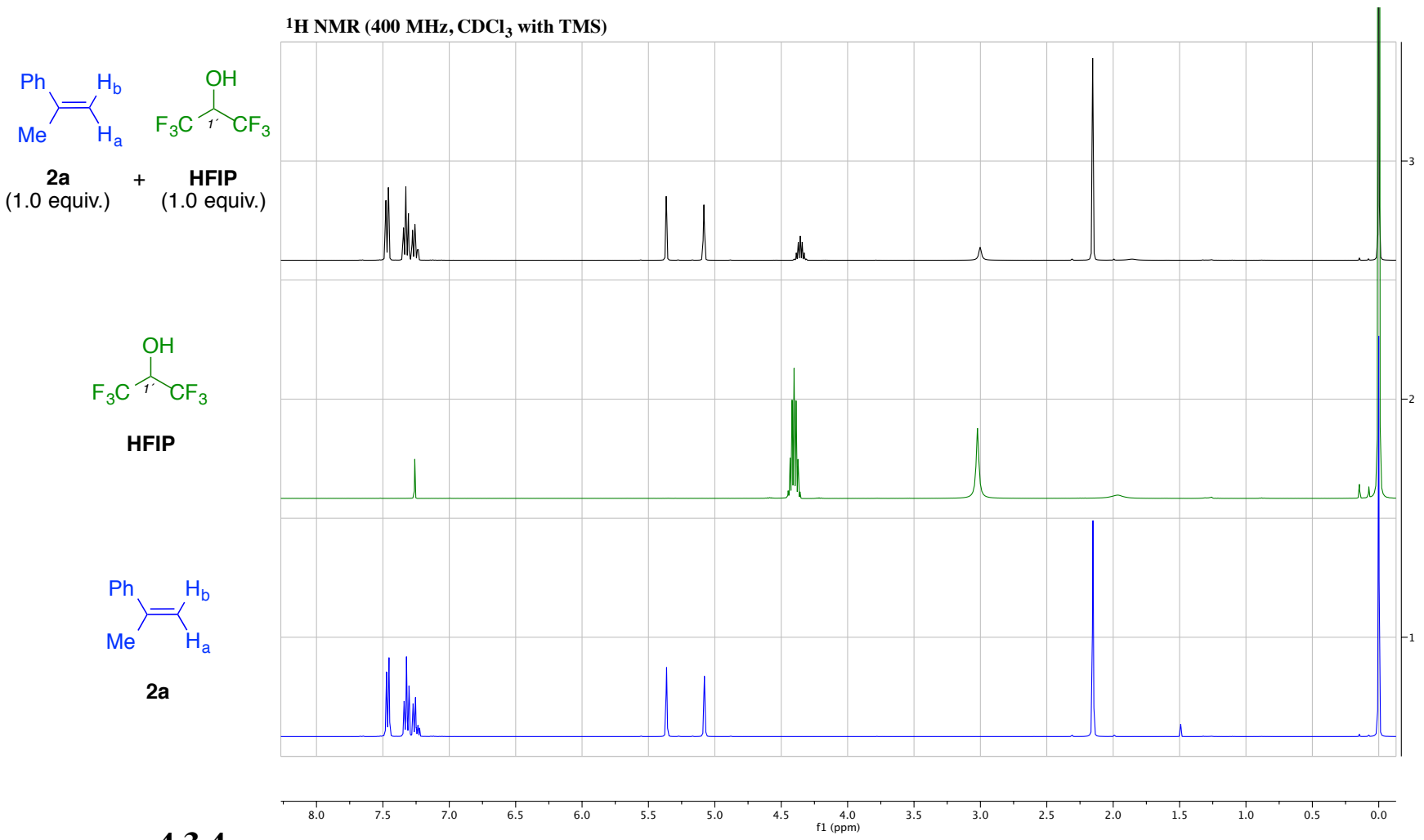

4.3.4.

\begin{tabular}{c|c|c|c|c} 
& Alkene & HFIP & Mixture & $\Delta \boldsymbol{\delta}$ \\
\hline Ha & $5.37 \mathrm{~s}$ & - & $5.37 \mathrm{~s}$ & 0 \\
\hline $\mathbf{H b}$ & $5.08 \mathrm{~s}$ & - & $5.08 \mathrm{~s}$ & 0 \\
\hline Me & $2.15 \mathrm{~s}$ & - & $2.15 \mathrm{~s}$ & 0 \\
\hline $\mathbf{O H}$ & - & 3.02 br s & $3.00 \mathrm{br} \mathrm{s}$ & -0.02 \\
\hline $\mathbf{1}^{\prime}-\mathbf{H}$ & - & 4.40 hept $(J=6.0)$ & 4.36 hept $(J=6.0)$ & -0.04
\end{tabular}

No significant changes are found in ${ }^{1} \mathrm{H}$ NMR. 
4.3.5. ${ }^{1} \mathrm{H}-\mathrm{NMR}$ of HFIP, mixtures of: a) $1 \mathrm{a}+2 \mathrm{a}+\mathrm{Bu}_{4} \mathrm{NOAc}$, b) $1 \mathrm{a}+2 \mathrm{a}+\mathrm{Bu}_{4} \mathrm{NOAc}+$ HFIP.

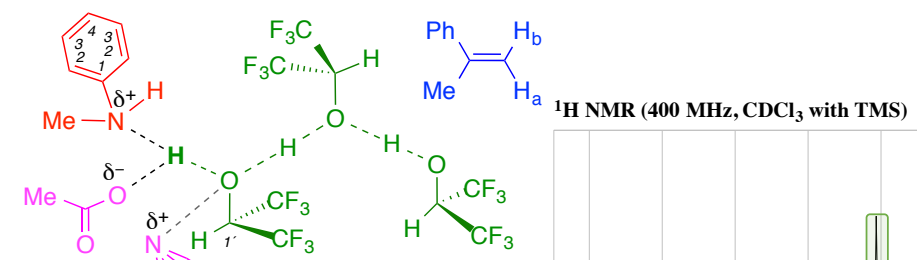

$1 \mathrm{a}+2 \mathrm{a}^{4 \prime}+\mathrm{Bu}_{4} \mathrm{NOAc}+\mathrm{HFIP}$

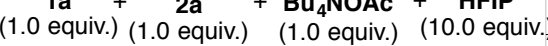
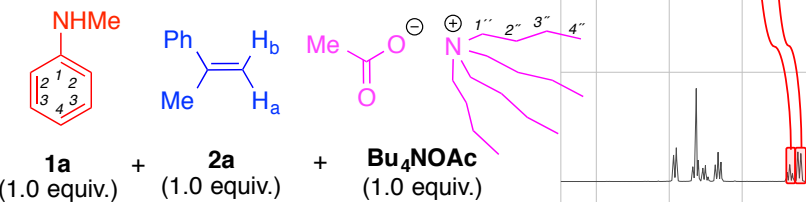

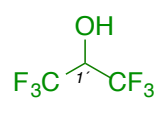

HFIP

\begin{tabular}{|c|c|c|c|c|}
\hline & HFIP & Mixture w/o HFIP & Mixture with HFIP & $\Delta \delta$ \\
\hline $2-H$ & - & $6.61 \mathrm{~d}(J=7.6)$ & $6.78 \mathrm{~d}(J=7.5)$ & 0.17 \\
\hline 3-H & - & $7.16 \mathrm{t}(J=7.5)$ & $7.33 \mathrm{t}(J=7.4)$ & 0.23 \\
\hline $4-H$ & - & $6.67 \mathrm{t}(J=7.3)$ & $6.90 \mathrm{t}(J=7.4)$ & 0.23 \\
\hline Me (aniline) & - & $2.80 \mathrm{~s}$ & $2.82 \mathrm{~s}$ & 0.02 \\
\hline 1"'-H & - & $3.31 \mathrm{t}(J=8.5)$ & $3.01 \mathrm{t}(J=8.5)$ & -0.30 \\
\hline $2 " \prime-H$ & - & 1.62 quint $(J=8.1)$ & 1.54 quint $(J=8.1)$ & -0.08 \\
\hline $3{ }^{\prime \prime}-\mathrm{H}$ & - & $1.42 \operatorname{sext}(J=7.3)$ & $1.38 \operatorname{sext}(J=7.3)$ & -0.04 \\
\hline $4 " \prime-H$ & - & $0.99 \mathrm{t}(J=7.3)$ & $0.99 \mathrm{t}(J=7.3)$ & 0 \\
\hline Me (OAc) & - & $1.95 \mathrm{~s}$ & $1.93 \mathrm{~s}$ & -0.02 \\
\hline Ha & - & $5.37 \mathrm{~s}$ & $5.37 \mathrm{~s}$ & 0 \\
\hline $\mathbf{H b}$ & - & $5.08 \mathrm{~s}$ & $5.08 \mathrm{~s}$ & 0 \\
\hline Me (alkene) & - & $2.15 \mathrm{~s}$ & $2.15 \mathrm{~s}$ & 0 \\
\hline OH-HFIP & 3.02 br s & - & 6.03 br s & 3.01 \\
\hline $1^{\prime}-\mathrm{H}$ & 4.40 hept $(J=6.0)$ & - & 4.31 hept $(J=6.2)$ & -0.09 \\
\hline
\end{tabular}

The most significant changes found in ${ }^{1} \mathrm{H}$ NMR, for the mixture containing HFIP compared to the one without HFIP, are the downfield shift (deshieled) of the $\mathrm{OH}$, from a frequency of $3.02 \mathrm{ppm}$ to $6.03 \mathrm{ppm}(\Delta \delta=3.01)$ and the $2-\mathrm{H}, 3-\mathrm{H}, 4-\mathrm{H}$ belonging to the aniline with shifting of $0.17,0.23$ and $0.23 \mathrm{ppm}$ respectively. Additionally upfield shift (shielded) of the butyl and acetyl moieties of 
the $\mathrm{Bu}_{4} \mathrm{NOAc}(\Delta \delta=-0.02$ to -0.30$)$ and $\mathrm{CH}$ of $\operatorname{HFIP}(\Delta \delta=-0.09)$ is observed.

These data follow a similar trend than the binary mixtures and support a H-bonding between the aniline and the acetate moieties with the hydrogen bond donor HFIP. While the aniline and the $\mathrm{OH}$ are deshielded (indicating a less electronic environment) the tretrabutylammonium is shielded (probably because of the hexafluoroisopropoxy interaction).

\subsubsection{NOESY-2D experiment.}

Additional support for the H-bonded structure is found upon inspection of the NOESY-2D spectra of the mixture: aniline 1a (1.0 equiv.) + alkene 2a (1.0 equiv.) + Bun NOAc (1.0 equiv.) + HFIP (10.0 equiv. to better simulate the reaction conditions where pure HFIP is used).

The NOESY-2D shows interactions between both the $\mathrm{CH}\left(1^{\prime}-\mathrm{H}\right)$ and $\mathrm{OH}$ of HFIP with the butyl moiety (1"'-H, 2"'-H, 3"'-H and 4"'-H) and the Me-OAc of the Bu${ }_{4} N O A c$ (purple boxes in Figure S2). These data confirm the intermolecular spatial connection between HFIP and $\mathrm{Bu}_{4} \mathrm{NOAc}$.

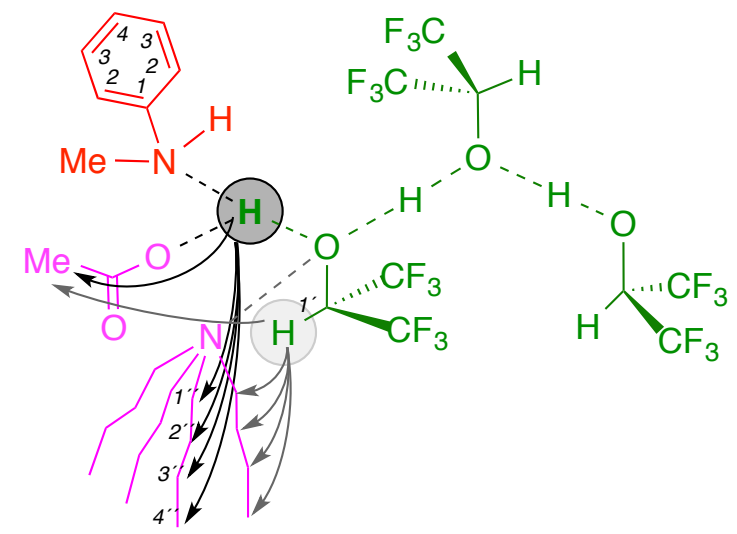

Additionally, cross-peaks between both $\mathrm{CH}$ $\left(1^{\prime}-\mathrm{H}\right)$ and $\mathrm{OH}$ of HFIP with the Me of the aniline. Moreover, there is an interesting cross-peak, albeit with less intensity, between the $\mathrm{CH}\left(1^{\prime}-\mathrm{H}\right)$ and the aromatic 2-H of the aniline (red boxes in Figure S2)

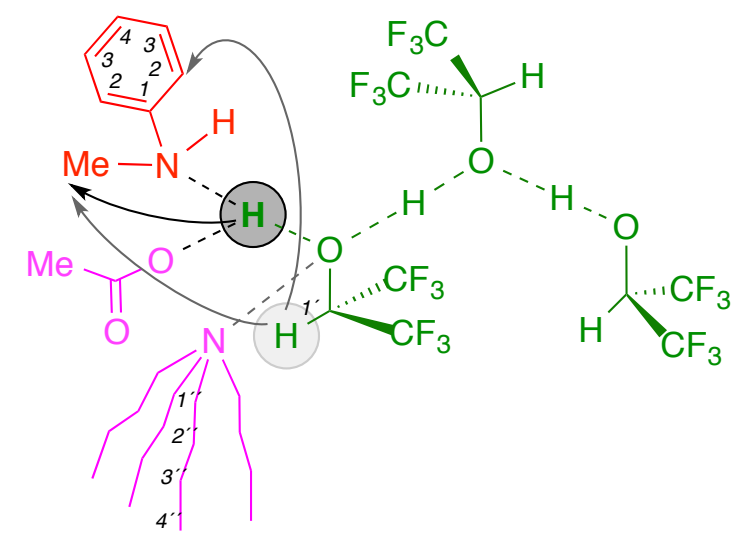

Finally, two small cross-peaks between the Me of the aniline and methylene units of the butyl chain $\left(2{ }^{\prime \prime}-\mathrm{H}\right.$ and $\left.3{ }^{\prime \prime}-\mathrm{H}\right)$ emerge from the baseline (red boxes in Figure S2). However, in this particular case these peak are near to other peaks belonging to the methylene coupling between $2^{\prime \prime}-\mathrm{H}$ and $3{ }^{\prime \prime}-\mathrm{H}$.

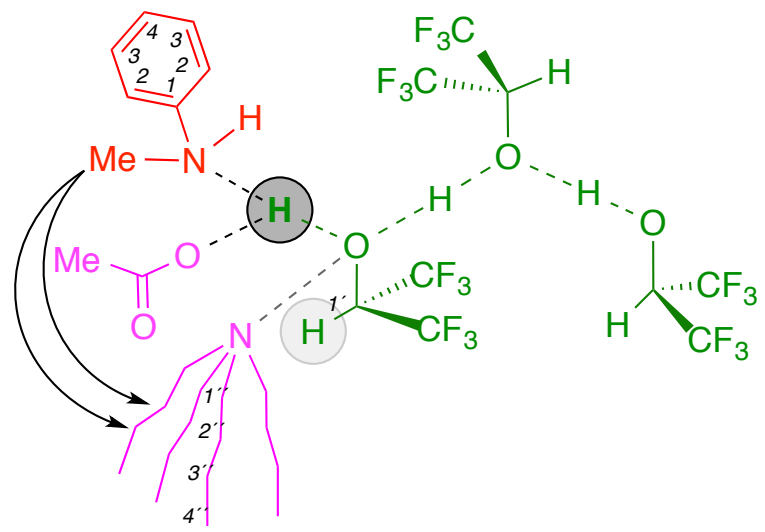




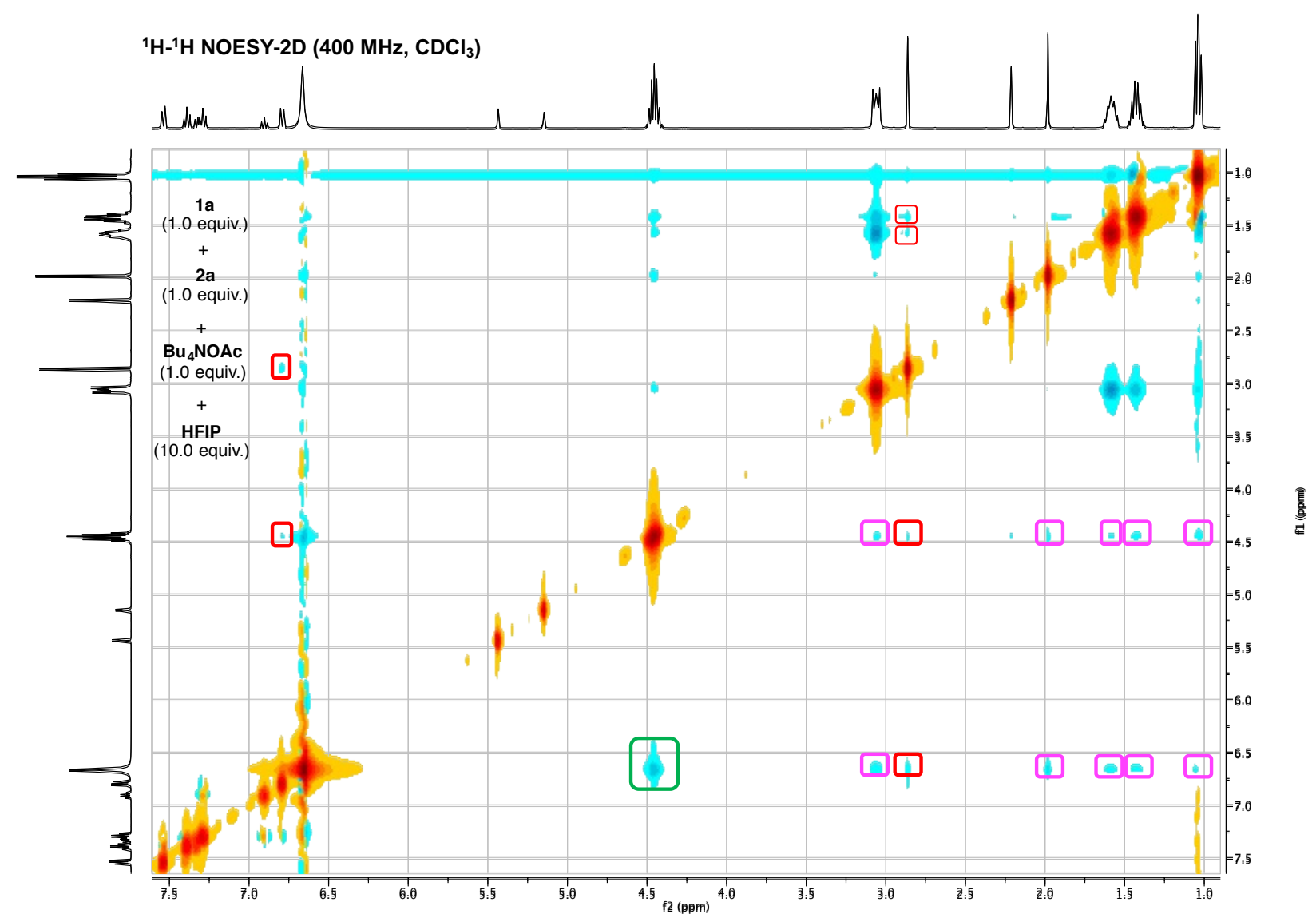

Figure S2: NOESY-2D of the mixture of aniline $1 a+$ alkene $2 a+B u_{4} N O A c+H F I P$.

\subsection{7. nOe-1D experiments}

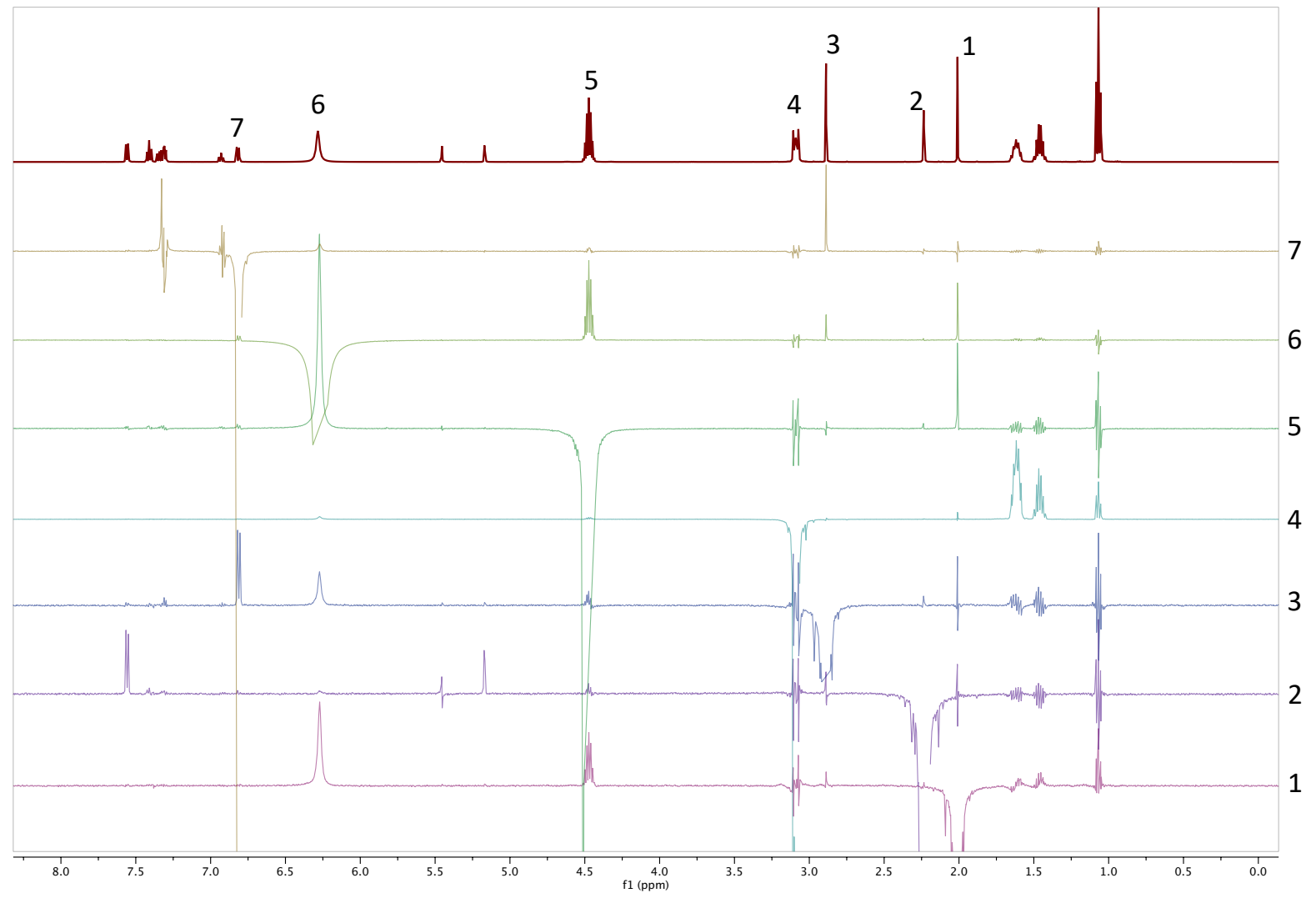

Figure S3: ${ }^{1} H$ NMR of the selective nOe irradiations. 
From the 1D nOe experiments (Figure S3), the same conclusion is extracted. The most interesting data is obtained by selectively irradiating the $\mathrm{OH}$ (Figure S4) and $\mathrm{CH}\left(1^{\prime}\right.$ - $\mathrm{H}$, Figure S5) of HFIP and the ortho-protons of the aniline (2-H, Figure S6).

When irradiating the $\mathrm{OH}$ nuclei a strong nOe effect is observed with $\mathrm{CH}\left(1^{\prime}-\mathrm{H}\right)$. Interestingly nOe effect with both ortho-aromatic protons of the aniline (2-H) and the N-Me of the anilne are also observed. Moreover, both the butyl aliphatic chain and the Me of the acetate gives significant nOe signals. These data confirm that both the $\mathrm{Bu}_{4} \mathrm{NOAc}$ and the aniline are connected to the HFIP.

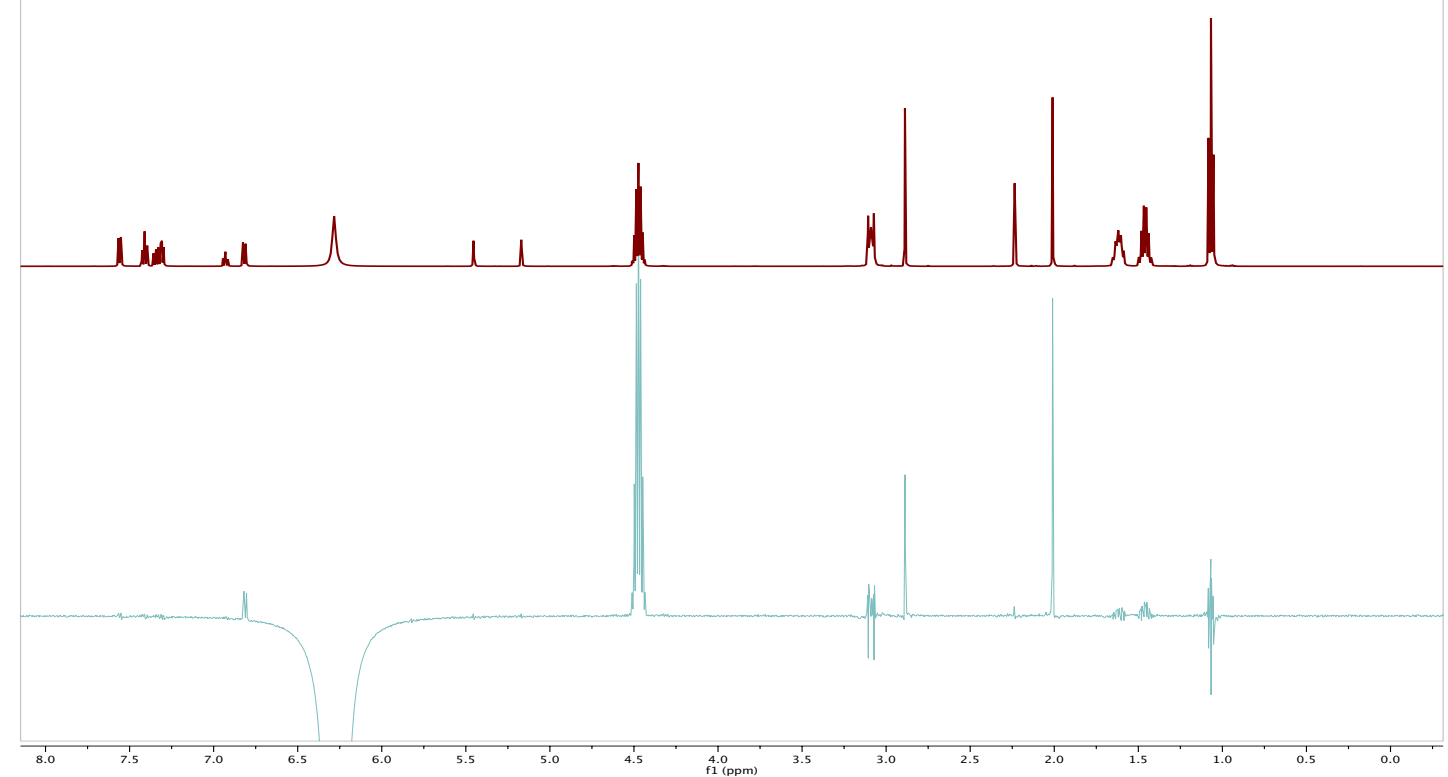

Figure S4: ${ }^{1} \mathrm{H}$ NMR and selective $\mathrm{OH}$ nOe irradiation.

When irradiating the $\mathrm{CH}\left(1^{\prime}-\mathrm{H}\right)$ nuclei a similar pattern is observed, although with diminished intensity of the signals, probably due to a longer distance.

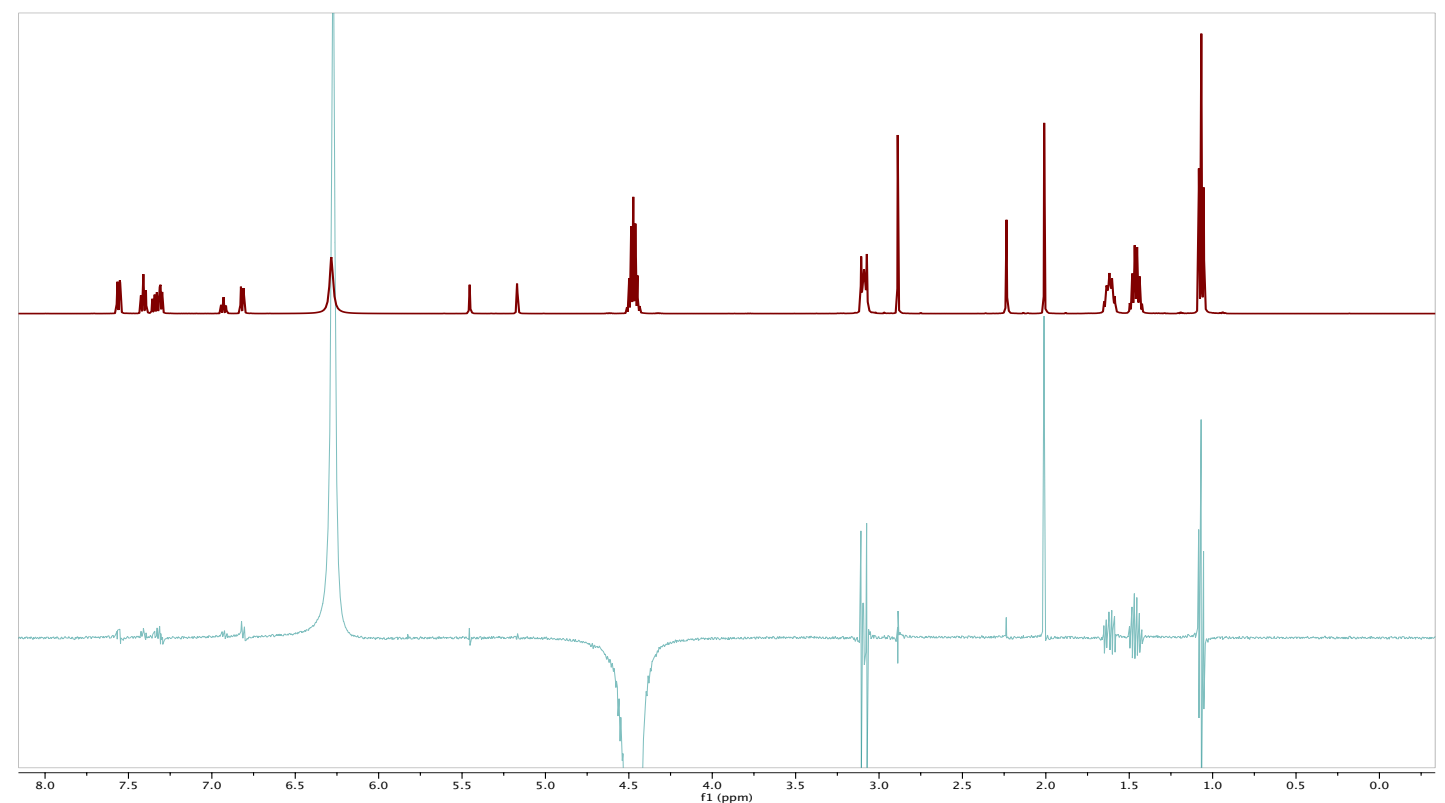

Figure S5: ${ }^{1} \mathrm{H} N \mathrm{~N} R$ and selective $\mathrm{CH}\left(1^{\prime}-H\right)$ nOe irradiation. 
Finally, when irradiating the ortho-aromatic protons of the aniline (2-H) nOe effect is observed with $\mathrm{OH}$ and $\mathrm{CH}\left(1^{\prime}-\mathrm{H}\right)$ of HFIP, and with the butyl chain and Me-OAc of $\mathrm{Bu}_{4} \mathrm{NOAc}$.

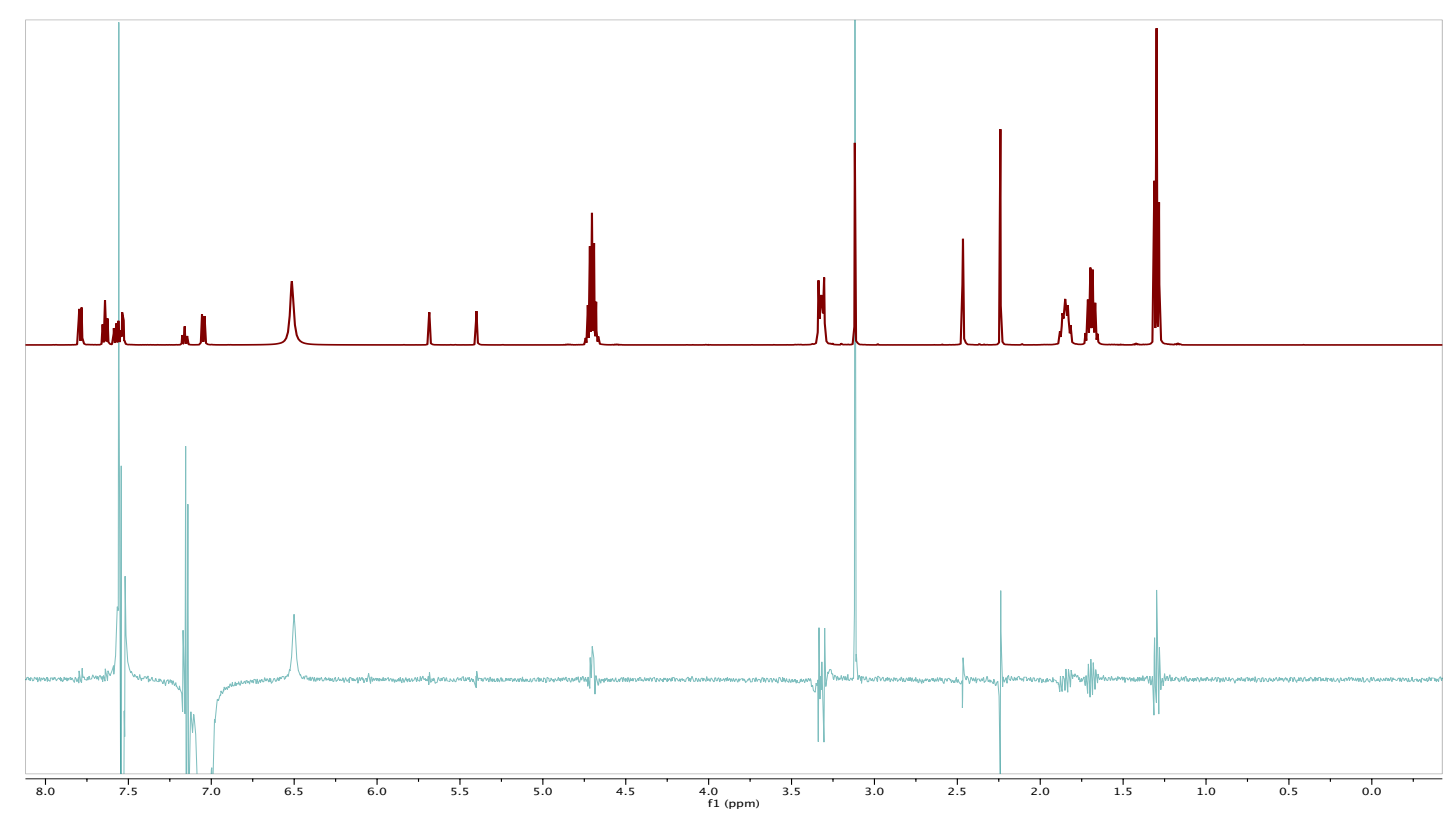

Figure S6: ${ }^{1} \mathrm{H}$ NMR and selective 2-H nOe irradiation.

\section{References}

${ }^{1}$ Budai, B.; Leclair, A.; Wang, Q.; Zhu, J., Copper-Catalyzed 1,2-Methoxy Methoxycarbonylation of Alkenes with Methyl Formate. Angew. Chem. Int. Ed. 2019, 58, 10305-10309.

${ }^{2}$ Cabré, A.; Sciortino, G.; Ujaque, G.; Verdaguer, X.; Lledós, A.; Riera, A., Iridium-Catalyzed Isomerization of N-Sulfonyl Aziridines to Allyl Amines. Org. Lett. 2018, 20, 5747-5751.

${ }^{3}$ Phan, D. H. T.; Kou, K. G. M.; Dong, V. M., Enantioselective Desymmetrization of Cyclopropenes by Hydroacylation. J. Am. Chem. Soc. 2010, 132, 16354-16355. 


\section{NMR spectra}

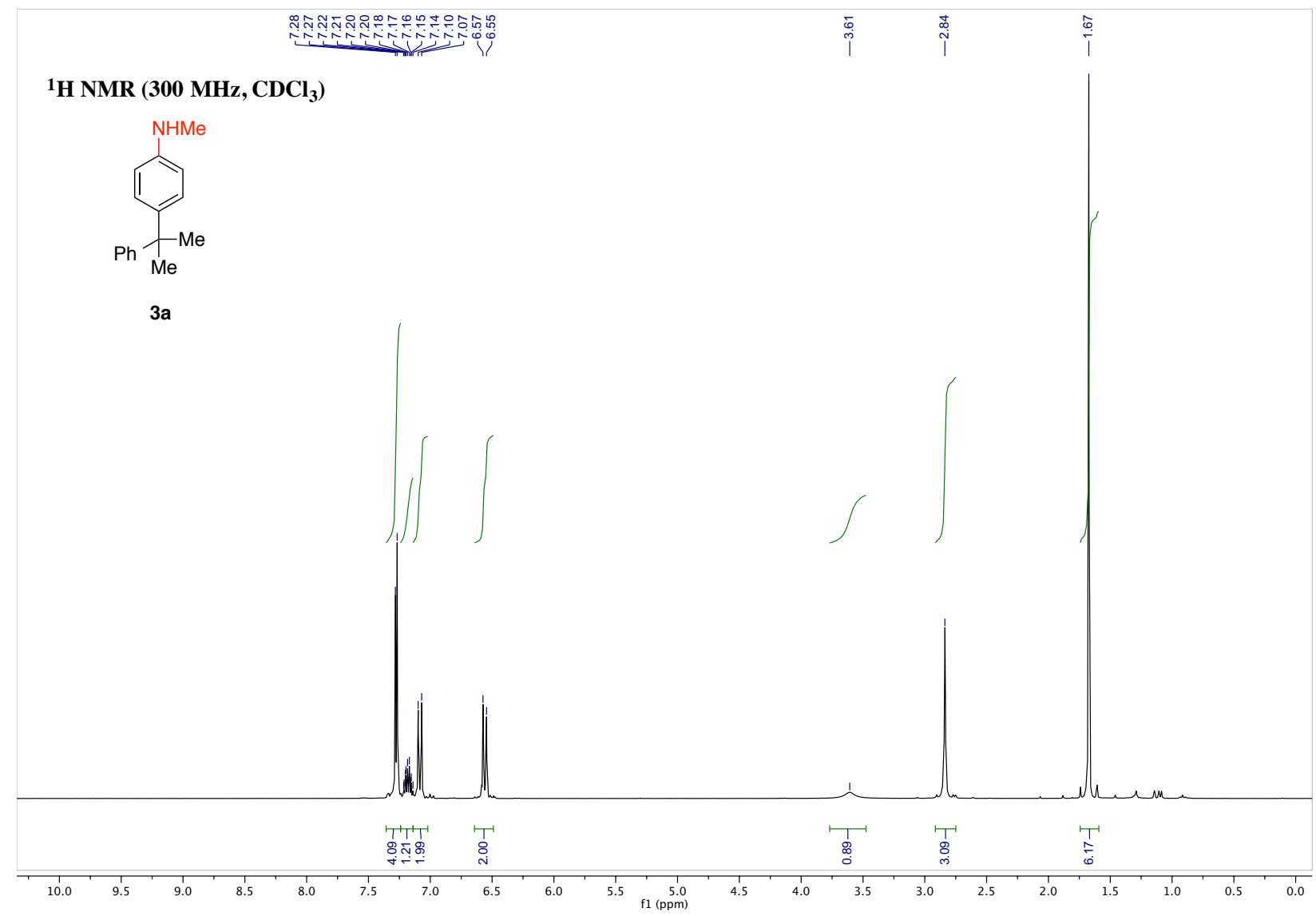

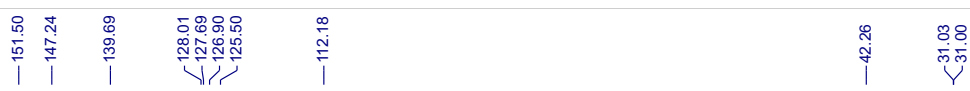

${ }^{13} \mathrm{C}$ NMR (75 MHz, $\left.\mathrm{CDCl}_{3}\right)$

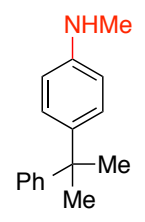

3a
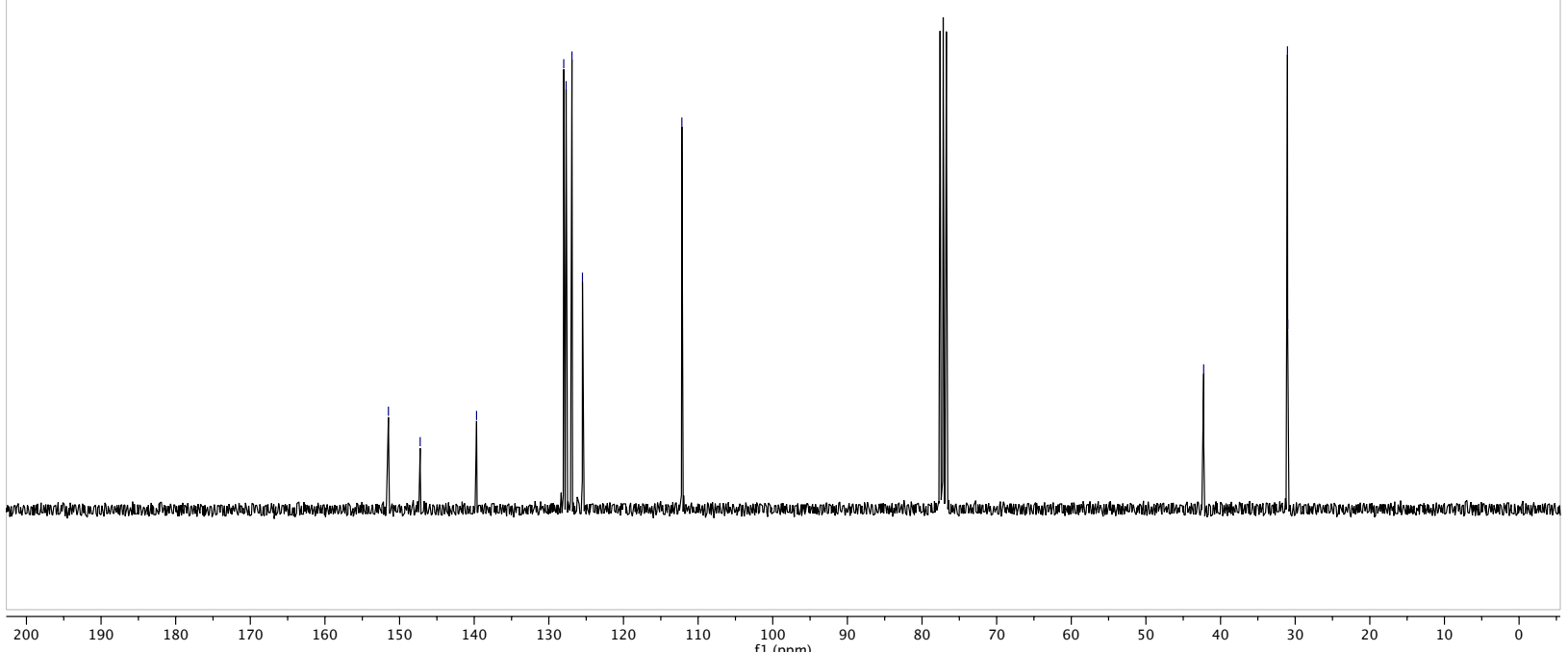
S40
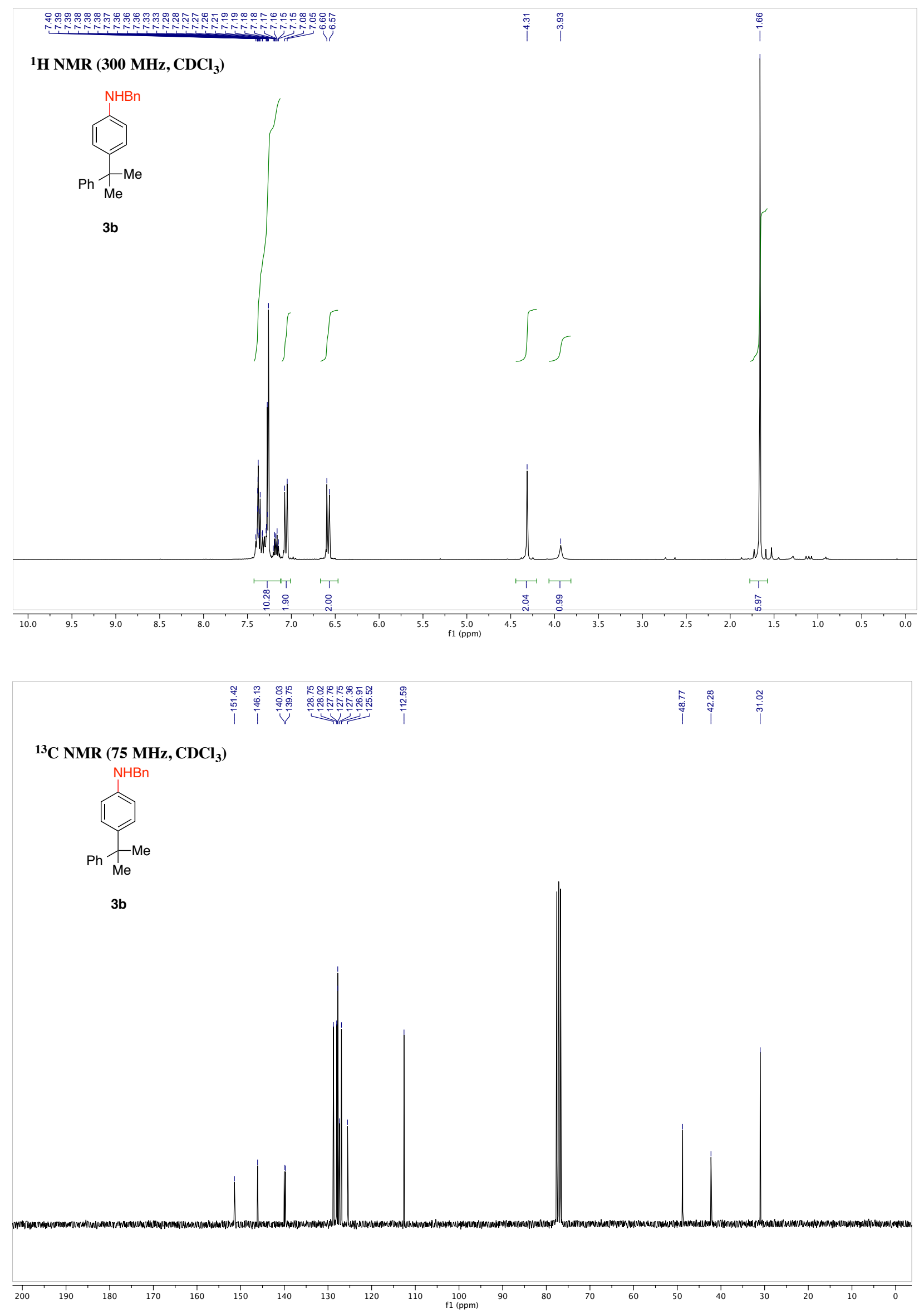

S40 

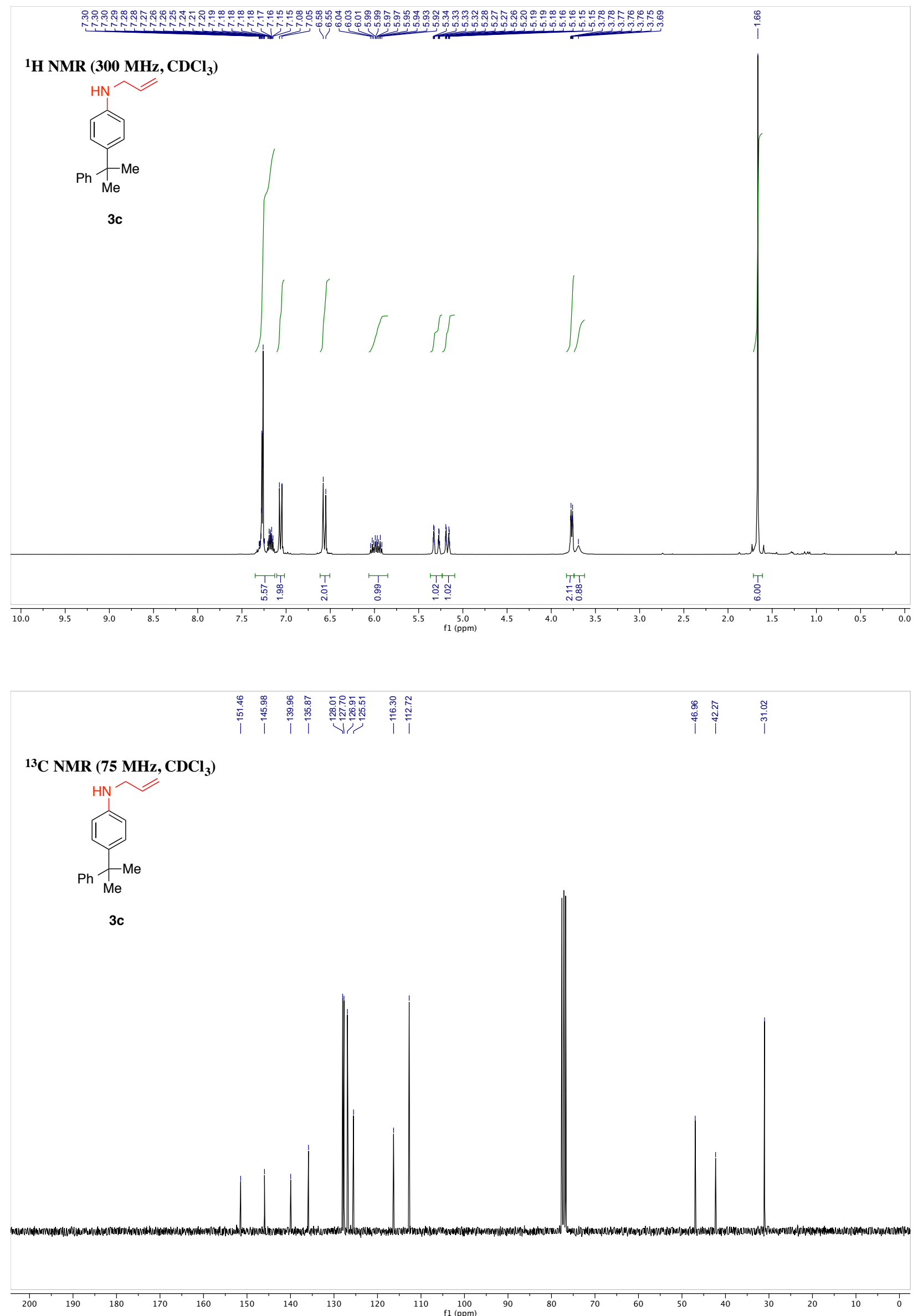


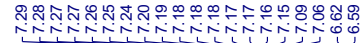

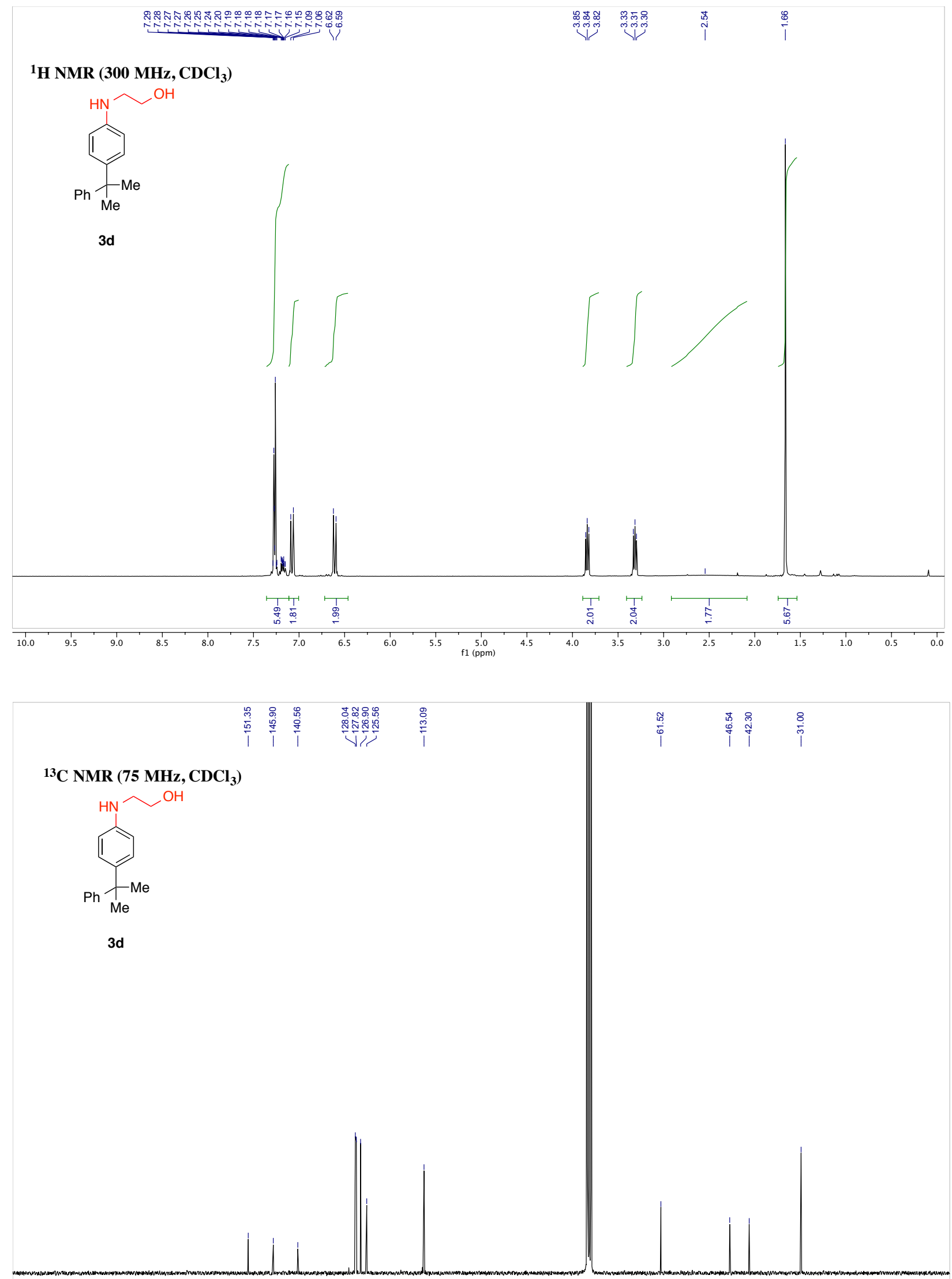

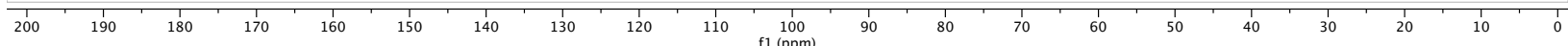




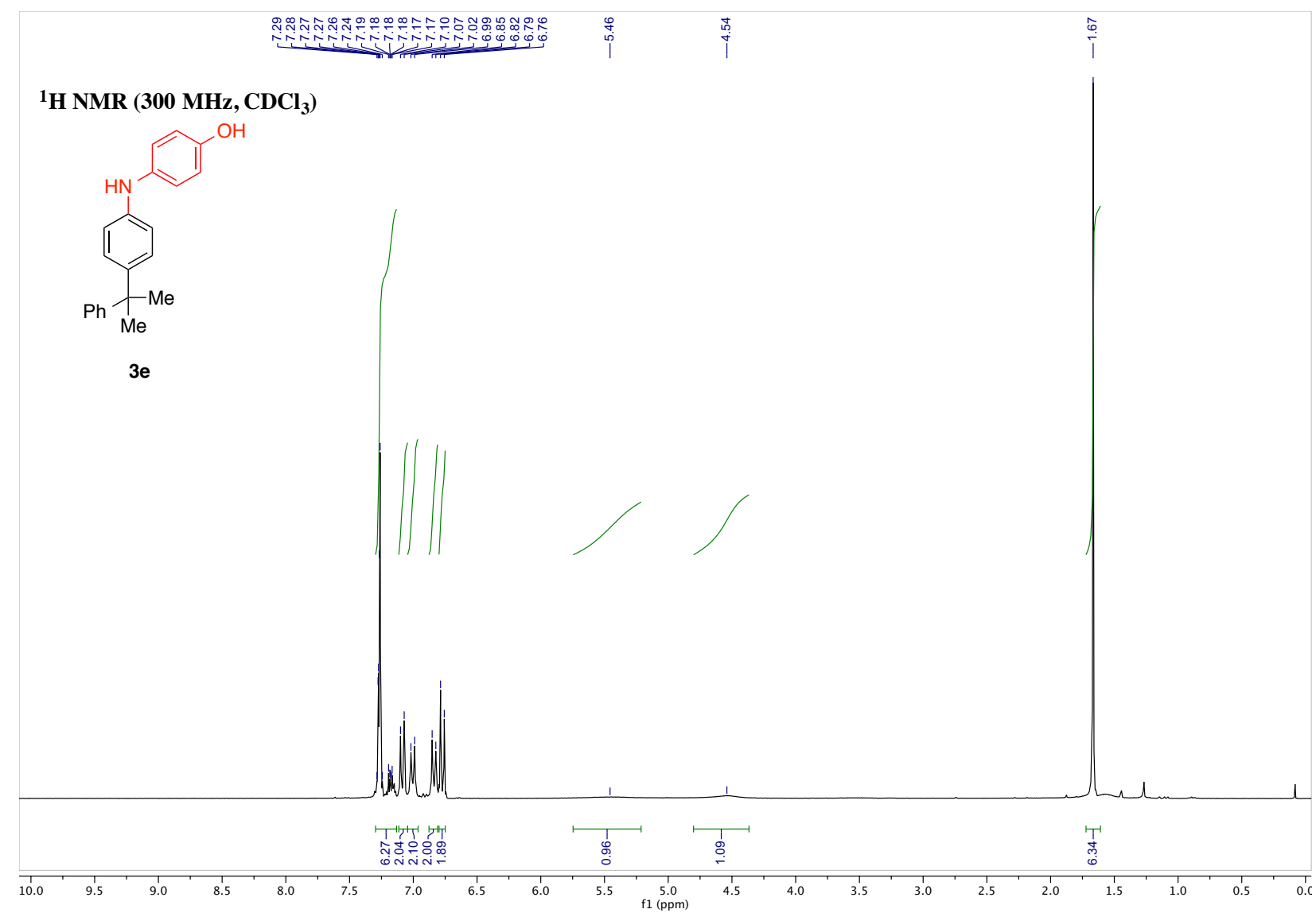

${ }^{13} \mathrm{C}$ NMR (75 MHz, $\left.\mathrm{CDCl}_{3}\right)$

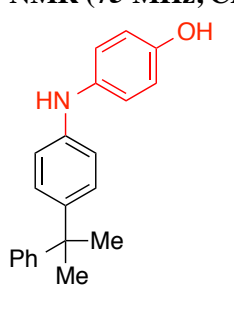

$3 e$

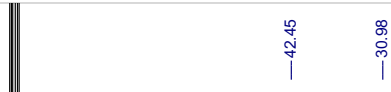

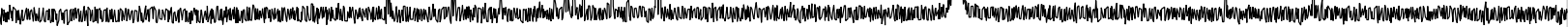

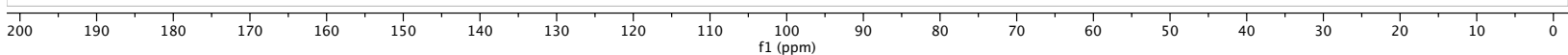



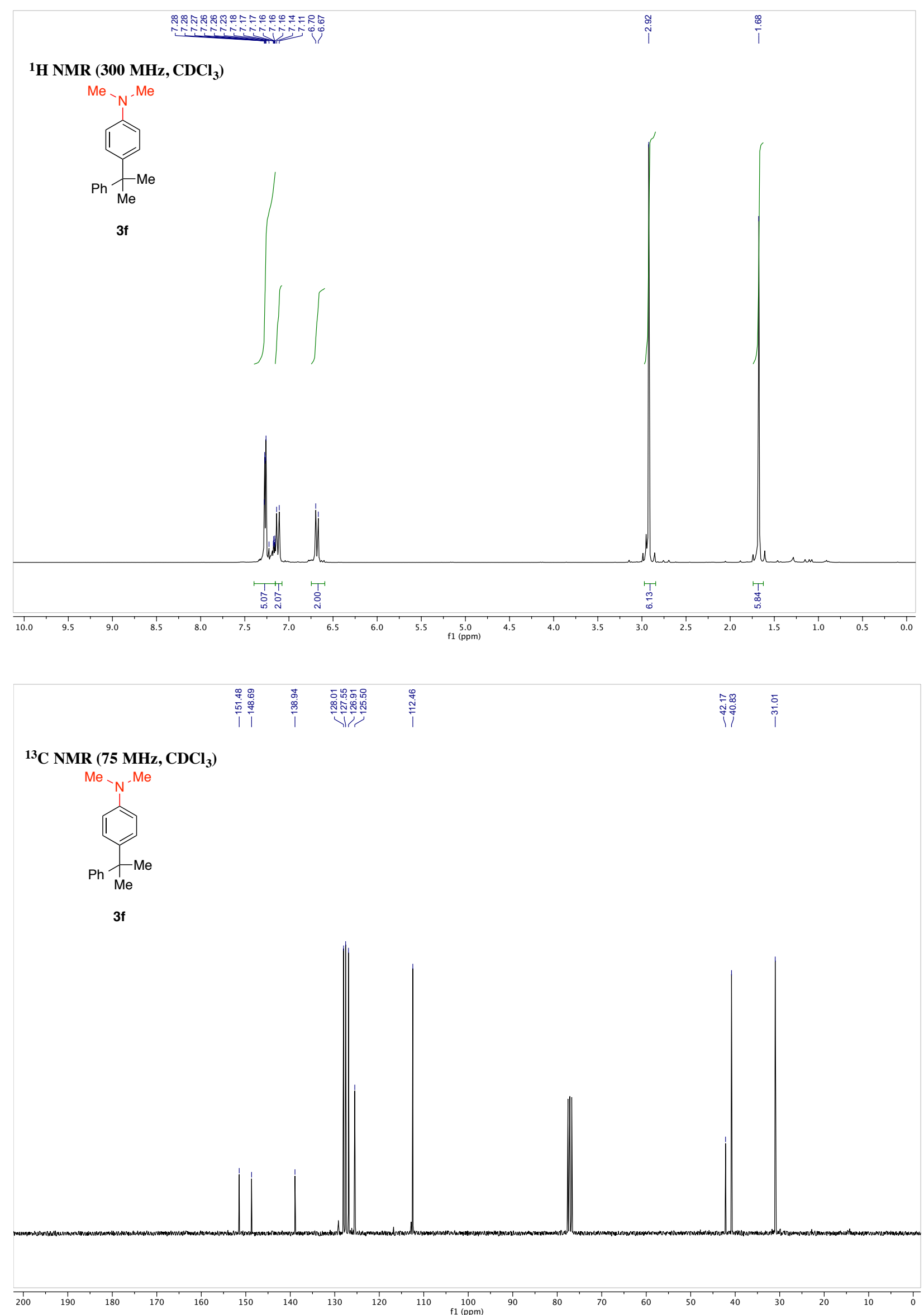


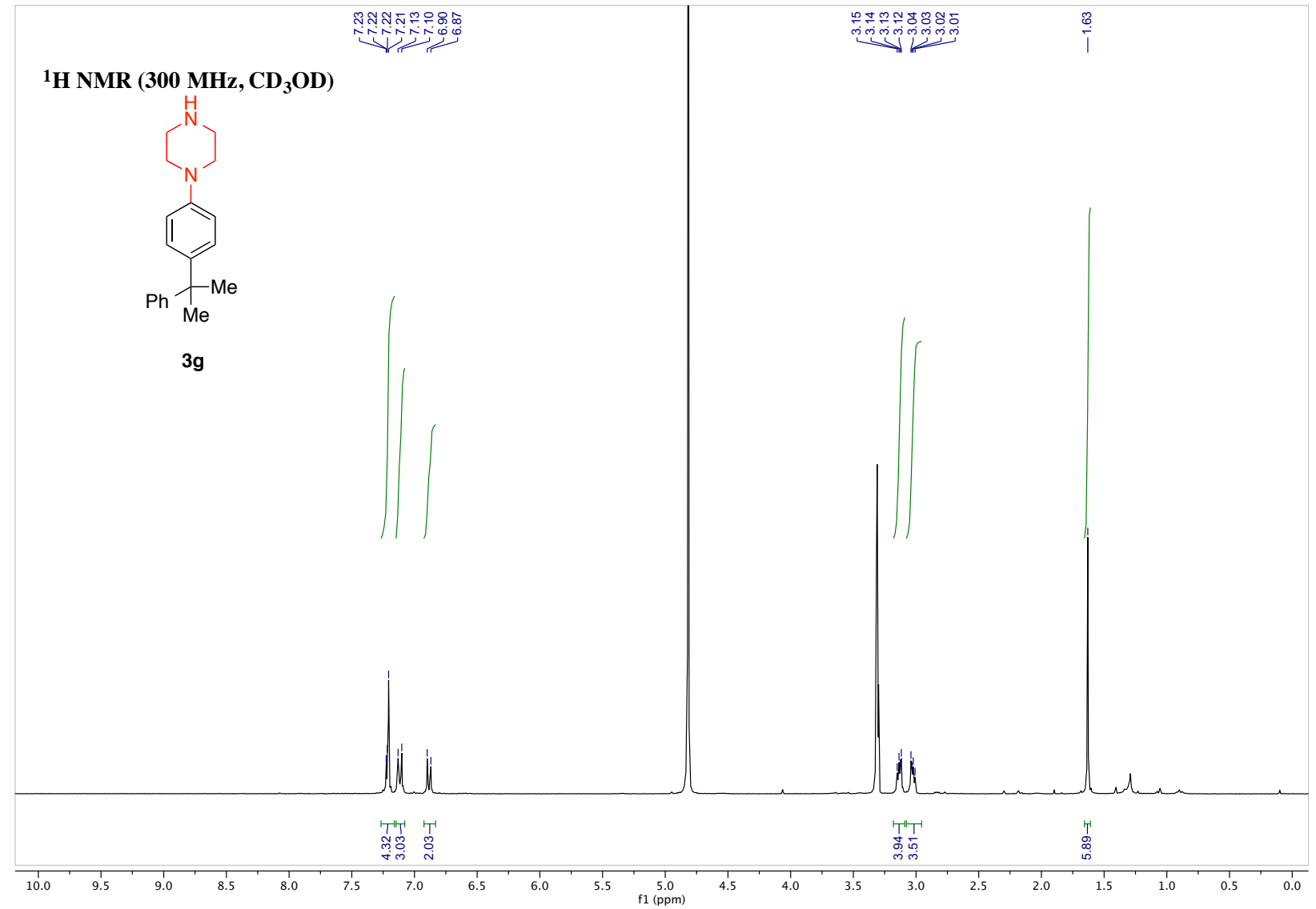

${ }^{13} \mathrm{C}$ NMR (75 MHz, CD 3 OD)

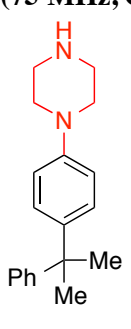

$3 g$
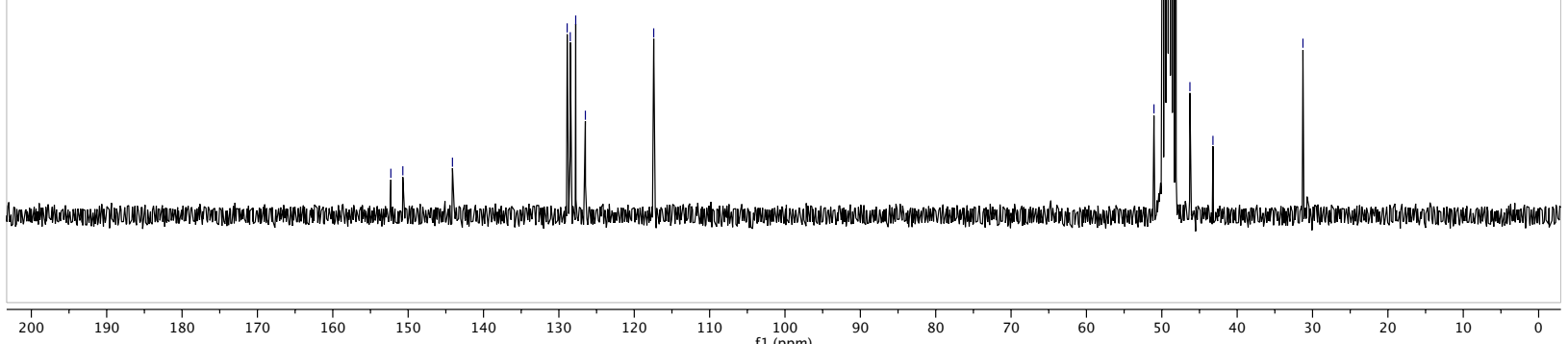
S46

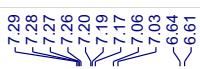

$\stackrel{\substack{0 \\ j}}{i}$

$\stackrel{\bullet}{i}$

${ }^{1} \mathrm{H}$ NMR (300 MHz, $\left.\mathrm{CDCl}_{3}\right)$

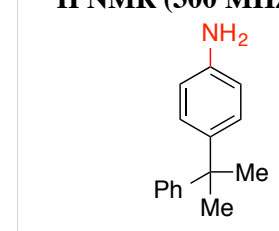

$3 h$
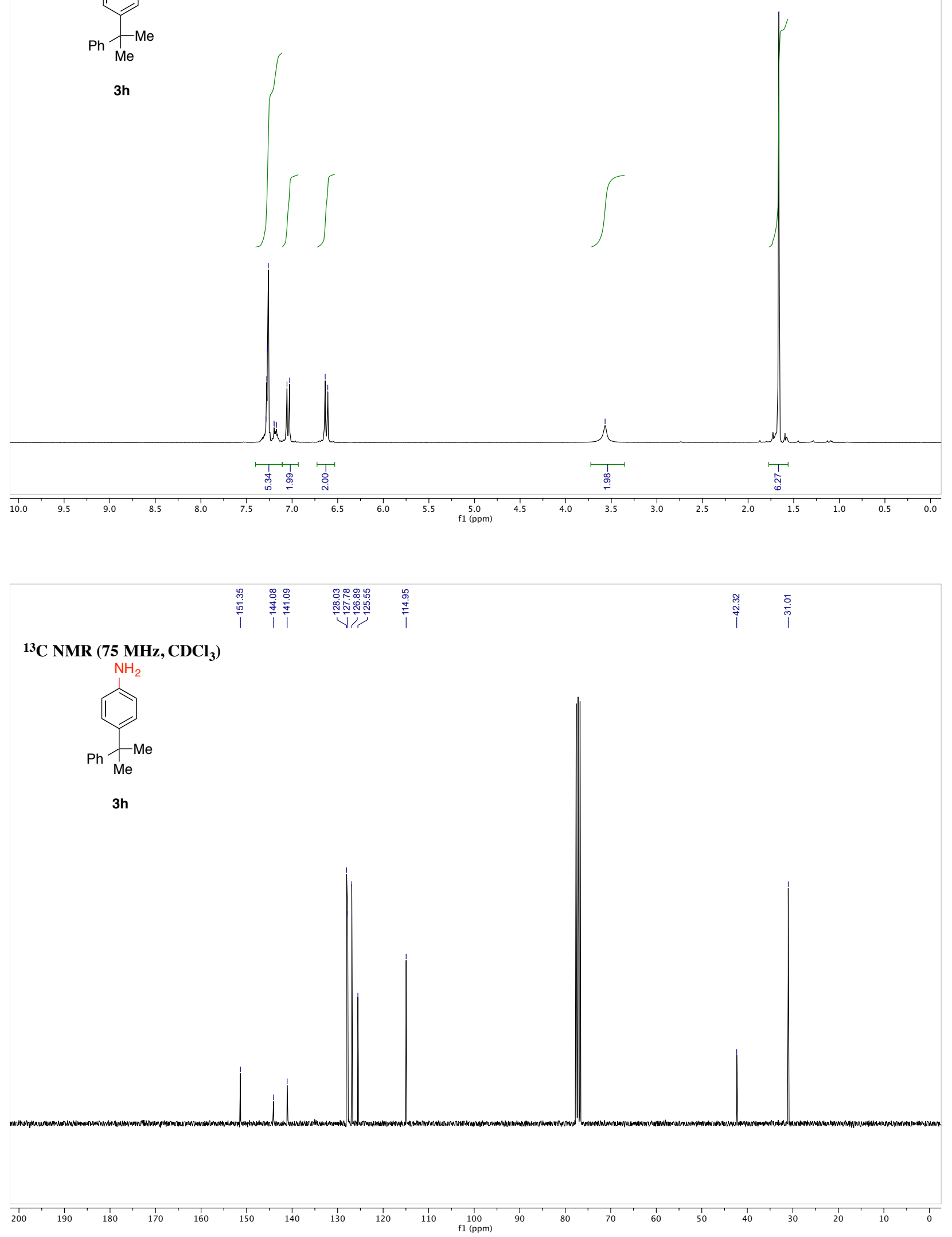

S46 
${ }^{1} \mathrm{H}$ NMR (300 MHz, $\left.\mathrm{CDCl}_{3}\right)$

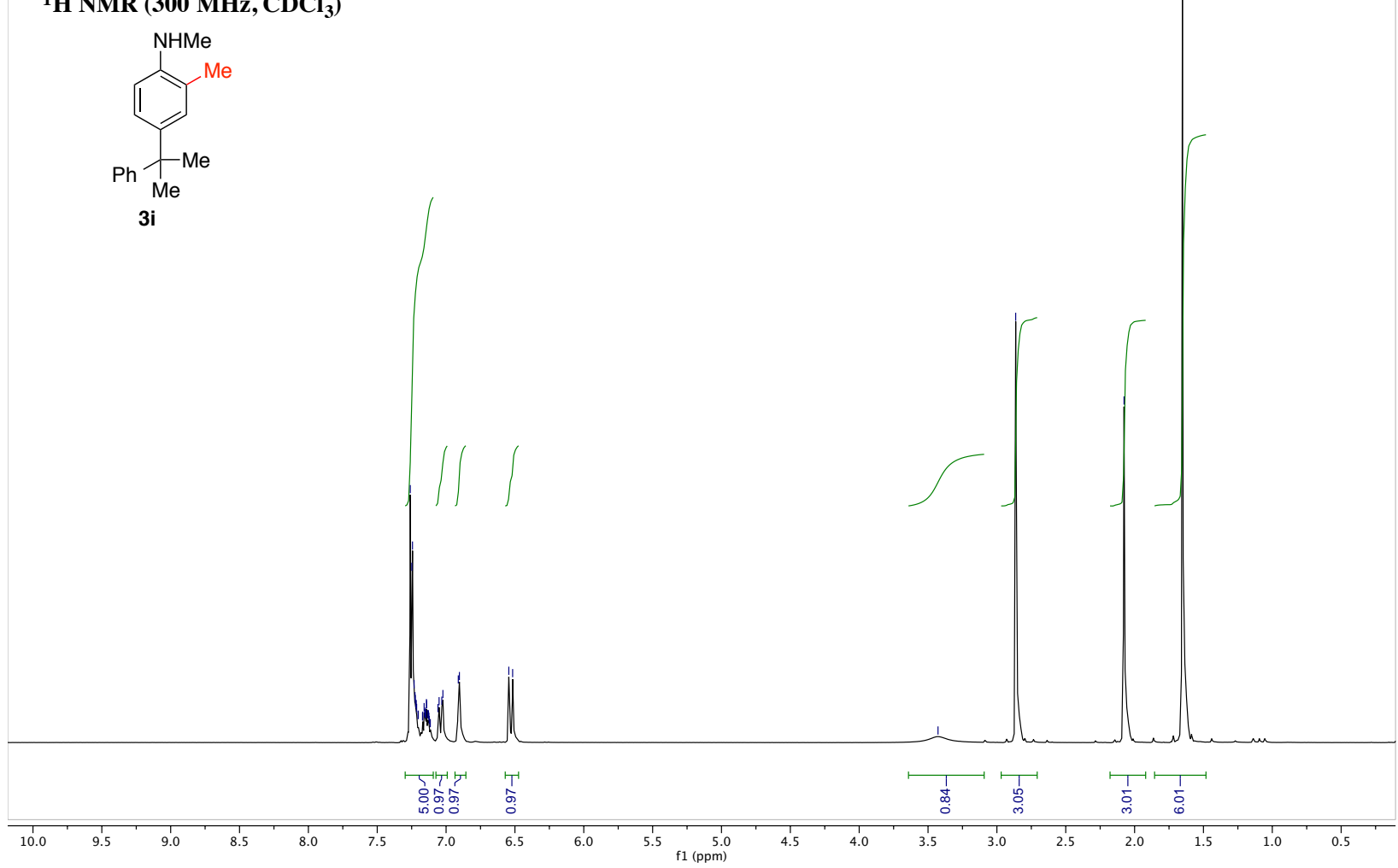

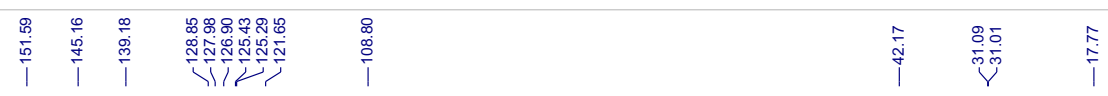

${ }^{13} \mathrm{C}$ NMR (75 $\left.\mathrm{MHz}, \mathrm{CDCl}_{3}\right)$

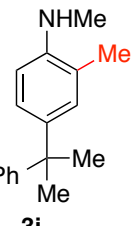

3i

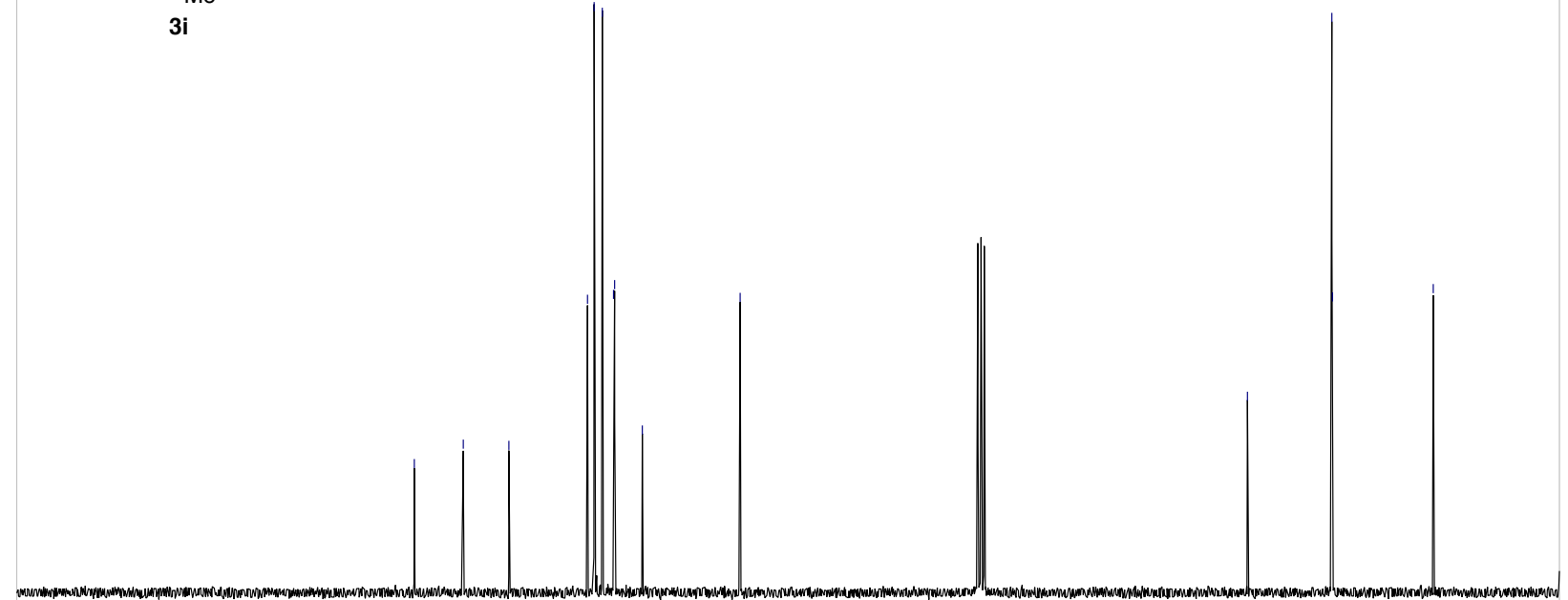

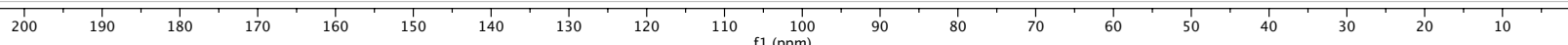



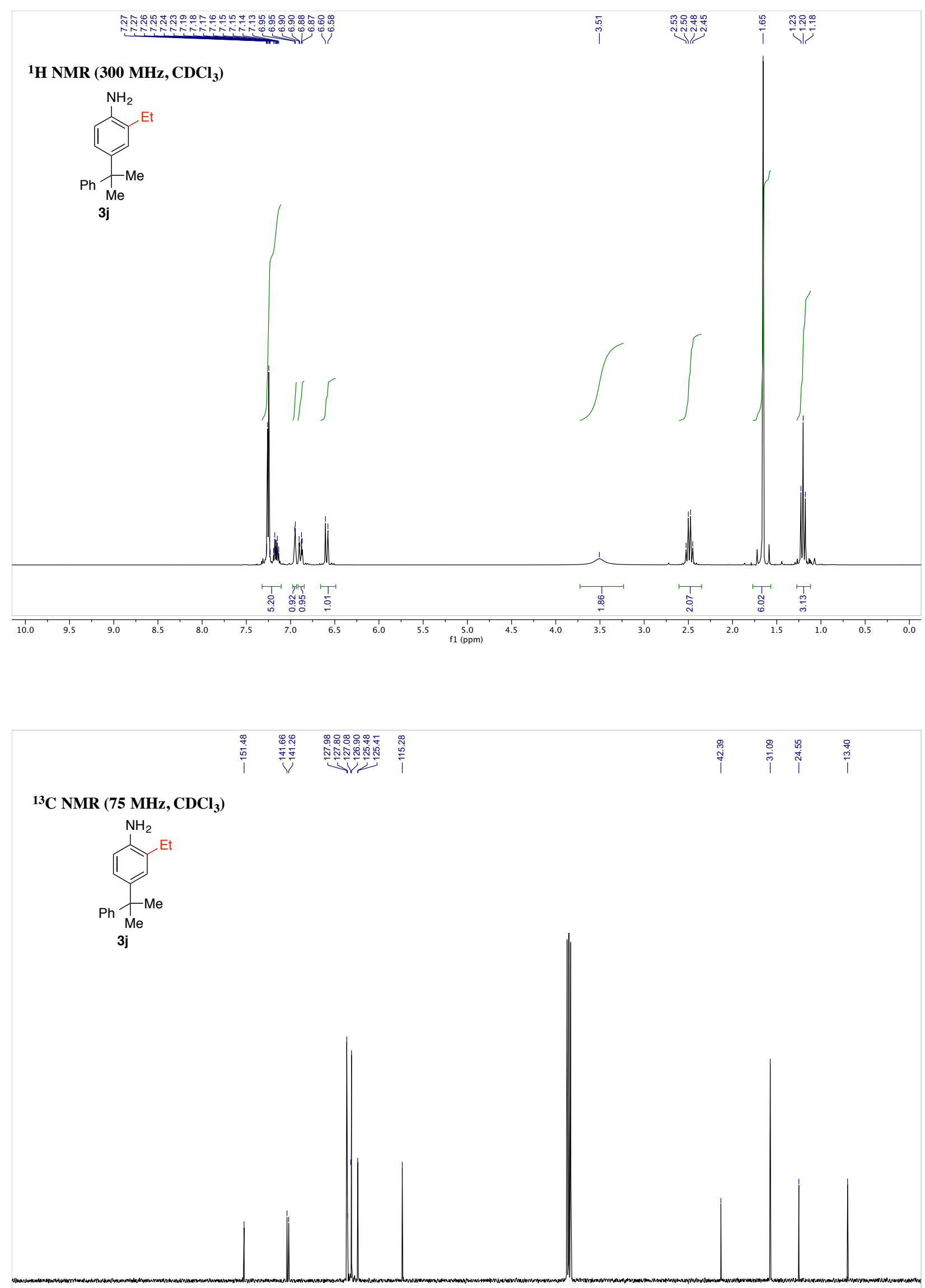

200
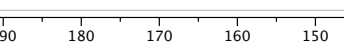

\| 


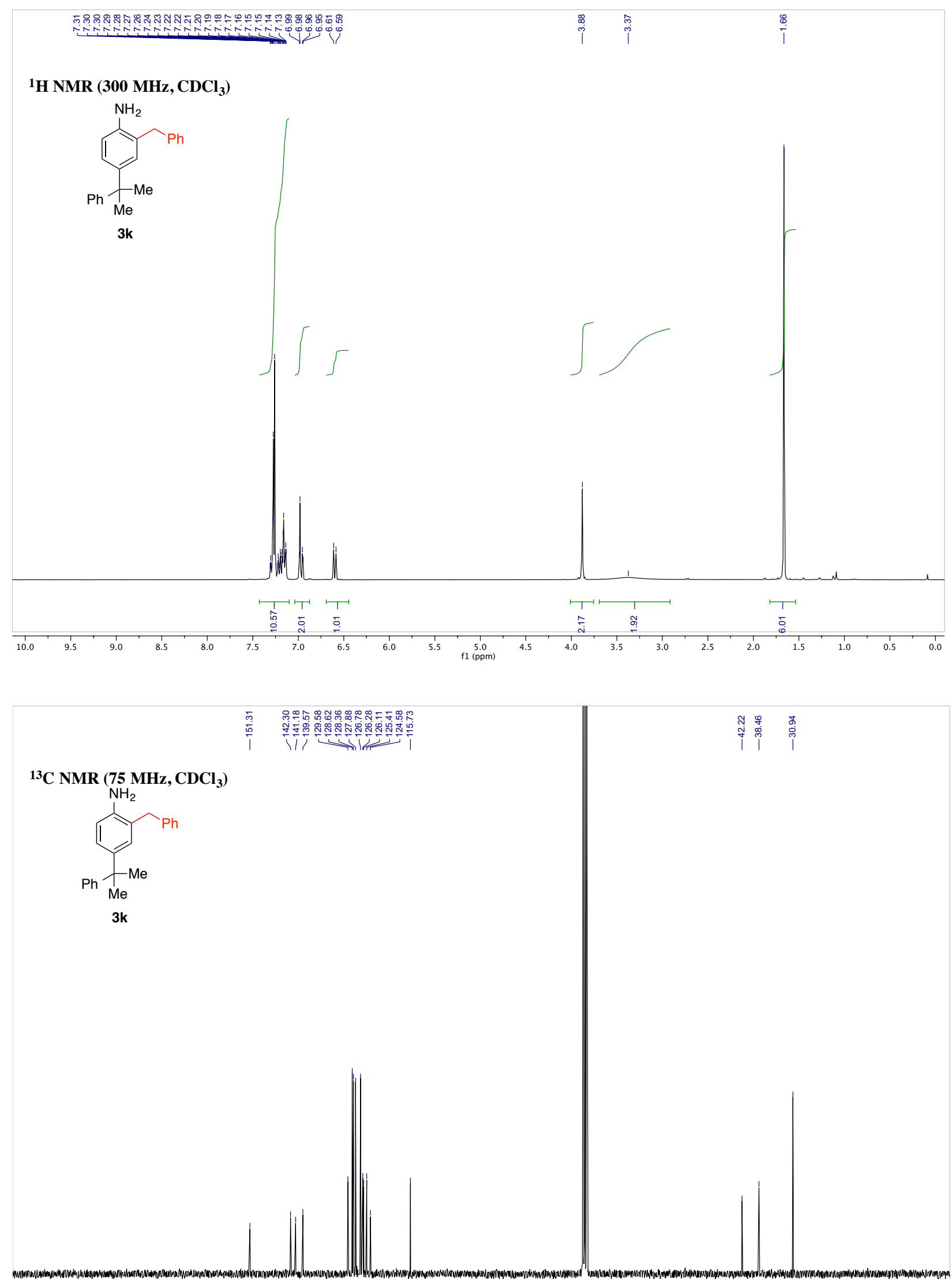

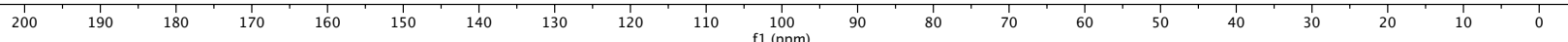




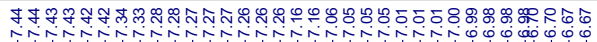

i

${ }^{1} \mathrm{H}$ NMR (300 MHz, $\left.\mathrm{CDCl}_{3}\right)$

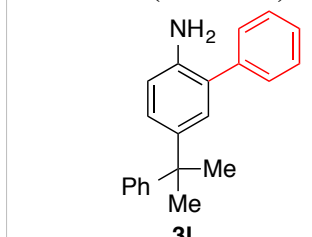

3I

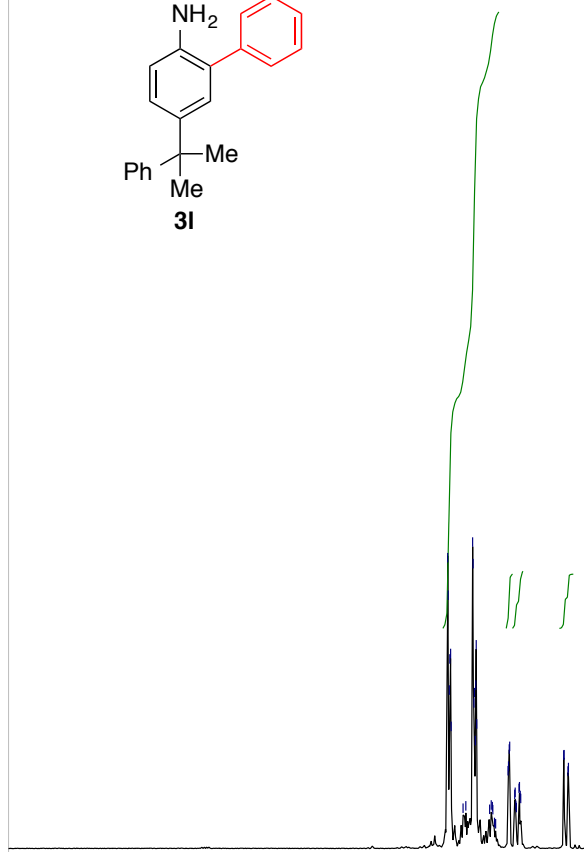

Win
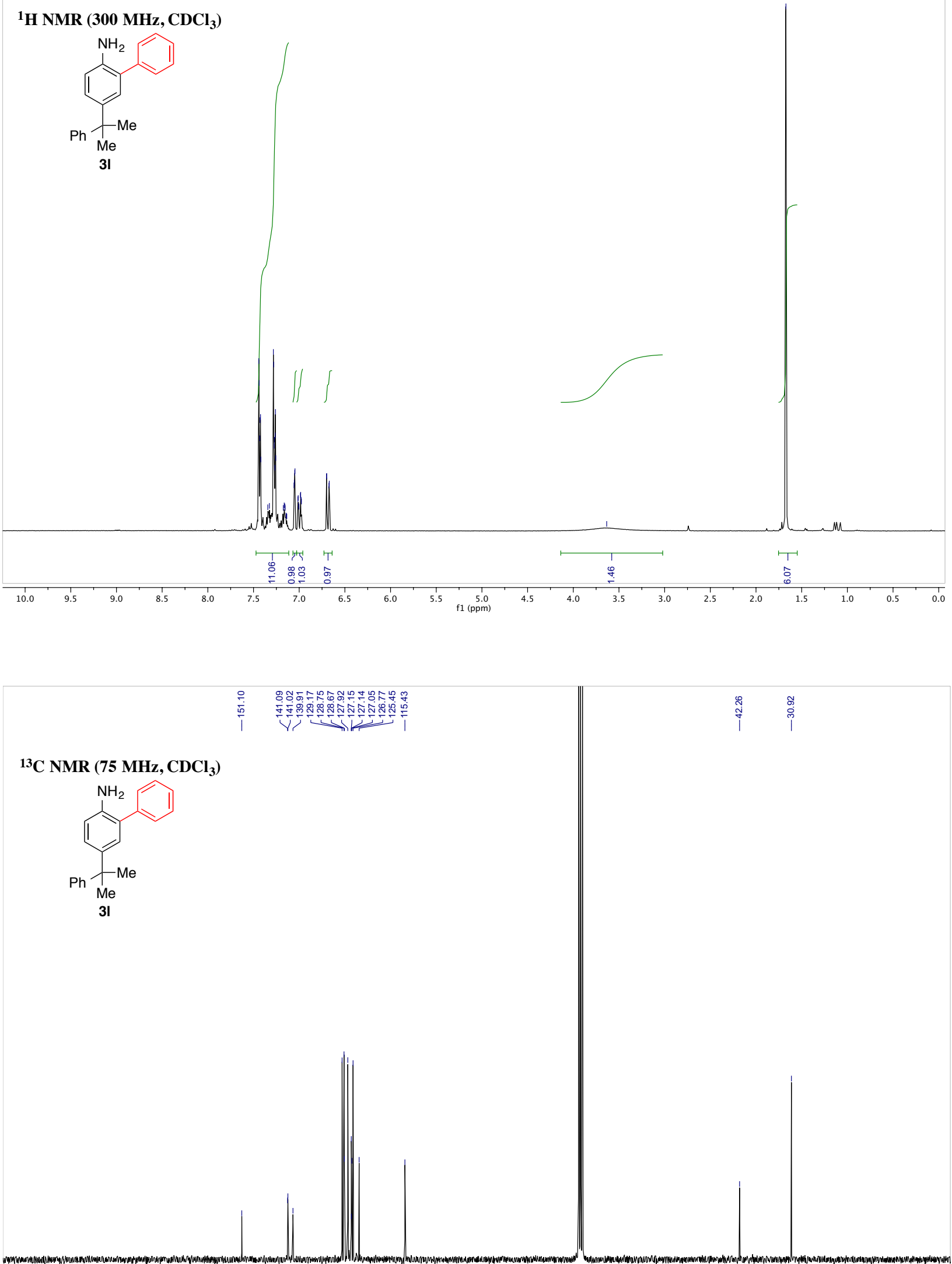

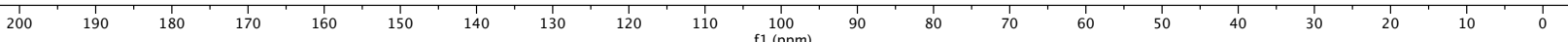




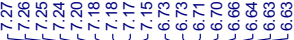

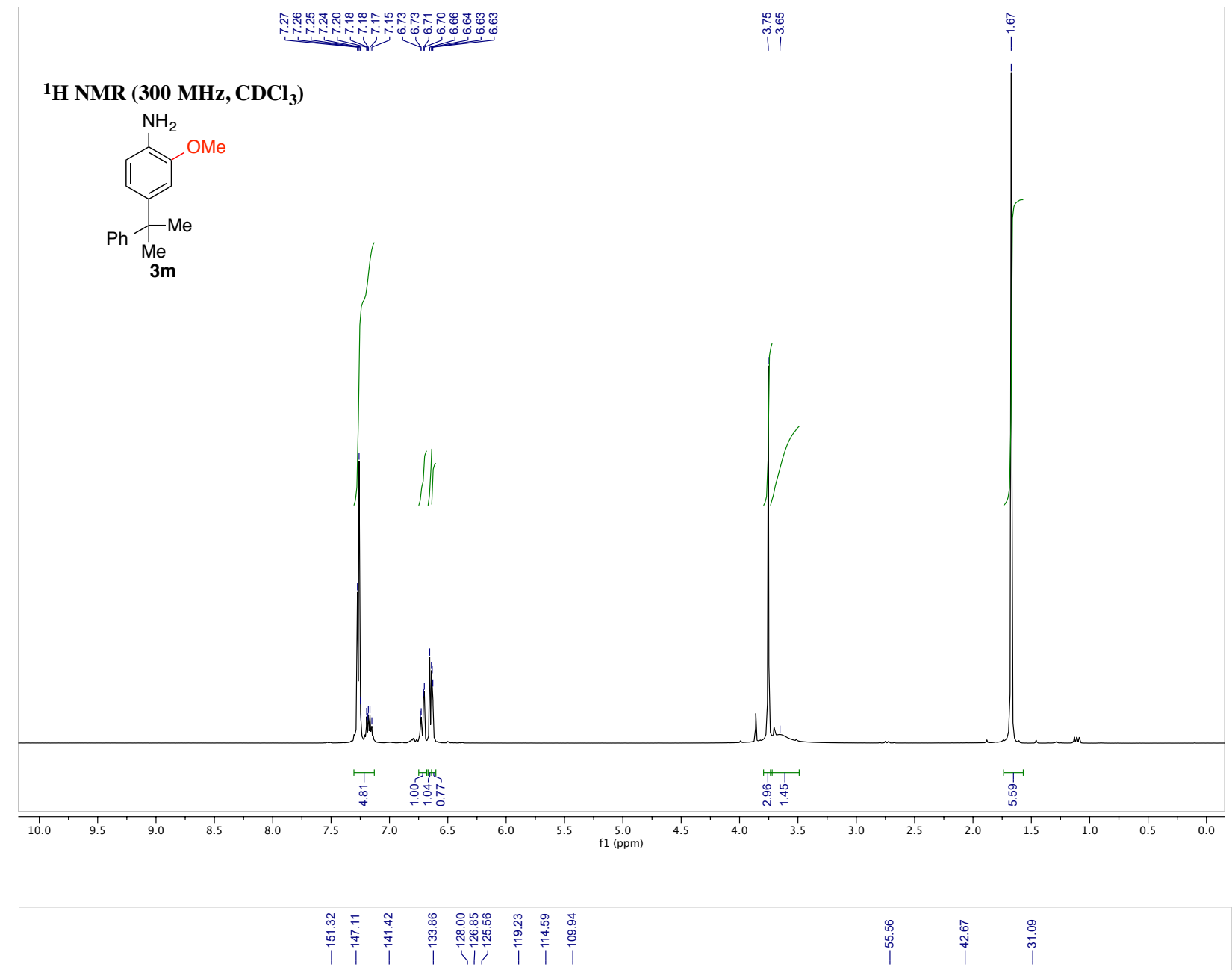

${ }^{13} \mathrm{C}$ NMR (75 $\left.\mathrm{MHz}, \mathrm{CDCl}_{3}\right)$

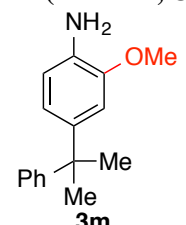

$3 \mathrm{~m}$

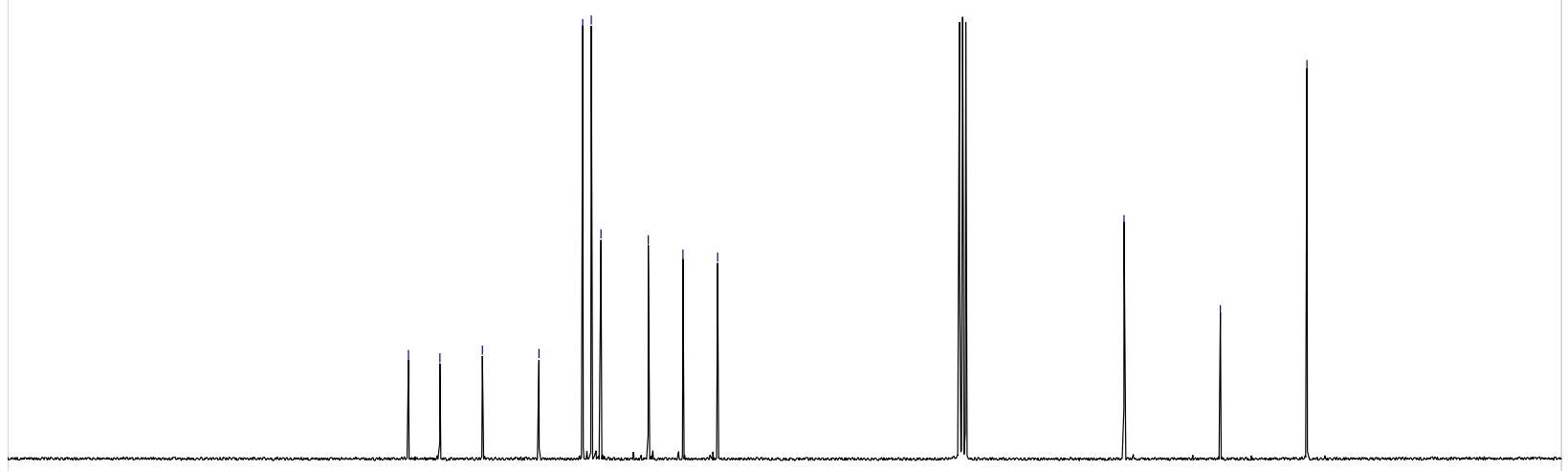

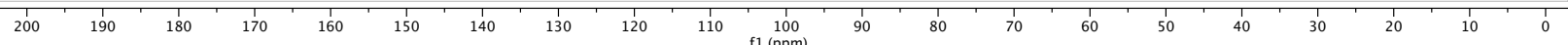


S52
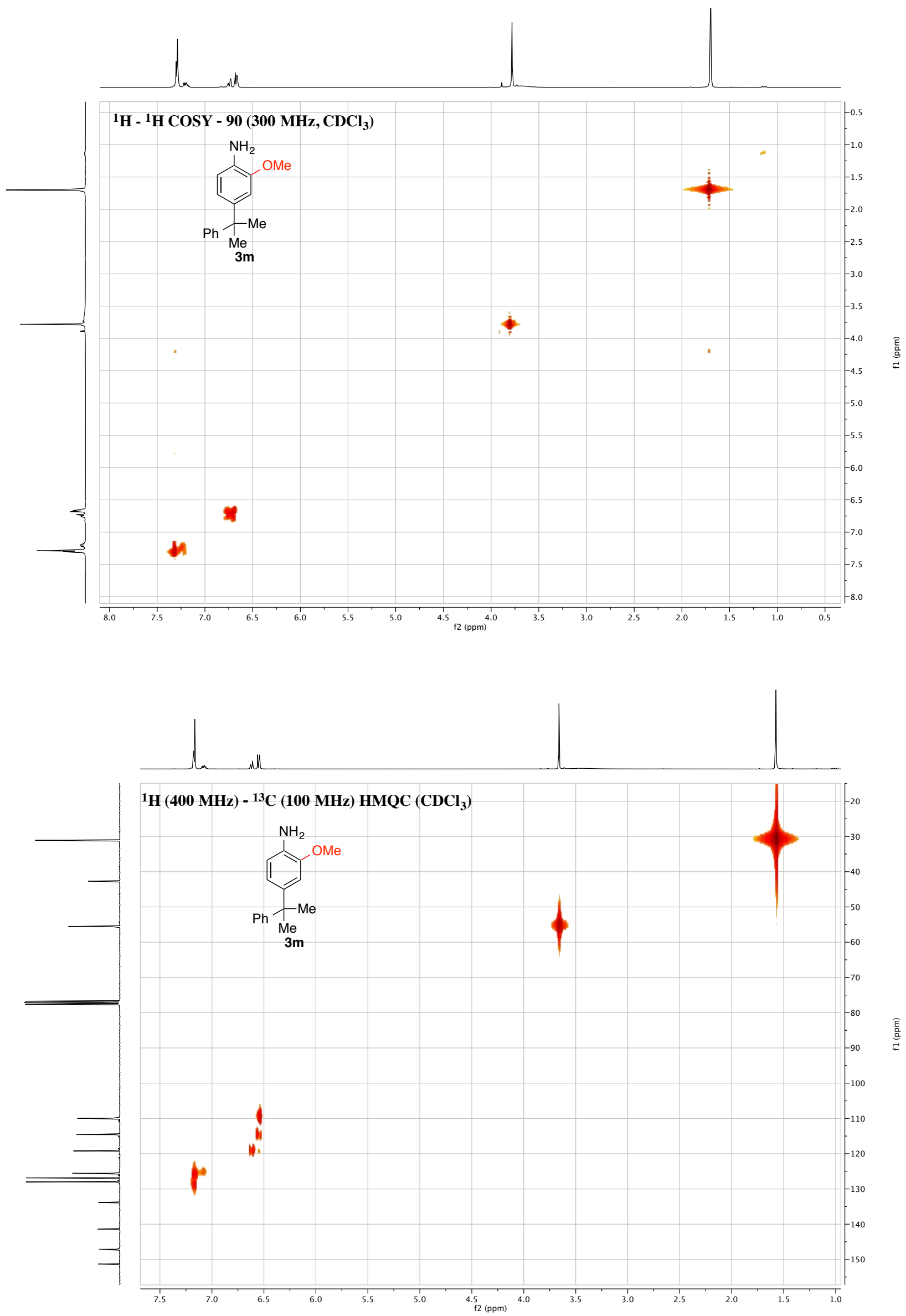

S52 

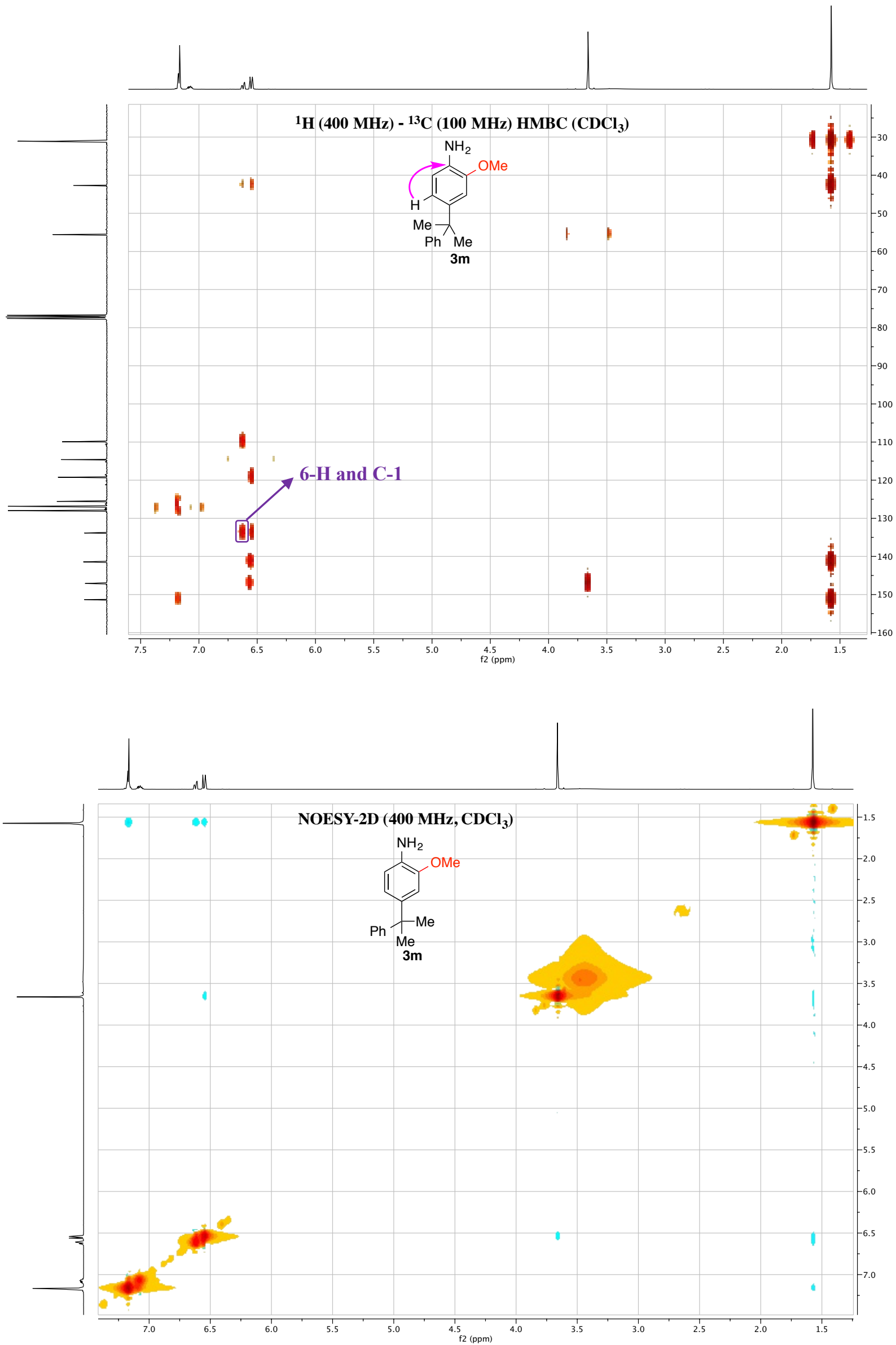


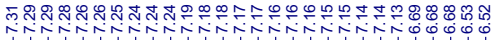

${ }^{1} \mathrm{H}$ NMR (300 MHz, $\left.\mathrm{CDCl}_{3}\right)$

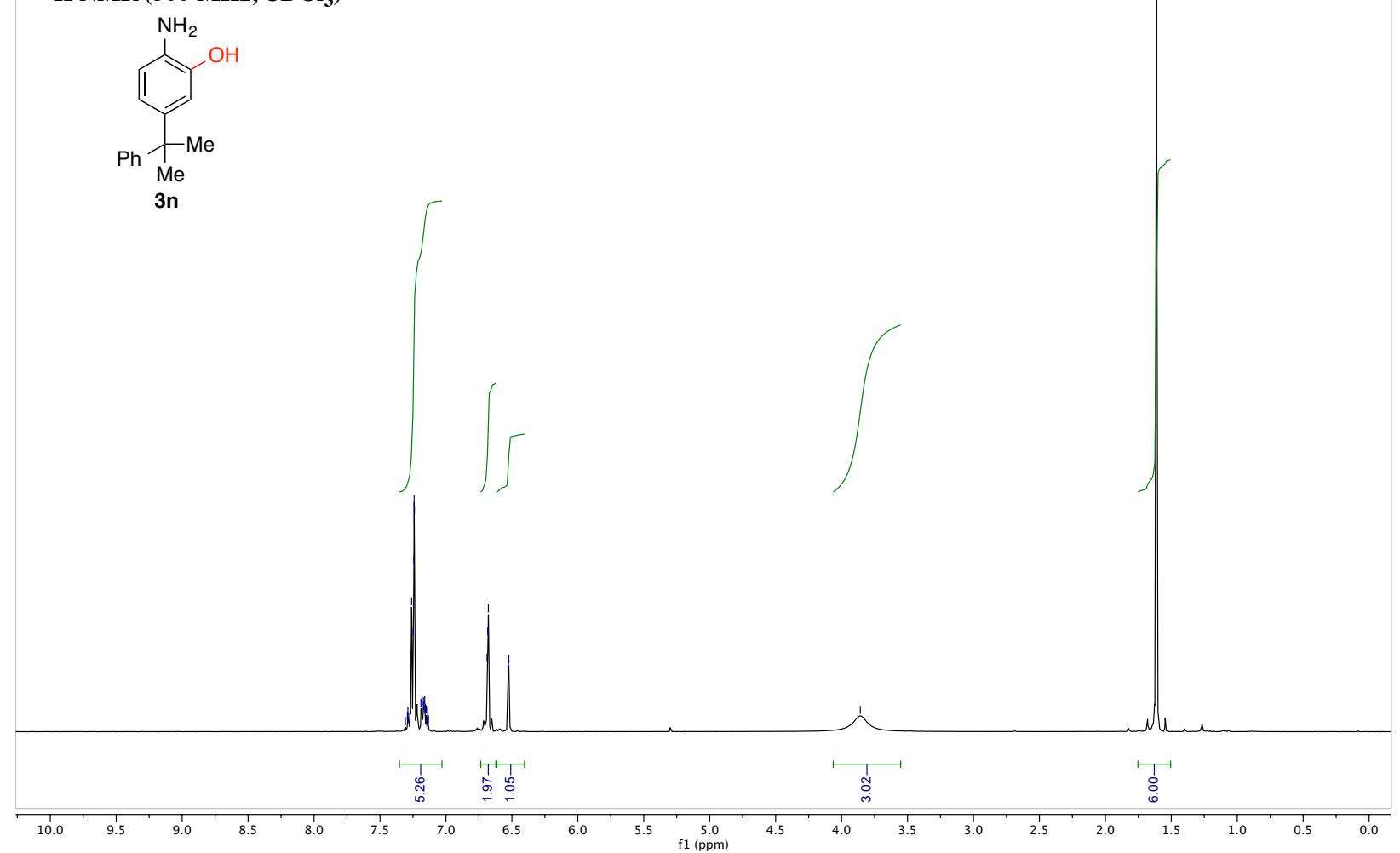

${ }^{13} \mathrm{C}$ NMR (75 $\mathrm{MHz}, \mathrm{CDCl}_{3}$ )

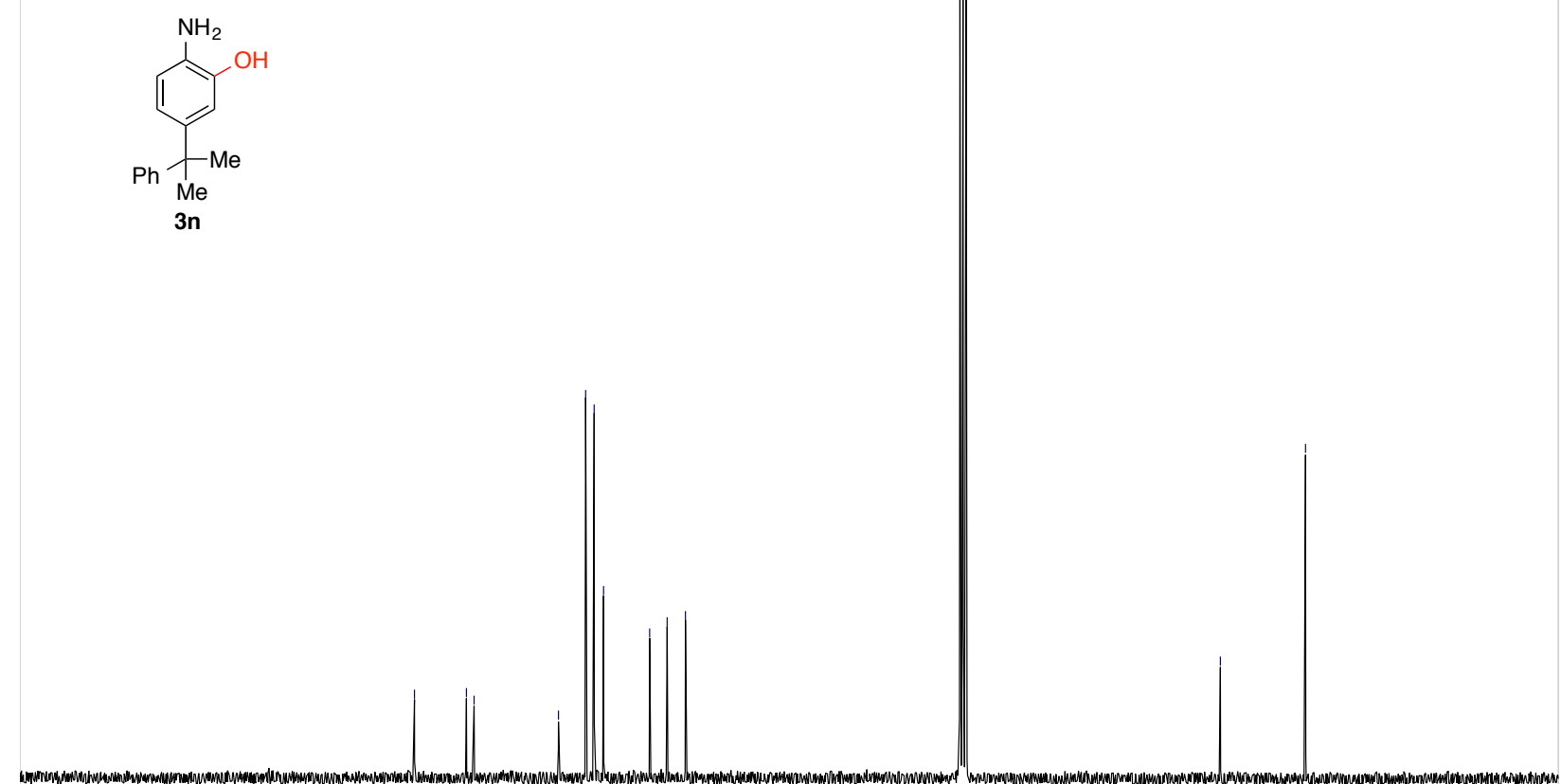

$3 n$

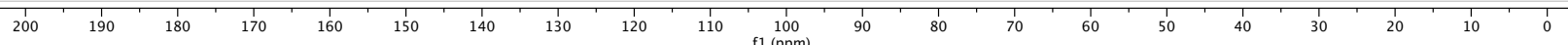




$$
\frac{I}{1}
$$




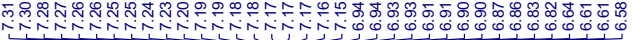

${ }^{1} \mathrm{H}$ NMR (300 MHz, $\left.\mathrm{CDCl}_{3}\right)$

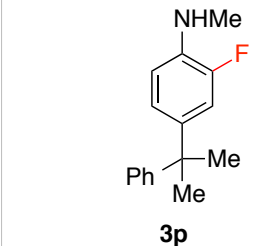

$3 p$

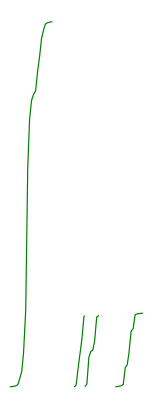

州

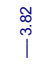

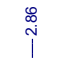

$\stackrel{\leftrightarrow}{i}$

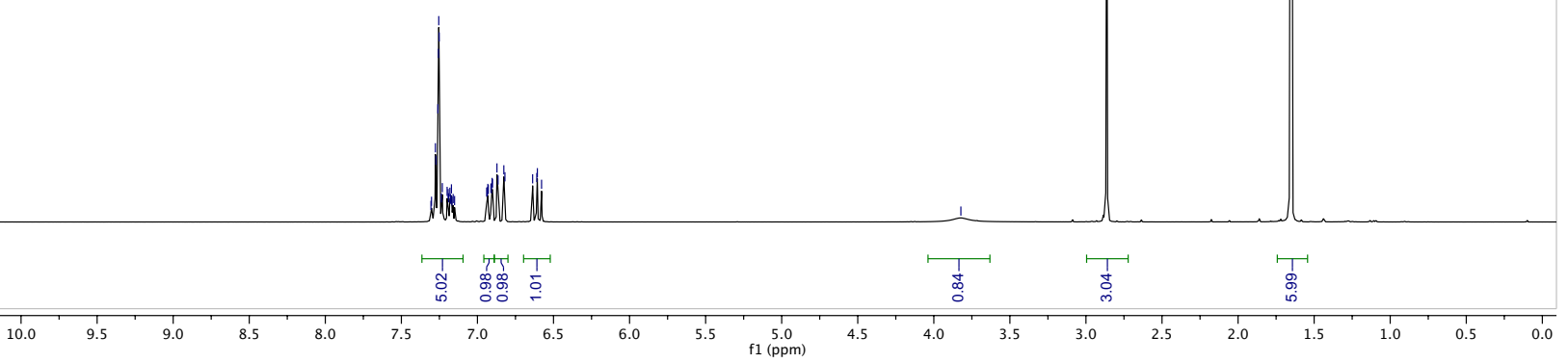

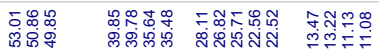

YY Y Y Y V

${ }^{13} \mathrm{C}$ NMR (75 $\mathrm{MHz}, \mathrm{CDCl}_{3}$ )

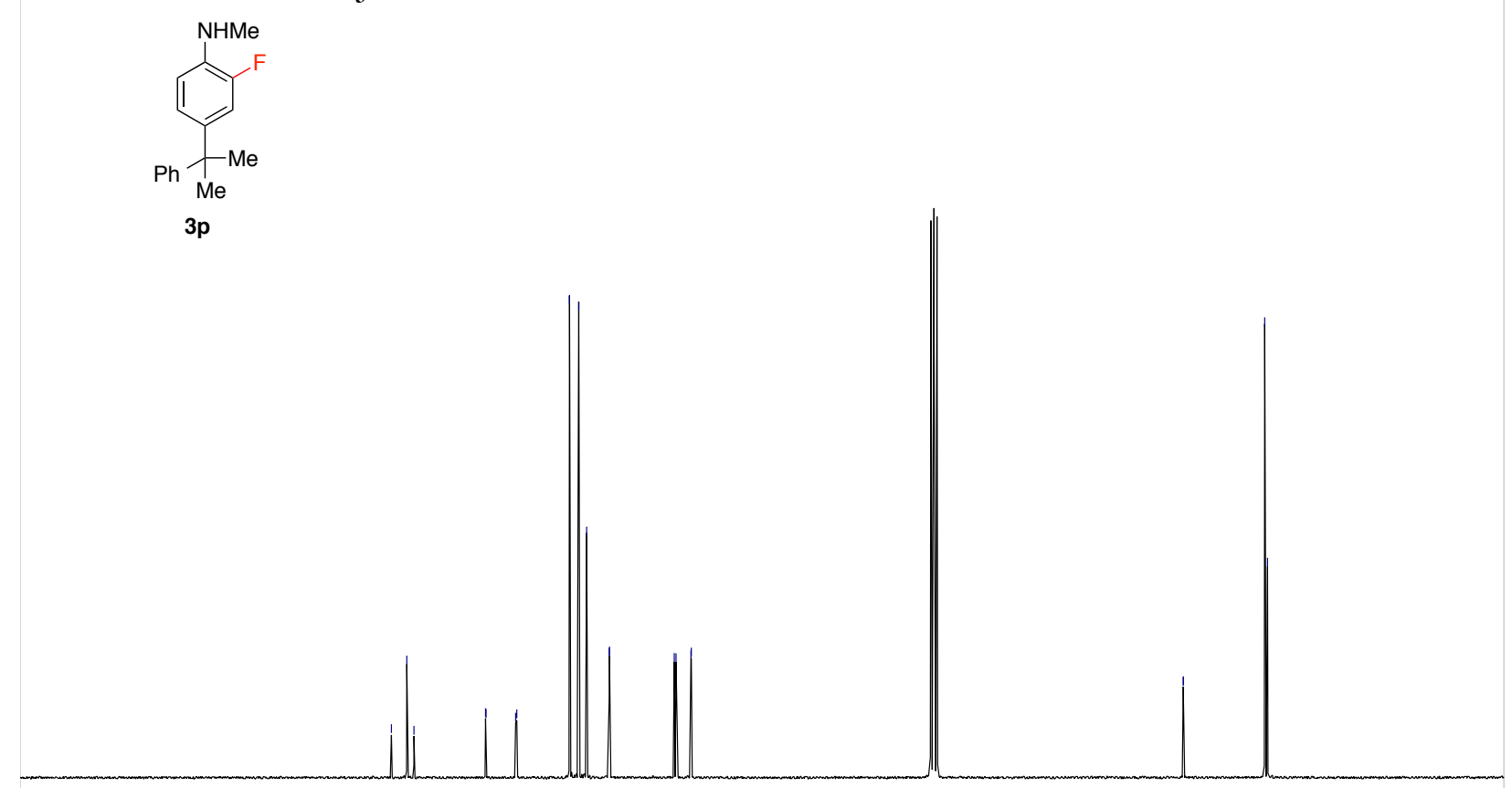

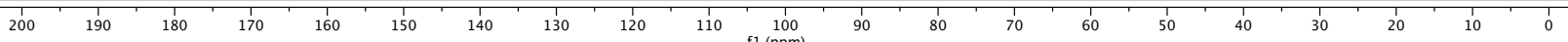


M.
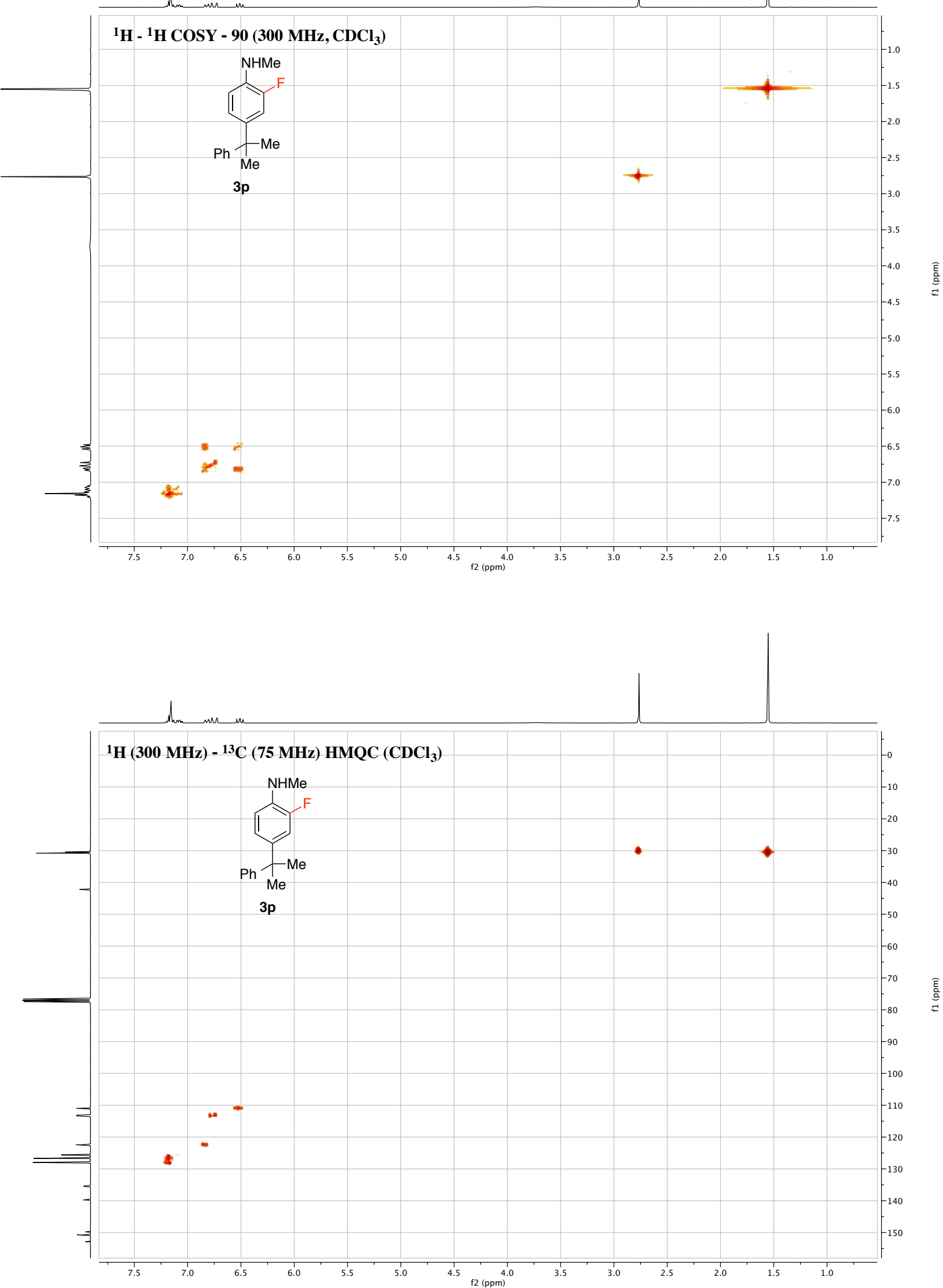


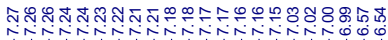
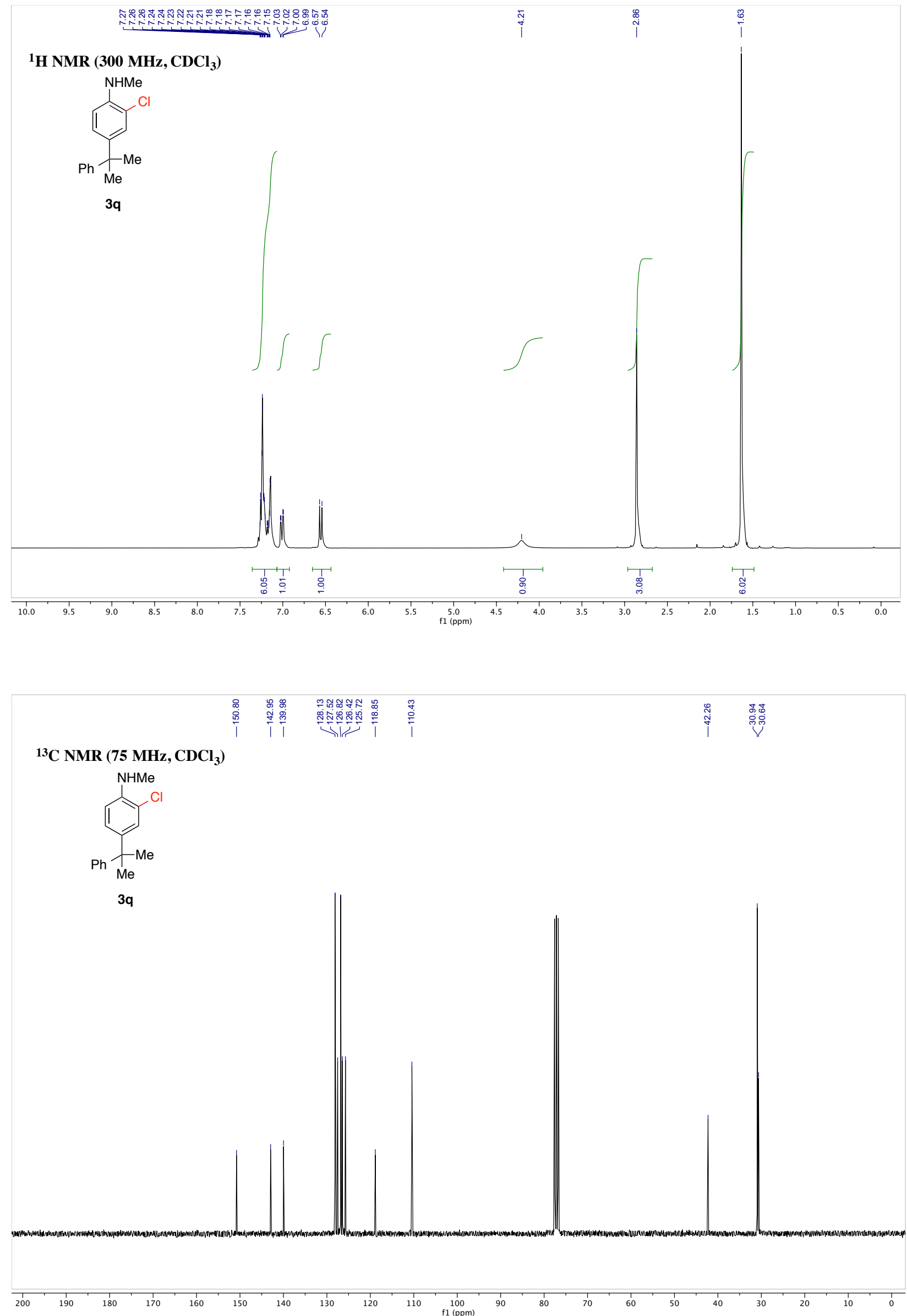


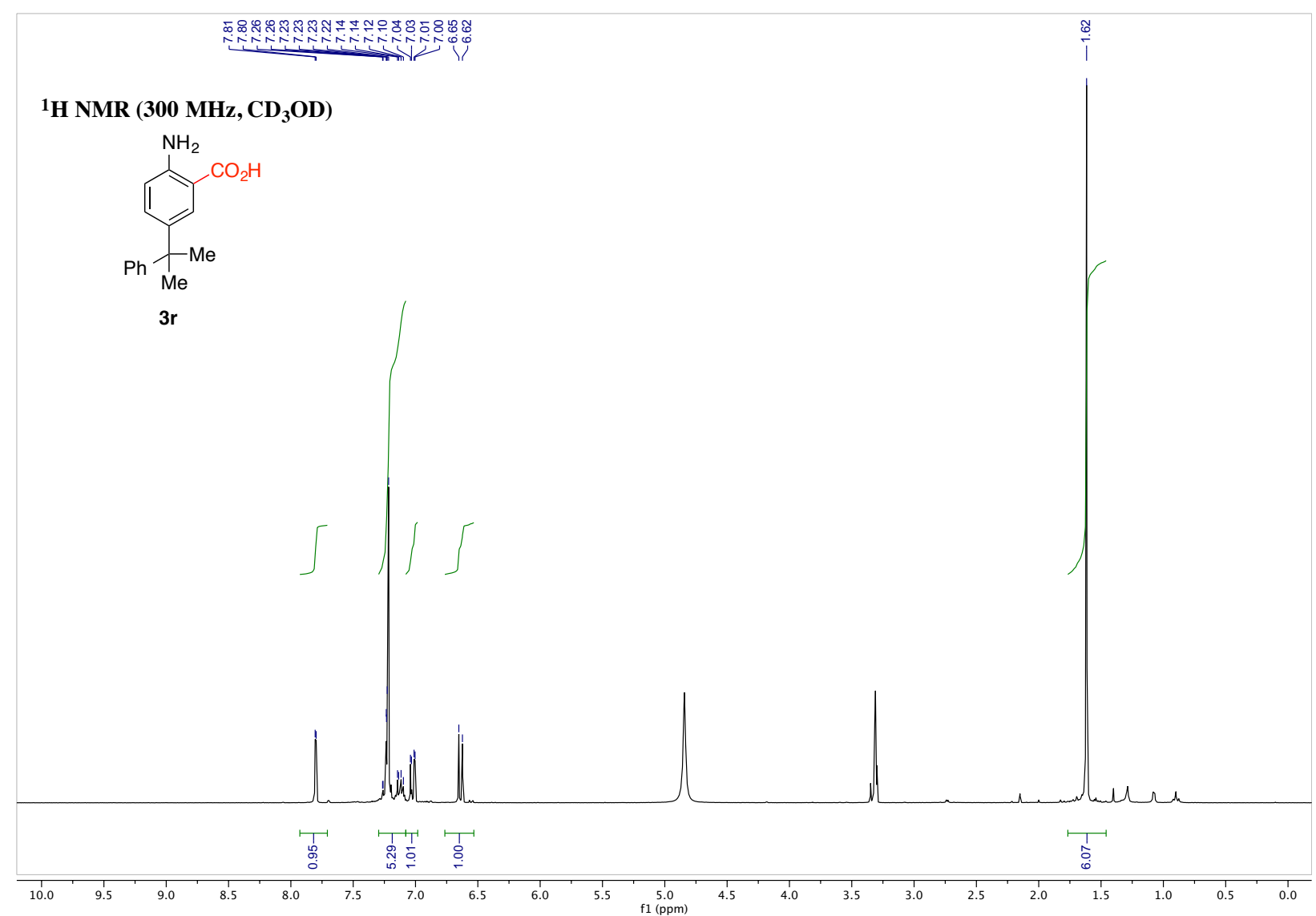

l li

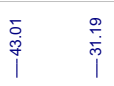

${ }^{13} \mathrm{C}$ NMR (75 MHz, $\left.\mathrm{CD}_{3} \mathrm{OD}\right)$

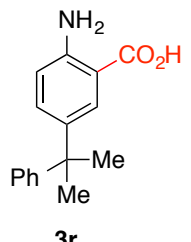

$3 r$

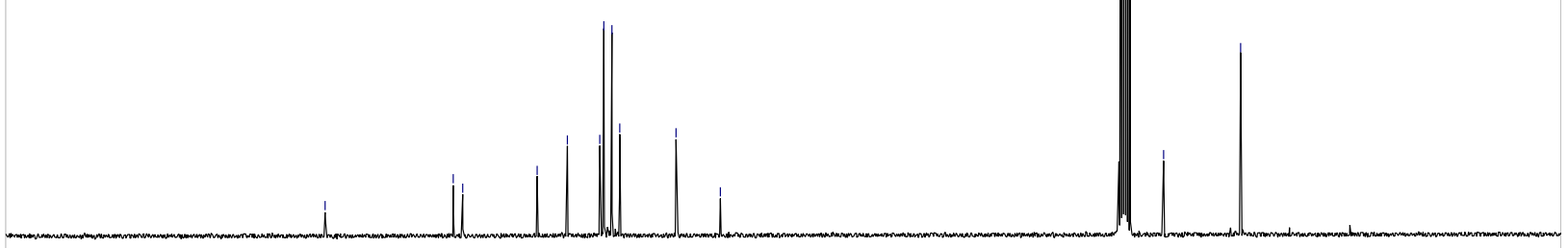

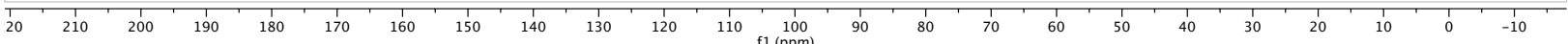



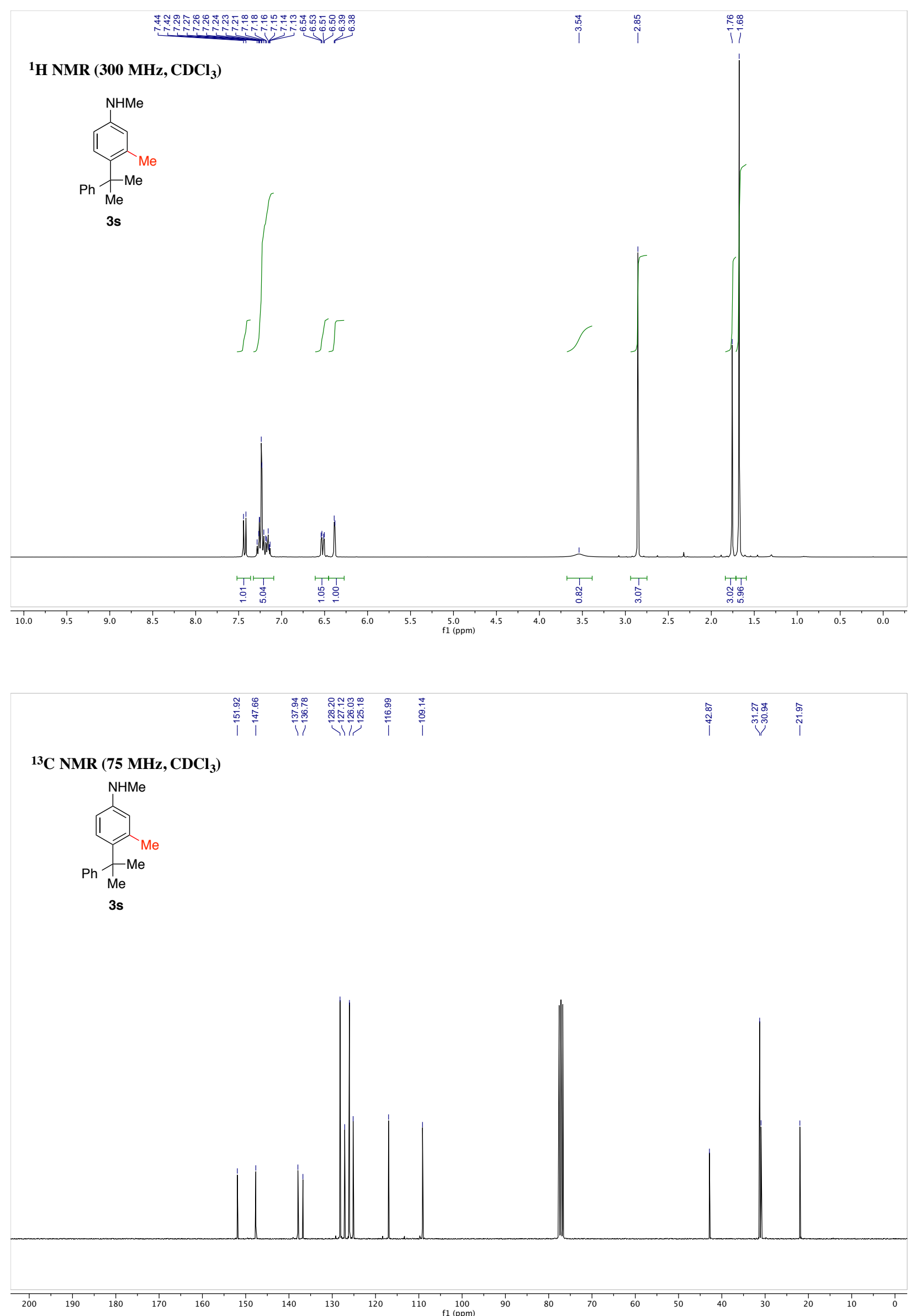

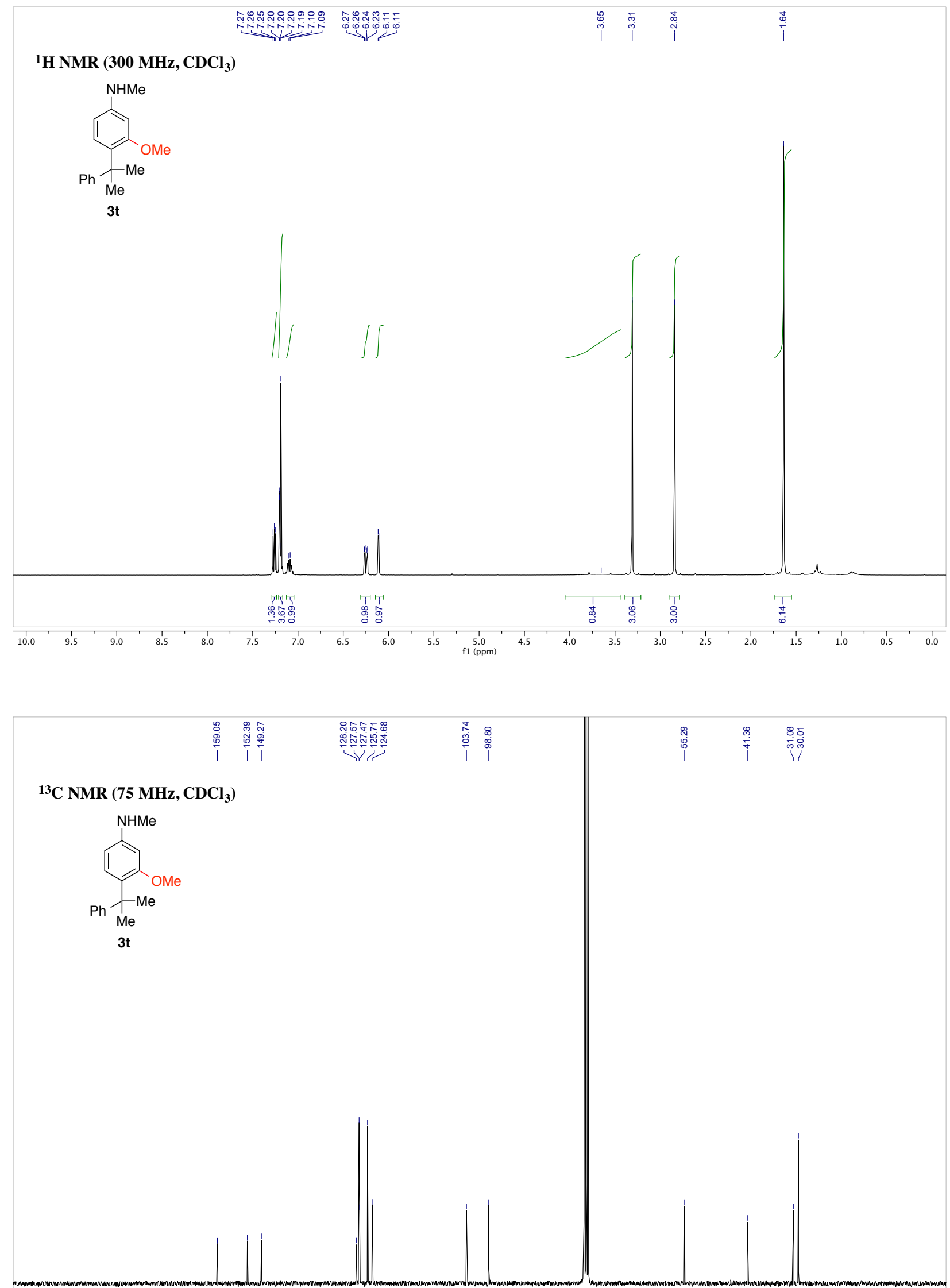

200
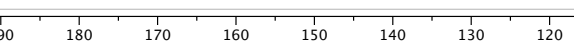

$\begin{array}{cc}110 & 100 \\ & \mathrm{f} 1(\mathrm{ppm})\end{array}$ 

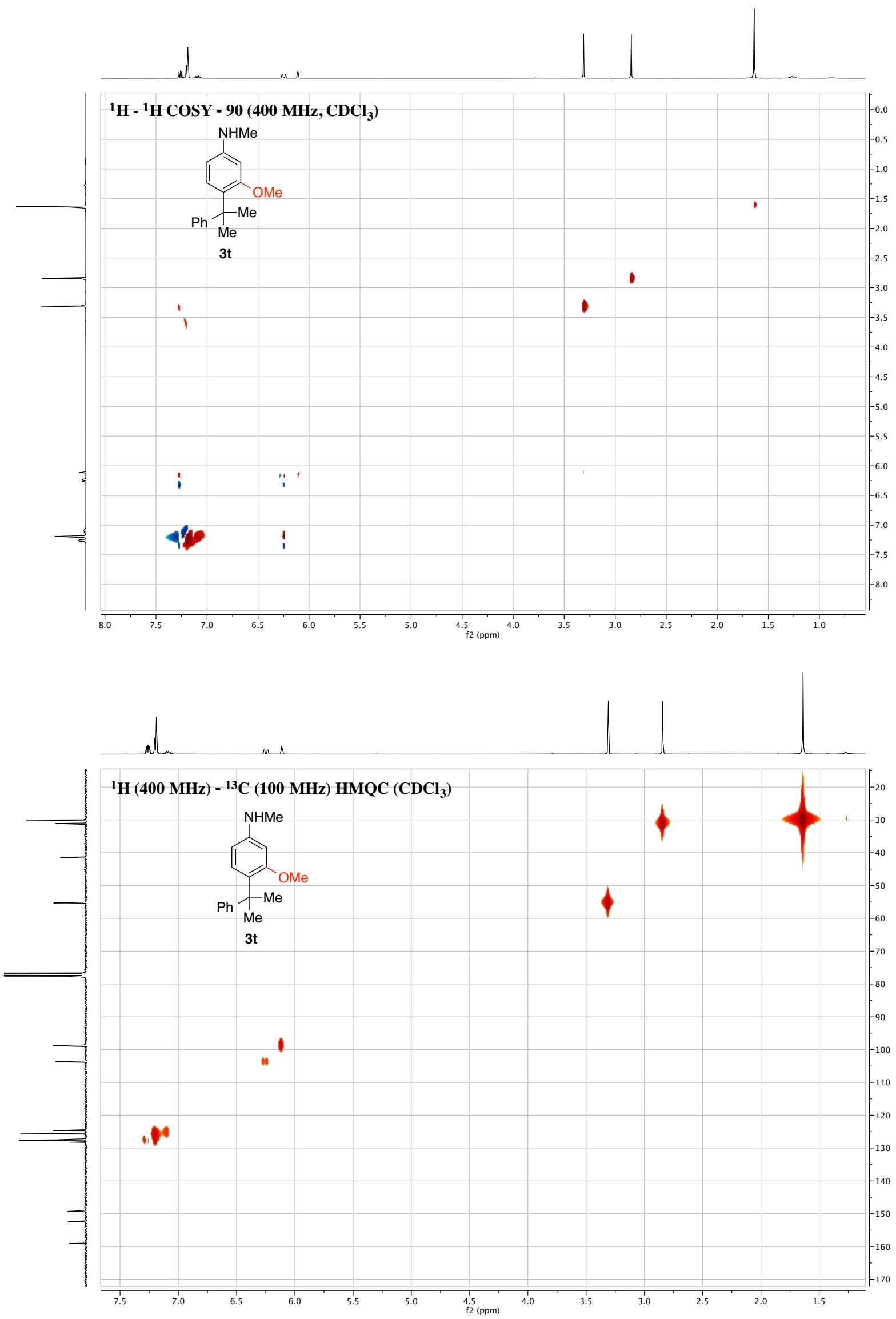
S63
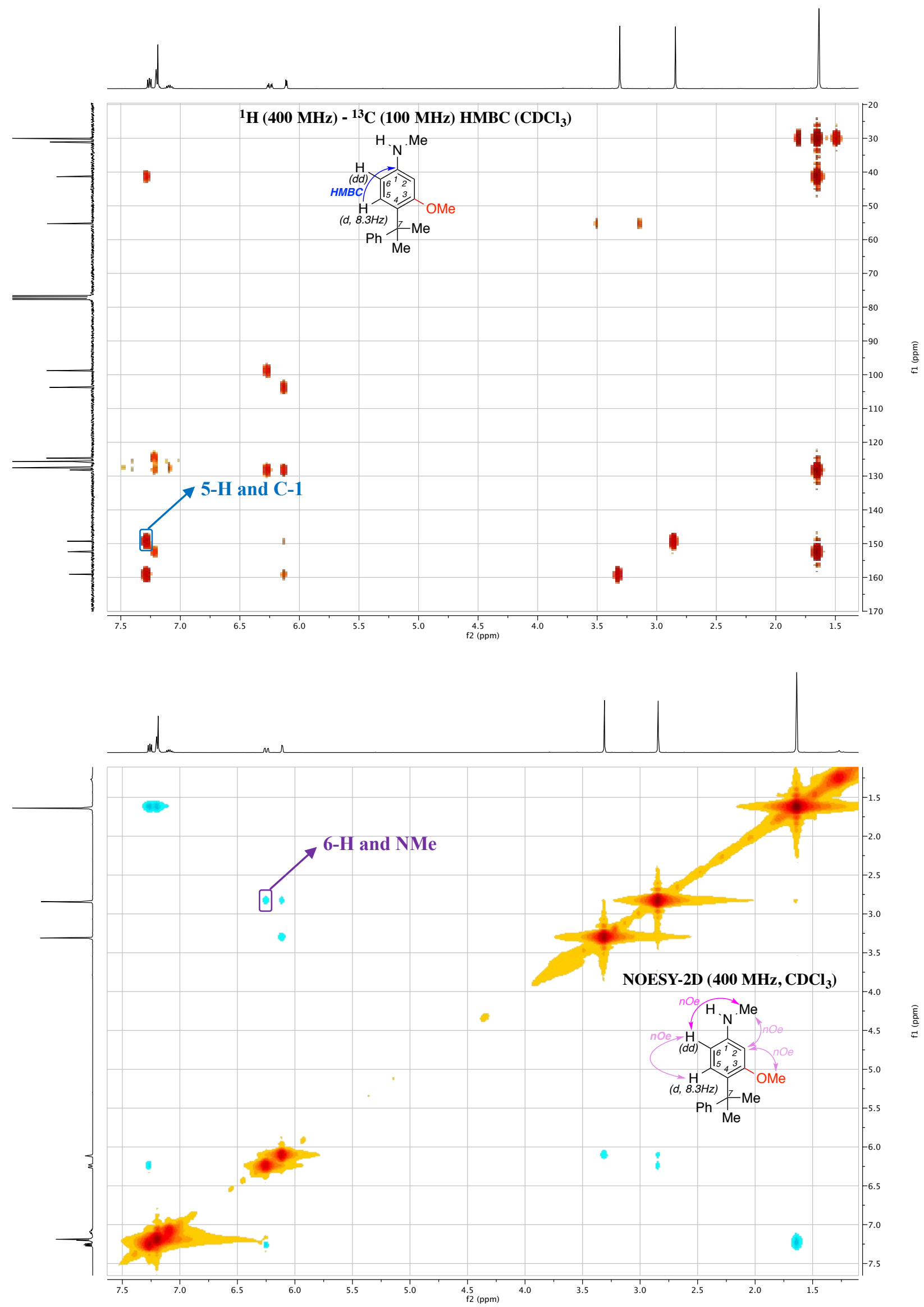

S63 

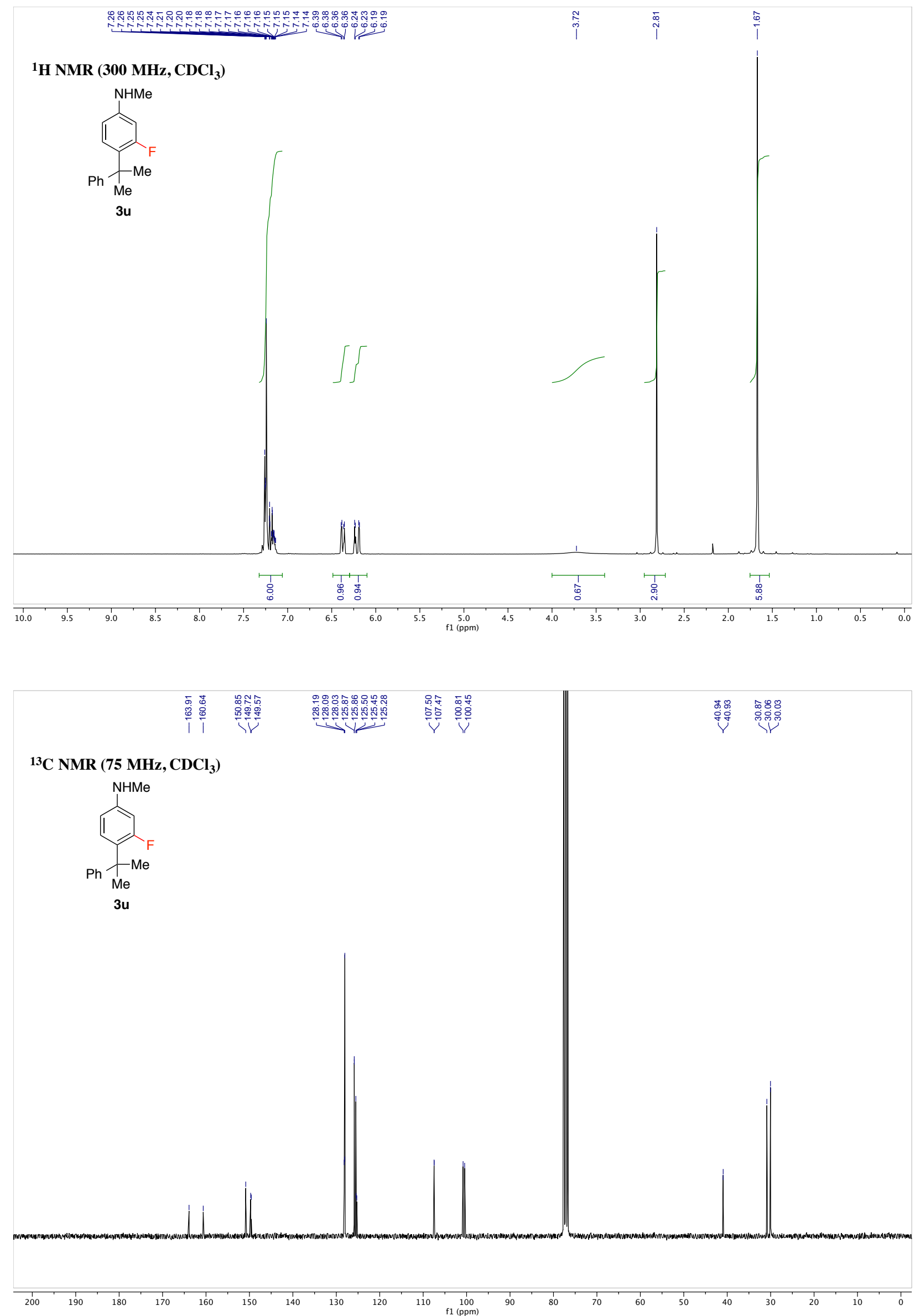
S65
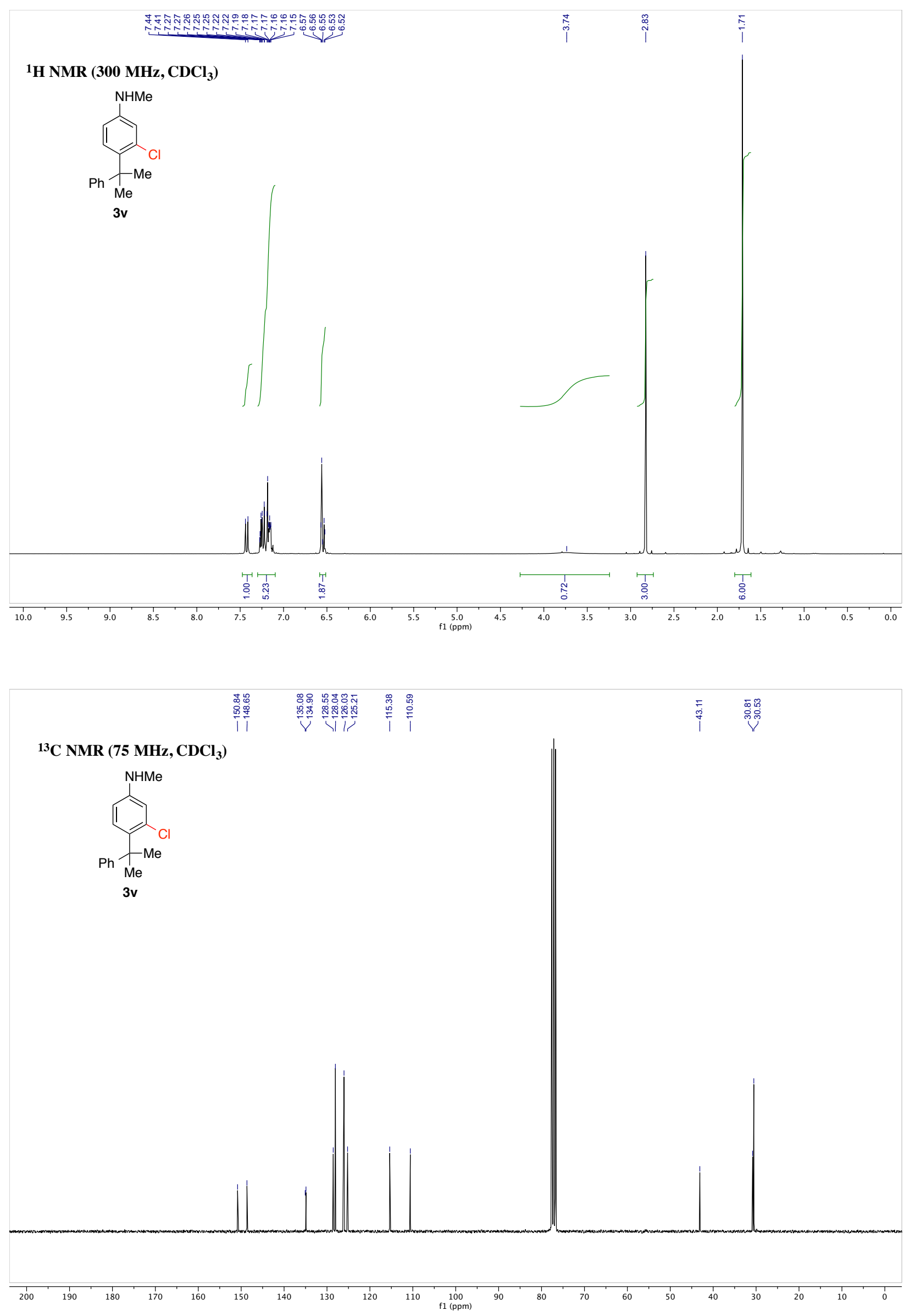

S65 


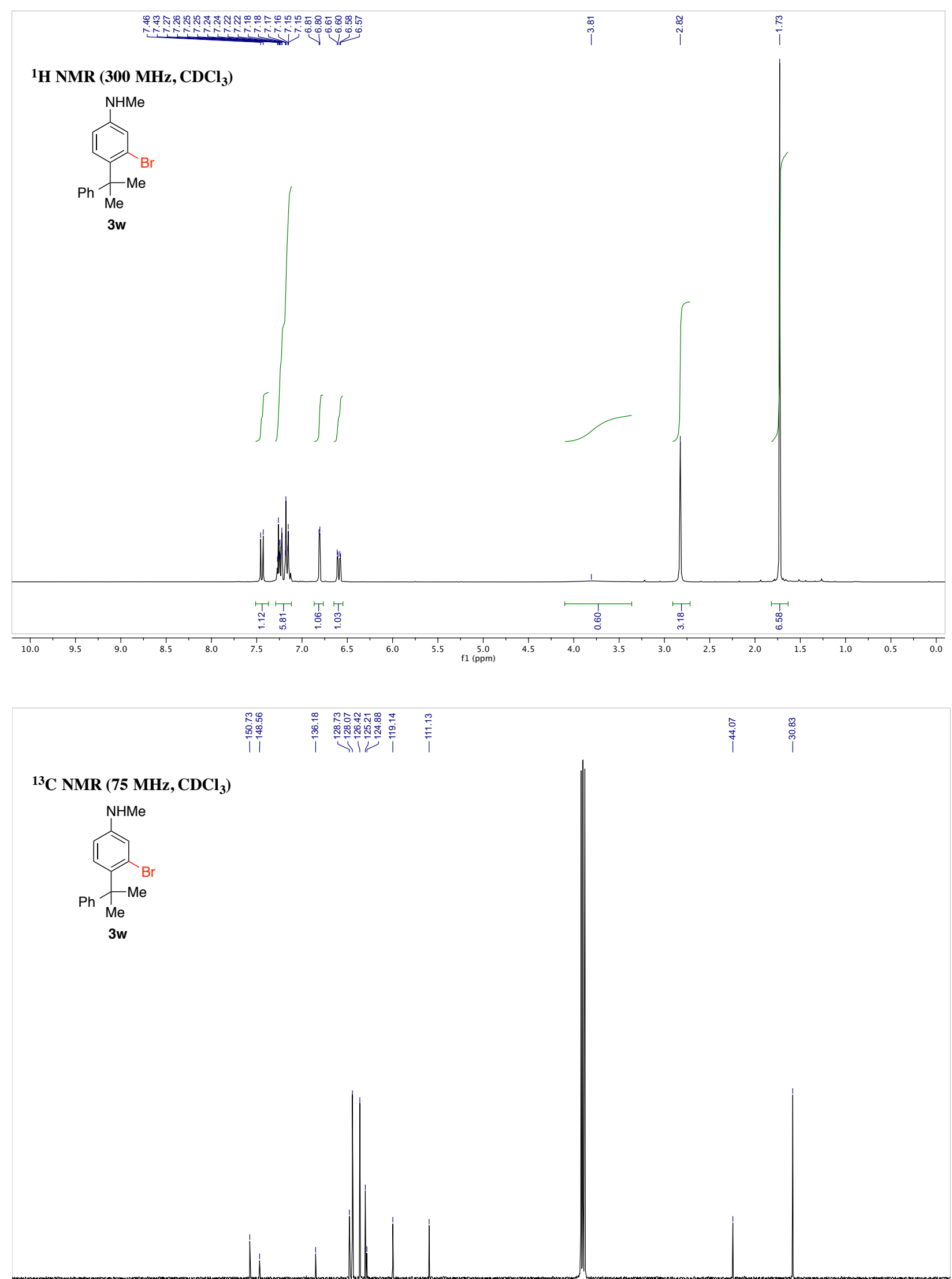

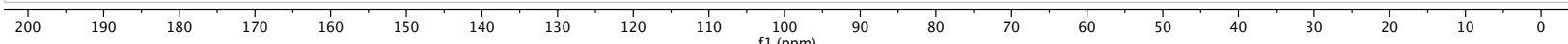


In whe 1

${ }^{1} \mathrm{H}$ - ${ }^{1} \mathrm{H} \mathrm{COSY}$ - $90\left(300 \mathrm{MHz}, \mathrm{CDCl}_{3}\right)$

NHMe

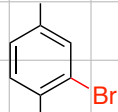

$\mathrm{Ph}$

Me

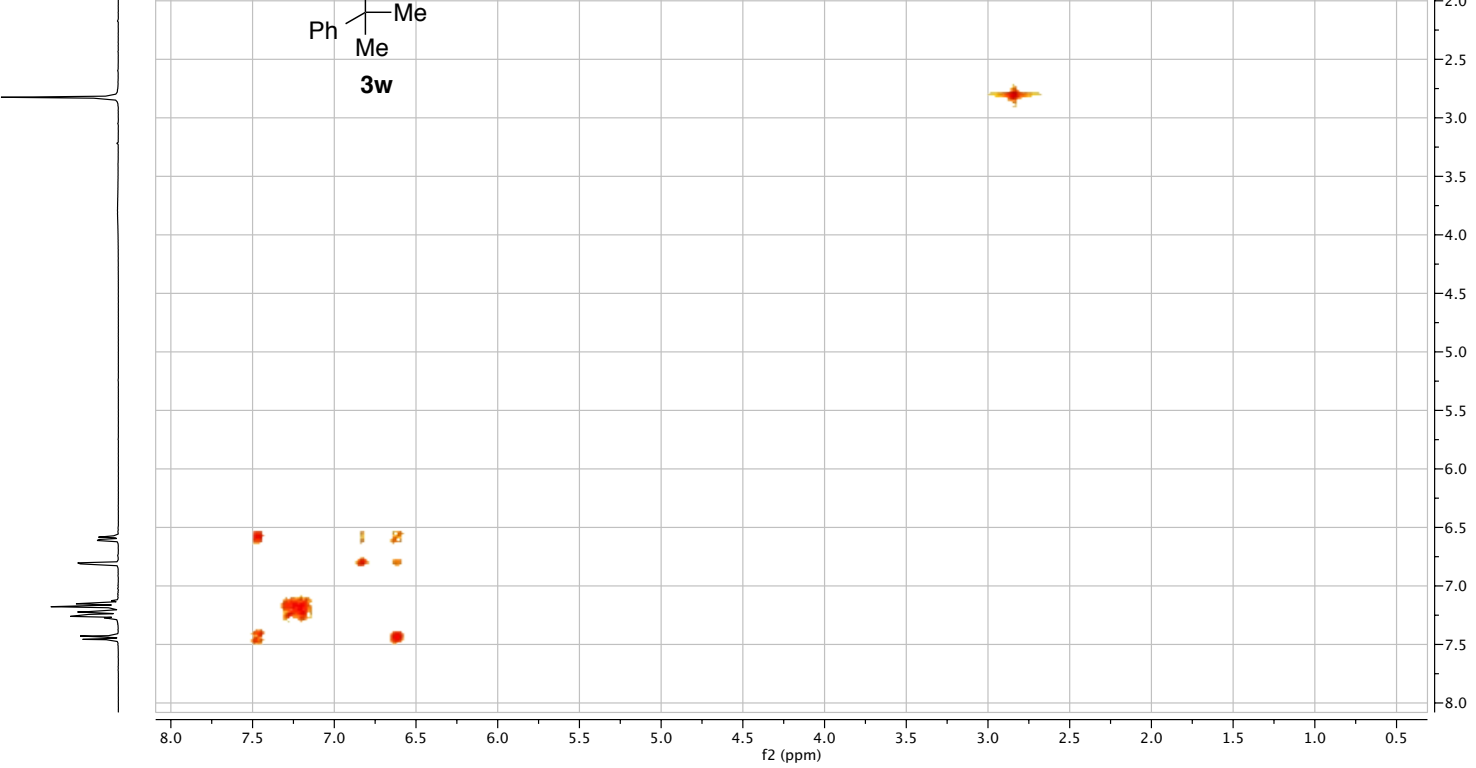

Inll L

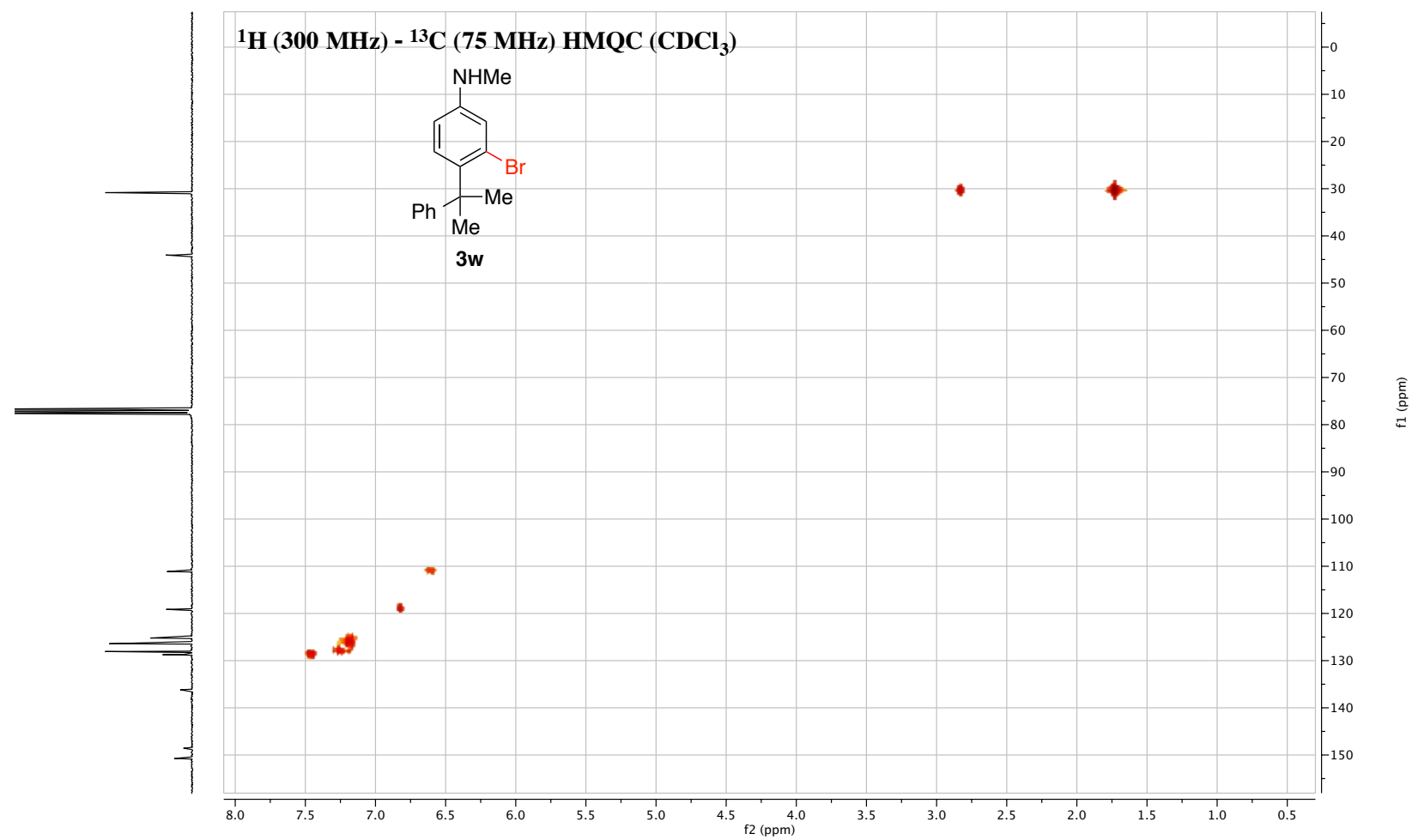



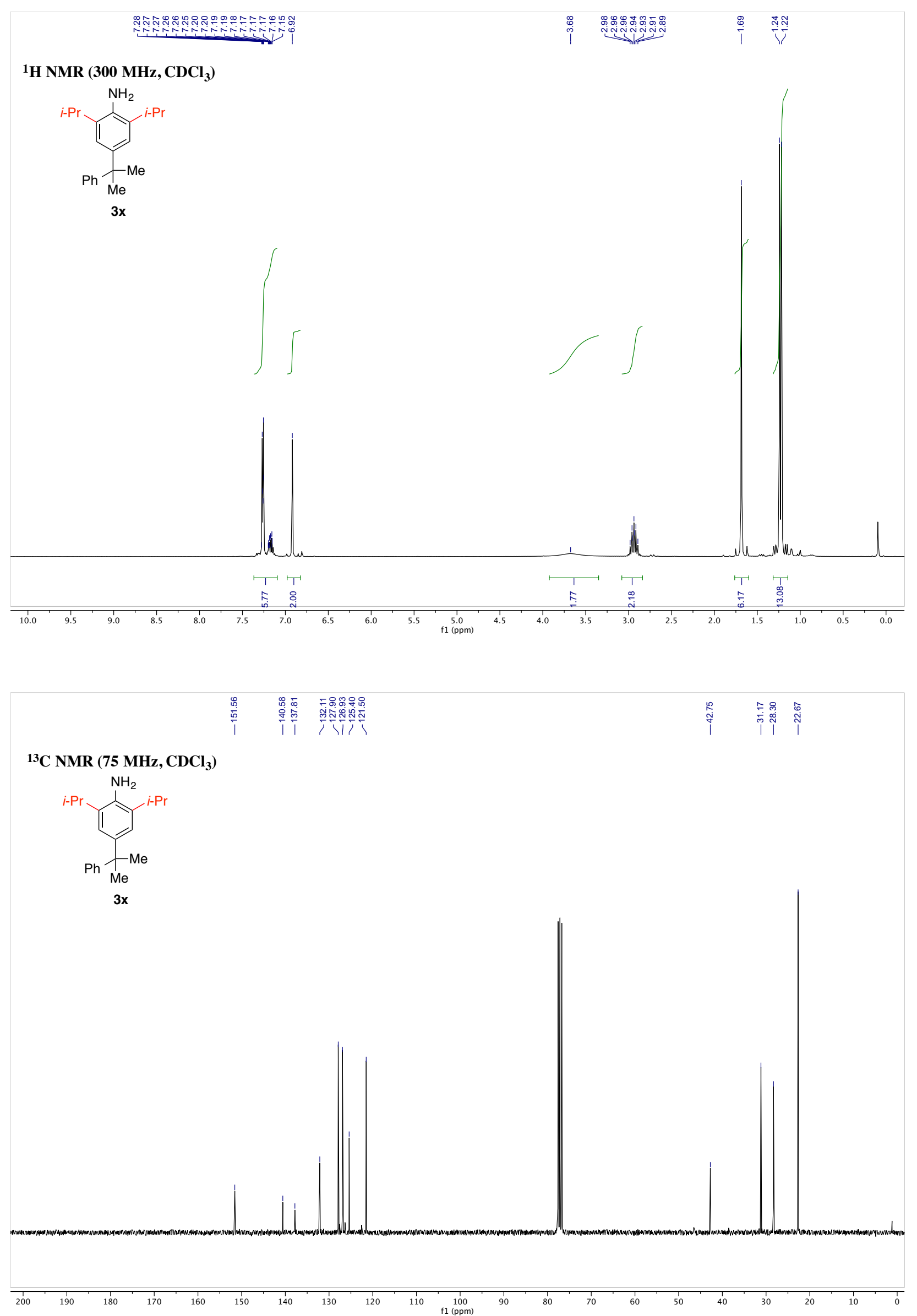


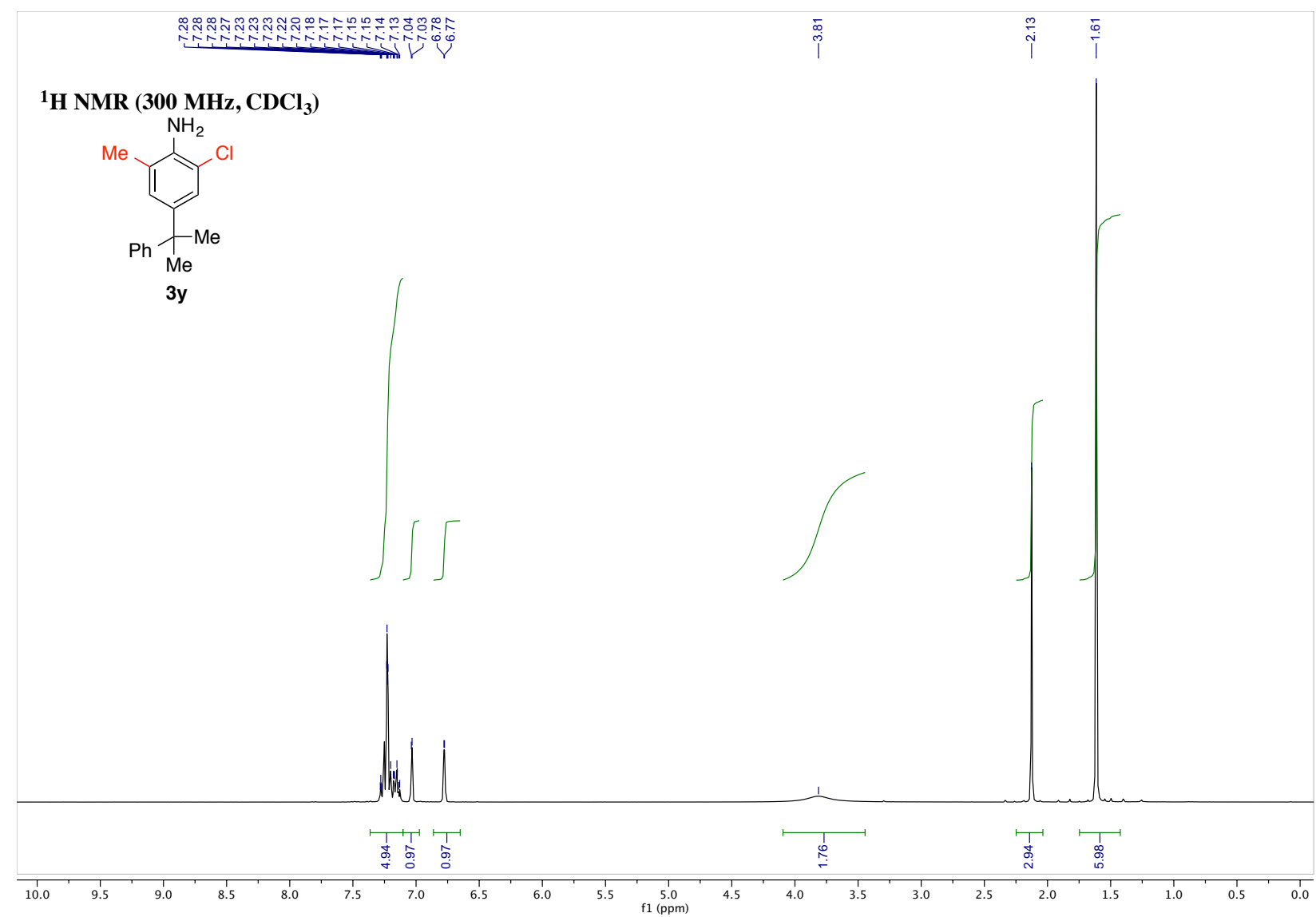

\begin{tabular}{|c|c|c|}
\hline 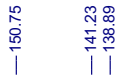 & 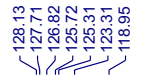 & \\
\hline
\end{tabular}

${ }^{13} \mathrm{C}$ NMR (75 $\left.\mathrm{MHz}, \mathrm{CDCl}_{3}\right)$
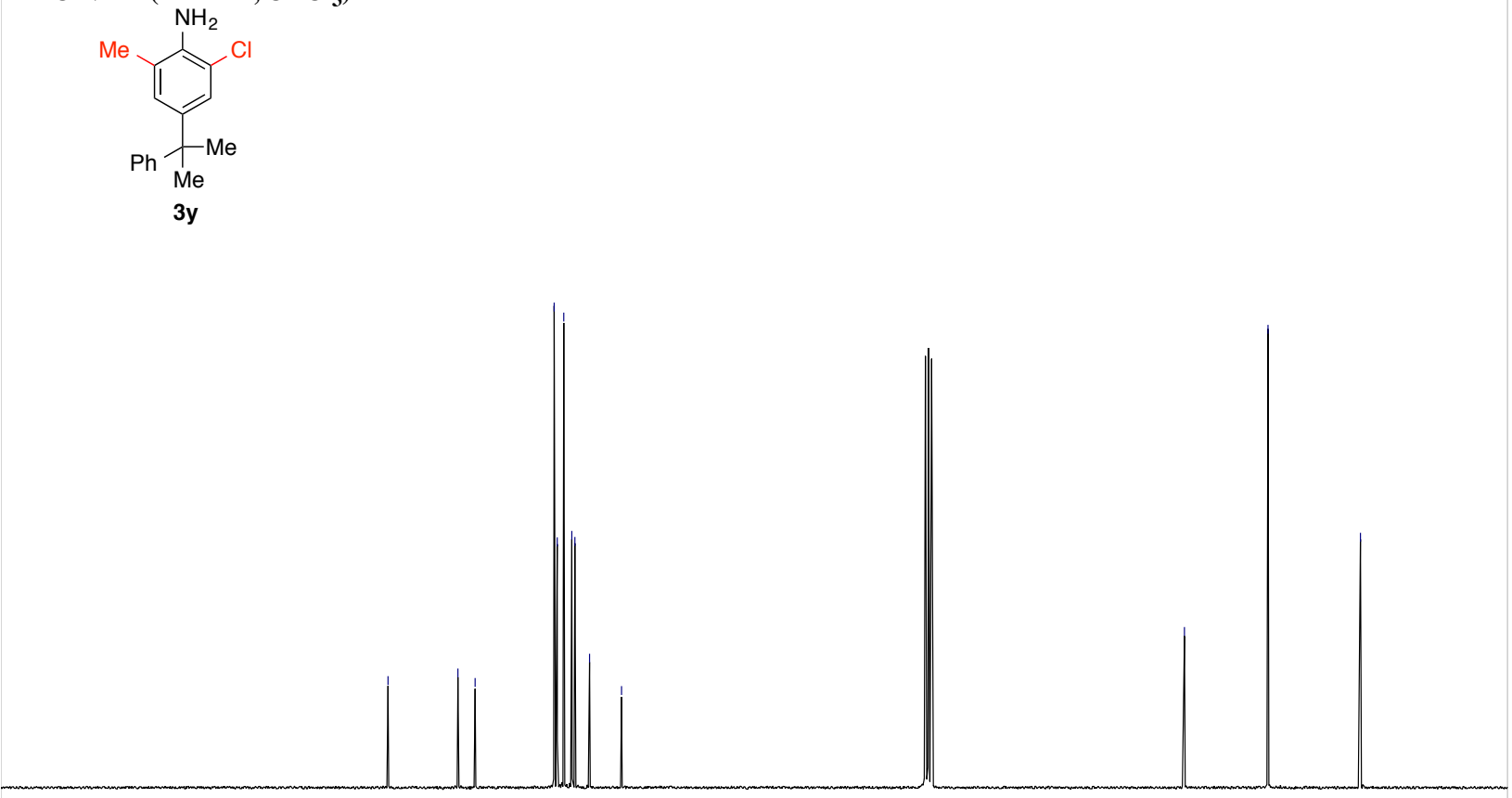

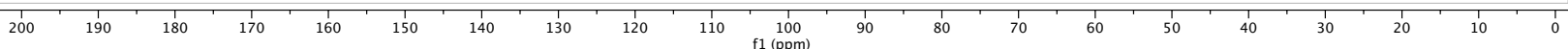



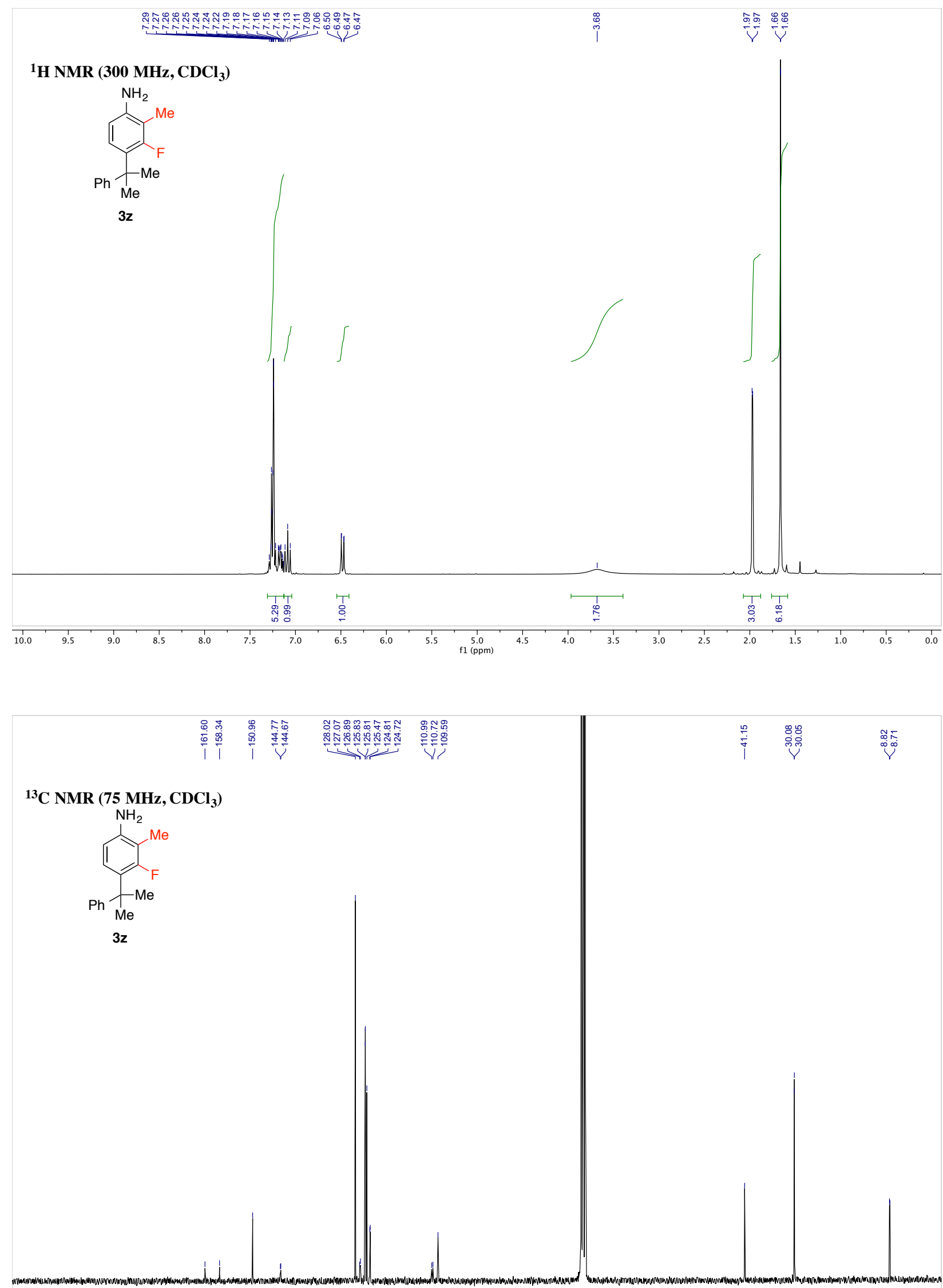

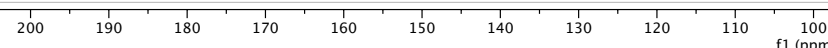



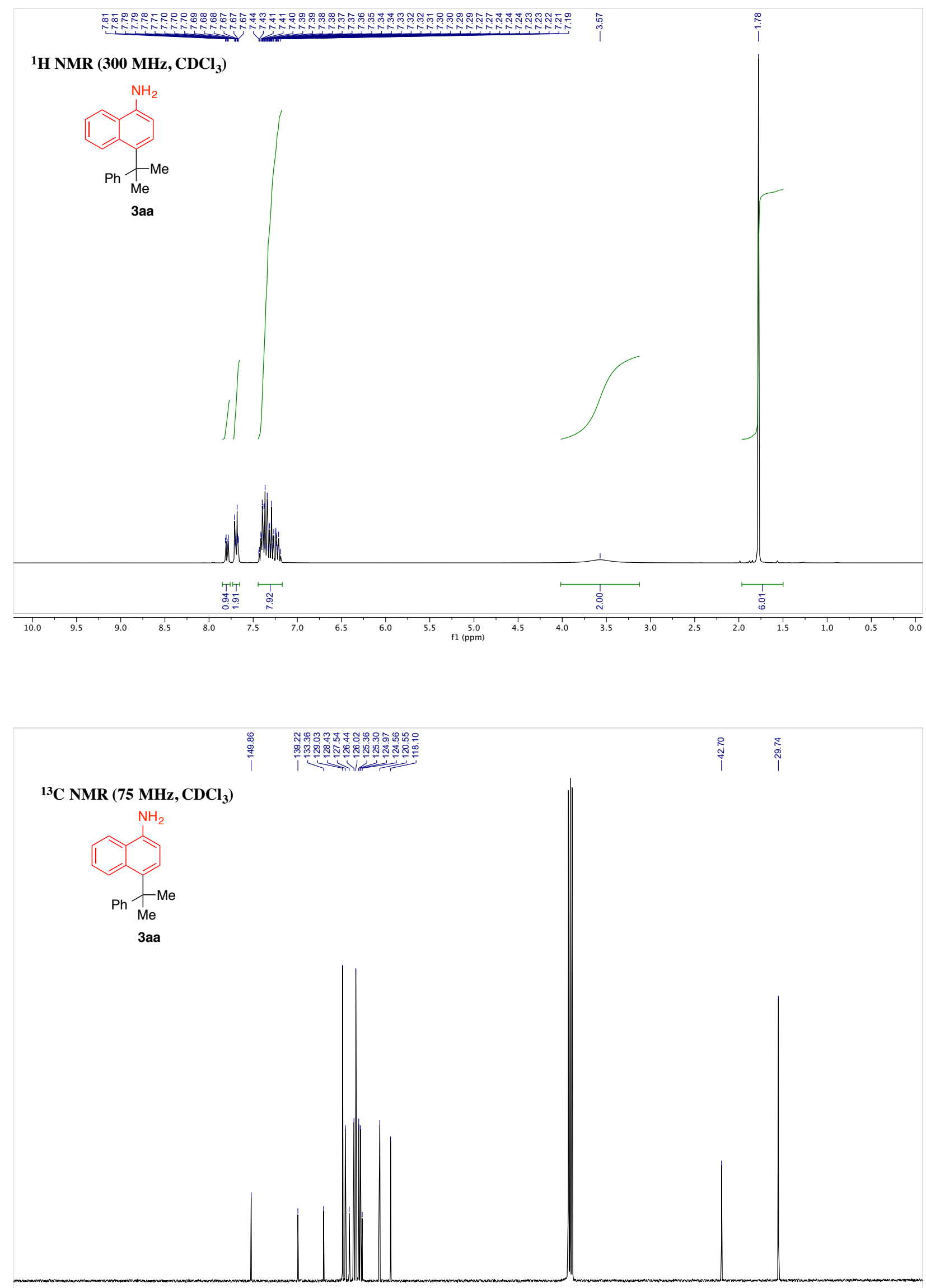

200
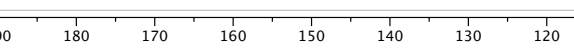


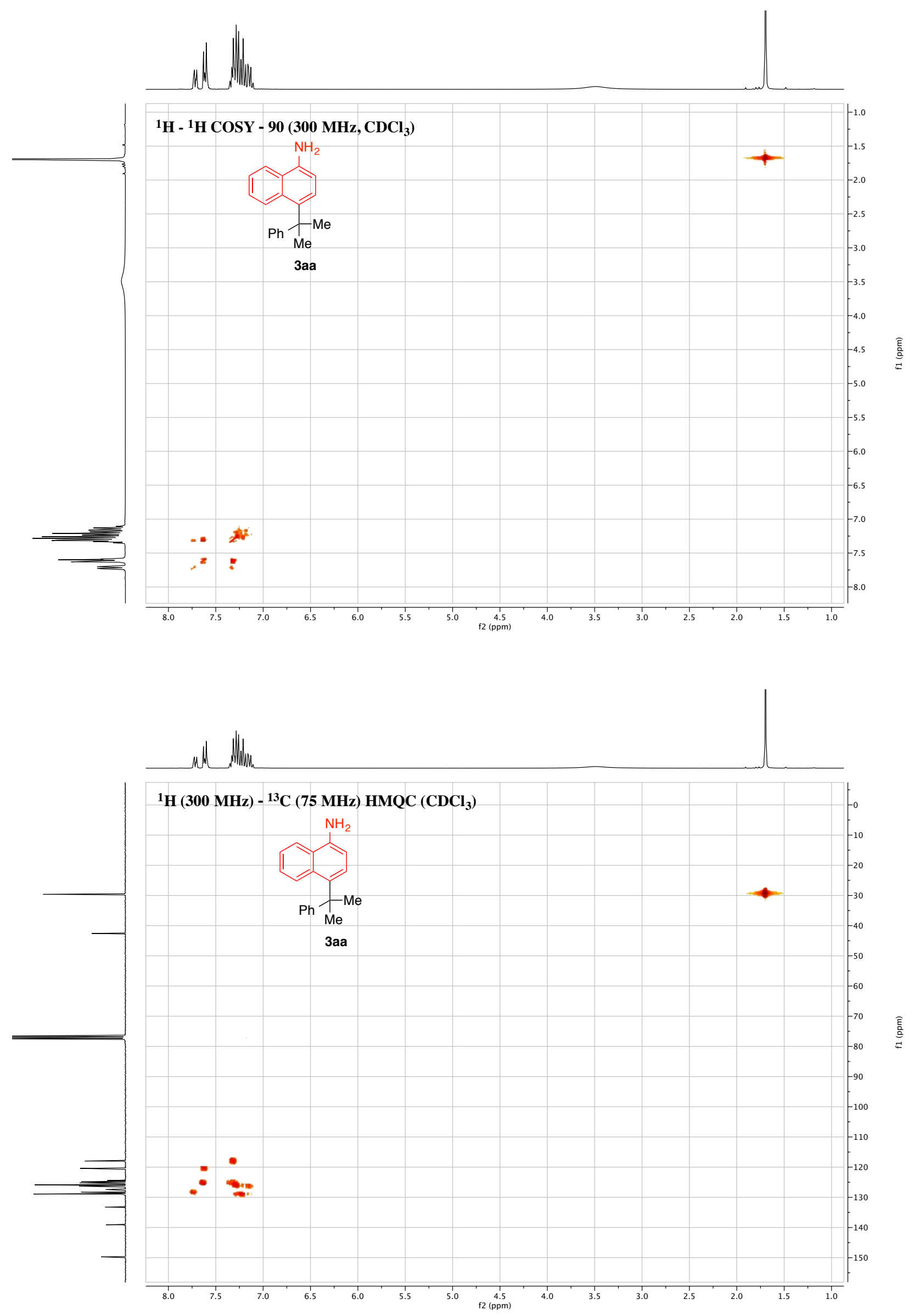




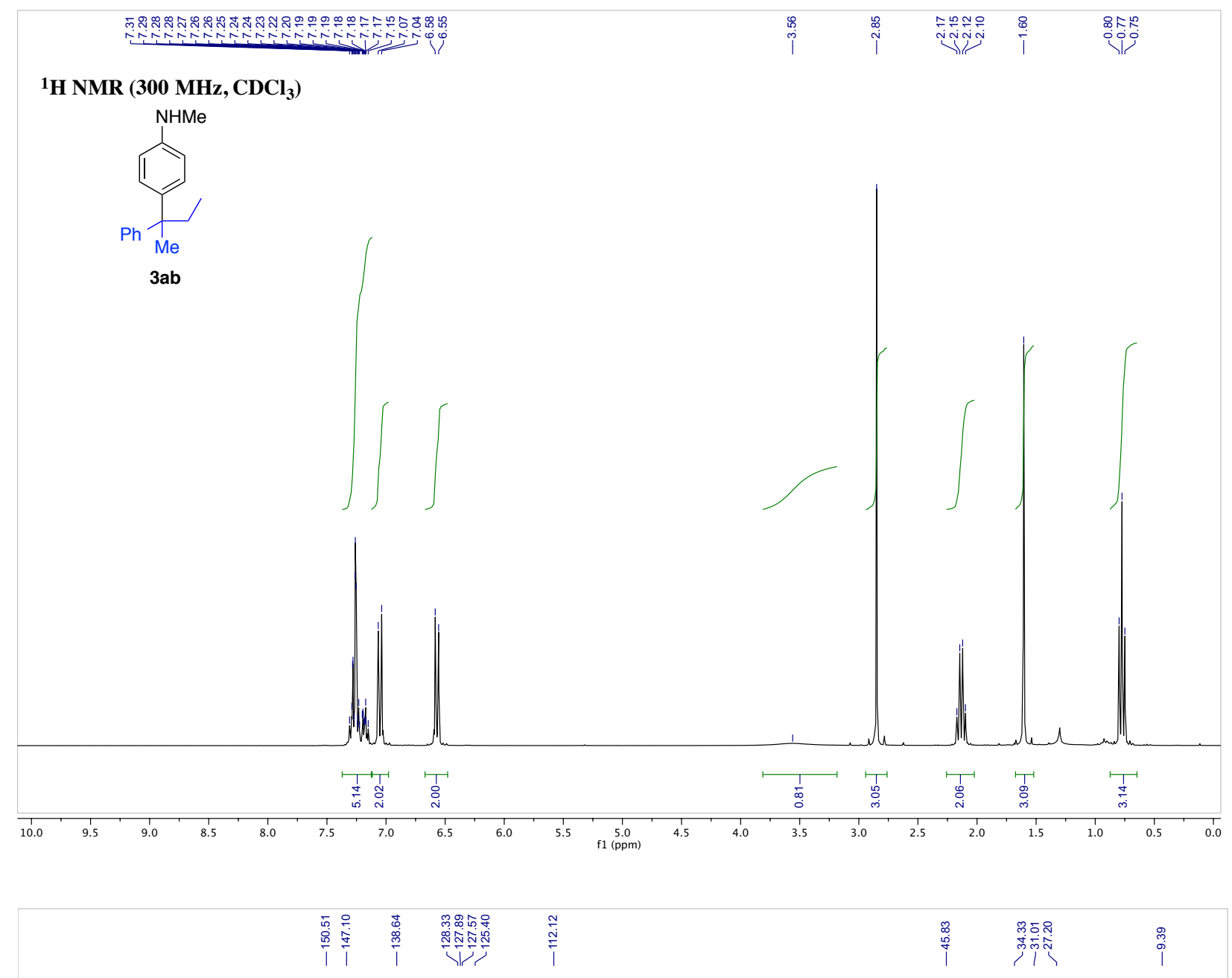

${ }^{13} \mathrm{C}$ NMR (75 MHz, $\left.\mathrm{CDCl}_{3}\right)$
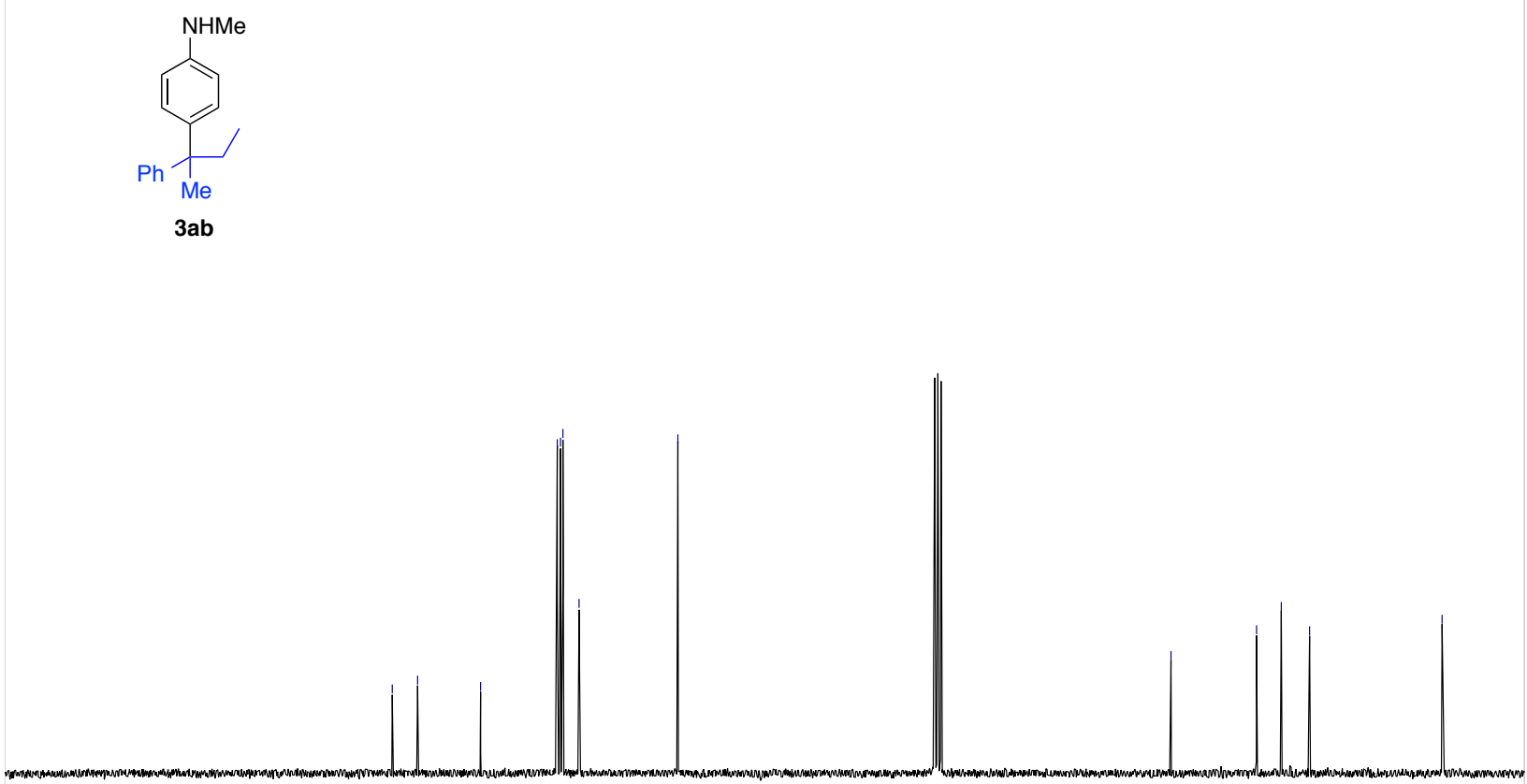
S74
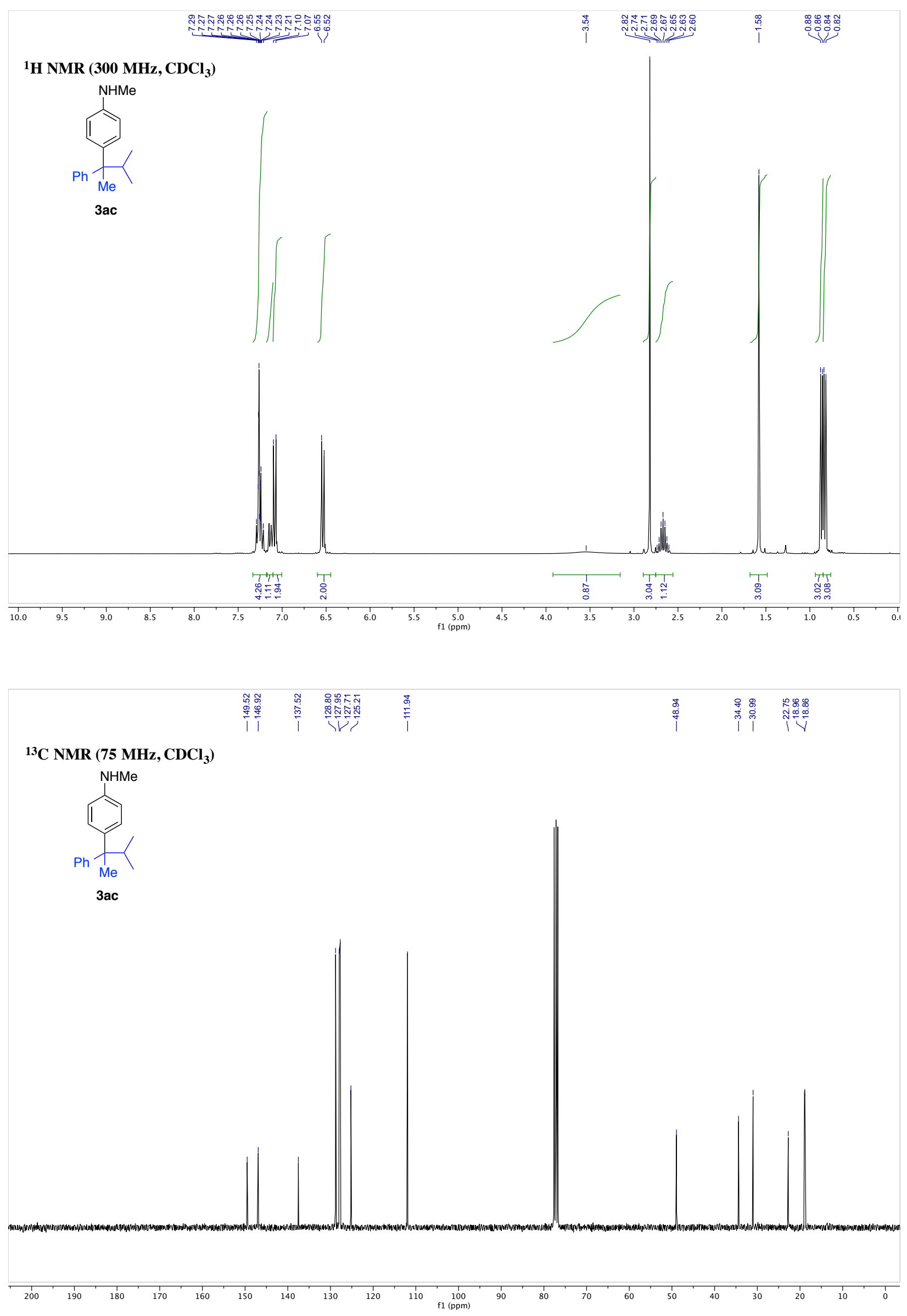

S74 

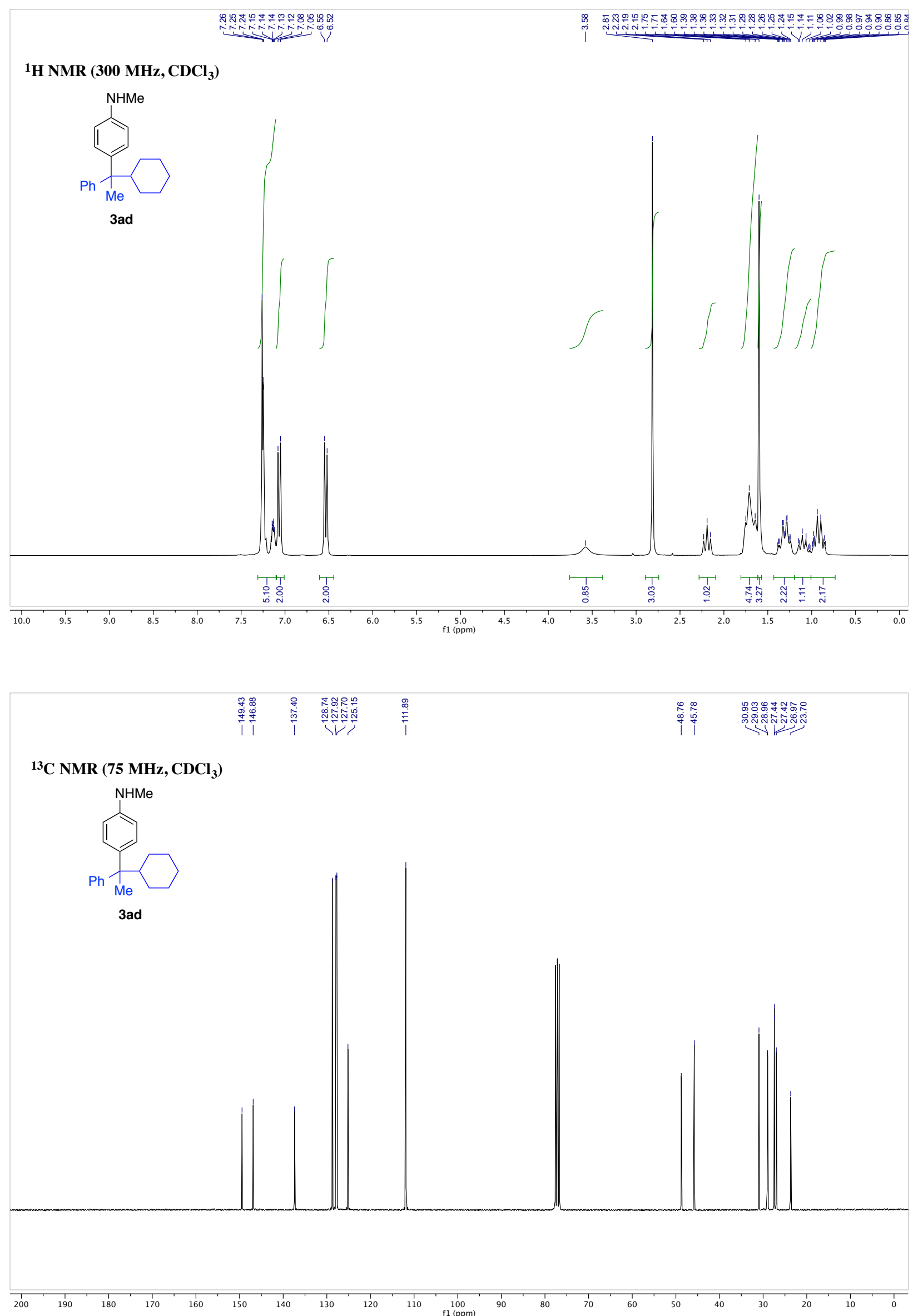


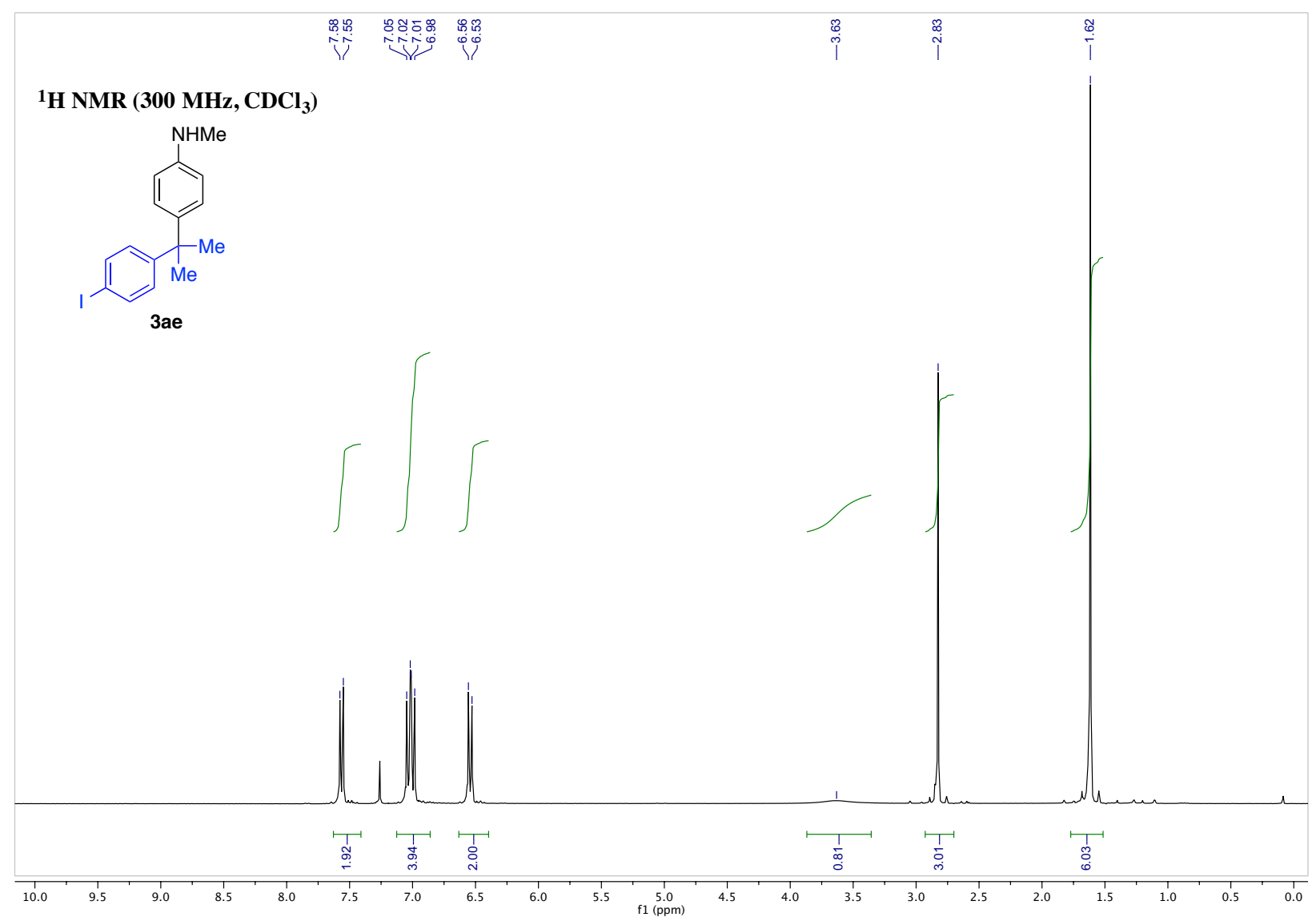

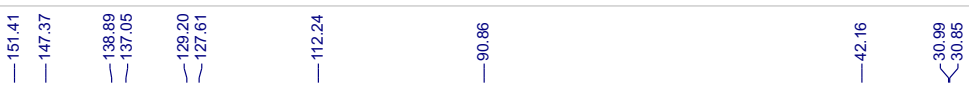

${ }^{13} \mathrm{C}$ NMR (75 $\left.\mathrm{MHz}, \mathrm{CDCl}_{3}\right)$

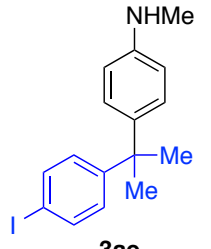

3ae

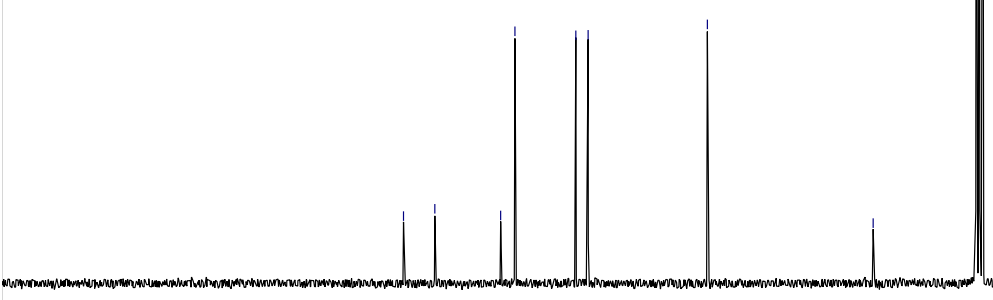



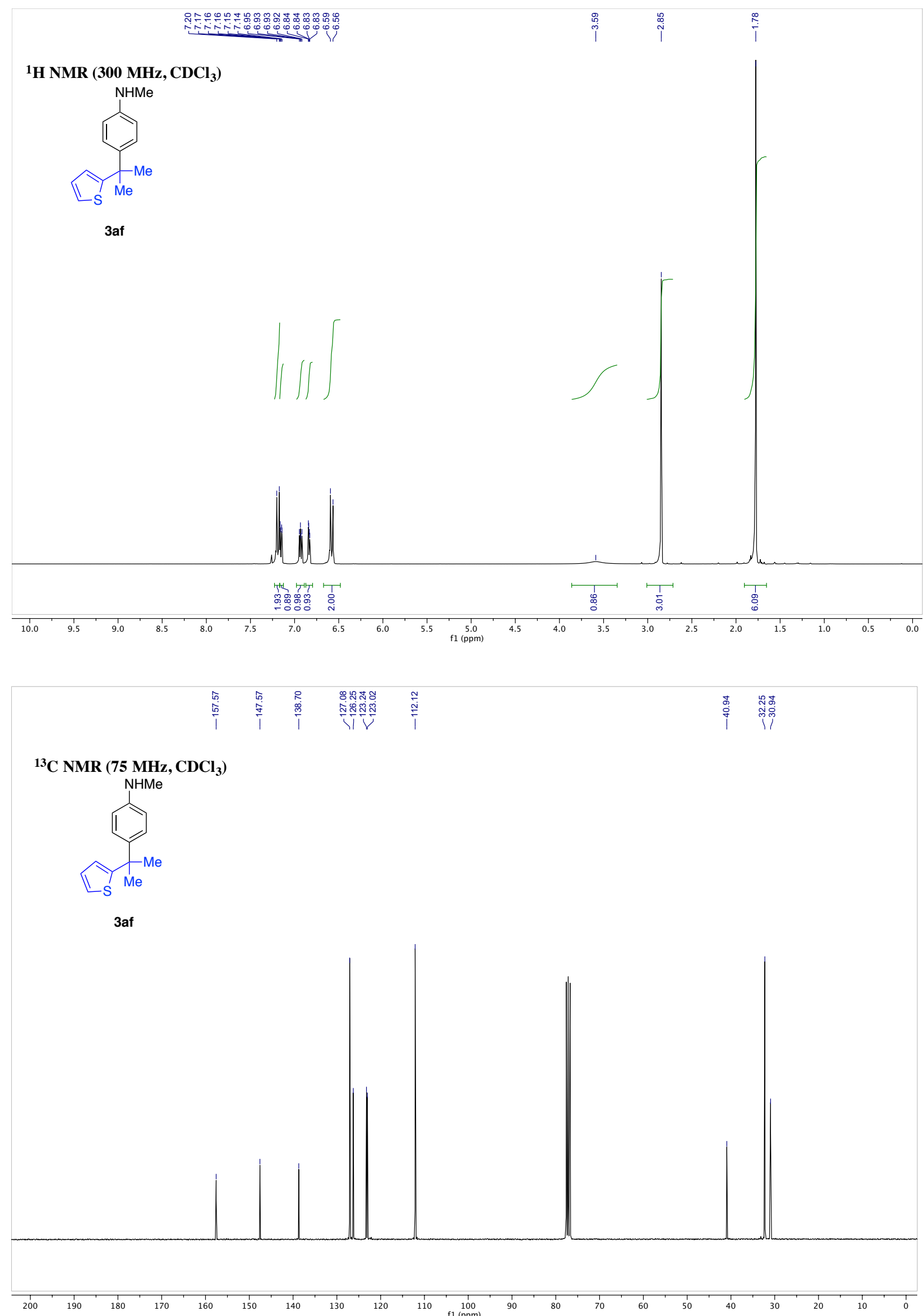
S78
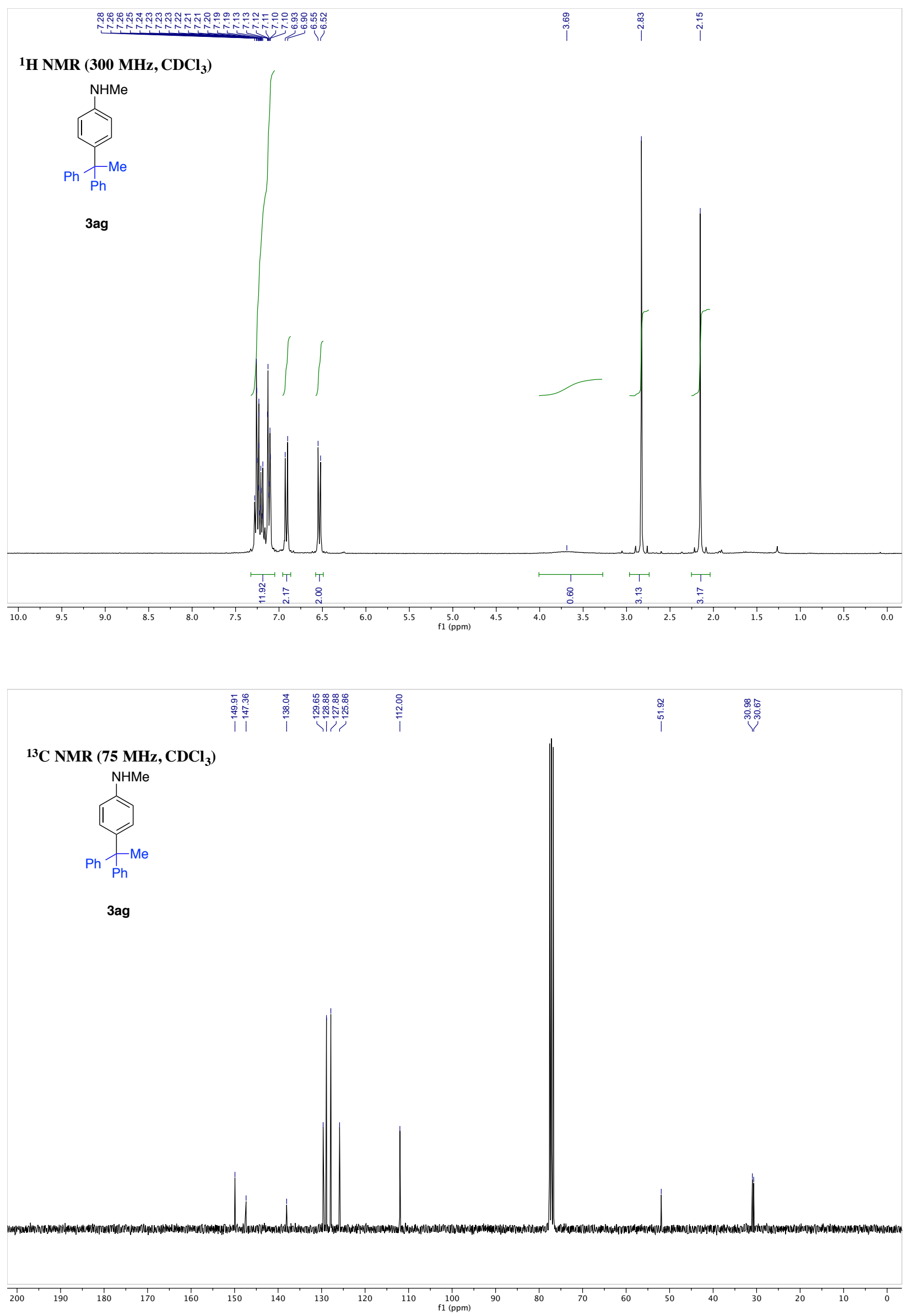

S78 


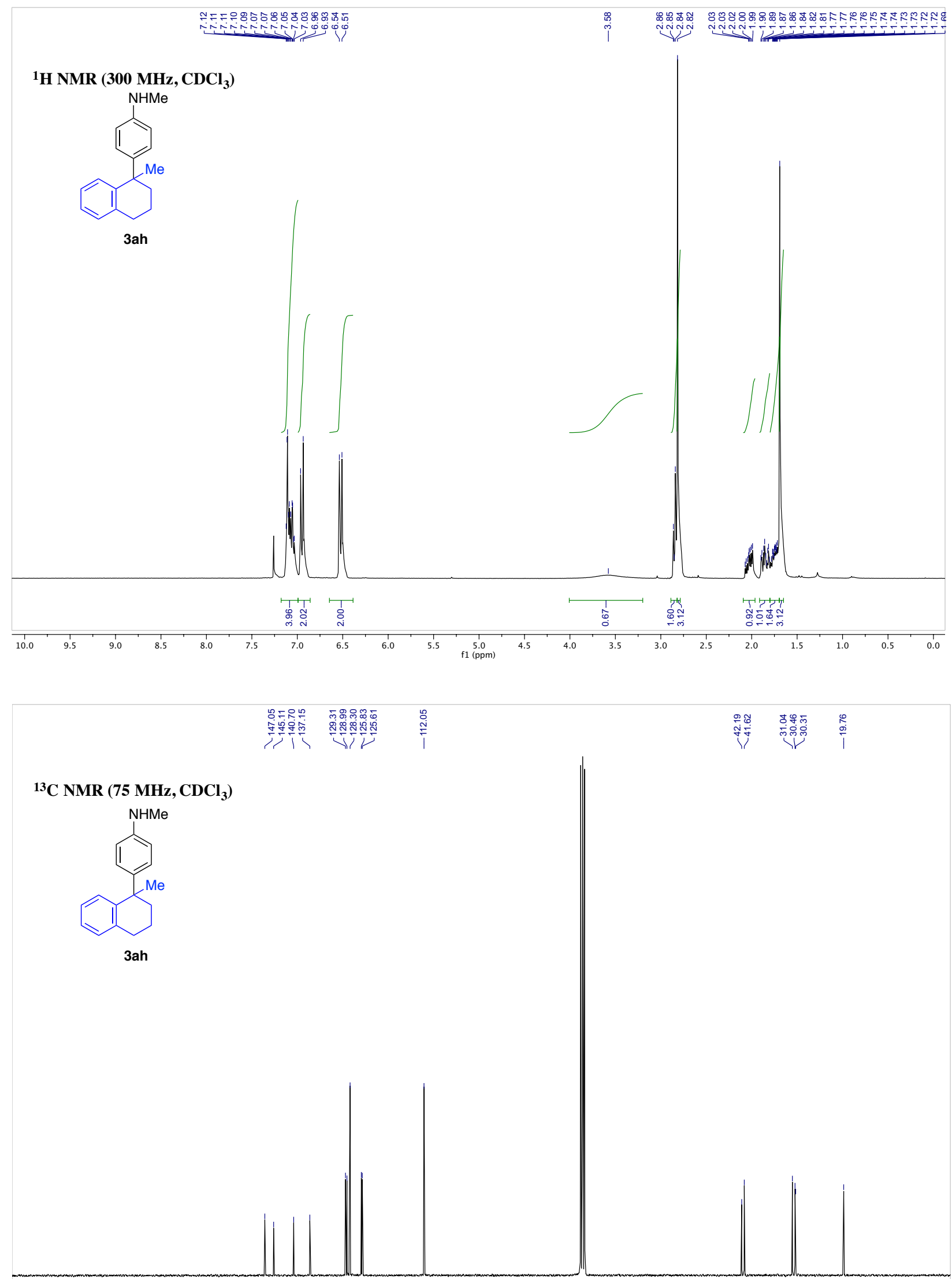

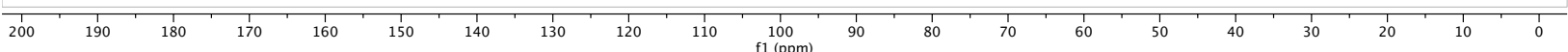




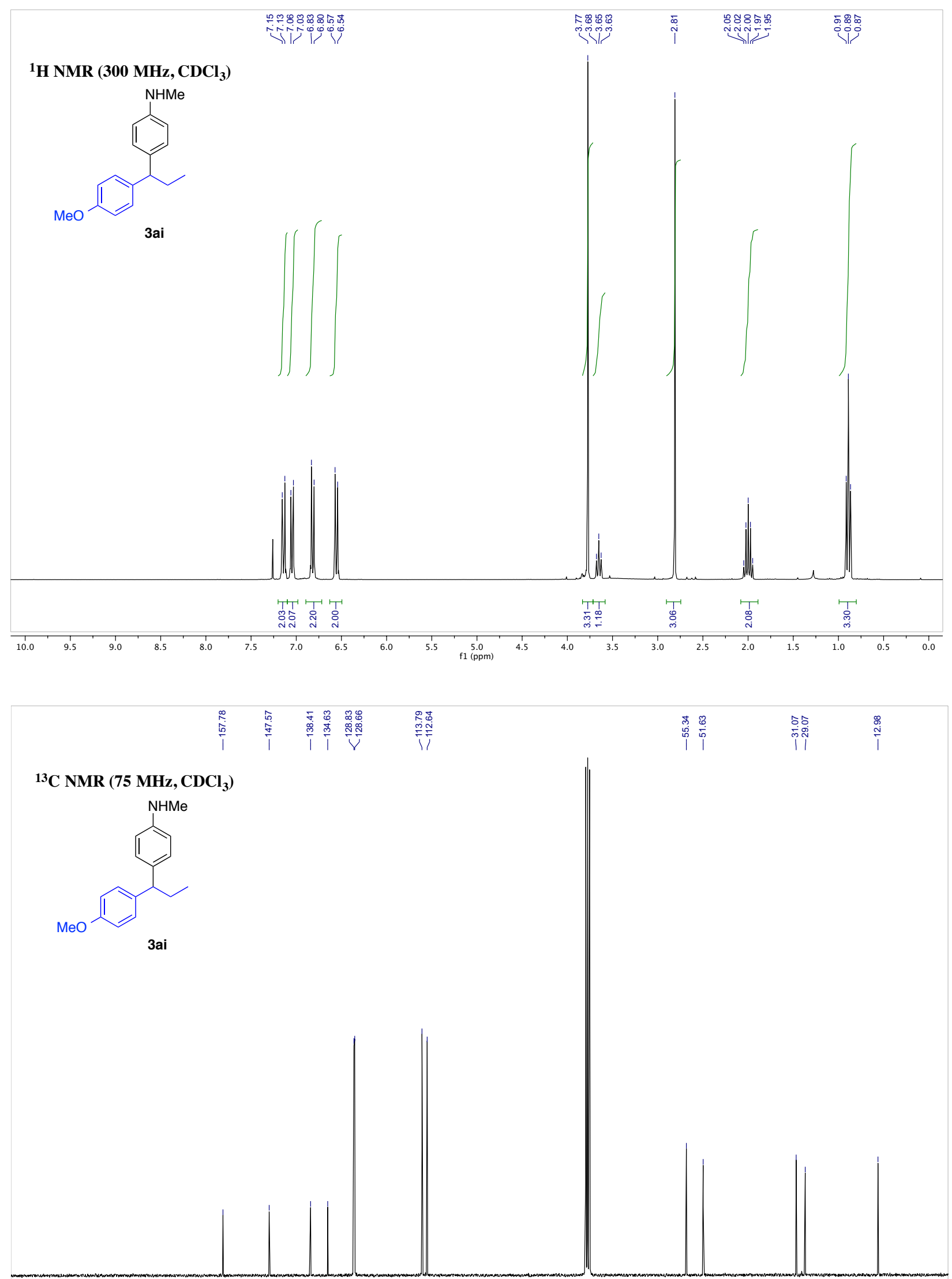

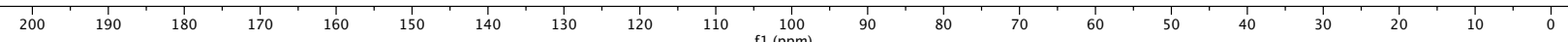



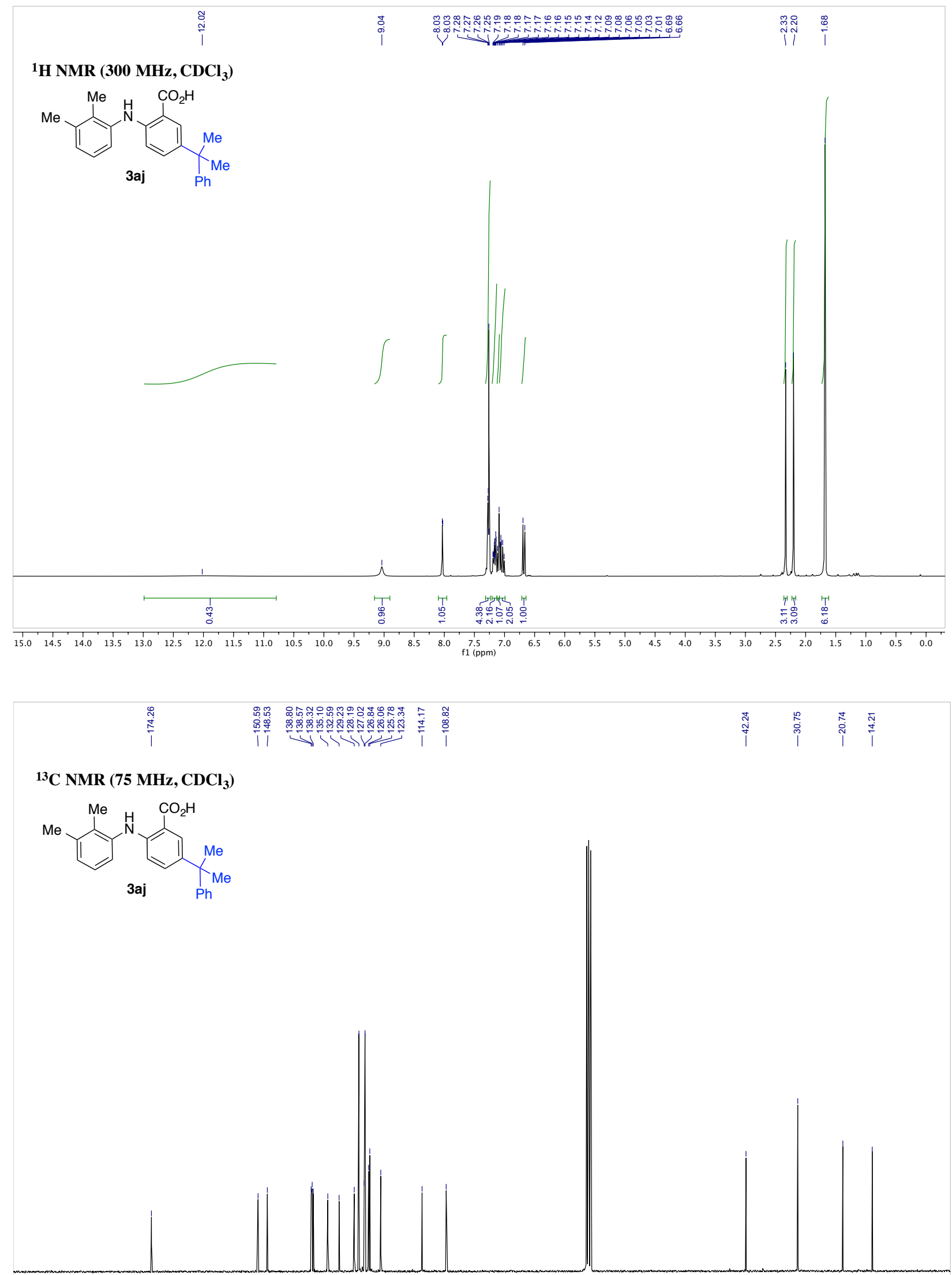

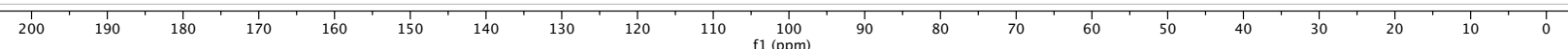




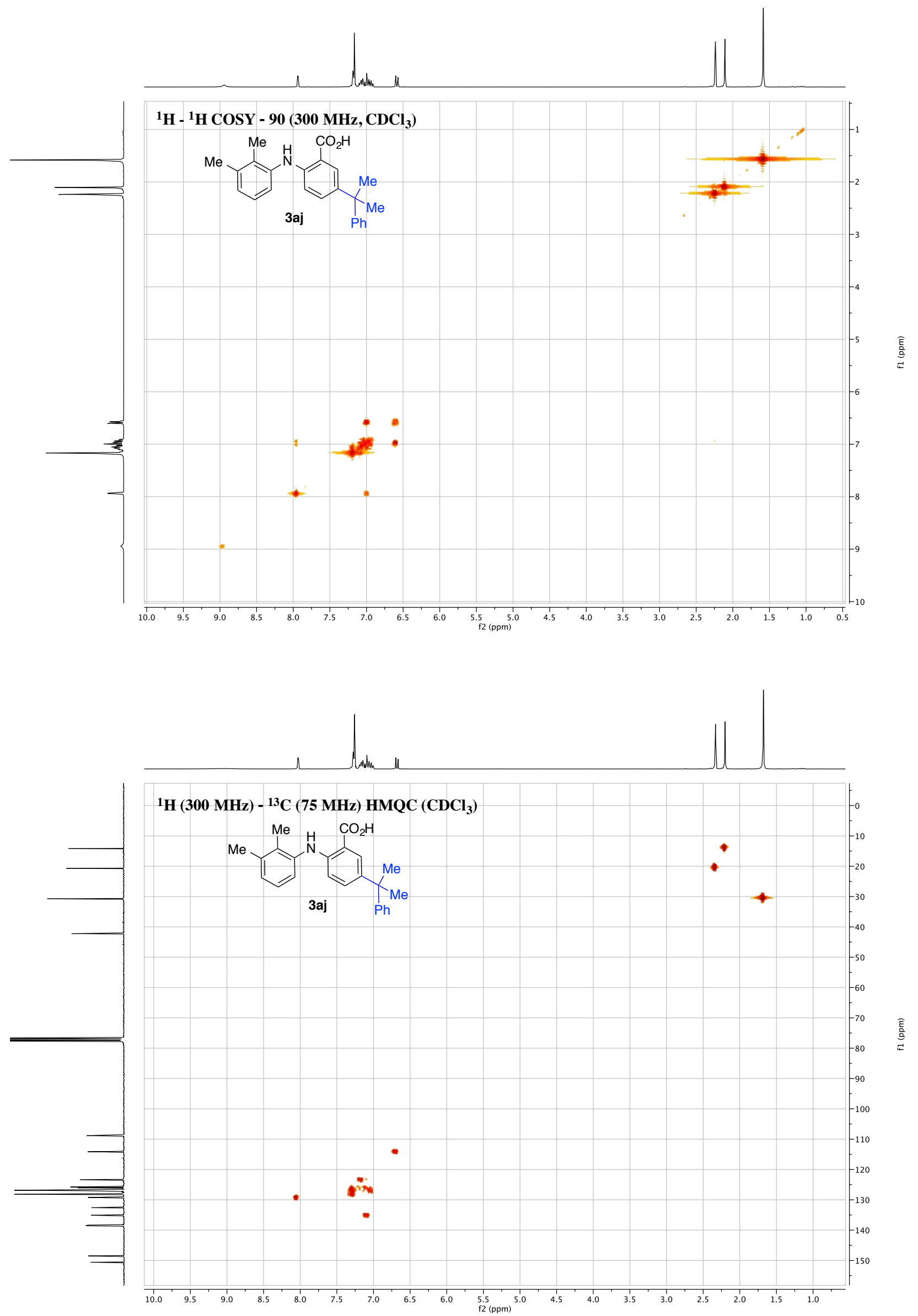



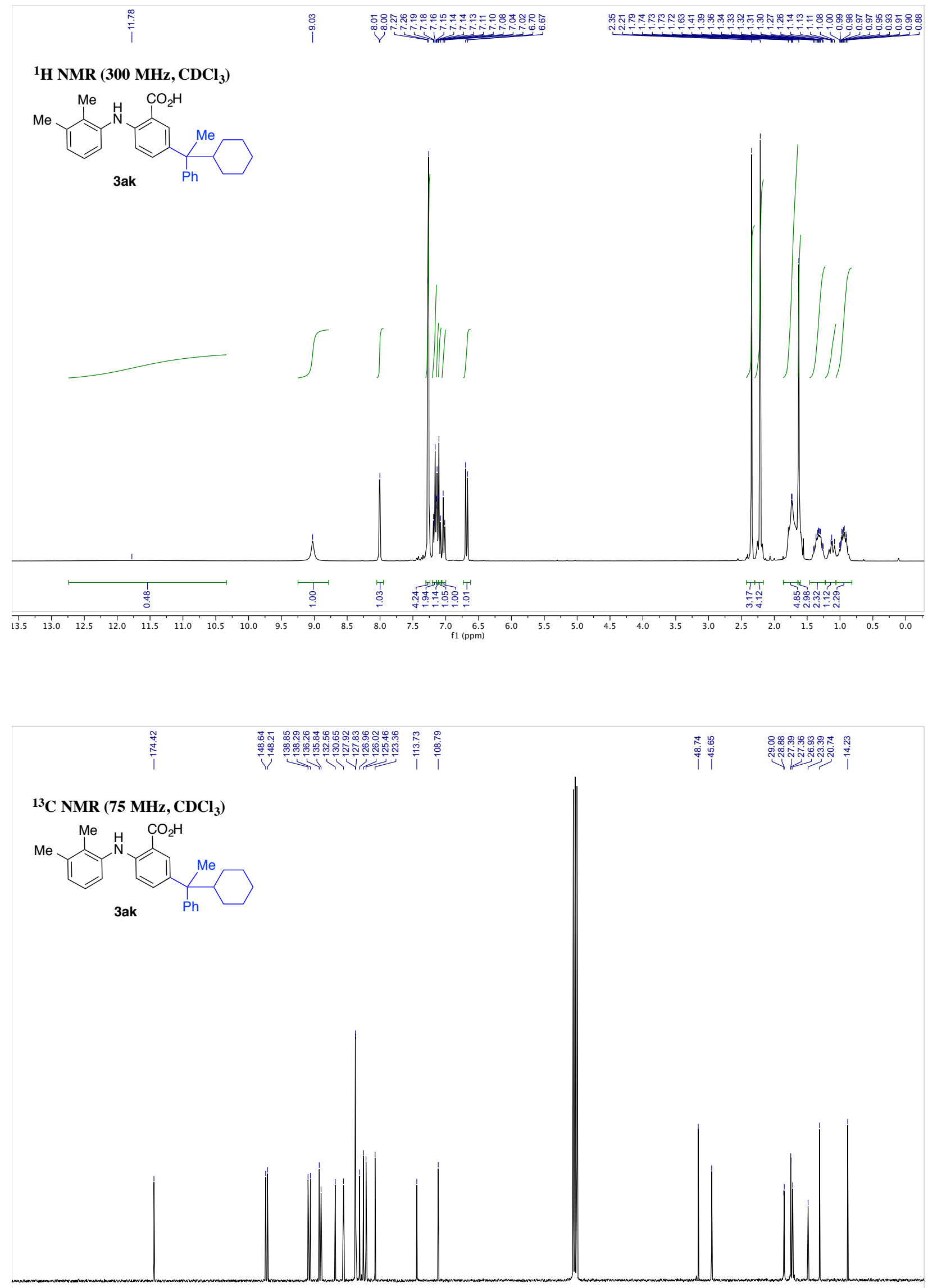

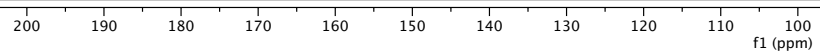



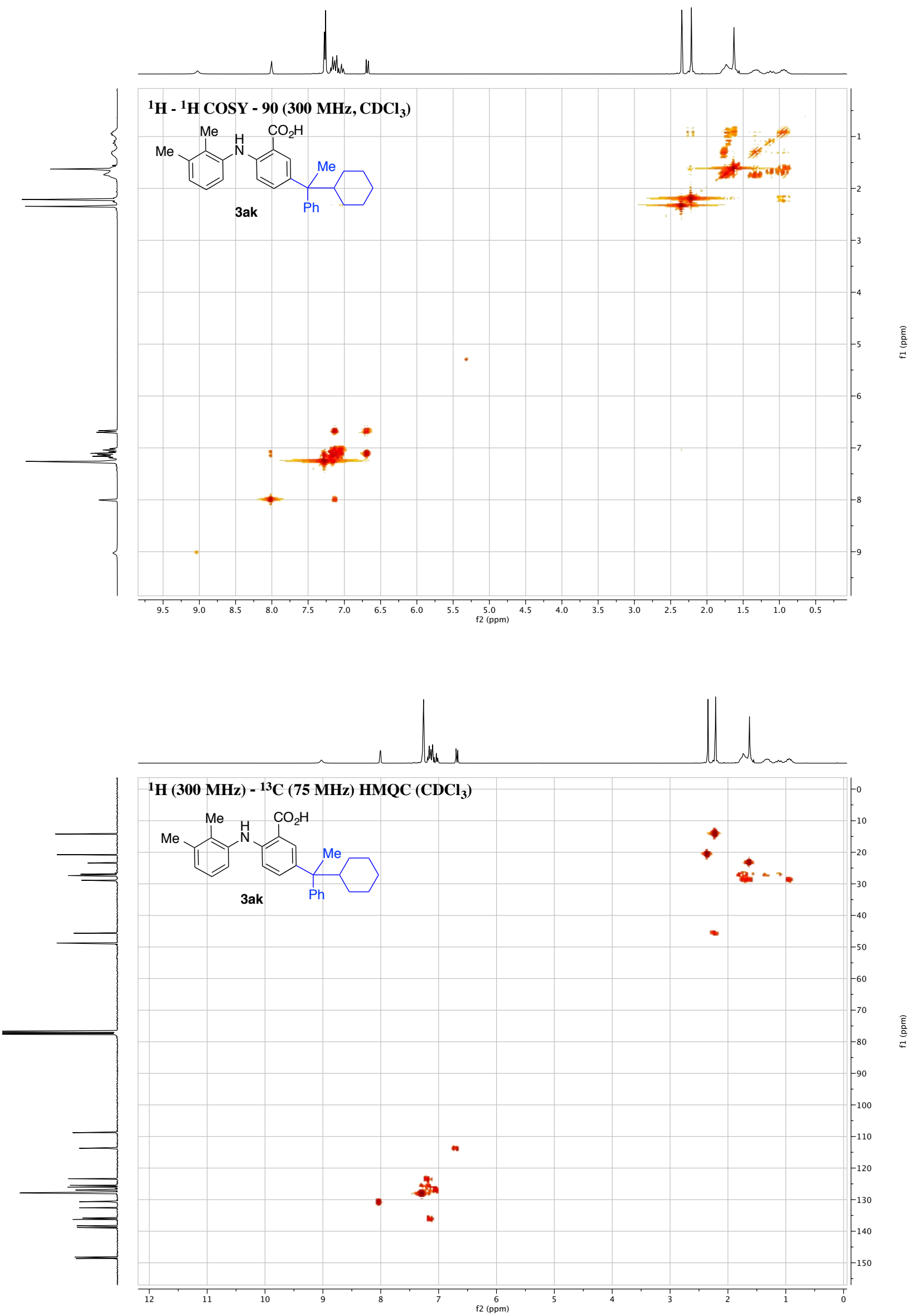


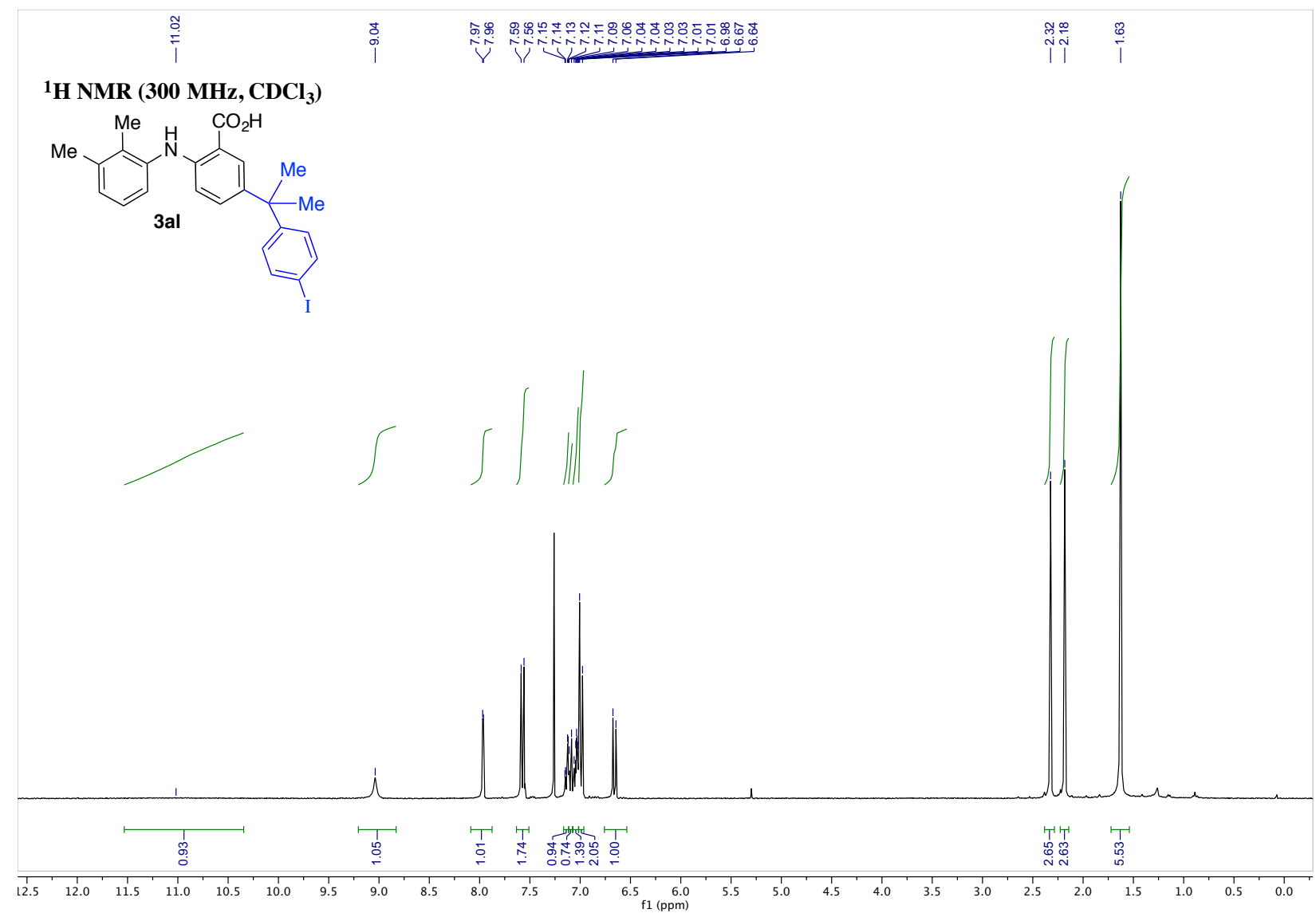

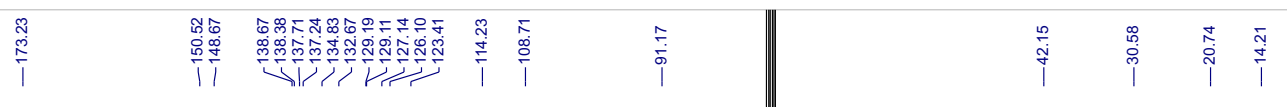

${ }^{13} \mathrm{C}$ NMR (75 MHz, $\mathrm{CDCl}_{3}$ )
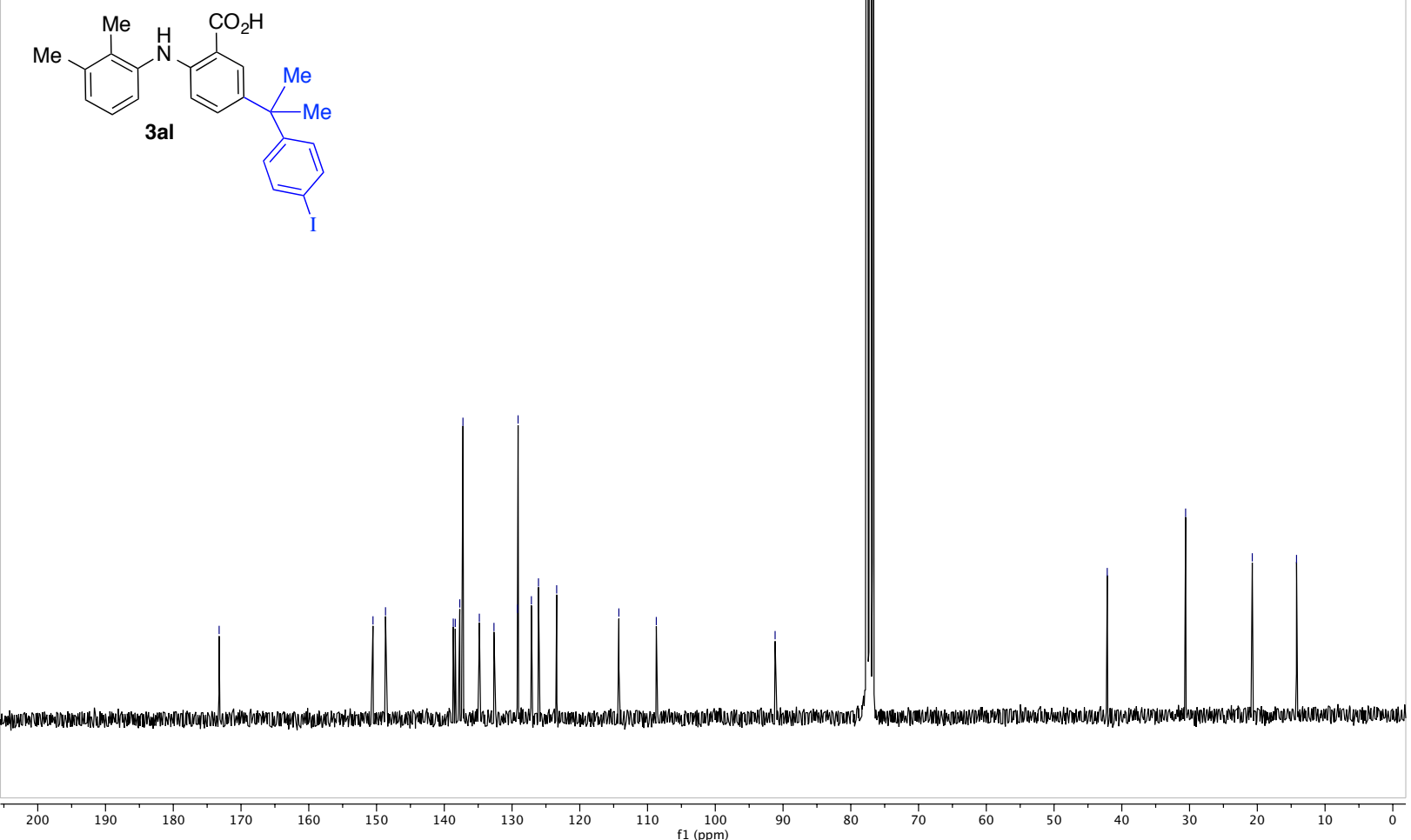
1 Lanl
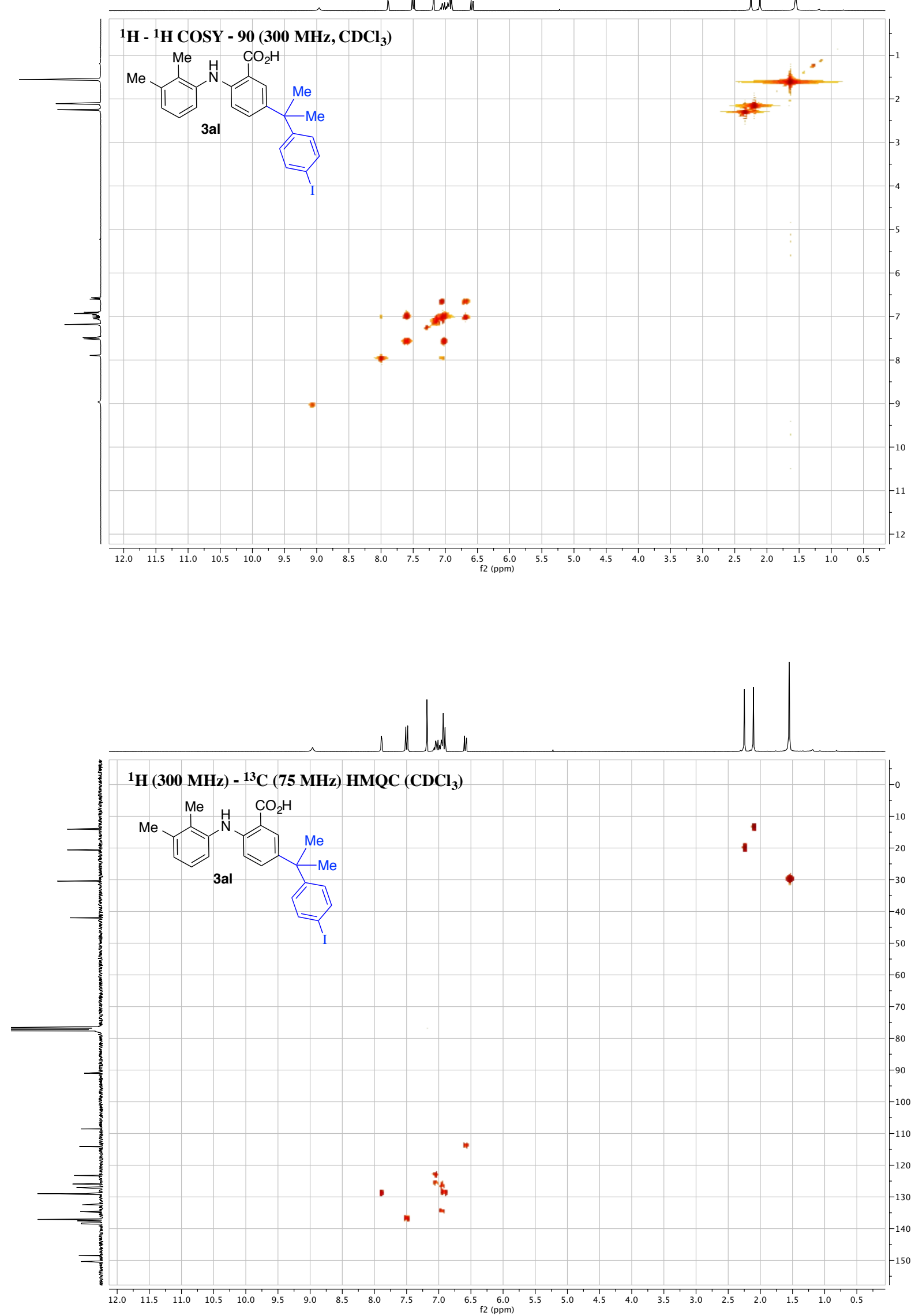
S87
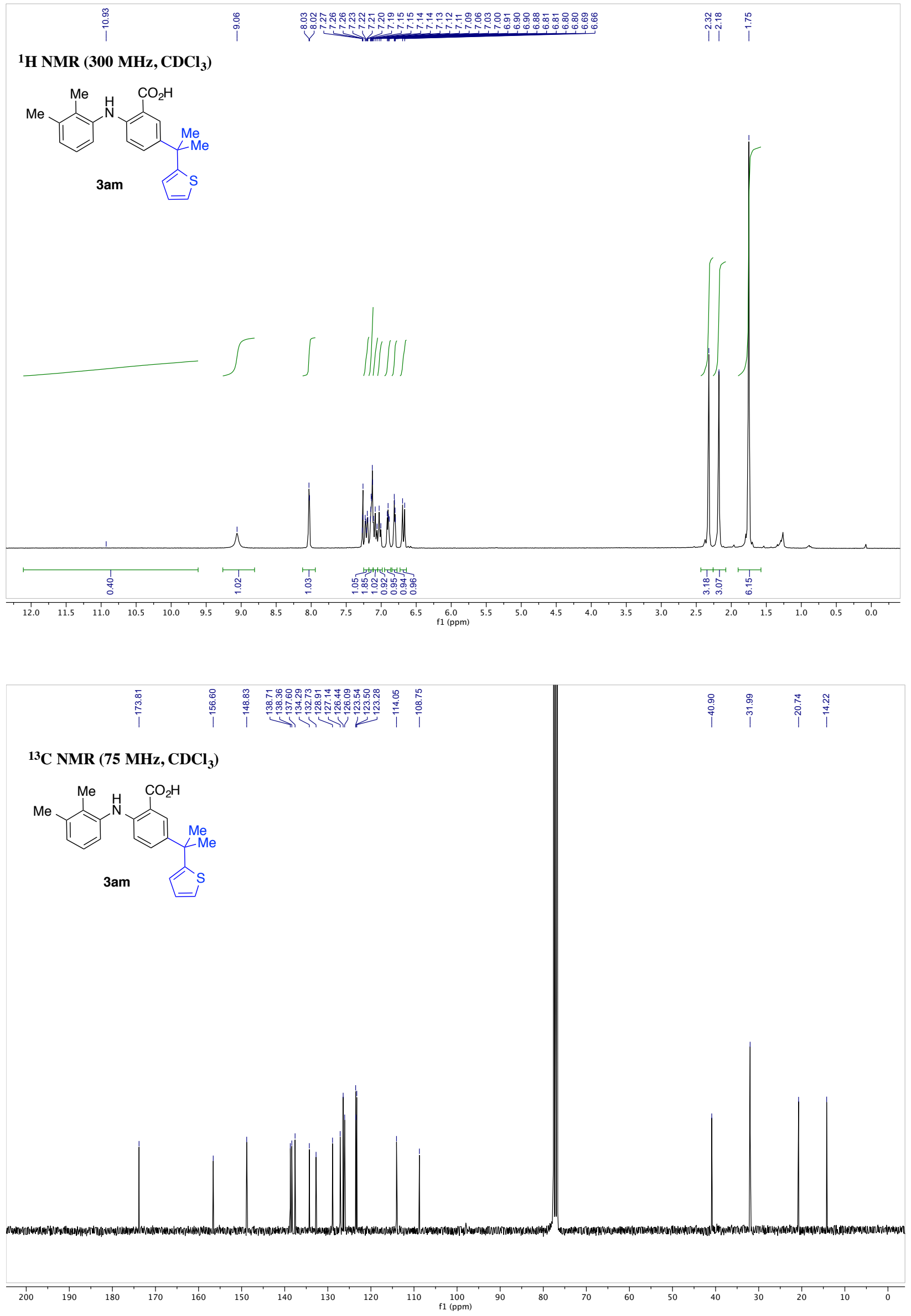

S87 
S88
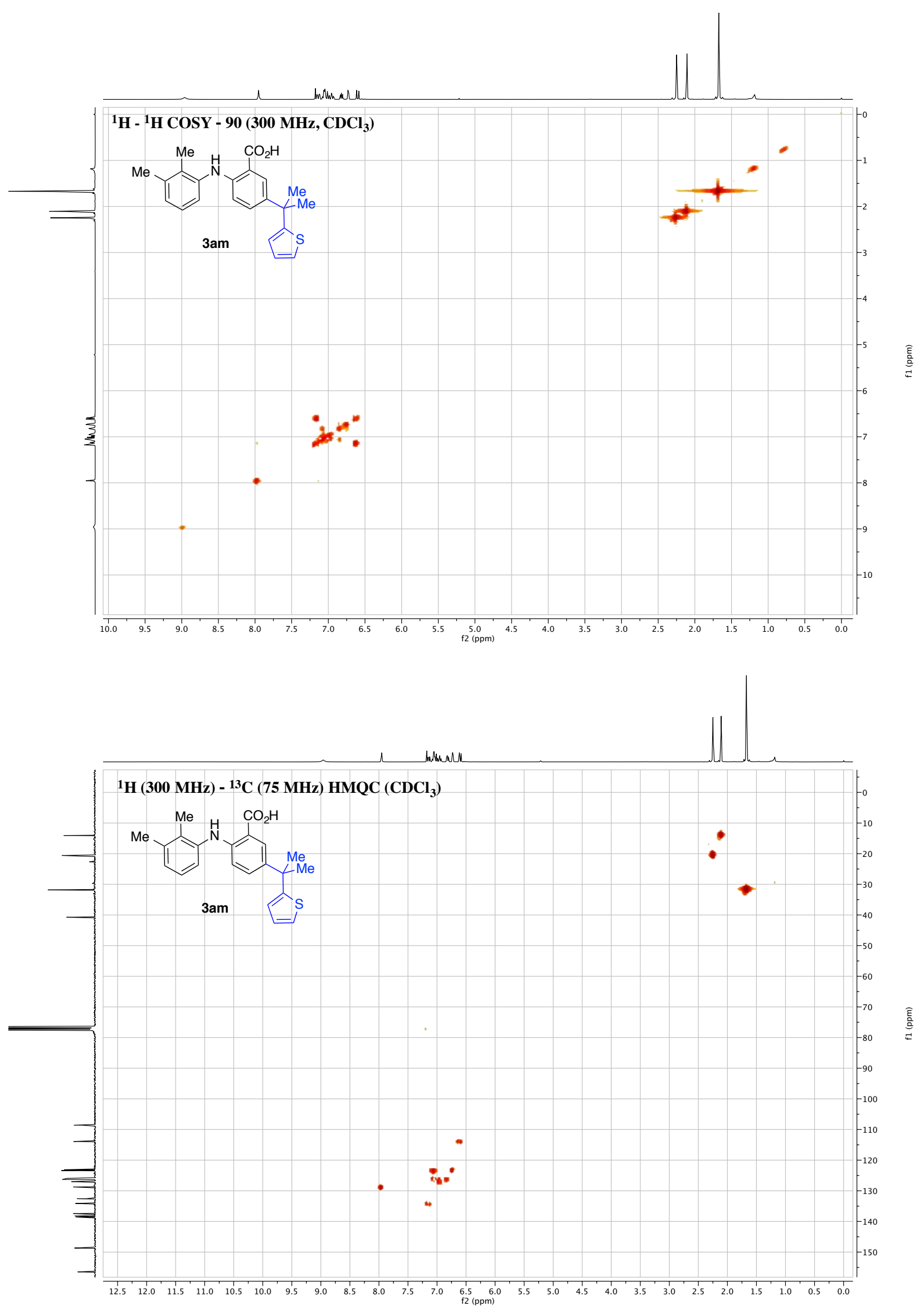

S88 

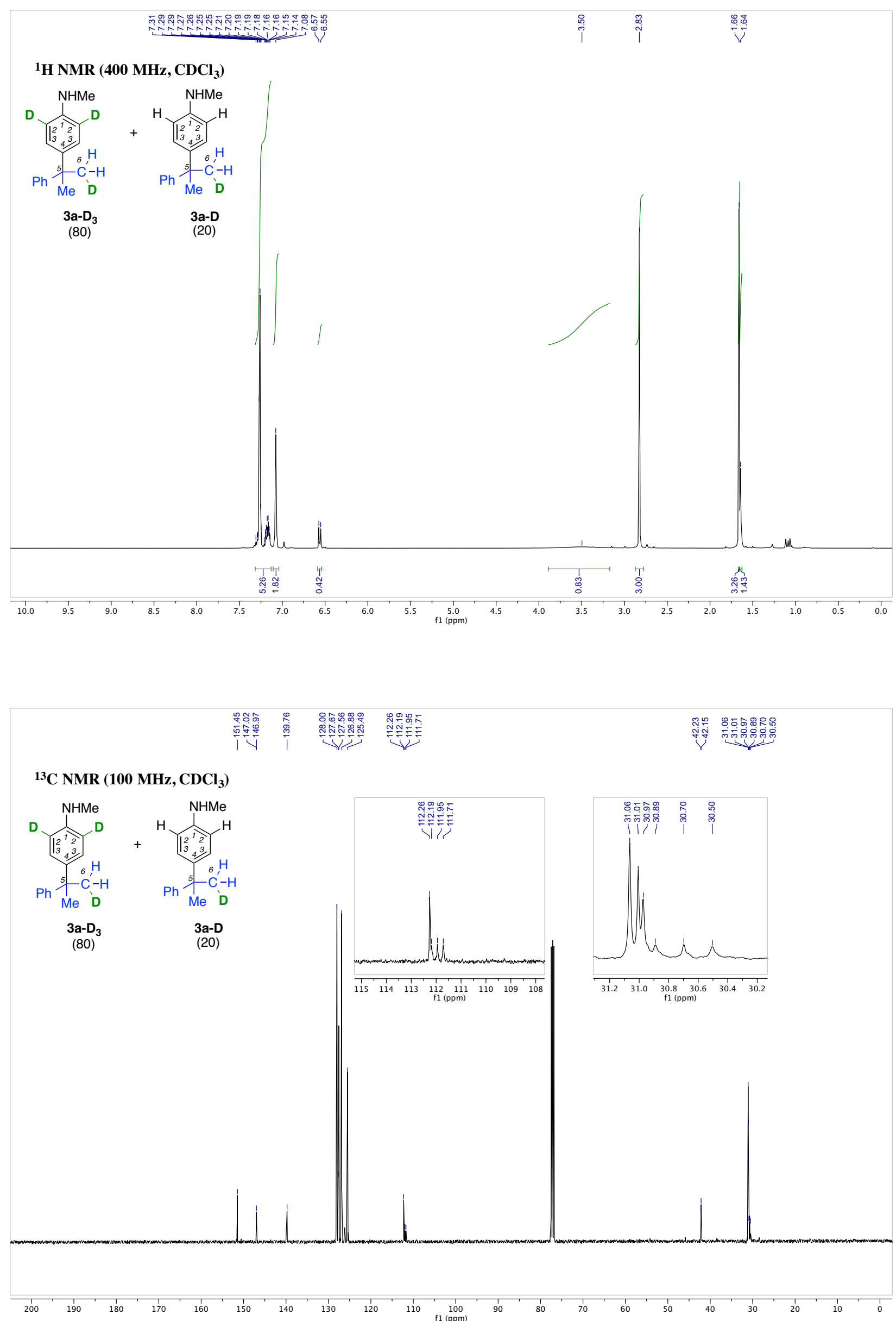

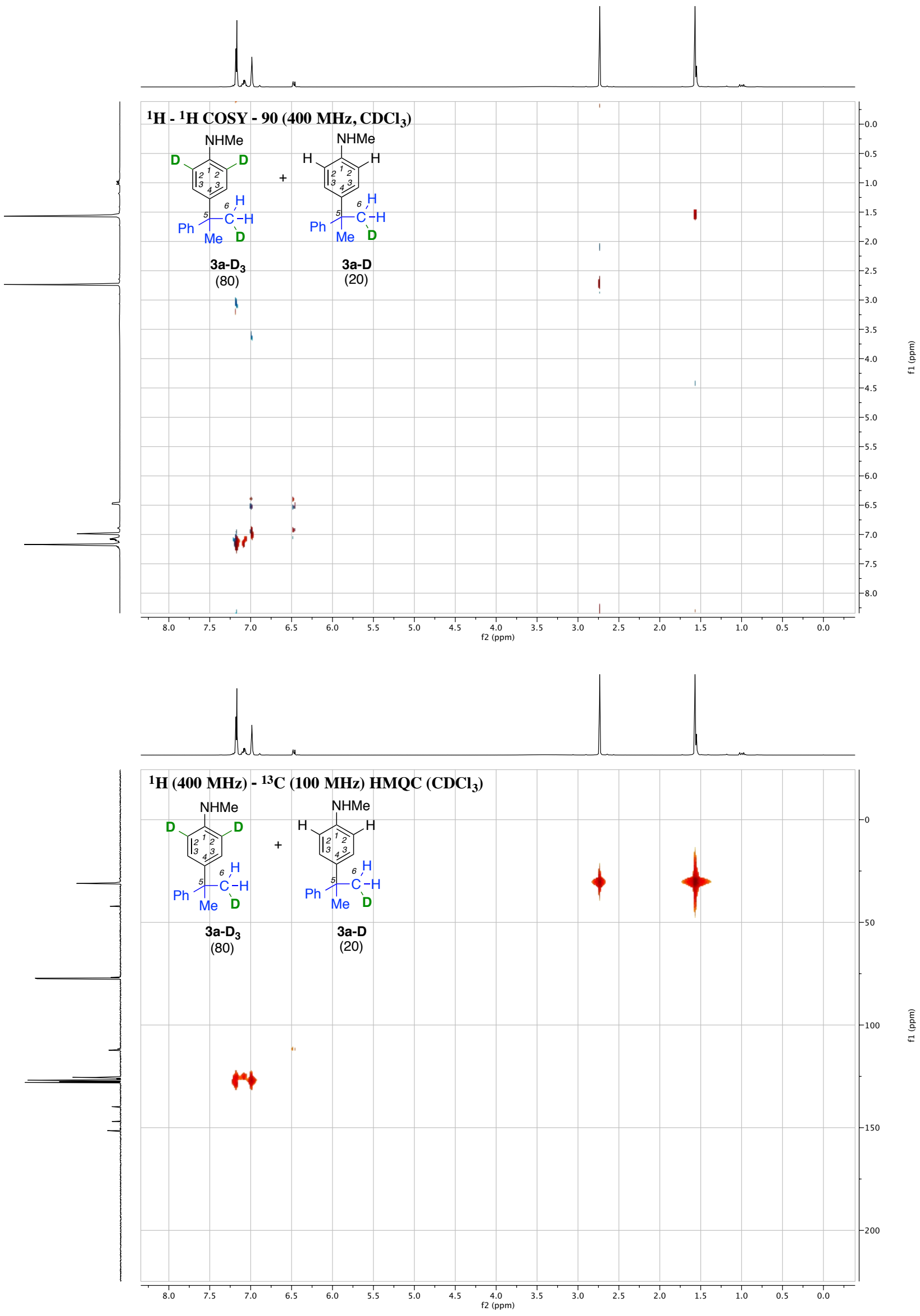


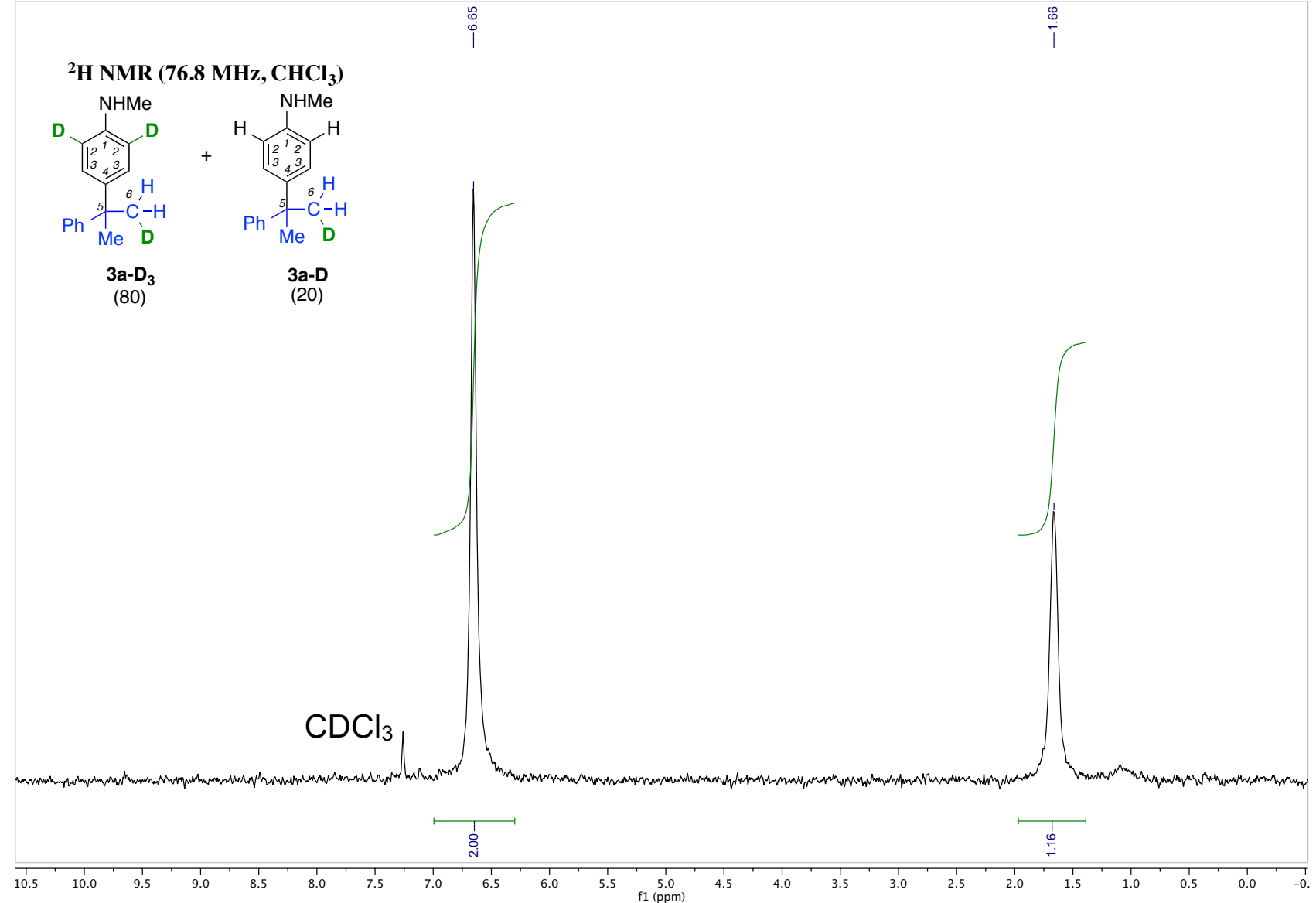



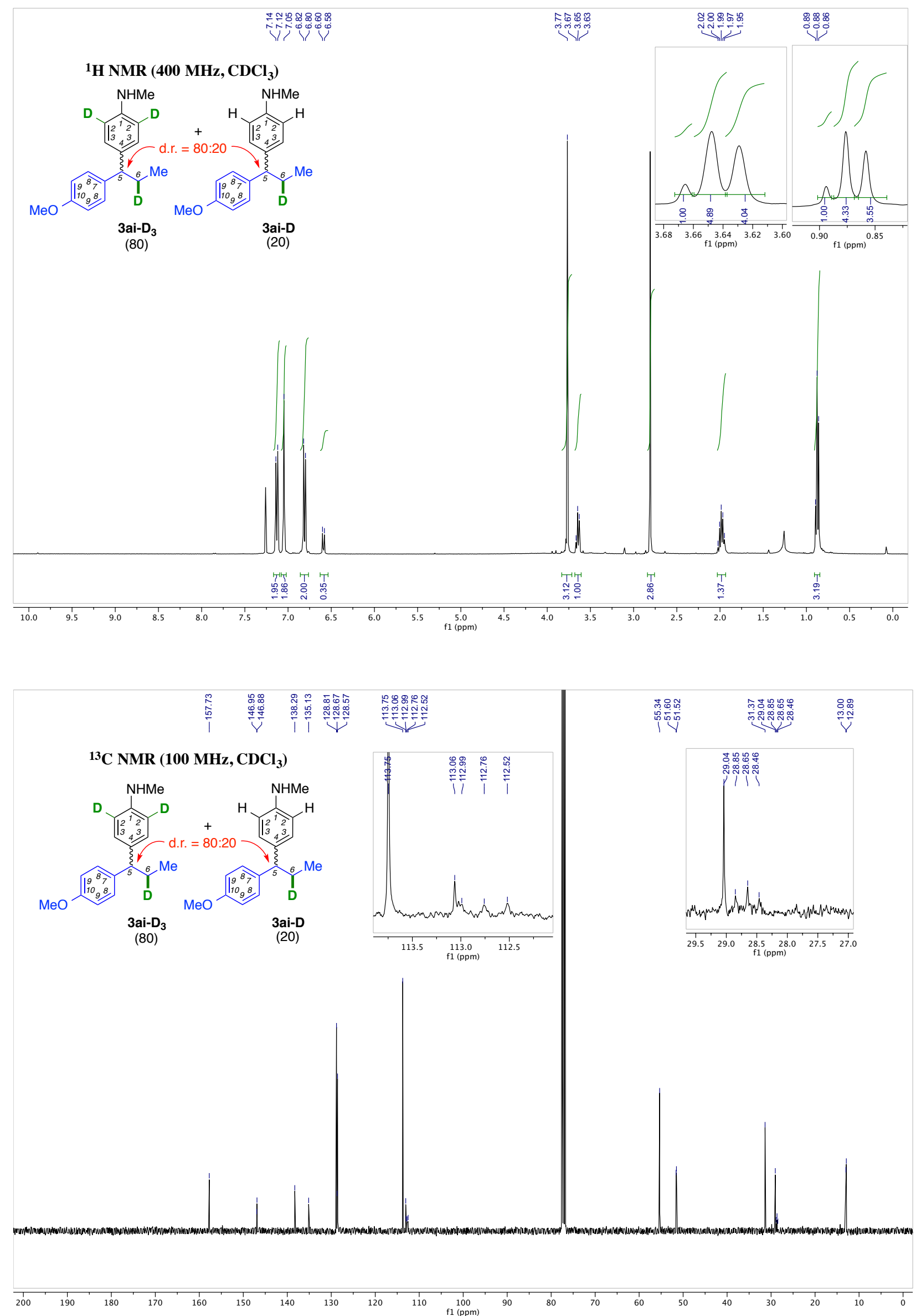
S93
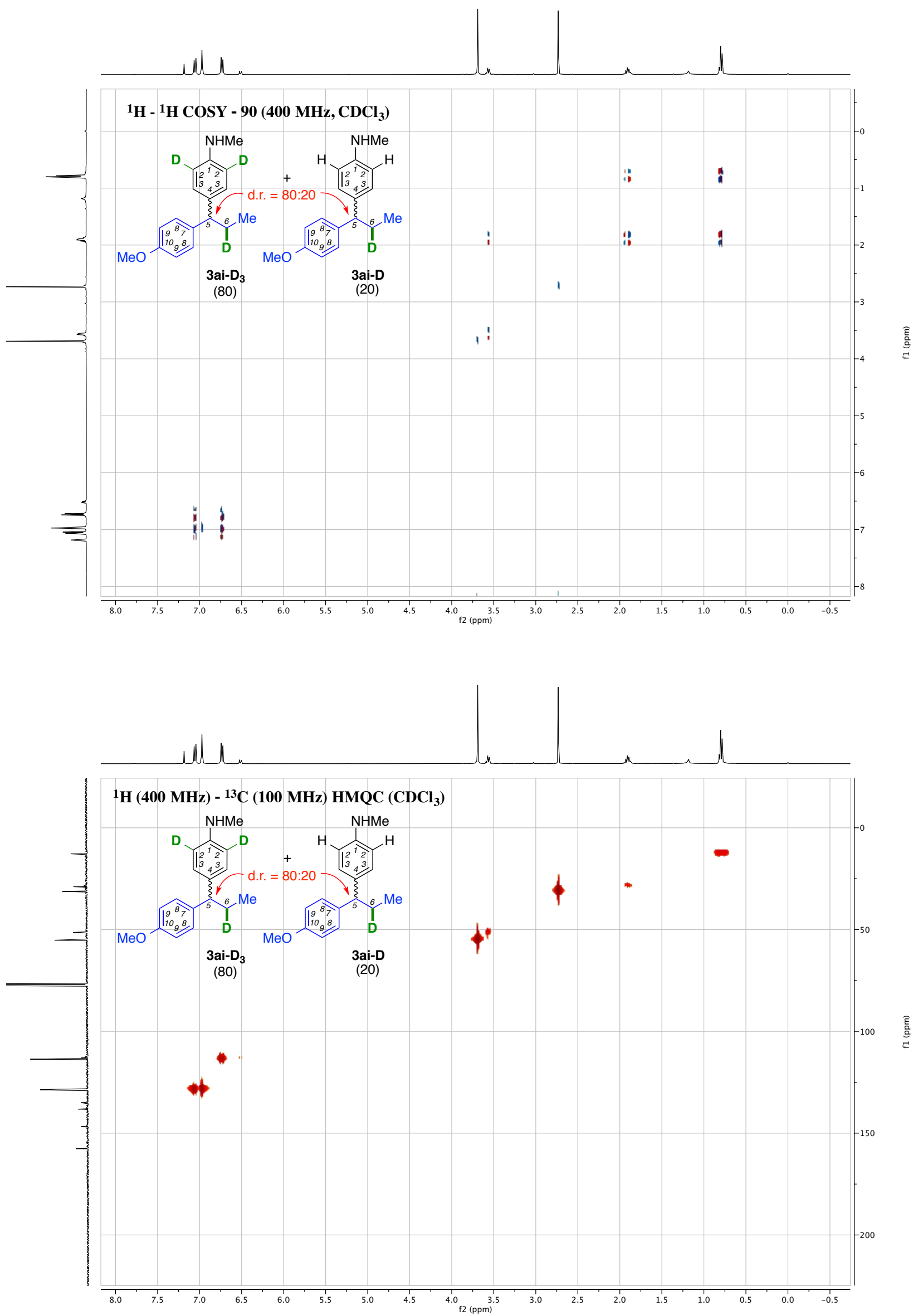

S93 
S94

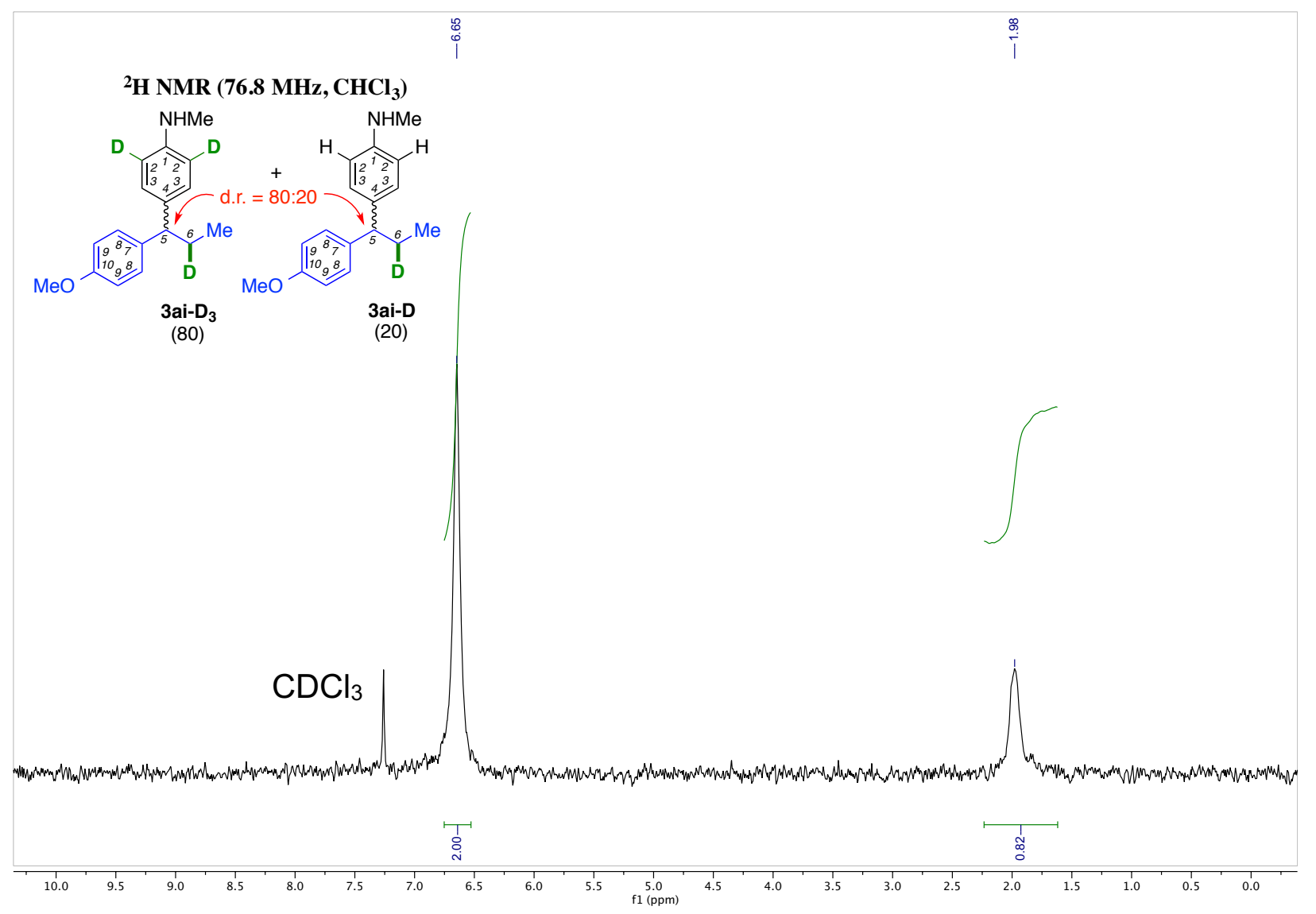

S94 

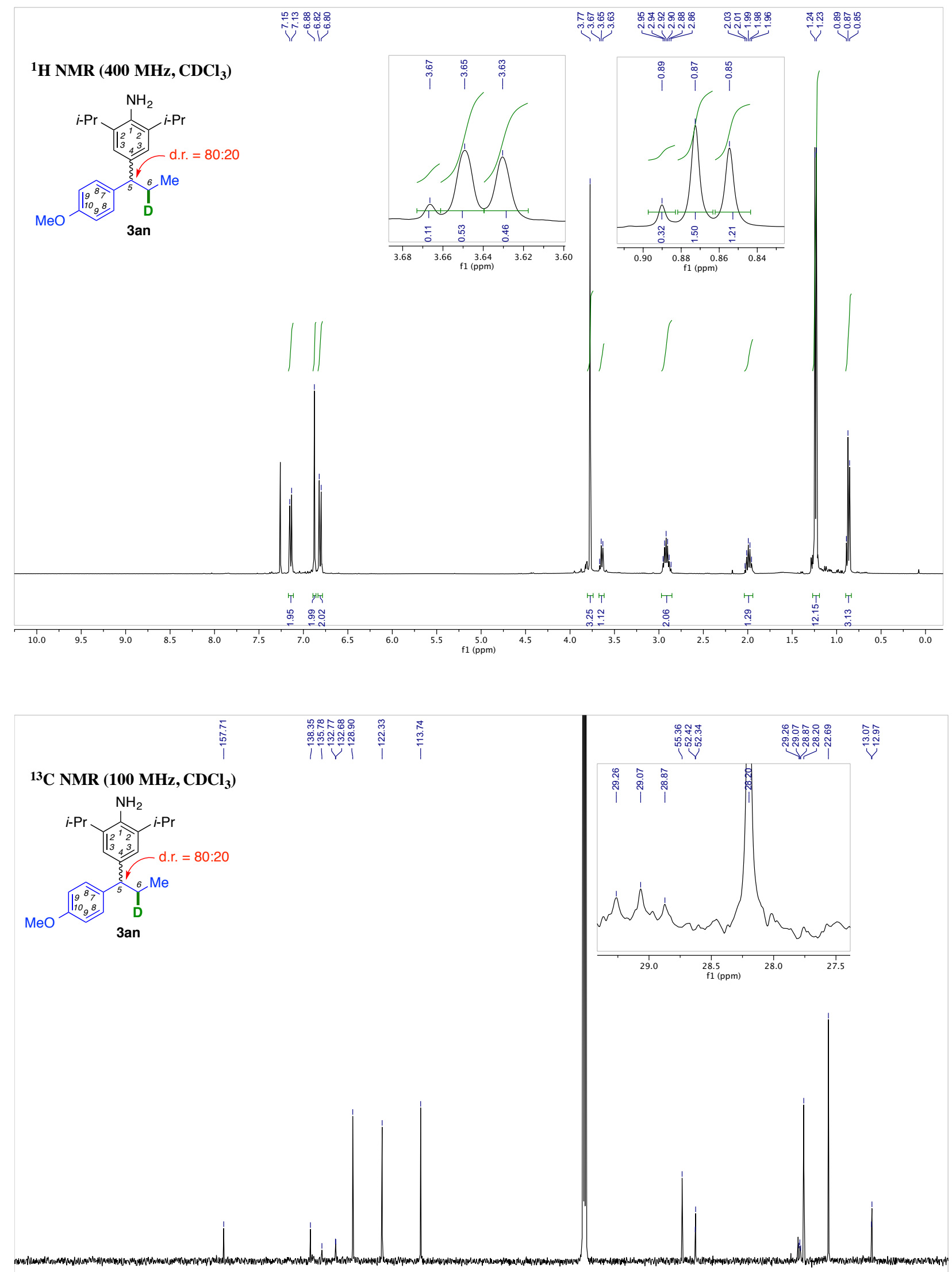

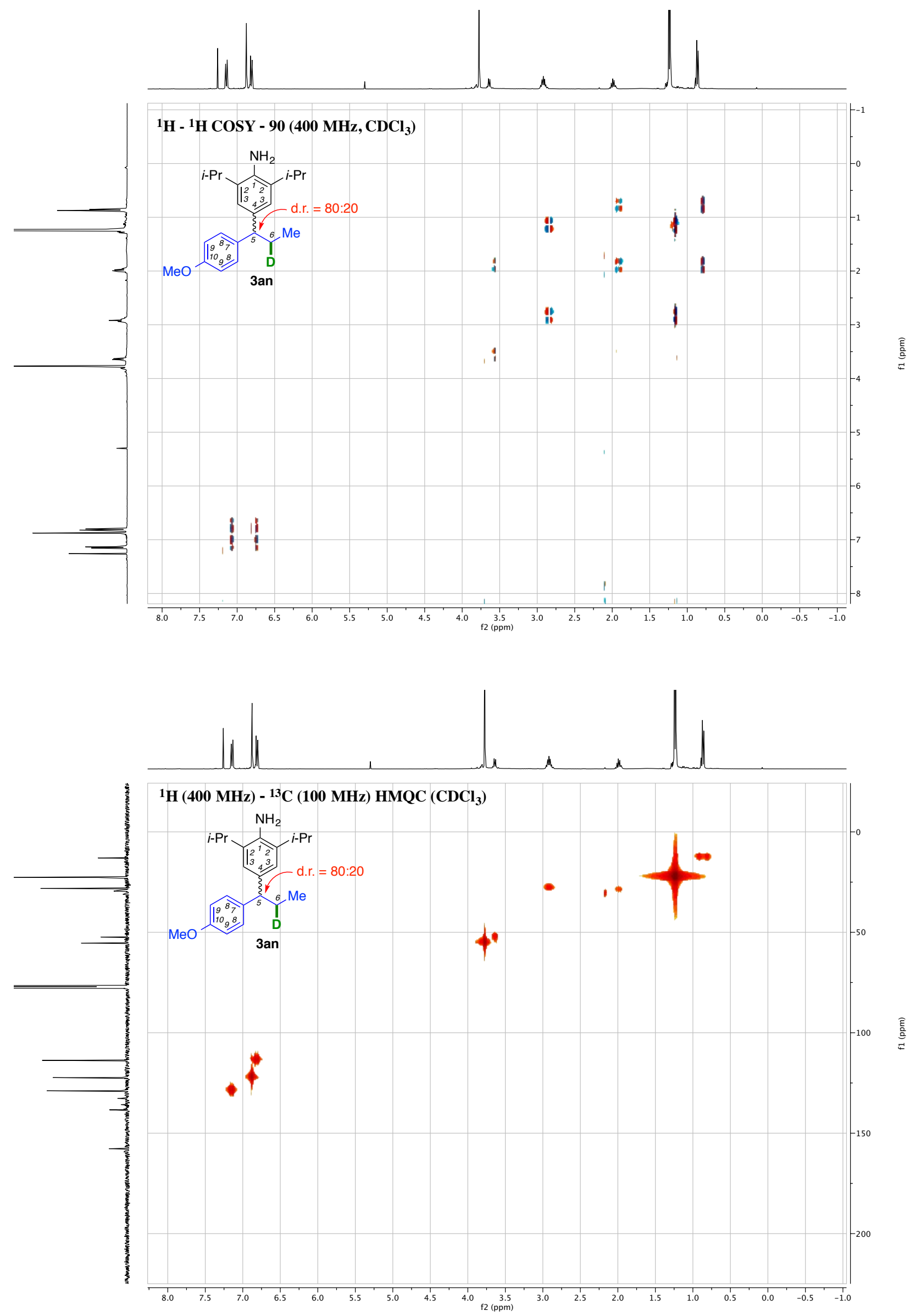
${ }^{2} \mathrm{H}$ NMR (76.8 $\left.\mathrm{MHz}, \mathrm{CHCl}_{3}\right)$
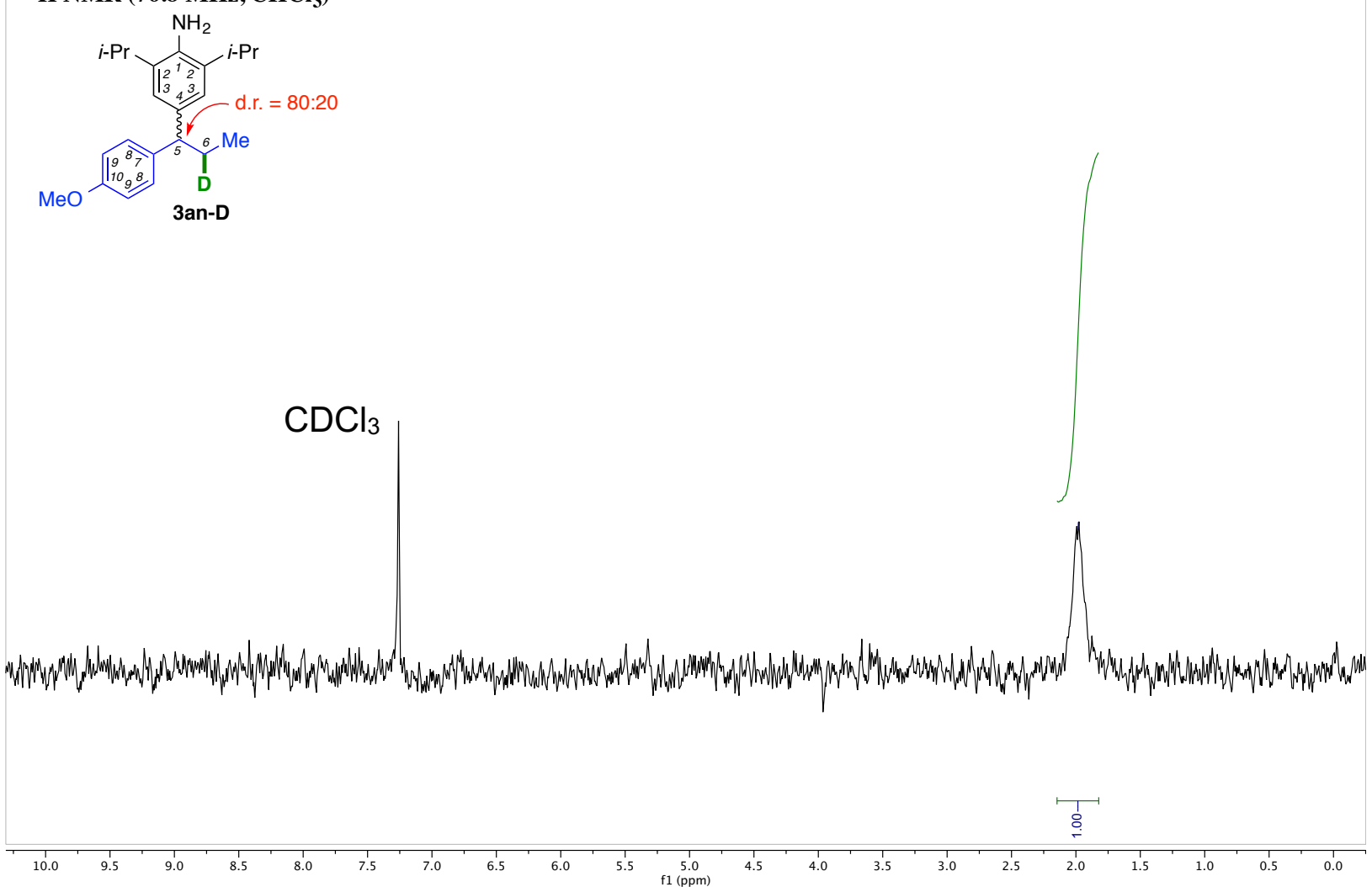

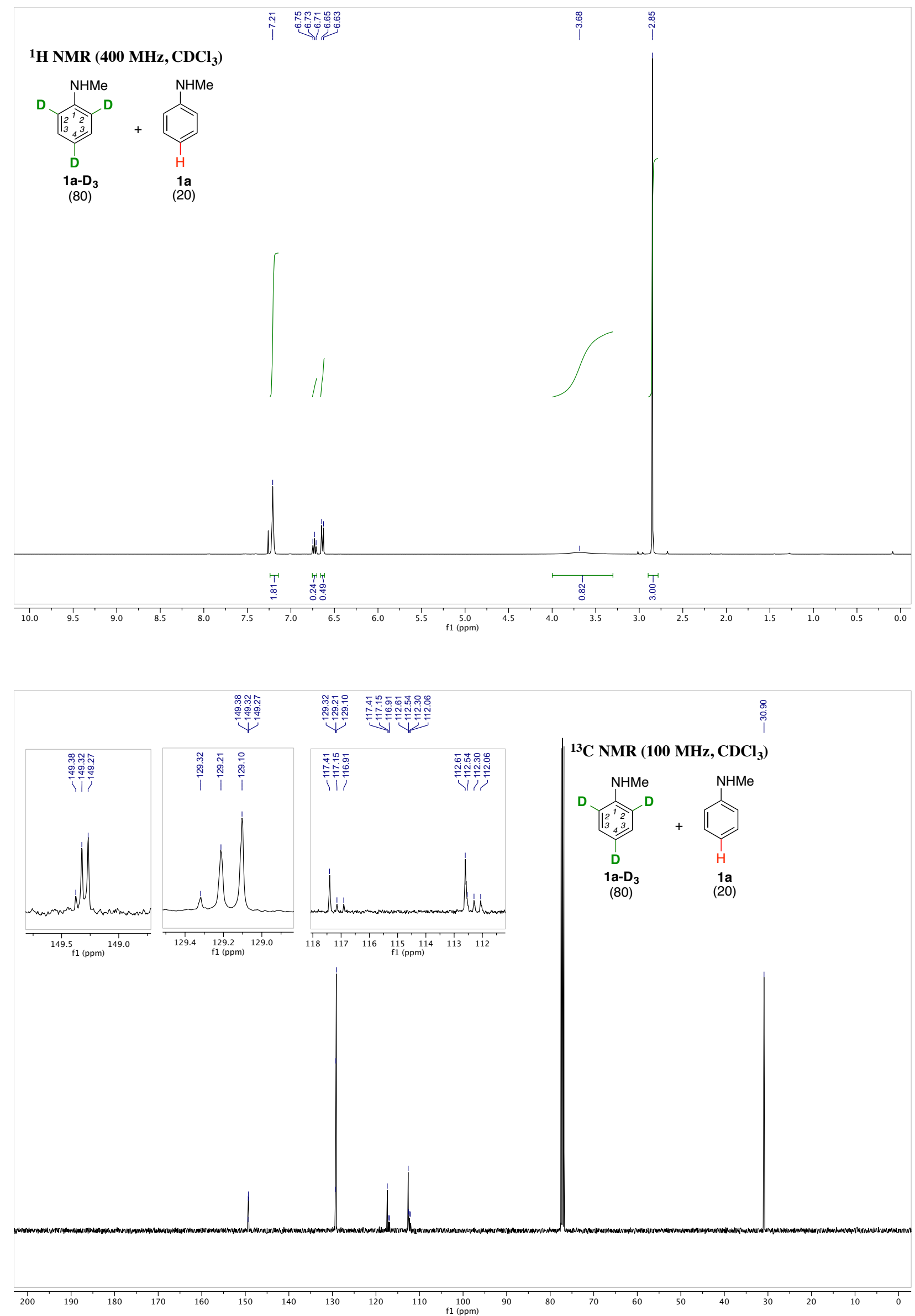
S99

com

${ }^{2} \mathrm{H}$ NMR (76.8 $\mathrm{MHz}, \mathrm{CHCl}_{3}$ )

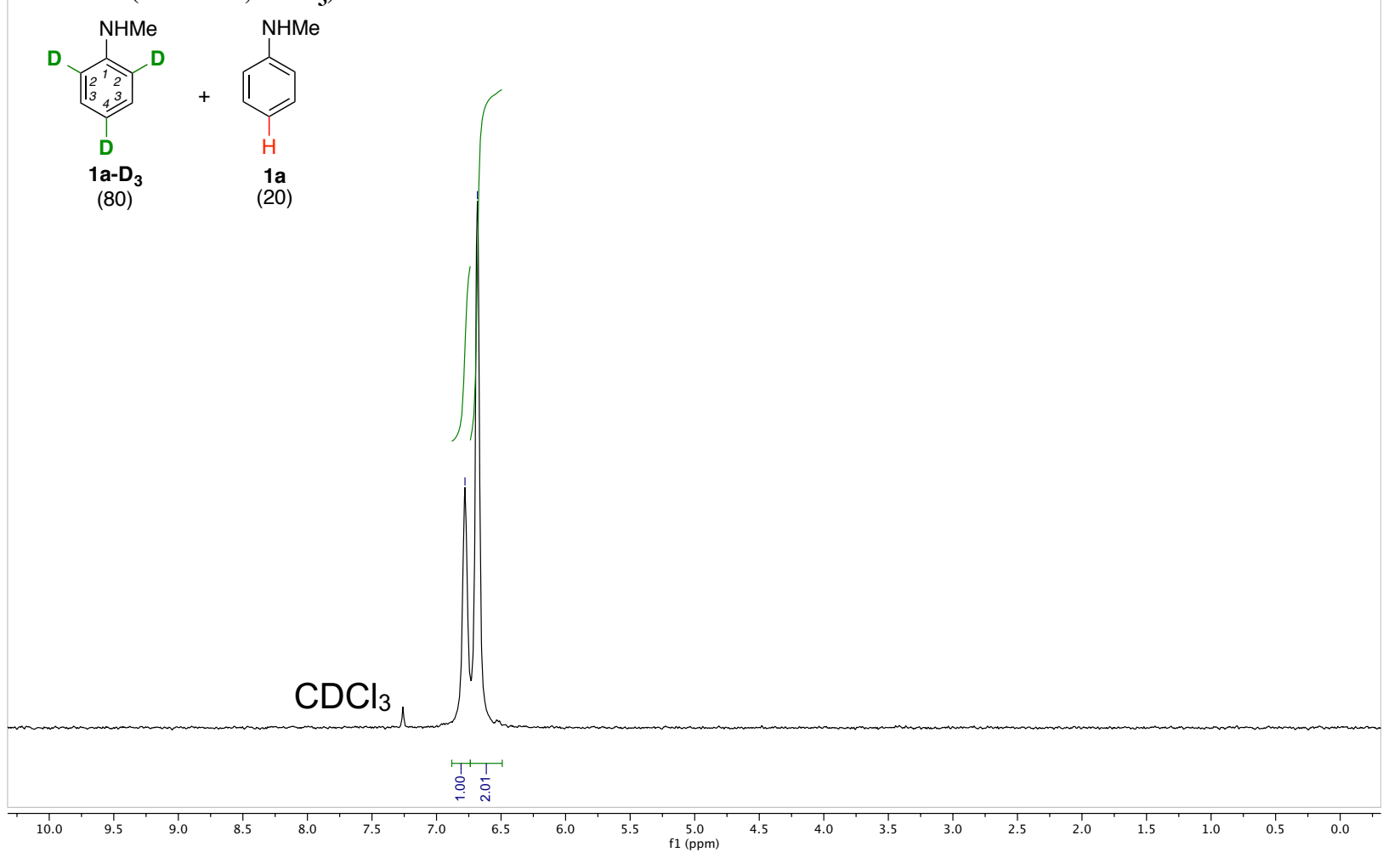

S99 